\title{
Planning Level Decision Support for the Selection of Robust Configurations of Airport Passenger Buildings
}

by

\author{
Tom Svrcek \\ Bachelor of Science in Industrial Engineering \\ University of Illinois at Urbana-Champaign \\ January 1988 \\ Certificate of Post Graduate Study in Management Science \\ Cambridge University, England \\ June 1989 \\ Master of Science in Operations Research \\ Massachusetts Institute of Technology \\ May 1991
}

Submitted to the Department of Civil and Environmental Engineering in Partial Fulfillment of the Requirements for the Degree of Doctor of Philosophy

Transportation

at the

Massachusetts Institute of Technology

May 1994

() Tom Svrcek 1994

The author hereby grants to MIT permission to reproduce and to distribute publicly copies of this thesis document in whole or in part.

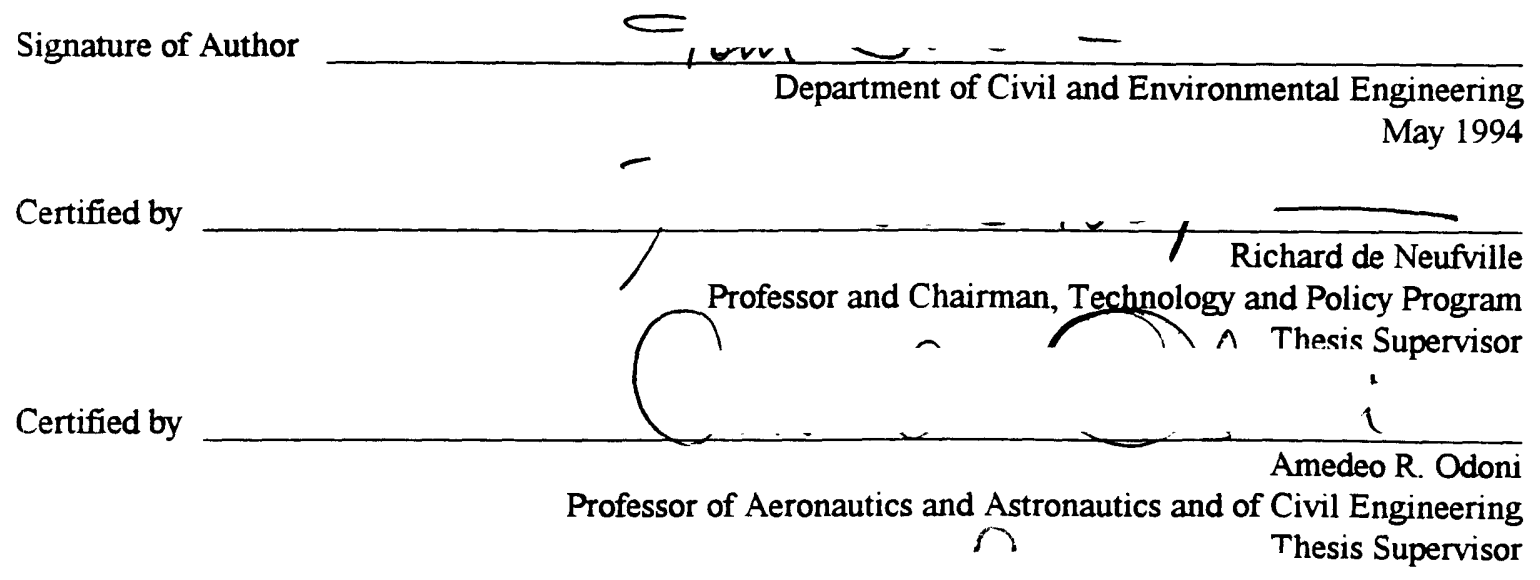


Planning Level Decision Support for the Selection of

Robust Configurations of Airport Passenger Buildings

\author{
by Tom Svrcek \\ Submitted to the Department of Civil and Environmental Engineering \\ on May 6, 1994 in Partial Fulfillment of \\ the Requirements for the Degree of \\ Doctor of Philosophy in Transportation
}

\begin{abstract}
Prevalent in the current practice of airport design is the view of the airport as a "terminal", or beginning and ending point only of a traveler's journey. Such a perspective wrongly encourages the belief that airport performance can be measured with only a limited number of criteria over a narrow range of conditions. This dissertation adopts the view that performance should be considered in broad range, multiple criteria terms, in order to balance the (often conflicting) objectives of the different users of an airport's services.

To accomplish this goal, we develop a series of prototype Passenger Building Configuration Evaluator (PBCE) tools, to help airport planners during the selection of an initial configuration concept. To study objectively the differences among dissimilar concepts, we identify a general airport configuration nomenclature and use it to represent several different concepts geometrically. From these characterizations, we obtain the absolute distances between any two points within the airport. Based on assumptions regarding the distribution of passengers, we then estimate the transition probabilities of traveling between any of these points. Using the PBCE tools and well-known results of geometrical probability, we show how we can estimate the potential performance of any given configuration and geometry.
\end{abstract}

Using a representation of data called a "performance profile", we demonstrate how planners can select an initial configuration based on robustness, or performance over a broad range. Specifically, we illustrate the selection process faced with uncertainty in such parameters as the level and type of transfer traffic, industry structure, and size. Our analyses suggest that configurations most appropriate for minimizing expected walking distances may not be the most appropriate for minimizing the expected taxi distances (and required number of turns) for aircraft.

We also address the issue of passenger congestion, both in pedestrian walkways and waiting areas. Included in our analysis is a discussion of the IATA level of service standards, and a survey of how current practitioners use these standards during the planning process. Finally, we show how simple, "back-of-the-envelope" type calculations can be used to obtain congestion estimates consistent with ones obtained from more detailed, data intensive methods.

Thesis Supervisors: $\quad$ Richard de Neufville

Title : Professor and Chairman, Technology and Policy Program

Amedeo R. Odoni

Title : Professor of Aeronautics and Astronautics and of Civil Engineering 
This body of work is lovingly dedicated to my mother, the most intelligent person I know ... 


\section{Acknowledgments}

I am very grateful to Professors Richard de Neufville and Amedeo Odoni for their patient and insightful supervision of this body of work. Special thanks also to Professors Robert "Bob" Simpson and Peter "Chief" Belobaba, who both provided exceptional ballast during the (more than) occasional encounter with "rough waters". More than simply serving on my committee, these men were my mentors and my friends throughout my tenure at MIT. And to Arnie Barnett, a very special friend, thank you for listening to my stories and sharing yours with me over lunches for the past fours years. I am also grateful to the Winston Churchill Foundation, the Office of Naval Research, the Federal Aviation Administration/TSC, and Sheffield Associates for providing me with much needed financial assistance.

I must also thank my family for their emotional and financial support throughout my entire college "career". When the rest of the world thought I was crazy for giving up the best years of my life to the stress and aggravation of graduate school, my family provided me with enough of both to make grad school life seem like a vacation. Anyone who knows my family knows that this is, of course, a heinous lie. Without their constant, often overwhelming love, I would never have been able to realize my dream. Mom, thanks for having me, and for nurturing within me your extraordinary intellectual curiosity. And if you hadn't saved my life, Linda, both when I was three and so many times since, none of who I am would have ever been possible.

Thanks also to my two best friends. Steve (Bernardo, Feinstein), my best friend in the entire world, thanks for twelve years (plus or minus a couple in dispute) of sharing everything from Matt's cookies and chocolate milk to answers on our computer science final. Although I may never forgive the kid in the green jacket, I will always cherish your companionship, your trust, and the five hundred bucks I still owe you. And to Kate, my new best friend, thank you for asking me out first and putting up with me for the past two years. You have been my companion and my strength. I promise never to apply to Law School or Med School.

Next, I salute a cast of true characters. To Wizzie, (Ms. Modest), who's life toil it is to make people feel insecure (silly hats, pencils, and Jennifer jokes included) but also loved. To Dave Kempka, whose prison sentence lasted just long enough for me to make a brilliant friend. To Vince Rosenthal, for asking me to stand at his wedding and helping to make storytelling history. To Joe Kraft, thank you for showing me your Chicago and for at least considering the name "Buck" for your child. Thank you also,

"Captain" Puco -- keep studying, and keep dodging. Kels, thanks for the constant encouragement, making me watch the "Drake tapes", and sharing most of our Saturday nights studying. And to all the other Kims and most of the Susans, a special thanks. 
I am also deeply indebted to the best group of athletic partners a man could hope to have. Thank you Christopher, for showing me what it means to "Go Animal". Thank you, George, for being my lifting partner even as you were trying to finish up. Thank you, Chipster, for always pushing me to be a better squash player than you are (another heinous lie). Thank you, Paris, for getting me thrown out of Dali's and Shay's, and losing your car at inopportune times. Thank you House, Wimp, Leslie, Swinburn, and the entire Churchill Men's II May Bumps (Blades) Crew -- I dedicate my oar in memory of Ken Macrae, the best coach I ever had. And thanks to Brandeis University for building international-sized squash courts (the real reason Graham and Wick play with me), and for establishing the FOBA program.

Thanks also to a very special group of people who went out of their way to help me when I most needed it. Without their guidance, patience, and time, I would still be trying to register for my first semester of classes. So, thank you Laura and Michele (you were there when it all began), Lisa, Paula, Clare, Cynthia, Jessie, and of course, my new friend, Astrid. You are all shining stars in my book. Also, I am grateful to the companionship I received from the gang at the Flight Transportation Lab: to Ray "Legs" Ausrotas, Biz Williamson, Ted Botimer, Catherine Bohutinsky, Chung Mak, David Cuthbert, Seth, and Michael, thanks for the memories; and to the folks at the OR Center : Rags, you were a hell of a deskmate, Mitchell, next to you, nobody could feel insecure, Rob, thanks for the last minute data, and Zhijong, thanks for being yOu.

A great big HEX thank you to the students of H-Entry, who have had to put up with a sometimes non-existent, always trouble-making graduate tutor for the past four years. Although I am not supposed to play favorites, I did, and you guys know who you are. Finally, thank you to Tom Army at Rebecca's Cafe, the gang at the Coffee Connection and Karen at Il Dolce Momento for helping me with the "Java Jive", to Ray Charles for learning to play the piano, to Giuseppe Verdi for writing "La Traviata", and to the parents of Placido Domingo, for finding each other and having the greatest tenor the world has ever known ... 


\section{Contents}

1 Introduction $\quad 13$

1.1 Motivation $\ldots \ldots \ldots \ldots \ldots \ldots \ldots \ldots \ldots \ldots \ldots \ldots \ldots \ldots \ldots \ldots$

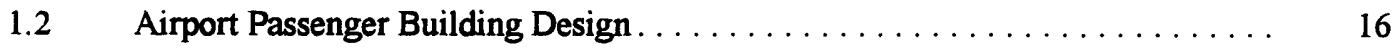

1.2.1 Single Forecast for the Future :

The Airport Building as a "Terminal" . . . . . . . . . . . . 17

1.2.2 Single (or Few) Performance Criteria $\ldots \ldots \ldots \ldots \ldots \ldots \ldots$

1.3 Robustness in the Design of Airport Passenger Buildings . . . . . . . . . . . 23

1.4 Broad Range, Multiple Criteria Performance . . . . . . . . . . . . . . . . . . . . 24

1.4.1 Broad Range Performance as a Design Objective . . . . . . . . . . . . 26

Multiple Dimensions of Performance for an
Airport Passenger Building $\ldots \ldots \ldots \ldots \ldots \ldots \ldots \ldots \ldots \ldots \ldots \ldots$

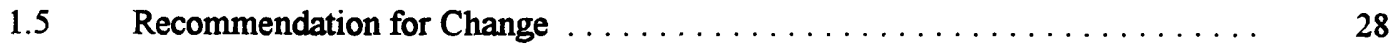

1.6 Problem Definition and Structure of Thesis $\ldots \ldots \ldots \ldots \ldots \ldots$

2 Review of Decision Support Methodologies 33

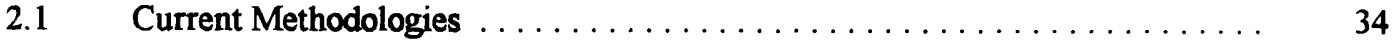

2.1.1 Step One : Selection of Initial Configuration and Geometry . . . . . . 35

2.1.2 Step Two : Detailed Layout of Floor Plan . . . . . . . . . . . . 37

2.1 .3 Filling the Gap . . . . . . . . . . . . . . . . . . . 39

$2.2 \quad$ Reference Manuals and Texts $\ldots \ldots \ldots \ldots \ldots \ldots \ldots \ldots \ldots \ldots \ldots$

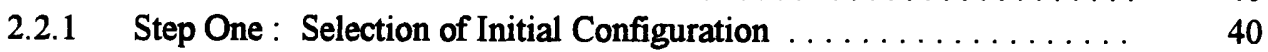

2.2.2 Step Two : Detailed Layout of Floor Plan . . . . . . . . . . . . . . . 43

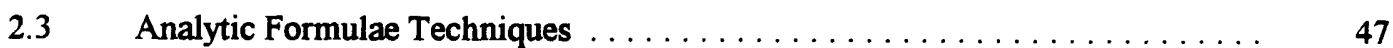

2.3.1 Passenger Walking Distances $\ldots \ldots \ldots \ldots \ldots \ldots \ldots \ldots \ldots \ldots$

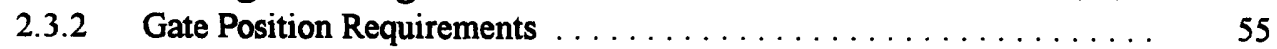

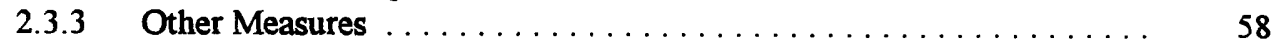

$2.4 \quad$ Simulation-Based Decision Support $\ldots \ldots \ldots \ldots \ldots \ldots \ldots \ldots \ldots \ldots \ldots \ldots \ldots$

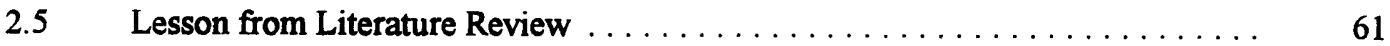

3 Airport Configuration Concepts $\quad 64$

3.1 The Gate Arrival Configuration $\ldots \ldots \ldots \ldots \ldots \ldots \ldots \ldots \ldots \ldots \ldots \ldots \ldots$

$3.2 \quad$ The Pier Configuration $\ldots \ldots \ldots \ldots \ldots \ldots \ldots \ldots \ldots \ldots \ldots \ldots \ldots$

3.3 The Satellite Configuration $\ldots \ldots \ldots \ldots \ldots \ldots \ldots \ldots \ldots \ldots \ldots \ldots$

3.4 The Transporter Configuration $\ldots \ldots \ldots \ldots \ldots \ldots \ldots \ldots \ldots \ldots \ldots$

3.5 The Midfield Configuration $\ldots \ldots \ldots \ldots \ldots \ldots \ldots \ldots \ldots \ldots \ldots$

$3.6 \quad$ Hybrid Configurations $\ldots \ldots \ldots \ldots \ldots \ldots \ldots \ldots \ldots \ldots \ldots$ 


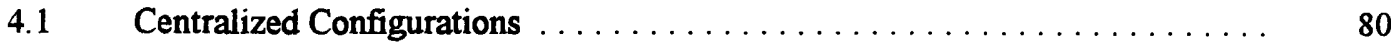

4.1.1 The Gate Arrival Concept $\ldots \ldots \ldots \ldots \ldots \ldots \ldots \ldots \ldots \ldots . \ldots \ldots$

4.1.2 The Parallel Pier Concept $\ldots \ldots \ldots \ldots \ldots \ldots \ldots \ldots \ldots \ldots \ldots . \ldots 1$

4.1.3 The Satellite Concept $\ldots \ldots \ldots \ldots \ldots \ldots \ldots \ldots \ldots \ldots \ldots . \ldots 2$

4.1.3.1 Circular Satellites ..................... 84

4.1.3.2 Rectangular and " $T$ "-Shaped Satellites ........... 86

4.1.4 The Midfield Concept $\ldots \ldots \ldots \ldots \ldots \ldots \ldots \ldots \ldots \ldots \ldots . \ldots \ldots$

4.1.4.1 Parallel Linear ..................... 88

4.1.4.2 Midfield " $\mathrm{X} " \ldots \ldots \ldots \ldots \ldots \ldots \ldots \ldots \ldots \ldots . \ldots$

$4.2 \quad$ Decentralized Terminals . . . . . . . . . . . . . . . . . . . . . 91

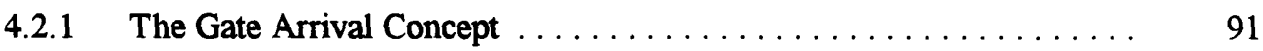

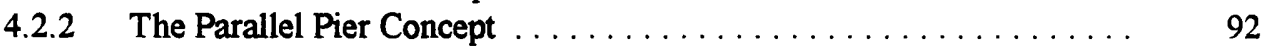

4.2.3 The Satellite Concept $\ldots \ldots \ldots \ldots \ldots \ldots \ldots \ldots \ldots \ldots \ldots \ldots .92$

4.2.3.1 Circular Satellites ...................... 93

4.2.3.2 Rectangular and "T"-Shaped Satellites ............ 94

4.2.4 The Midfield Concept . . . . . . . . . . . . . . . . . . . . 96

5 Estimating Potential Airport Performance

$\begin{array}{ll}\text { Part I : Passenger Convenience Measures } & 100\end{array}$

$5.1 \quad$ Representing the Airport Environment $\ldots \ldots \ldots \ldots \ldots \ldots \ldots \ldots \ldots \ldots$

$5.2 \quad$ Estimating Passenger Walking Distances $\ldots \ldots \ldots \ldots \ldots \ldots \ldots \ldots \ldots \ldots$

$5.2 .1 \quad$ Basic Model . . . . . . . . . . . . . . . . . . . . 106

5.2.2 Estimating Transition Probabilities $\ldots \ldots \ldots \ldots \ldots \ldots \ldots \ldots \ldots, 112$

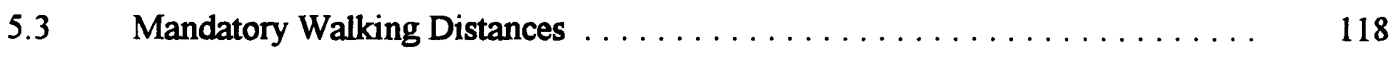

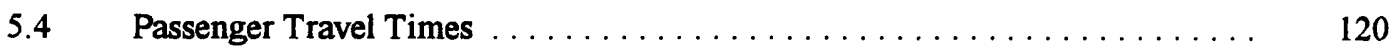

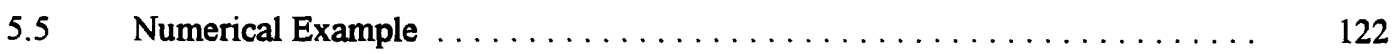

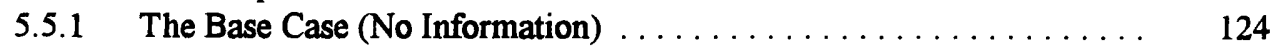

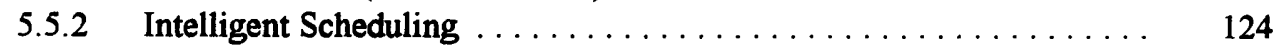

$5.5 .3 \quad$ Aircraft Effects . . . . . . . . . . . . . . . . . . . . 126

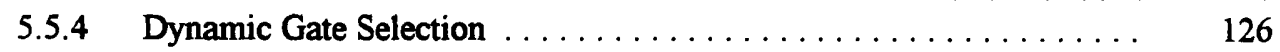

5.5.5 Summary (The Combined Case) $\ldots \ldots \ldots \ldots \ldots \ldots \ldots \ldots \ldots \ldots \ldots$

$6 \quad$ Some Numerical Results 130

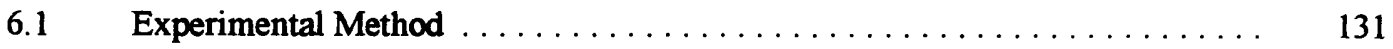

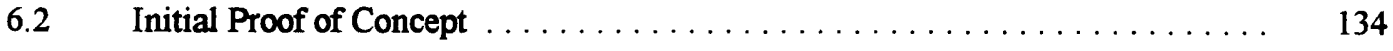

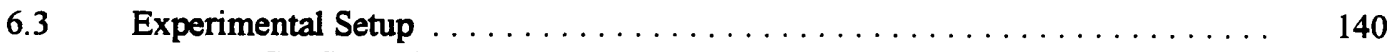

6.3.1 Configurations and Geometries Considered .............. 140

6.3.2 Centralized Concepts .......................... 141

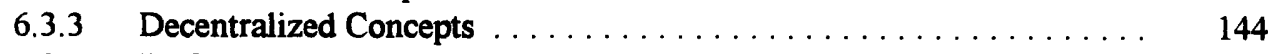

6.3.4 Performance Robustness $\ldots \ldots \ldots \ldots \ldots \ldots \ldots \ldots \ldots \ldots \ldots .144$

$6.4 \quad$ Robustness to Transfer Traffic . . . . . . . . . . . . . . . . . . . . 149

6.4.1 Centralized Configurations . . . . . . . . . . . . . . . . . 149 
6.4.2 Decentralized Configurations ...................... 151

6.4.3 Selecting an Initial Configuration $\ldots \ldots \ldots \ldots \ldots \ldots \ldots \ldots \ldots$

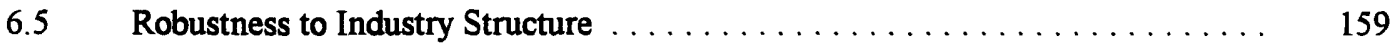

$6.6 \quad$ Robustness to Size (Design for Expansion) $\ldots \ldots \ldots \ldots \ldots \ldots \ldots \ldots \ldots \ldots$

$7 \quad$ Estimating Potential Airport Performance

Part II : Baggage and Aircraft Measures 172

$7.1 \quad$ Baggage Transfer Times . . . . . . . . . . . . . . . . . . . . . . 173

7.2 Estimating Expected Transfer Times for Baggage . . . . . . . . . . . . . . 177

7.2.1 Loading and Unloading Times . . . . . . . . . . . . . . . . 177

7.2 .2 Sortation Times . . . . . . . . . . . . . . . . . . . . . 179

7.2.3 Conveyance and Transport Times $\ldots \ldots \ldots \ldots \ldots \ldots \ldots \ldots \ldots . \ldots \ldots$

7.2.4 General Model for Expected Transfer Time ................. 181

7.2.5 Estimating Baggage Transition Probabilities ............. 182

7.3 Aircraft Taxi Times $\ldots \ldots \ldots \ldots \ldots \ldots \ldots \ldots \ldots \ldots \ldots \ldots \ldots \ldots \ldots$

7.3.1 Factors Influencing Aircraft Taxi Times . . . . . . . . . . . . . . 184

7.3.2 Absolute Distance of Path ... . . . . . . . . . . . . . . . . . . . . 185

7.3.3 Number of Aircraft Turns . . . . . . . . . . . . . . . . . . . . . . . 187

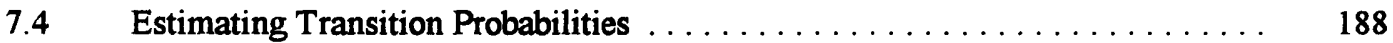

7.4.1 Unconditional Exit Taxiway Probabilities ............... 189

7.4.2 Arrival Gate Probabilities . . . . . . . . . . . . . . . . 193

$7.5 \quad$ Numerical Example $\ldots \ldots \ldots \ldots \ldots \ldots \ldots \ldots \ldots \ldots \ldots \ldots \ldots \ldots \ldots \ldots$

$8 \quad$ Potential Congestion $\quad 200$

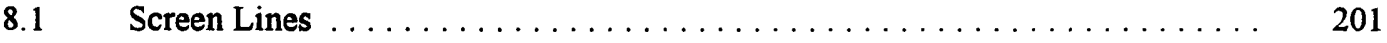

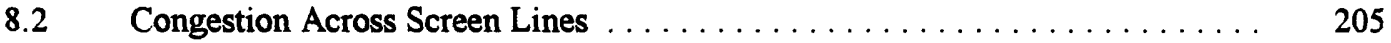

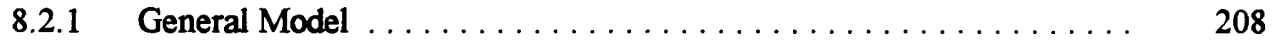

8.2.2 Numerical Example of Pedestrian Flow Analyses . . . . . . . . . . . 209

$8.3 \quad$ Congestion in an Area $\ldots \ldots \ldots \ldots \ldots \ldots \ldots \ldots \ldots \ldots \ldots . \ldots \ldots \ldots$

8.3.1 IATA Level of Service Parameters . . . . . . . . . . . . . . . 210

8.3.2 General Model for Estimating Area Congestion . . . . . . . . . . . . . 214

8.4 Case Study - Boston Logan International (Terminal E) . . . . . . . . . . . . . 217

8.4.1 General Description of Terminal E . . . . . . . . . . . . . . . . . 217

8.4.2 Congestion Across a Line: Required Stairway Widths . . . . . . . 221

8.4.2.1 Other Airline Operations $\ldots \ldots \ldots \ldots \ldots \ldots \ldots \ldots .221$

8.4.2.2 Northwest Operations . . . . . . . . . . . . . . . . . . 224

8.4.3 Congestion in an Area ....................... 226

8.4.4.1 Other Airline Operations $\ldots \ldots \ldots \ldots \ldots \ldots \ldots \ldots, 227$

8.4.4.2 Northwest Operations .................. 230

$9 \quad$ Conclusions and Suggestions for Further Research 232

$9.1 \quad$ Current Decision Support is Inadequate $\ldots \ldots \ldots \ldots \ldots \ldots \ldots \ldots \ldots \ldots$

$9.2 \quad$ Characterizing Different Airport Configurations $\ldots \ldots \ldots \ldots \ldots \ldots \ldots . \ldots . \ldots . \ldots 235$ 


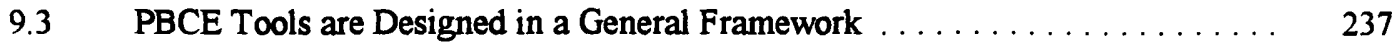

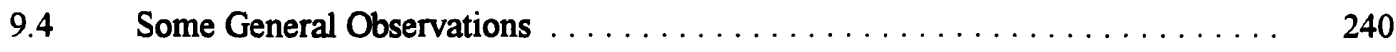

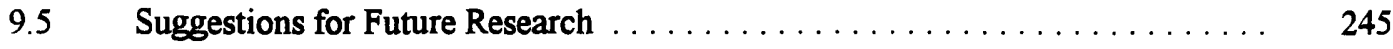

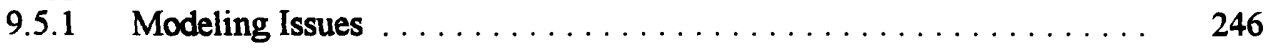

9.5.2 Implementation Issues: A Graphical Interface $\ldots \ldots \ldots \ldots \ldots \ldots 248$

$\begin{array}{ll}\text { Appendix A - Simulation Input Data } & \mathbf{2 5 0}\end{array}$

$\begin{array}{ll}\text { Bibliography } & 252\end{array}$ 


\section{List of Figures}

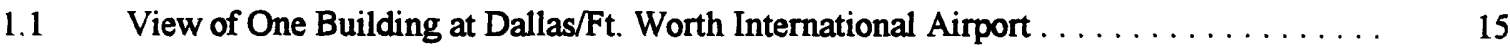

$1.2 \quad$ FAA Flight Service Workload, Actual and Forecast, $1960-2000 \ldots \ldots \ldots \ldots \ldots \ldots .25$

1.3 Comparison in Terms of Broad Range Performance .................... 27

$1.4 \quad$ Position Matrix . . . . . . . . . . . . . . . . . . . . . . . . . . . . 29

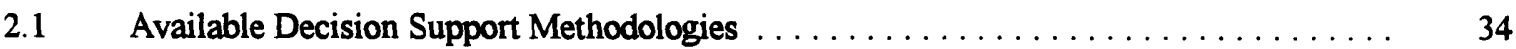

$2.2 \quad$ Sensitivity Analyses through Analytic Techniques . . . . . . . . . . . . . . . . . . . 37

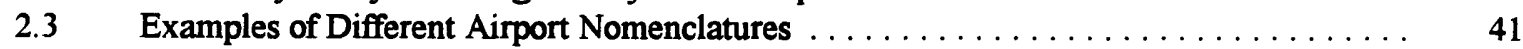

$2.4 \quad$ Decision Support for Initial Configuration Selection $\ldots \ldots \ldots \ldots \ldots \ldots \ldots \ldots \ldots \ldots$

2.5 Decision Support for Detailed Floor Plan Layout $\ldots \ldots \ldots \ldots \ldots \ldots \ldots \ldots \ldots \ldots \ldots$

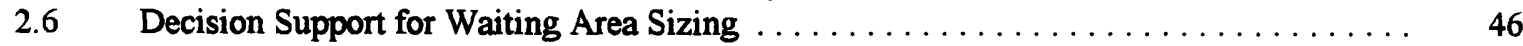

2.7 Centralized and Decentralized Satellite Characterizations $\ldots \ldots \ldots \ldots \ldots \ldots \ldots \ldots$

$2.8 \quad$ Closed-Loop Centralized Hub Terminal . . . . . . . . . . . . . . . . . . . . . . . . . 52

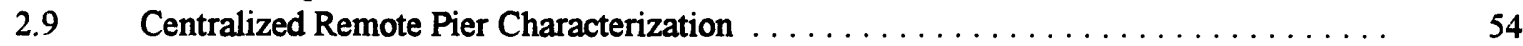

$2.10 \quad$ Graphic Representation of Decision Support $\ldots \ldots \ldots \ldots \ldots \ldots \ldots \ldots \ldots \ldots \ldots \ldots$

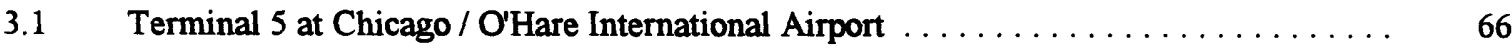

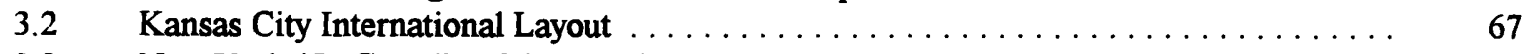

$3.3 \quad$ New York / LaGuardia Main Terminal Layout $\ldots \ldots \ldots \ldots \ldots \ldots \ldots \ldots \ldots \ldots$

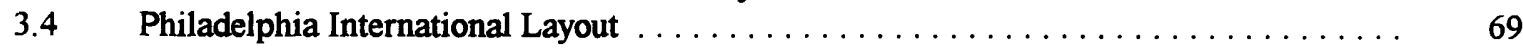

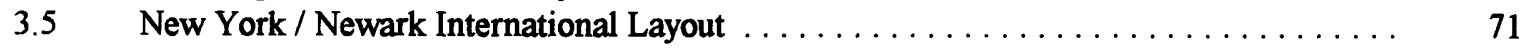

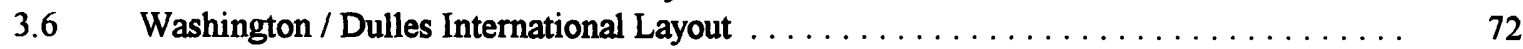

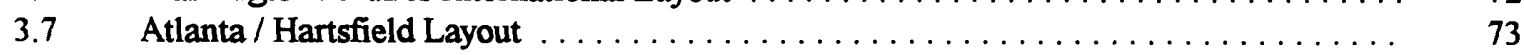

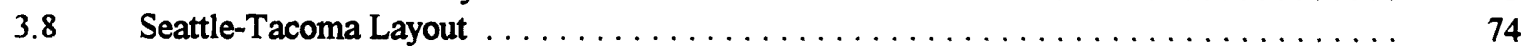

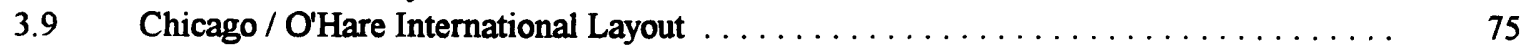

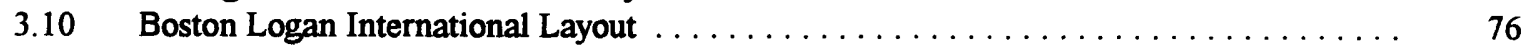

3.11 Proposed Hong Kong / Chek Lap Kok Layout $\ldots \ldots \ldots \ldots \ldots \ldots \ldots \ldots \ldots \ldots$

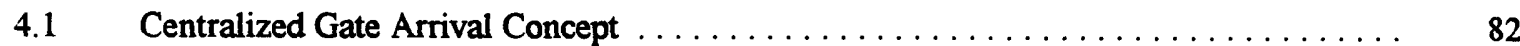

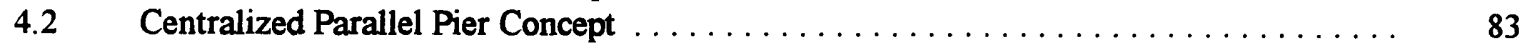

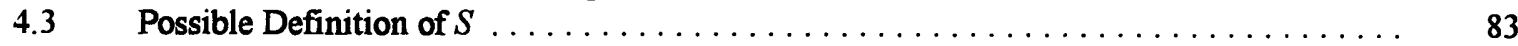

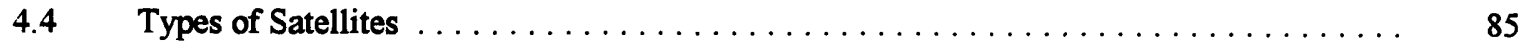

$4.5 \quad$ Centralized Satellite Configuration with Three Circular Concourses . . . . . . . . . 85

4.6 Centralized Satellite Configuration with Two Rectangular Concourses . . . . . . . . . 87

$4.7 \quad$ Parallel Linear Midfield Concept . . . . . . . . . . . . . . . . . . . . . . . . 89

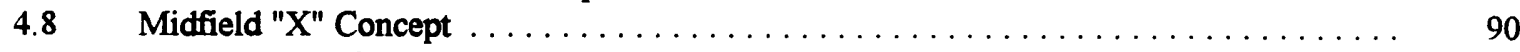

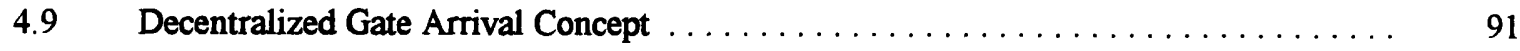

4.10 Decentralized Parallel Pier Concept . . . . . . . . . . . . . . . . . . . . 92

4.11 Decentralized Satellite Construction with Three Circular Concourses . . . . . . . . . . . 93

4.12 Decentralized Satellite Construction with Two "T"-Shaped Concourses . . . . . . . . . . . 95

4.13 Decentralized Midfield Configuration with Two "X" Concourses . . . . . . . . . . . . 96

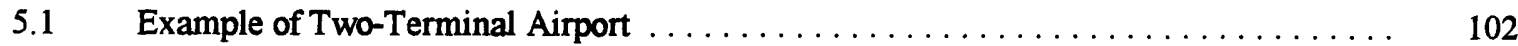

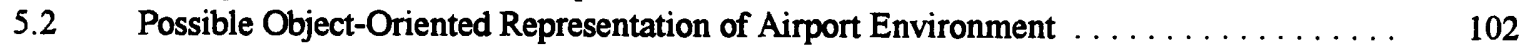

$5.3 \quad$ Possible Daily Demand Profiles . . . . . . . . . . . . . . . . . . . . . . . . 117

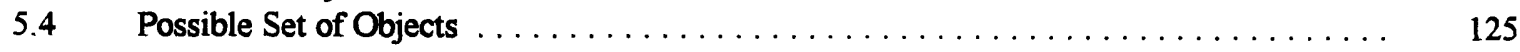

6.1 Performance Profile for Centralized, Circular Satellite Configuration . . . . . . . . . 133

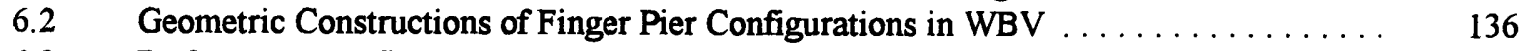

6.3 Performance Profile for Type 3 Configuration $(G=72) \ldots \ldots \ldots \ldots \ldots \ldots \ldots \ldots$ 


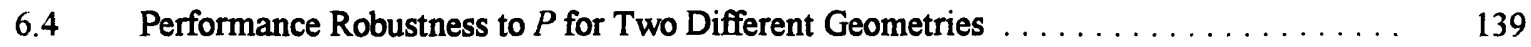

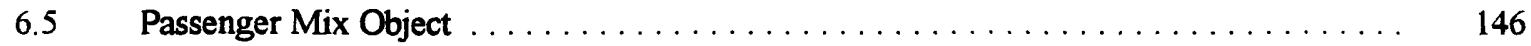

6.6 Profile for Centralized Rectangular Satellite Configuration $\ldots \ldots \ldots \ldots \ldots \ldots \ldots \ldots . \quad 149$

6.7 Profile Lines for Individual Passenger Categories $(P=.5) \ldots \ldots \ldots \ldots \ldots \ldots \ldots$

6.8 Profile for Decentralized Rectangular Satellite Configuration $\ldots \ldots \ldots \ldots \ldots \ldots \ldots \ldots 151$

6.9 Profile Lines for Individual Passenger Categories $(P=.5) \ldots \ldots \ldots \ldots \ldots \ldots \ldots \ldots \ldots$

6.10 Decision Tree for Selecting Most Robust Geometry $\ldots \ldots \ldots \ldots \ldots \ldots \ldots \ldots \ldots \ldots$

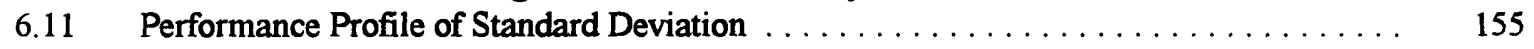

6.12 Performance Profile of Probability of Excess Walking $\ldots \ldots \ldots \ldots \ldots \ldots \ldots \ldots \ldots$

$6.13 P$ vs. $Q$ "Best of the Best" Tableau for Walking Distances Only $(G=24) \ldots \ldots \ldots \ldots$

6.14 Performance Profile for Centralized Parallel Pier Configuration $\ldots \ldots \ldots \ldots \ldots \ldots \ldots 160$

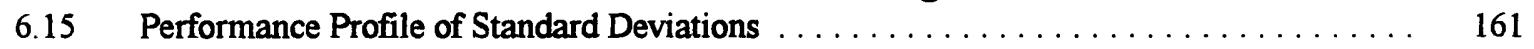

6.16 Performance Profile of Probability of Excess Walking $\ldots \ldots \ldots \ldots \ldots \ldots \ldots \ldots \ldots$

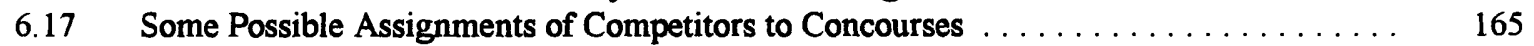

$6.18 P$ vs. $C$ "Best of Best" Tableau for Walking Distances Only $(G=24) \ldots \ldots \ldots \ldots \ldots \ldots$

6.19 Graphical Representation of $(P$ vs. $C)$ Tableau Results $\ldots \ldots \ldots \ldots \ldots \ldots \ldots \ldots \ldots$

$6.20 \quad$ Performance Profile for Centralized Circular Satellite $(G=24) \ldots \ldots \ldots \ldots \ldots \ldots$

6.21 Performance Profile for Centralized Circular Satellite $(G=48) \ldots \ldots \ldots \ldots \ldots \ldots$

$6.22 \quad$ Simplified Decision Matrix for Expansion Decision . . . . . . . . . . . . . . . . . 171

6.23 Decision Tree for Selecting Most Robust Geometry (for Expansion) $\ldots \ldots \ldots \ldots \ldots \ldots 171$

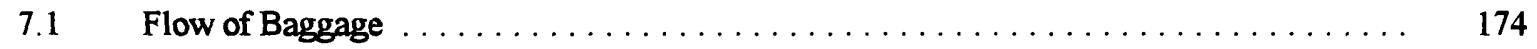

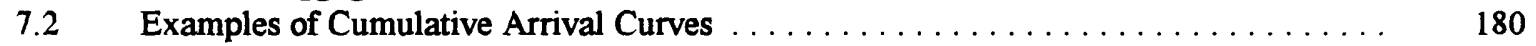

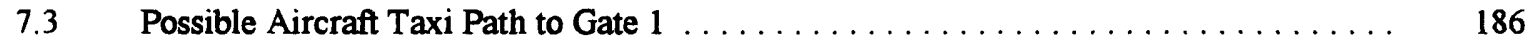

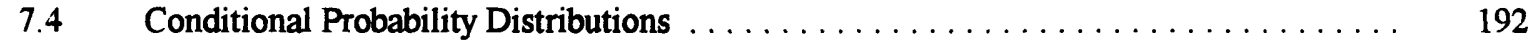

7.5 "X"-Shaped Concourse for Proposed Kuala Lumpur International Airport . . . . . . . . 195

$7.6 \quad$ "Equivalent" Parallel Linear Concourse . . . . . . . . . . . . . . . . . . . . . 197

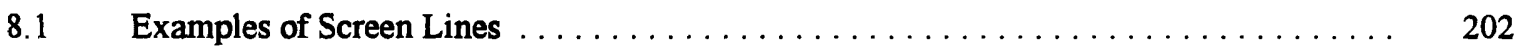

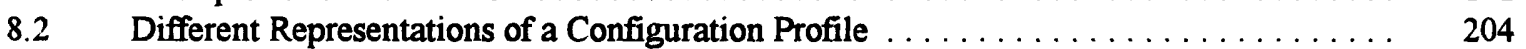

8.3 Flow-Volume Relationships for Pedways . . . . . . . . . . . . . . . . . . . . . . . . . . . 207

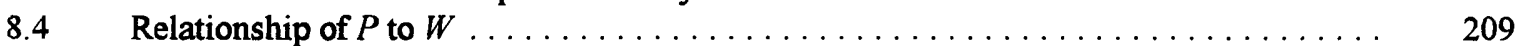

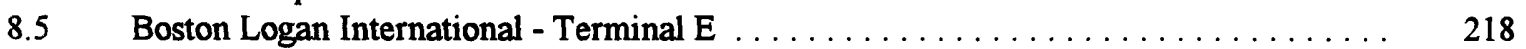

8.6 Northwest Portion of Terminal E . . . . . . . . . . . . . . . . . . . . . . . . . . . . . 219

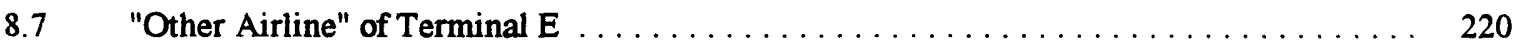

8.8 Simulated Cumulative Arrival Curve for LH $423 \ldots \ldots \ldots \ldots \ldots \ldots \ldots \ldots \ldots \ldots . \ldots \ldots$

8.9 Simulated Cumulative Arrival Curve for All "Other Airline" Flights . . . . . . . . . . 223

8.10 Arrival Rate Curve for Individuals on all "Other Airline" Flights . . . . . . . . . . . 223

8.11 Simulated Cumulative Arrival Curve for All Northwest Flights . . . . . . . . . . . 225

8.12 Arrival Rate Curve for All Northwest Flights .................... 226

8.13 Arrival and Departure Rate Curves for "Other Airline" Individuals . . . . . . . . . 228

8.14 Cumulative Arrival and Departure Curves for "Other Airline" Individuals . . . . . . . . 228

8.15 Total Individual Accumulation for "Other Airline" Flights . . . . . . . . . . . . . . 229

8.16 Total Individual Accumulation for Northwest Flights $\ldots \ldots \ldots \ldots \ldots \ldots \ldots \ldots \ldots$

9.1 Characteristic Performance Profile for Centralized Configurations . . . . . . . . . . 241

9.2 Characteristic Performance Profile for Decentralized Configurations . . . . . . . . . 242 


\section{List of Tables}

$1.1 \quad$ Elapsed Time Between Planning and Completion of Airports . . . . . . . . . . . 14

1.2 The Architect's View of Airport "Terminal" Design . . . . . . . . . . . . . . . . 20

1.3 Average Errors in the Forecasts of Air Traffic of the

2.1 FAA Recommended Relationships for TPHP Computations from Annual Figures . . . . 44

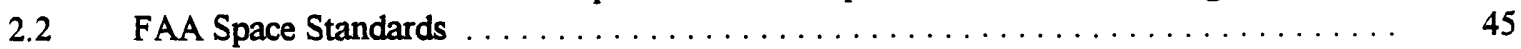

2.3 Analytic Studies have Considered Only a Limited Range of Elements . . . . . . . . 55

4.1 Parameters Used in Representing Centralized Configurations . . . . . . . . . . . . . 98

4.2 Additional Parameters Used in Representing Decentralized Configurations . . . . . . . . 99

5.1 List of Objects and Attributes Used to Represent the Airport Environment . . . . . . . 103

5.2 Preliminary Data for Sample Airport Given "Base Case" (No Information) . . . . . . . 123

5.3 Transition Probability Matrices Given Additional Information ............... 128

5.4 Summary of Estimates for Expected Walking Distances . . . . . . . . . . . . . . . . . 129

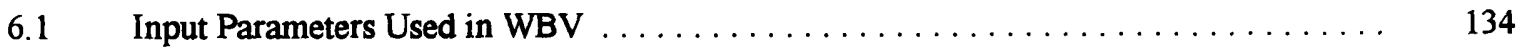

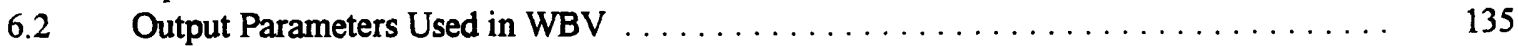

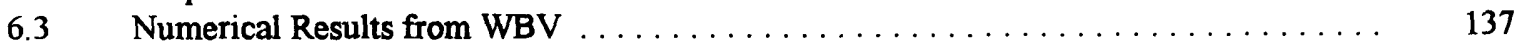

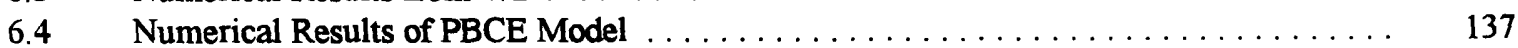

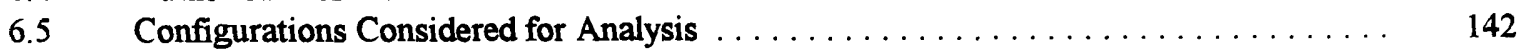

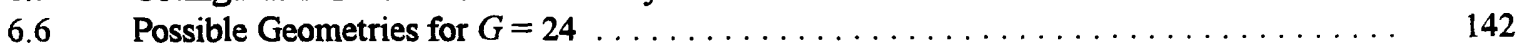

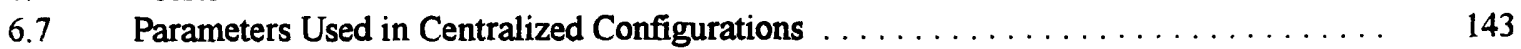

$6.8 \quad$ Parameters Used in Decentralized Terminal Configurations . . . . . . . . . . . . . 145

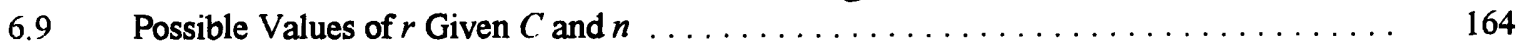

7.1 Distance and Required Turn Data for "X"-Shaped Concourse . . . . . . . . . . . 196

7.2 Distance and Required Turn Data for "Equivalent" Parallel Linear Concourse . . . . . . . 198

8.1 Level of Service Design Standards for Walkways and Stairways . . . . . . . . . . . 208

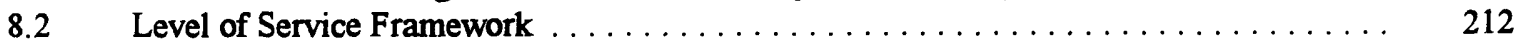

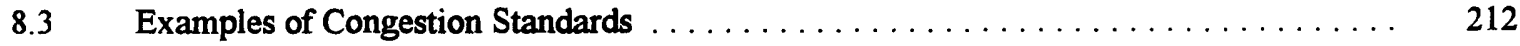

A.1 Input Flight Schedule (Thursday, August 1988) $\ldots \ldots \ldots \ldots \ldots \ldots \ldots \ldots \ldots \ldots \ldots$

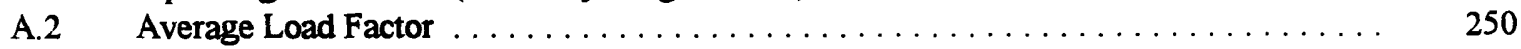

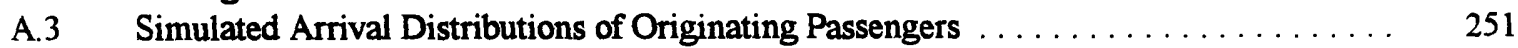

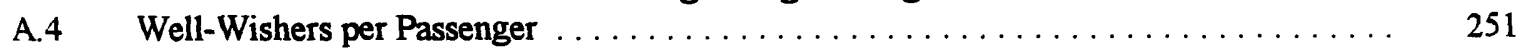

A.5 Originating versus Transferring Passengers $\ldots \ldots \ldots \ldots \ldots \ldots \ldots \ldots \ldots \ldots \ldots \ldots \ldots$ 


\section{Chapter 1}

\section{Introduction}

\section{1 - Motivation}

Errors in the initial stages of design for airport passenger buildings can be very expensive. From a purely economic perspective, the prevention of design errors for a single airport terminal can amount to tens of million dollars in savings. It is believed, for instance, that avoidable design errors at the Air France terminal at Paris/Roissy (Aerogare 2) resulted in an additional $\$ 75$ million in expenses [de Neufville and Grillot 1982]. More recently, the new Terminal 2 at the Frankfurt/Main Airport has had to undergo significant design changes because Lufthansa, the dominant carrier there, decided not to separate its domestic and international operations between two buildings, choosing instead to centralize all its operations in the old Terminal 1 [Birch 1993].

The preceding examples are not isolated incidences. The design of many other airport terminals appeared sound at their inception yet, over time, became inappropriate for future environments. Such failures cannot be attributed solely to oversights on the part of design team members. Instead, it is more likely that the design process itself is to blame, and that errors by team members arise from an inappropriate concept of design needs. 
The planning and design process for airport passenger buildings is difficult due to the dynamic nature of the system. Airport master plans are typically conceived many years before actual construction is completed, and during that time major changes in the air transport system can occur which dramatically change the composition of passenger traffic. Table 1.1 illustrates some examples of the length of time that elapses between the initial planning and the completion of airport construction.

Table 1.1: Elapsed Time Between Planning and Completion of Airports

\begin{tabular}{|c|c|c|c|}
\hline Airport & Planning Start & Date Opened & Years \\
\hline Cologne/Bonn & 1962 & 1970 & 8 \\
\hline Dallas/Ft. Worth & 1965 & 1973 & 8 \\
\hline Paris Roissy-en-France & 1964 & 1974 & 10 \\
\hline Seattle-Tacoma & 1967 & 1980 & 13 \\
\hline Frankfurt/Main & 1950 & 1972 & 22 \\
\hline Munich 2 & 1954 & 1992 & 38 \\
\hline
\end{tabular}

Source : [Blankenship 1974]

The average lead time for the above examples is over 16 years, with the longest (Munich 2) spanning 38 years. Much can happen over that time to change the fundamental character of aviation demand. Therefore, it is critical to consider in the overall design philosophy how a particular configuration will perform under conditions of change. The Dallas/Fort Worth experience illustrates the problems that can occur when change is not considered in the selection of the initial passenger building configuration.

\section{The Dallas/Fort Worth Experience}

When the Dallas/Forth Worth Airport was being designed, the U.S. interstate airline industry was a completely regulated environment (under the jurisdiction of the Civil Aeronautics Board), and the use of passenger security checkpoints (with X-ray machines and metal detectors) was not as widespread as it is today. Most airlines flew point-to-point route networks, and thus the bulk of airport traffic consisted 
of originating and terminating passengers. For such passengers, minimizing the distance from curbside to their departure gate is an important design criterion. One configuration which meets this criterion is the gate arrival concept (Figure 1.1), which can house many entrances/exits along the curbside frontage. Originating passengers are assumed to enter the terminal building at an entrance very near their departure gate. Thus walking is reduced to the width of the building itself, provided they do not have to deviate from their path to pass through security.

Economic deregulation in the United States in 1978 changed the U.S. airline industry significantly. Most notably, airlines were given the freedom to serve any city-pair market in the domestic system without having to file for route authority with the C.A.B. Many carriers took advantage of this freedom and established hub-and-spoke route networks. By utilizing a hub-and-spoke system, airlines could provide a better level of service in terms of schedule frequency to each city-pair market (with the same number of aircraft) than they could flying a predominantly point-to-point network.

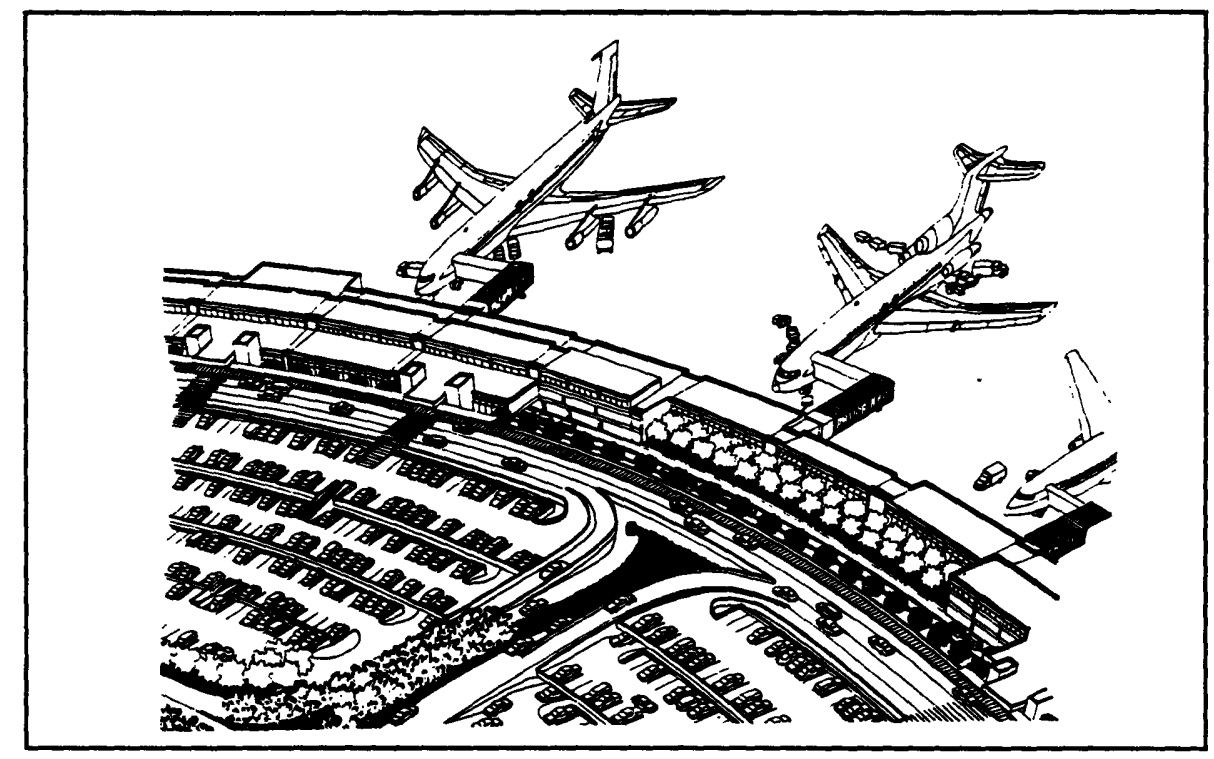

Figure 1.1 : View of One Building at Dallas/Ft. Worth International Airport ${ }^{1}$

After deregulation, American Airlines established their largest hub in Dallas/Ft. Worth. This move significantly changed the nature of passenger traffic there, from primarily originating and

\footnotetext{
${ }^{1}$ Source: [Blankenship 1974], p. 119.
} 
terminating passengers to a high proportion of transfer traffic $(65 \text { percent in 1992) })^{2}$. For transfer passengers, the linear design of the gate arrival concept poses problems when trying to make connections from one end of the airport to the other. In short, the airport (designed to minimize walking distances) is now notorious for its excessive connection distances, for some close to one half of a mile.

Further difficulties were encountered at Dallas/Ft. Worth with the introduction of security checkpoints. In theory, the gate arrival concept provides short walking distances for originating passengers; however, those passengers now had to pass through security stations before reaching the departure area. To keep walking distances to a minimum, security stations would be needed at every entrance in order to prevent passengers from having to divert significantly from their direct path. Such duplication of security stations is expensive, and as a result most of the security checkpoints were closed in order to centralize security operations. The result of this action was that many originating passengers no longer had a direct path from their entrance to their departure gate, defeating the purpose of the gate arrival design for the passengers it was originally intended to serve.

Perhaps the most important lesson to be learned from this experience is that designing an airport passenger building configuration based on a singular notion for the future (e.g. predominantly originating and terminating traffic) can result in major operational problems in times of change. What is needed is a re-examination of the design process in order to improve the quality of decisions being made. The following section investigates in greater detail the shortcomings inherent in the process of airport passenger building design.

\section{2 - Airport Passenger Building Design}

The planning and design of an airport is a complex process, involving the synthesis of many dynamic and interdependent systems. It is, in fact, just one component of the larger process of planning an entire airport system, which Horonjeff and McKelvey [1983] have defined as :

\footnotetext{
${ }^{2}$ Source : U.S. Department of Transportation Origin and Destination Survey, 1992
} 
a representation of the aviation facilities required to meet the intermediate and future needs of a metropolitan area, region, state, or country. It presents the recommendations for the general location and characteristics of new airports and the nature of expansion for existing ones. It includes the timing and estimated costs of development and relates airport system planning to the policy and objectives of the relevant jurisdiction. Its overall purpose is to provide the basis for definitive and detailed airport planning, such as the airport master plan. [p.146]

The airport system plan serves as an overall framework under which specific airport programs are developed to serve the short- and long-term requirements of the surrounding aviation community. Individual airport development is accomplished through the development of airport master plans.

Airport Master Plans provide specific guidelines for present and future construction so as to meet the specific needs of the air transport system, while remaining compatible with the environment, the community, and the transportation infrastructure already in place. As defined by the United States Federal Aviation Administration, the selection of the initial configuration of the airport passenger buildings is generally accomplished during the development of the Terminal Area Plan, after the demand/capacity analyses and the facility requirement studies are completed [FAA 1971].

Prevalent in the current practice of selecting a configuration of passenger buildings is the notion that performance can be adequately addressed by considering only a single forecast for the future, and that performance can be described using only a limited number of criteria. Such a philosophy neglects the possibility that the airport may witness major changes in traffic patterns over the course of its useful life. As we have seen in the Dallas/Ft. Worth case, this philosophy can lead to significant operational difficulties if the potential for change is ignored.

\subsection{1 - Single Forecast for the Future : The Airport Building as a "Terminal"}

Much of the problem in the current design philosophy stems from the traditional view of airport buildings as "terminals". As such, an airport building is seen as the end (or beginning) of a passenger's journey and acts as a gateway to the surrounding city or community. According to the FAA Advisory

Circular on Airport Master Plans [1971], 
the objective of the Terminal Area Plan should be to achieve an acceptable balance between passenger convenience, operating efficiency, facility investment, and aesthetics ... the terminal area should afford the passenger orderly and convenient progress from his (or her) automobile or public transportation through the terminal to the aircraft. [p. 51]

Thus, the principal objective of an airport building (according to the Advisory Circular) is to provide a high level of convenience only for originating (and similarly, terminating) passengers. More importantly, the simple forecast of type implied by this passage is that the dominant type of traffic at the airport is, and always will be, originating or terminating.

The explicit passenger forecast specified in the Advisory Circular relates to the volume of traffic predicted, rather than the mix. Specifically, the FAA requires a single forecast, passengers enplaned annually (for short, intermediate, and long range time frames), when producing a master plan. According to the Advisory Circular [1985],

The idea is to forecast the different elements of aviation demand, compare that demand over time with the capacity of airports' various facilities, and to identify the time when new or expanded airport facilities may be necessary. [p. 22]

Consequently, errors in the forecast are assumed to relate only to time, i.e. over- or underestimating when the specified volume of originating and terminating traffic will materialize [Maldonado 1990].

In the case of airport master planning forecasts, the costs of errors in the forecasts are related to the timing for investments to be made to meet new demands at the airport. [FAA 1985, p. 21]

Thus, the above passages suggest that the forecast for passenger traffic as specified by the Advisory Circular is one in which the type does not change (i.e. primarily originating and terminating) and the volume is expressed only in terms of a single statistic (annual passengers enplaned). This notion reinforces the idea of the airport as a "terminal", where forecast errors pose problems only for the staging of expansion, not for the selection of the overall concept.

The actual design of the airport buildings is often the responsibility of one or a team of architects, who use a different set of criteria to describe the objectives of the terminal area -- they tend to stress 
artistic and aesthetic merits rather than operational features when describing the "performance" of buildings. Yet, as Table 1.2 illustrates in passages taken from architects (when describing various airport projects), the pervading theme of the airport as a "terminal" also exists in the architectural community. Indeed, the issue of a passenger's "experience" is an important one, as it is often the first or only impression he or she gets of the surrounding community. Again, however, the passages suggest that even architects recognize the role of the airport as a "gateway to the city" or in terms of a passenger's "entry" or "arrival". This view tacitly assumes the same "static" forecast of the type of passenger traffic that the Advisory Circular suggested previously.

The result of this perspective is that many smaller design firms often rely on fairly conventional, formulaic designs of a few passenger building concepts, as prescribed in various reference manuals and handbooks (e.g. The LATA Airport Terminals Reference Manual and The Apron and Terminal Building Planning Manual, by the Ralph M. Parsons Company). This "cookbook"-type approach largely neglects the possibility of changes in the functional requirements of buildings (both short- and long-term), and as a result inflexibility can become embedded into the design. The following passages (about the new Stansted Airport in England) illustrate the discord between form and function that can result when the operational features of a building are not explicitly incorporated into the initial design process :

But, of course, every airport has to house a mass of low level accommodation, ranging from check-in desks to customs and passport control booths, with shops, restaurants, bars and all manner of small service spaces between. From the first, these were bound to conflict with and clutter the simplicity of the main idea.

The architects' response has been to impose a very strong order on the lower structures which is completely independent of the larger order of the building itself.

[Davey, p. 44]

So it is refreshing to find that at Stansted the retailing is a concession in the exact sense of the term. It is tolerated. It is allowed its place but has been subjected to iron architectural control. Stansted is a triumphant affirmation of the principle that air travel is about higher things. [Best, p. 59]

Source: The Architectural Review, May 1991 
Table 1.2: The Architect's View of Airport "Terminal" Design

\begin{tabular}{lll}
\hline Source & Airport & Description \\
\hline
\end{tabular}

Valerie E.

Koukoutsi,

Arrival in Arabia, 1989.

Helmut Jahn, Airports, 1991.

The Economist, March 23, 1991.

Peter Davey, The Architectural Review, May 1991.
King Abdulaziz International

Airport,

Saudi Arabia

United Airlines

Terminal 1, O'Hare International Airport, Chicago
Kansai Airport, Japan

Stansted Airport, England
The prevailing concept is integration of the South Terminal with the airport and the city. The Terminal is a part of the city. Our approach provides the traveler with an environment which can be considered his/her first or last stop within the country. It is the host place of the caravans. [p. 12]

The subtle transparency of the light, with its reflections, the colors and the materials influence the passenger areas such that they radiate peace and quiet and provide a welcome contrast to the hectic of traveling. These contrasts are multilayered. Calm alternates with activity and excitement. The building is functional and rational as well as imaginative. Technology confronts the lyrical and romantic. The United Airlines Terminal is as much a gateway to the world as it is to the city of Chicago. [p. 32]

The traveler will land and leave enchanted by the peculiar magic of all islands. The idea is to create an image of virgin coastline of an island forest through which one is drawn towards the terminal. Mr. [Renzo] Piano believes that the architecture of the airport is about the "movement patterns" of people, and "the atmospheric elements of the space -- light, sound, air movement" can be deployed to help passengers find their way about. At Kansai, he has added a poetic dimension by introducing into the building two longitudinal spines of landscaped gardens: what he calls "valleys full of daylight, penetrated by nature." [p. 98]

The experience at Stansted can be analysed in archetypal terms. Such a description might run something like this: the entry is through a grove (or it will be when the trees on the sides of the railway cutting have grown up). The arrival is in a cave from which the passenger gradually ascends to a huge luminous tent. After passing through clearly organised stages of preparation, and arriving at a point where the initiate can contemplate the heavens, he is plunged underground again to arrive at a jetty whence he boards his aerial vessel. [p. 45] 
This lack of consideration for the operational needs of an airport can largely be attributed to inexperience, as the number of architectural and engineering firms having the opportunity to design more than one or two airports is small. Two complementary factors contribute to this: first, the number of new airport terminals being built today is relatively small and second, design contracts are often steered toward local firms. This industry-wide lack of experience for all but a few very large design teams offers little chance for improvement (through practice alone) in the way designers view airport buildings; namely, as "terminals".

Increasingly, however, airport buildings are being used as more than simply beginning or ending points. According to de Neufville [1994],

they are becoming transfer points, airports at which a very large percent of the passengers use the airport to change from one aircraft to another. The fraction of airport passengers transiting through the transfer airports is frequently more than half. At some airports as many as 3 out of every 4 passengers land merely to continue their trip on another flight within one or two hours.

Thus, the view of the airport building as merely a terminal, subject to a single or static forecast for the future, is becoming inadequate. Instead, it should be viewed as a "passenger building", where the volume and type of traffic (originating/terminating vs. transfers) may change significantly over time.

\subsection{2 - Single (or Few) Performance Criteria}

In addition to viewing the future in terms of a single, static forecast as to the volume and type of traffic, current practice also tends to use only a limited number of operational characteristics (often just one) by which to assess the potential performance of different passenger building configurations. For example, the following passage [Wilbur Smith Associates 1988] discusses passenger walking distances as one of only three measures for the level of service : 


\subsubsection{Walking Distances}

The goal for maximum passenger walking distance for the New Denver International Airport is 1,000 feet, gate to gate or from curb to gate. [p. I-4].

The other two measures, minimum connection times and design capacity, were also described in terms of single statistics. The master plan for the Kuala Lumpur International Airport includes the following justification for the selection of four "X"-shaped concourses:

\section{Walking Distance}

One of the reasons for choosing the large, centralised terminal concept for $\mathrm{KL}$ International Airport was to provide a better service for transfer passengers. Table 4.9 compares the maximum walking distance possible for a passenger transferring from an international to international flight.

Table 4.9 : Maximum Waking Distance for International Transfer Passengers

\begin{tabular}{llll}
\hline & $\begin{array}{l}\text { Don Muang } \\
\text { [Bangkok] }\end{array}$ & $\begin{array}{l}\text { Changi } \\
\text { [Singapore] }\end{array}$ & $\begin{array}{l}\text { KLIA } \\
\text { [Kuala Lumpur] }\end{array}$ \\
$\begin{array}{l}\text { Maximum } \\
\text { Walking Distance }\end{array}$ & $1250 \mathrm{~m}$ & $1500 \mathrm{~m}$ & $600 \mathrm{~m}$ \\
\hline
\end{tabular}

Source : [Anglo Japanese Airport Consortium 1992], p. 76.

The above passages highlight the shortcomings of the current state of decision support for assessing the potential performance of different passenger building configurations. First, when operational comparisons are made, they are frequently done using a single dimension of performance (such as passenger walking distances), and a single statistic to describe that dimension (i.e. maximum or mean walking distance). Little if any attention is given to the distribution of performance measures, including for example, the variability of walking distances or the number of passengers expected to walk the maximum distance. The second and perhaps more important shortcoming of the current approach is that comparisons made using the single dimension of performance are done for a single (often unspecified) set of conditions, rather than for a number of different scenarios. 


\section{3 - Robustness in the Design of Airport Passenger Buildings}

Good design practice dictates that "product" performance be thought of in terms of robustness, or how something will perform over a range of different conditions, rather than simply the expected one. In a manufacturing context, this practice translates to the effort to reduce product failure in the field due to uncontrollable and often unpredictable environmental disturbances (or variability) [Byrne and Taguchi 1987]. Such disturbances may produce conditions that deviate significantly from the expected case, and thus consistent performance over a broad range of possible circumstances becomes paramount. Using television sets as an example,

Customers would be dismayed if the [television's] picture degraded every time they turned up the volume. They would reject a TV that developed snow and other annoying "noises" when afflicted by nasty operating conditions, which are themselves considered noises. ${ }^{3}$

Robust quality can thus be thought of as the ability of a product to exhibit consistent performance over a variety of possible, often uncertain conditions, rather than good performance in the expected case, and poor performance under all others. Robustness becomes increasingly important when the uncertainty in the future is high.

In the context of airport planning, environmental disturbances are characterized by "massive uncertainty" [de Neufville 1976]. Changes in the air transportation environment can take many forms including changes in the volume and type of passenger traffic, the competitive structure of the industry, and the technological advances made in aircraft and air traffic control. Each of these factors can, in turn, be influenced by larger economic and political issues. Thus, when forecasting future conditions in the air transportation industry, we can be certain only that whatever the forecast is, it will be incorrect.

The inability to predict future conditions accurately makes the selection of a robust configuration critical to the long term success of the airport. Robustness, however, is innate, and cannot be improved by more accurate predictions of the future. According to Taguchi and Clausing,

\footnotetext{
3 Source: [Taguchi and Clausing 1990], p. 69.
} 
The strength of a product's signal, hence its robustness, is primarily the responsibility of designers. Quality is a virtue of design. An inherent lack of robustness in product design is the primary driver of superfluous expenses. ${ }^{4}$

The above passage applies as well to airports as it does to television sets. Robust quality in the context of airport passenger buildings should thus be thought of as a function of good design. The selection of the initial configuration should be a strategic decision, one that considers the ability of the chosen concept to cope with major changes in the aviation environment, rather than relying on the tactical decisions made by operators to adapt an undesirable configuration (often at great expense) each time conditions change. In other words, "the robustness of products is more a function of good design than of off-line control."5

Moreover, performance should be thought of as a function of many variables instead of just one. Rather than trying to achieve an "optimal" configuration based a single forecast (and measured by a single dimension of performance), the design strategy in place should consider performance as a multidimensional construct, encompassing a variety of possible futures in the selection process. The following section describes this idea which we call "broad range, multiple criteria performance".

\section{4 - Broad Range, Multiple Criteria Performance}

As demonstrated in the Dallas/Forth Worth case, a design strategy based on the idea that performance is one-dimensional, adequately addressed by a single forecast value, can lead to poor decisions in times of major change. It is difficult, if not impossible, however, to predict changes such as deregulation and the effects these changes will have on the future of aviation demand.

As mentioned previously, aviation forecasting is at best an inexact science, due to the massive uncertainty associated with the factors typically used to predict future aviation demand [de Neufville 1976]. Examples include various economic indicators, predictions about the future patterns of airline service, and technological advances in transport aircraft and air traffic control. In addition, many

${ }^{4}$ Ibid., p. 65.

5Ibid., p. 65. 
Table 1.3 Average Errors in the Forecasts of Air Traffic of the Federal Aviation Administration, $1958-71^{6}$

\begin{tabular}{|c|c|c|c|c|c|c|c|}
\hline \multirow[t]{2}{*}{$\begin{array}{l}\text { Nature of } \\
\text { Traffic }\end{array}$} & \multirow[t]{2}{*}{ Type of Traffic } & \multicolumn{6}{|c|}{$\begin{array}{l}\text { Percent Error which is Exceeded Half the Time } \\
\text { after Each Year }\end{array}$} \\
\hline & & $\begin{array}{l}\text { One } \\
\text { Year }\end{array}$ & $\begin{array}{l}\text { Two } \\
\text { Years }\end{array}$ & $\begin{array}{l}\text { Three } \\
\text { Years }\end{array}$ & $\begin{array}{l}\text { Four } \\
\text { Years }\end{array}$ & $\begin{array}{l}\text { Five } \\
\text { Years }\end{array}$ & $\begin{array}{c}\text { Six } \\
\text { Years }\end{array}$ \\
\hline \multirow{3}{*}{ International } & Passengers & 8.0 & 10.9 & 14.0 & 14.0 & 15.1 & 15.8 \\
\hline & Pax-Miles & 7.2 & 10.9 & 13.8 & 15.7 & 16.7 & 19.5 \\
\hline & Average & 7.6 & 10.9 & 13.9 & 14.9 & 15.9 & 17.6 \\
\hline \multirow[t]{3}{*}{ US Domestic } & Passengers & 2.9 & 6.2 & 11.2 & 14.1 & 18.3 & 20.4 \\
\hline & Pax-Miles & 2.9 & 6.0 & 10.6 & 14.5 & 18.3 & 21.8 \\
\hline & Average & 2.9 & 6.1 & 10.9 & 14.3 & 18.3 & 21.1 \\
\hline
\end{tabular}

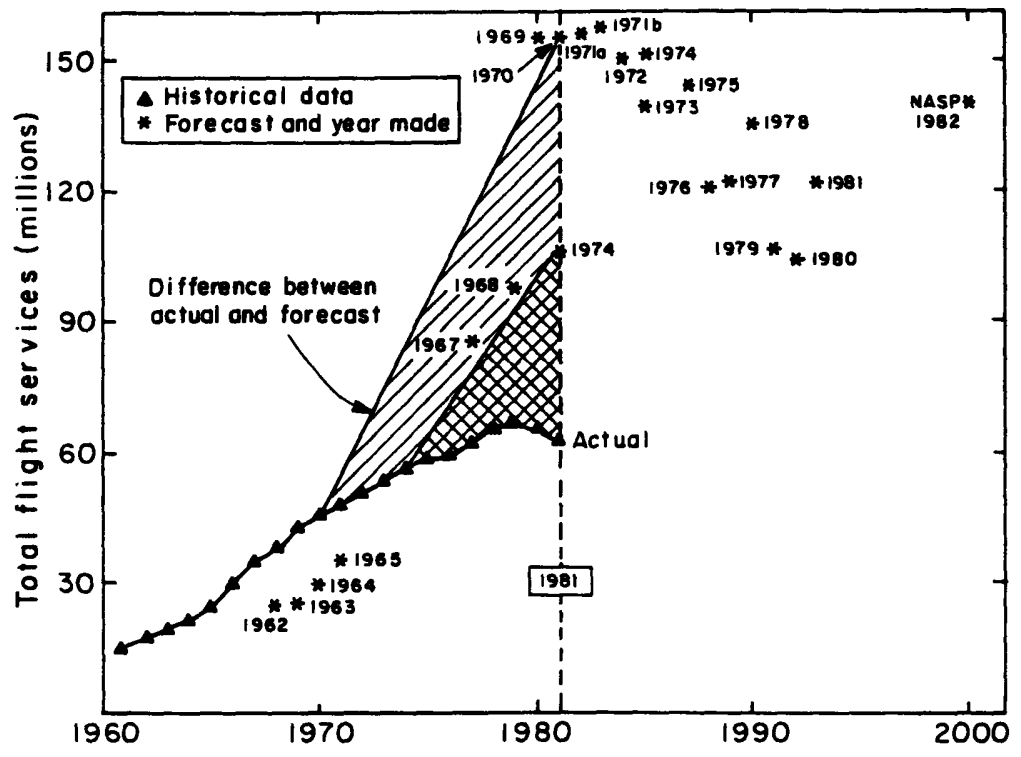

Figure 1.2 : FAA Flight Service Workload, Actual and Forecast, 1960-2000 (Source : U.S. Office of Technology Assessment) ${ }^{7}$

6 Source: [de Neufville 1976], p. 47.

${ }^{7}$ Source: [de Neufville 1990], p. 39. 
unforeseen and "unpredictable" circumstances, such as increased occurrences of terrorism and acts of war, can dramatically influence aviation demand within a short period of time. Table 1.3 and Figure 1.2 are just two examples which illustrate the historical discrepancies that exist between actual and forecasted aviation activity.

\subsection{1 - Broad Range Performance as a Design Objective}

Due to the uncertainty associated with the forecasts for aviation demand, it is unlikely that any one forecast value (for a particular measure) will adequately capture what is likely to happen over the entire life of an airport. Figure 1.3 illustrates the inherent limitations involved in considering only a single forecast value when making comparisons among configuration alternatives. The vertical axis represents performance as measured by some quantifiable criterion, and the horizontal axis represents the range of possible conditions over which performance can be measured. In the context of airport planning, forecast errors can be associated with measures such as the volume of passenger traffic, the mix of traffic (i.e. originating/terminating vs. transfers), as well as the competitive structure of the airline industry (e.g. the number of competitors and their relative market strengths).

If we were certain that the particular forecasted conditions would materialize and never change, the selection of the most appropriate configuration would be simple -- Configuration 1 outperforms Configuration 2 at the specific forecast level. If, however, the forecast conditions do not materialize, or subsequently change over time, the choice of Configuration 1 becomes less desirable -- its performance degrades significantly as we deviate from the forecast value. Configuration 2 , however, is more robust to change, i.e. it performs reasonably well over a wider range of conditions, rather than performing very well over only a limited range. Rather than emphasizing a single statistic to describe performance, such as the mean walking distance or the mean connection time for a single condition (e.g. a given level of transfer traffic), it is important to explore the behavior of that measure over a broad range of possible futures. 


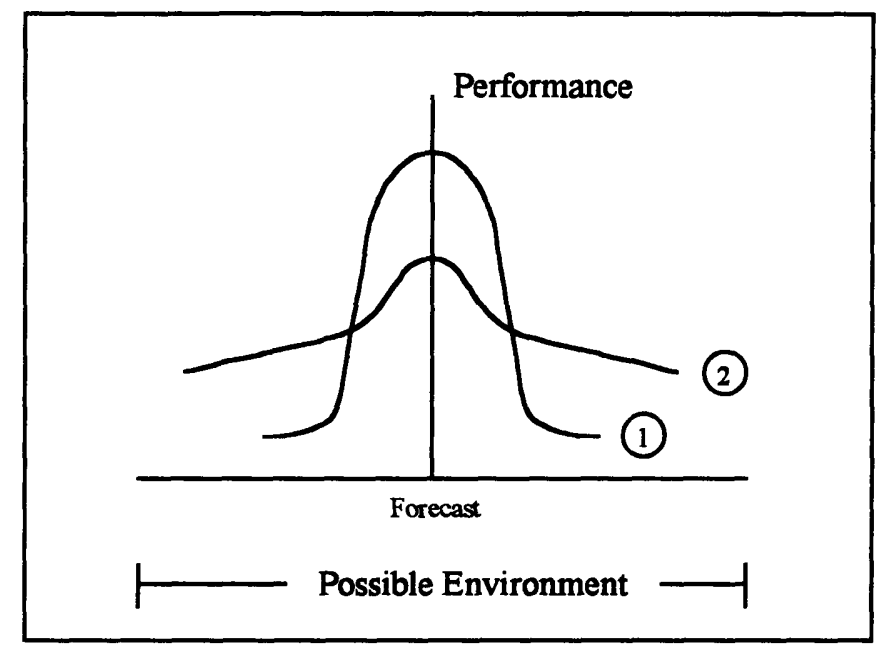

Figure 1.3 : Comparison in Terms of Broad Range Performance

\subsection{2 - Multiple Dimensions of Performance for an Airport Passenger Building}

The topic of airport passenger building performance is one of much study and debate. Lemer [1990] provides a discussion of the characterization and measurement of different metrics of performance for airport passenger buildings. In his report he identifies specific performance measures from the perspectives of the three primary users of airports; namely airport operators, airlines, and passengers.

From the airport owner's viewpoint, performance can be assessed by such measures as operational effectiveness, efficiency, risk, finances and functionality. Quantitatively, operational effectiveness can be measured in terms of passengers and baggage served per unit time, baggage service reliability over time, and flight ground delays. Efficiency can involve such metrics as gate, space, and labor utilization, as well as power and fuel consumption. Risk at an airport can encompass many issues including the effectiveness and accuracy of security checkpoints, the level of crime, and the containment of hazardous materials (in the case of a fire, for example). Functionality, as measured by service reliability and maintainability, may influence lease terms made by airport owners with the airlines, who themselves have standards of performance they must maintain in order to remain competitive. 
Airport performance from the viewpoint of the airlines can be measured by such metrics as operational effectiveness, station costs, and corporate image. Such issues as aircraft turnaround times, flight service times, baggage transfer reliability and passenger service times are all at least to some degree dependent on individual airport characteristics. Thus, the perceived performance of the airline is directly related to the operational performance of the airport itself. Leases between the airlines and airport authorities make corporate image an increasingly important factor in measuring airport performance. Dominance of a particular carrier at an airport terminal creates a strong association between a passenger's perception of the terminal area itself and the host airline. It is thus in the best interests of the airline to keep facilities clean and functioning efficiently.

From a passenger's perspective, the issue of airport performance is a highly subjective one. Ideally, passengers using the services of an airport would like to eliminate unnecessary walking distances, minimize waiting times at check-in and baggage claim facilities, and never miss a connecting flight. Moreover, they would like to be able to find their way around the airport easily and to have the quality and prices of concession area goods to be competitive with the outside world.

Configurations which perform well in one dimension (walking distances, for example) may perform poorly in others (congestion and/or baggage transfer reliability). Therefore, the role of the airport designer is to try and achieve a balance among often conflicting measures of performance when trying to select an initial configuration. Such a balance is best achieved when performance is considered in a multidimensional context. As seen in previous examples, however, performance is often described in terms only one or two, rather than several, measures (e.g. the maximum walking distance).

\section{5 - Recommendation for Change}

Figure 1.4 summarizes in matrix form the direction of change that can aid current practice in the decision making process of selecting an initial configuration of airport passenger buildings. The horizontal axis represents the number of dimensions that should be considered when trying to assess the 
potential performance of a particular configuration. The vertical axis represents the range of forecasts that should be considered for each individual measure of performance.

The figure suggests that a single (or few) measure, single forecast approach is prevalent in current practice today, and is represented by the view of the airport building as a "terminal". The arrow points toward the good design practice of considering "broad range, multiple criteria performance", and is represented by the view of the airport building more generally as a "passenger building". Although some design firms may already embrace this idea, it should be clear from the preceding examples that such a philosophy is not yet widespread.

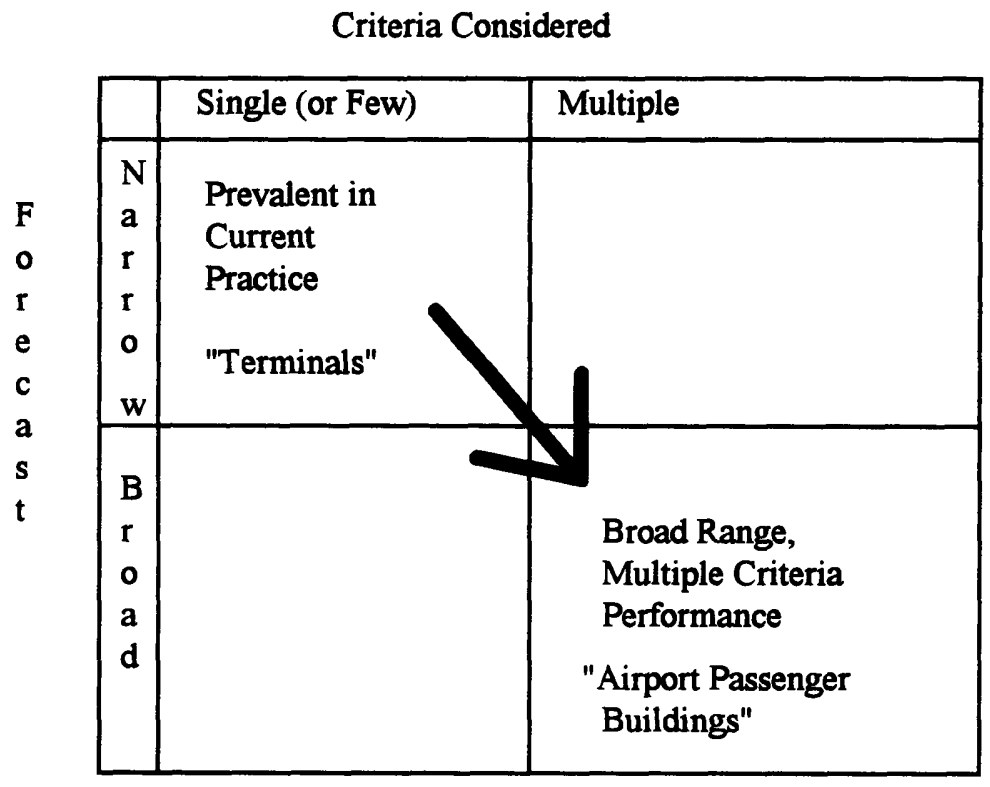

Figure 1.4 : Position Matrix 


\section{6 - Problem Definition and Structure of Thesis}

This thesis : (1) develops a decision support methodology to help airport planners adopt the good design practice of considering performance in a broad range, multiple criteria context, and (2) applies it, as a demonstration of the usefulness of the approach, to the selection of the initial configuration of airport passenger buildings. The methodology is introduced in the form of a series of tools that can quickly evaluate the approximate performance of different passenger building configurations, in order to determine which would be most appropriate, or robust, over a range of conditions. The methodology is intended to be sufficiently general that its efficacy does not rely on specific data requirements; instead, the techniques we present are flexible enough to facilitate as much or as little data as the user can collect.

Chapter 2 reviews the previous work that has been done in the area of decision support for the design of airport passenger building configurations. Research in this area can generally be placed into two different categories; namely, analytic formulae methods and detailed simulation programs. Analytic formulae methods seek to characterize mathematically standard passenger building configurations for various performance measures (such as expected passenger walking distances). Potential performance is described by a single equation in terms of one or more decision variables (e.g. the number and size of individual concourses), and differential calculus and/or other numerical methods are used to find the "optimal" configuration geometry which minimizes the mathematical expression.

On the other hand, detailed Monte-Carlo simulation programs exist which attempt to represent dynamically the airport environment. For landside analyses, individual passengers are "randomly" generated and taken through a series of services based on assumptions of passenger behavior and empirical data regarding average service times at various airport facilities. From such analyses, it is possible to obtain conditional estimates of airport performance (based on assumed conditions) as measured by such metrics as average length and waiting times in queues, average walking distances, aircraft stand utilization, and others.

Chapter 3 traces the evolution of various passenger building configuration concepts, and establishes a basic nomenclature to use when describing specific airport locations. Although airports vary 
significantly both in size and in operational concept, virtually all can be placed into just a few standard categories based on their primary geometry and philosophies of function. Chapter 4 develops geometric abstractions for several of the standard passenger building configurations introduced in Chapter 3 , in order to develop a consistent platform for comparing and contrasting different configuration concepts objectively. Such abstractions are used to obtain the absolute distances between all points within a given configuration which are, in turn, used as inputs to models developed in later chapters.

Chapter 5 develops models for assessing potential configuration performance as measured by issues related to passenger convenience. The models are based on well-known results of geometrical probability, and stress a modular, "object-oriented" approach. We introduce our technique by presenting a general, "spreadsheet" type model to estimate overall passenger walking distances. We then demonstrate how we would incorporate the effects of various operational activities (if such data were available) using the same model. Specifically, we consider the impacts of effective stand management policies (e.g. flightto-gate assignments), varying gate capacities and utilizations, and the dynamic selection of gates during different demand periods. Next we show how we would incorporate the effects of mechanical devices such as moving sidewalks and automated people mover systems in estimating performance measures such as mandatory walking distances and overall passenger travel times. Finally, numerical examples are provided for a sample airport to demonstrate the techniques introduced throughout the chapter.

Chapter 6 uses the geometric representations of the configuration concepts developed in Chapter 3 and applies to them our model for estimating passenger walking distances. The numerical experiments we perform demonstrate how one would select the most appropriate configuration concept and corresponding geometry (number and size of individual concourses) for a given set of data. Results from our experiments are presented in the form of various "performance profiles", which help to demonstrate potential configuration robustness to various input parameters.

Chapter 7 discusses how the general technique for estimating passenger convenience measures can be applied to estimating potential configuration performance as it relates to baggage and aircraft. Although baggage is not "inconvenienced" by long transfer distances like passengers are, minimum baggage connection times must be met in order to avoid delays to aircraft (and thus passengers). We 
discuss the issues most relevant to determining connection times and what data would be needed to estimate them. We then discuss, in a similar fashion, the issues most relevant to "aircraft convenience", as measured by aircraft taxi and apron times.

Chapter 8 presents a model for estimating potential passenger congestion within specific areas of a given building using information already obtained from the models introduced in Chapter 5 . We then show how this model can be used to calculate approximate dimension requirements for a building, based on level of service standards such as those recommended by the International Air Transport Association (IATA). We then present a case study of level of service standards as applied to Boston's Logan International Airport. Finally, Chapter 9 presents our conclusions and discusses directions for future research in this area. 


\title{
Chapter 2
}

\section{Review of Decision Support Methodologies}

\author{
Introduction
}

In order to adopt the good design practice of considering configuration performance in broad range, multiple criteria terms, we need a decision support methodology to help us assess the potential performance of a given configuration. Section 2.1 provides an overview of the decision support methods currently available to airport planners, and suggests where there is a need for a computer-based, planninglevel tool to aid in the selection of the initial configuration of passenger buildings. Section 2.2 discusses in greater detail the decision support offered by several reference manuals and texts on airport planning and design. Section 2.3 reviews the work that has been done on the mathematical characterization of specific measures of airport performance, most notably those pertaining to estimating passenger walking distances. Section 2.4 reviews some of the Monte Carlo simulation programs which planners currently use to help design the detailed layout of airport passenger buildings. Finally, Section 2.5 illustrates how the increasing availability of inexpensive computing power allows us to fill the gap in decision support discussed in the first section. 


\section{1 - Current Methodologies}

As mentioned previously, part of the airport planning process includes the development of the Terminal Area Plan, which includes decisions regarding the configuration of the passenger buildings. The development of a terminal area plan is a two-stage process, beginning with the initial selection of a configuration concept and proceeding with the development of a detailed floor layout. Figure 2.1 outlines the basic two-step process of developing an airport terminal layout plan, including the available decision support methods and some of the authors responsible for work in these areas. Readers interested in an extended bibliography of airport passenger terminal operations analysis and modeling should refer to Tosic [1992].

\begin{tabular}{|c|c|c|c|}
\hline $\begin{array}{l}\text { Terminal Area } \\
\text { Plan Step }\end{array}$ & $\begin{array}{l}\text { Reference } \\
\text { Manuals/Texts }\end{array}$ & $\begin{array}{l}\text { Analytic } \\
\text { Formulae }\end{array}$ & $\begin{array}{l}\text { Computer- } \\
\text { Based }\end{array}$ \\
\hline $\begin{array}{l}\text { Step } 1 \\
\text { Selection } \\
\text { Of Initial } \\
\text { Configuration }\end{array}$ & $\begin{array}{l}\text { IATA, ICAO, FAA, TRB } \\
\text { Parsons Report, } \\
\text { Transport Canada, } \\
\text { Ashford, Blankenship, } \\
\text { Blow, de Neufville, Hart, } \\
\text { Horonjeff and McKelvey }\end{array}$ & $\begin{array}{l}\text { Bandara, } \\
\text { Robuste, } \\
\text { Vandebona, } \\
\text { Wirasinghe }\end{array}$ & Need \\
\hline $\begin{array}{l}\text { Step } 2 \\
\text { Detailed } \\
\text { Layout of } \\
\text { Floor Plan }\end{array}$ & $\begin{array}{l}\text { IATA, ICAO, FAA, TRB } \\
\text { Parsons Report, } \\
\text { Transport Canada, } \\
\text { Ashford, Blankenship, } \\
\text { Blow, de Neufville, Hart, } \\
\text { Horonjeff and McKelvey }\end{array}$ & Impractical & $\begin{array}{l}\text { Dunlay, Pararas, } \\
\text { BAA, FAA, } \\
\text { Transport Canada, } \\
\text { Private Industry }\end{array}$ \\
\hline
\end{tabular}

Figure 2.1 : Available Decision Support Methodologies 


\subsection{1 - Step One : Selection of Initial Configuration and Geometry}

The first step in the development of the airport terminal layout plan is the selection of the initial configuration concept, along with the most appropriate geometry for that concept (number and size of individual departure concourses). Two forms of decision support currently exist for Step One; namely, reference manuals and texts, and analytic techniques.

Reference manuals and texts provide airport planners with very broad information regarding the selection of the initial configuration concept. In general, they contain a discussion of the advantages and disadvantages of different configurations concepts, although they do not provide quantitative evidence to support their statements. Further, the descriptions they provide are generally based on a single, often unspecified set of conditions, with no mention of how their advantages and disadvantages would differ in the face of major change (e.g. becoming a hub, changes in daily demand patterns, etc.). A more detailed description of the decision support offered by several reference manuals and texts is provided in Section 2.2 .

As mentioned previously, analytic formulae techniques characterize potential configuration performance in terms of quantitative measures such as passenger walking distances. Such techniques can appear attractive at the planning level because they are fast and do not require detailed input data. In order to get solutions, however, such "formula" methods impose several unrealistic, simplifying assumptions about the operations and use of the airport passenger buildings. For example, a common assumption made is that aircraft stand utilization is uniform, i.e. that all gates have the capacity to service all sizes of aircraft, and furthermore that the aircraft mix at every gate in the airport is identical [Bandara, Wirasinghe, Robusté].

Such a uniformity assumption, though it makes equations simple to represent, does not characterize what truly occurs -- certain gates are favored for different aircraft based on issues such as maneuverability (e.g. jumbo jets parked at the ends of piers) and passenger convenience (e.g. smaller, less important flights parked further away from central services). Thus, the overall average number of seats which arrive and depart from every gate (over some period of time) is not constant. 
The ability to characterize quantitative performance measures quickly is important to airport designers who must choose among several very different configurations during Step One; however, conclusions drawn from results based on restrictive, often unrealistic assumptions are of little value in the real world. Moreover, the results of such analyses tend to come in the form of a single number, namely the optimal value of the decision variable which minimizes the characterizing equation. For example, consider the following description of selecting the "optimal" geometry for a given passenger building configuration, taken from "Walking Distance Minimization for Airport Terminal Configurations" by Bandara and Wirasinghe [1992b]:

For a centralized satellite terminal the mean walking distance of a passenger can be expressed as a function of the number of satellites only (if the constraint regarding the minimum clearance requirement between the satellites and the terminal block is neglected) and it is possible to choose the optimum number of satellites, $N^{*}$, which will minimize the mean total walking distance.

Little additional information is readily available as to how the configuration would perform under the actual or any other loads. New expressions can be derived and new optimal values can be found, but each time the answer comes in the form of a single number. Extensive sensitivity analyses are difficult when making comparisons using only numerical values, especially when those values vary significantly among the different cases tested. Consider the numerical representation of a sensitivity analysis from Wirasinghe, Bandara, and Vandebona [1987] for a particular type of pier configuration shown in Figure 2.2.

The so-called "optimal" number of concourses varies significantly (even for a given value of $G$ ), with no further clarification of the behavior for the walking distance formula as a function of $N$. Moreover, the "optimal" values they claim to achieve are still based on an oversimplified representation of the problem. Section 2.3 reviews the work that has been done in this area, and summarizes the most common assumptions that are made in order to simplify the characterization of configuration performance. 


$$
\begin{array}{|c|c|}
\hline \multicolumn{2}{c}{G=25} \\
\hline p=0.1 & p=0.4 \\
\hline N^{*}=7 & N^{*}=4 \\
\hline
\end{array}
$$

\begin{tabular}{|l|l|}
\hline \multicolumn{2}{|c}{$G=50$} \\
\hline$p=0.1$ & $p=0.4$ \\
\hline$N^{*}=10$ & $N^{*}=6$ \\
\hline
\end{tabular}

\begin{tabular}{|c|c|}
\multicolumn{2}{c}{$G=75$} \\
\hline$p=0.1$ & $p=0.4$ \\
\hline$N^{*}=13$ & $N^{*}=7$ \\
\hline
\end{tabular}

where

$\mathrm{G}=$ Total number of gates at the airport

$\mathrm{p}=$ Proportion of transfer traffic

$\mathrm{N}^{*}=$ "Optimal" number of individual concourses to minimize walking distances

Figure 2.2: Sensitivity Analyses through Analytic Techniques 1

\subsection{2 - Step Two : Detailed Layout of Floor Plan}

The second step in the development of an airport passenger building layout plan is the creation of the detailed layout of the landside terminal buildings. Decisions such as general space requirements, the number of check-in counters needed and the arrangement/location of security checkpoints are all made during the development of the detailed floor plan. Again, reference manuals and texts provide basic guidelines for terminal layout decisions, generally through a series of charts, figures, and actual layout examples. Such charts and figures, however, often do not capture the dynamic nature of passenger flows throughout the different areas of the passenger buildings. Analytic techniques, such as those developed for Step One, are impractical for Step Two given the level of detail required to provide adequate decision support for layout design. Thus, in addition to manuals and texts, a number of computer-based simulation programs have been implemented to aid airport planners with dynamic information for creating the detailed layouts of passenger buildings.

\footnotetext{
1Source: [Wirasinghe et al. 1987], p.498.
} 
Detailed, Monte-Carlo simulation programs exist which attempt to represent dynamically the airport environment. For landside analyses, individual passengers are "randomly" generated and taken through a series of services based on assumptions of passenger behavior and empirical data regarding average service times at various airport facilities. These programs generally require sample flight schedules and much detailed information regarding the landside terminal configuration itself. Specifically, a complete layout of the airport is often needed, including for example, the total length of baggage claim belts and the number and arrangement of security checkpoints. Acquiring such input data can be time consuming, however, and is independent of the actual computation time of the simulation program. One estimate for the setup time involved with simulating just one Terminal (E) at Boston Logan International was "at least three weeks", with more time required for data verification.

From such analyses, it is possible to obtain conditional estimates of airport performance (based on assumed conditions) as measured by such metrics as average length and waiting times in queues, average walking distances, aircraft stand utilization, and others. Although these estimates provide information regarding potential configuration performance, the time required to obtain them would be cost prohibitive if more than just a few concepts are to be studied -- making comparisons among many different configuration concepts (using simulation programs) becomes unattractive given the total cost in terms of time. Even as computation times decrease with the advent of more powerful microprocessors, the setup times (expressed in terms of weeks) remain the limiting factor.

Therefore, it is unlikely that such detailed decision support would be practical at high levels of the design process (Step One) given the computationally intensive nature of the problem solving methodology. Rather, computer-based simulation programs are appropriate only after most or all of the inappropriate design alternatives have been eliminated. It is possible, for example, that two configuration alternatives demonstrate similar performance characteristics after analyses are performed in Step One. In order to choose between the two specifically defined configuration concepts, the use of computer simulations may elicit precise information relevant to the decision making process at that stage; however,

${ }^{2}$ Based on conversation with Robert Shumsky, summer analyst at Massport in charge of implementation. 
for the selection of the initial configuration concept, reference manuals and analytic formulae remain the only means of decision support.

\subsection{3 - Filling the Gap}

Absent from the overall design process is a computer-based decision support methodology that can quickly evaluate the approximate performance of different passenger building configurations, in order to determine which would be the most appropriate over a range of assumptions and a variety of performance measures (i.e. broad range, multiple criteria performance) [Odoni and de Neufville 1992]. As mentioned previously, it is the intent of this thesis to develop a series of tools to aid airport planners in selecting the initial configuration of passenger buildings. We will refer to this set of tools more generally as a Passenger Building Configuration Evaluator, or PBCE.

The PBCE tools we develop take advantage of available computer technology in performing "back-of-the-envelope" type calculations, based on the amount of data available to the user. We will demonstrate that even with limited information, it is possible to estimate potential configuration performance so that comparisons among different concepts can be made. We will also show how as more information becomes available, it can be incorporated into the basic PBCE "shell" without having to alter the general methodology. This feature allows for greater flexibility in performance estimation than is available through the analytic techniques described previously.

The methodology we present is sufficiently general that it can be used to estimate several different measures of potential configuration performance, over a range of different possible conditions. Specifically, we will examine measures related to passenger convenience, baggage connection reliability, and aircraft taxi times; however, many other performance measures exist [Lemer 1990]. The models we develop are intended to demonstrate a general decision support technique, rather than to produce an exhaustive study of potential airport performance. 


\section{2 - Reference Manuals and Texts}

The most basic forms of decision support used to aid airport planners in the design of passenger buildings are reference manuals and texts. These sources provide general information on the overall airport planning process itself, as well as standards developed by regulatory groups such as the FAA, IATA, ICAO, and others. Depending on the level of detail included, reference manuals and texts can be used for both steps in the development of the Terminal Area Plan; namely, the selection of the initial configuration and the planning of the detailed floor layout.

\subsection{1 - Step One : Selection of Initial Configuration}

Also known as the "concept development" stage [Horonjeff and McKelvey 1983], the first decision in the selection of the initial configuration of passenger buildings frequently involves the fundamental choice of whether passenger processing facilities will be centralized or decentralized. According to Ashford and Wright [1979],

with centralized concepts, all the elements in the passenger processing sequence are conducted as far as feasible in one localized area. Processes normally included are ticketing, check-in, customs and immigration, baggage checking and claim, and possibly security. All concession and ancillary facilities are also grouped in the central terminal area. Decentralization involves a spreading of these functions over a number of centers in the terminal complex; the concept embraces the range of possibilities from using independent terminals for various airlines (the unit terminal concept) to simply providing facilities at the aircraft for the lightly loaded traveler to perform a a complete check-in (the gate check-in concept) In practice many design solutions fall between the extremes of completely centralized and decentralized operation. [p.240]

Once the choice is made as to the overall operational philosophy of the passenger processing facilities (i.e. centralized or decentralized), the second decision in Step One pertains to the physical arrangement of the departure/arrival concourses, the interface between passengers and aircraft. Reference manuals and texts generally recognize that most airport configurations can be placed into one of three (or four) basic concepts, as well as variations or "hybrids", which combine the features of two or more of the 

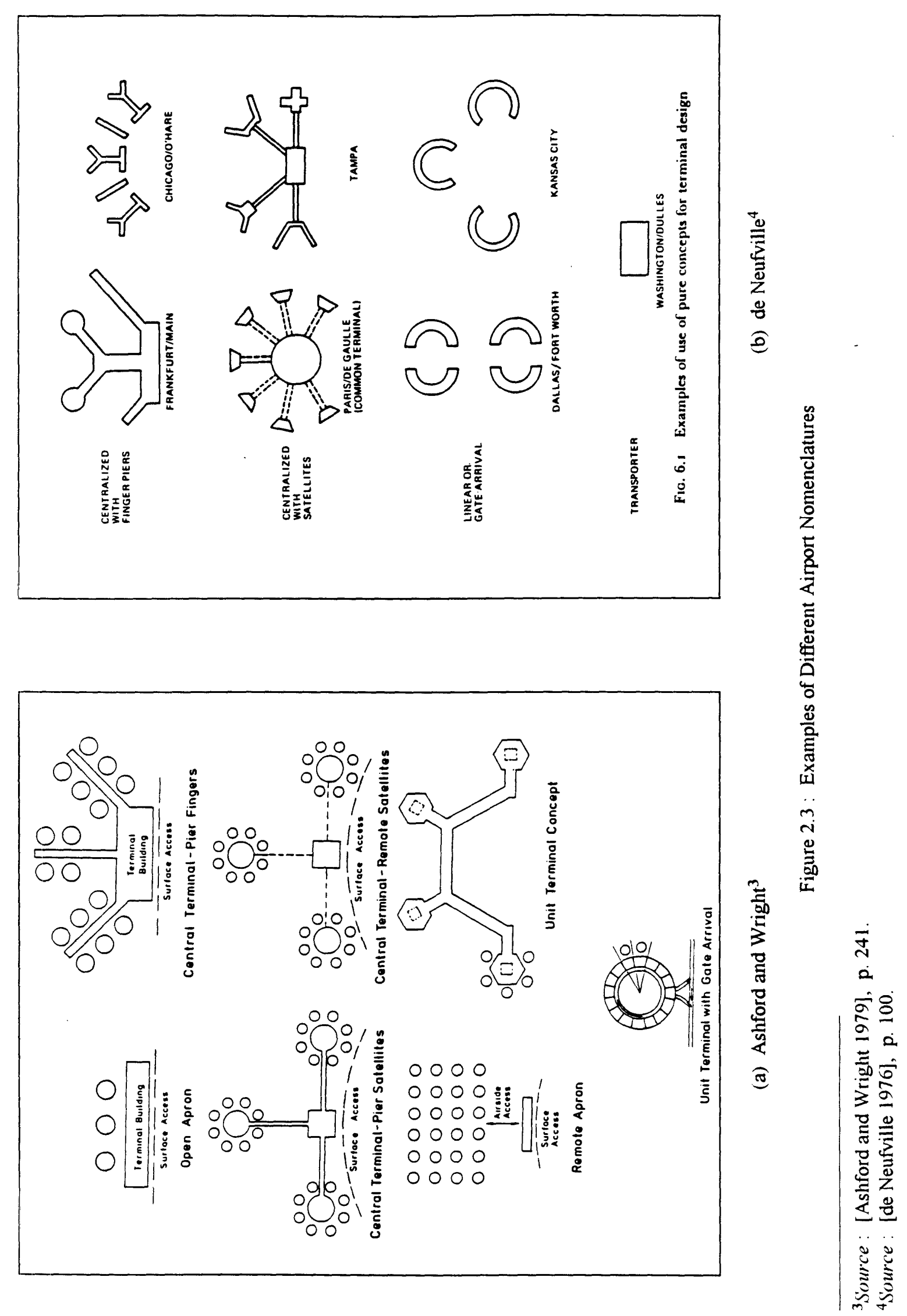
basic concepts. Figure 2.3 illustrates the basic airport nomenclature adopted by different authors of airport planning texts. These concepts are described in greater detail in Chapter 3.

Although useful in providing planners with a standard taxonomy for discussing different configuration concepts, both qualitative and quantitative comparisons among different types are rare.

Figure 2.4 is taken from "Section 3: The Passenger Terminal" of the Airport Terminals Reference

Manual [IATA 1989], and describes the major advantages and disadvantages of a centralized pier/finger concept.

\section{EXAMPLE OF PIER/FINGER CONCEPT}

Centralized Terminal

Major Advantages

Centralization of airline and

Government Authority processing

personnel.

Permits centralization of terminal facilities/amenities (i.e. restaurants, duty-free, etc.)

Permits use of relatively simple flight information display systems.

Facilities control of passengers, if required.

\section{Major Disadvantages \\ Long walking distances.}

Kerbside congestion in peak hours.

Limited expansion capability of main terminal due to the complex building geometry.

Reduced aircraft circulation \& maneuverability; limited compatibility with future larger aircraft design development.

Separation of arriving/departing passengers, if required, must be by different levels ( 3 level finger).

Early check-in and close-out times.

High capital, operating and maintenance costs for passenger moving \& baggage conveying/sorting systems; potential for baggage mishandling.

Figure 2.4 : Decision Support for Initial Configuration Selection ${ }^{5}$

5Source : [IATA 1989] 
The description is useful only in a very general context, however, one in which the merits and shortcomings of different configurations are compared using only a superficial analysis. For example, the identification of "early check-in" and "long walking distances" as major disadvantages relates to the fact that the aircraft stands are separated from the centralized passenger processing facilities (located in the main terminal building). Thus, relative to a gate arrival concept, for example, passengers must arrive at the check-in area earlier and traverse longer distances in order to reach their departure gate.

Such a qualitative treatment ignores the issues of how much longer walking distances are (and relative to what) and how much earlier passengers must check-in. Moreover, the description is pertinent only for originating passengers and does not consider the potential reduction in walking distances for transfer passengers. It is unlikely that a thorough understanding of the details of the selection process (Step One) can be obtained from such a cursory description.

\subsection{2 - Step Two : Detailed Layout of Floor Plan}

Once the configuration of passenger buildings has been selected, the development of the Terminal Area Plan can proceed with Step Two, the detailed layout of the floor plan. Specifically, space requirements for individual facilities need to be determined based on the anticipated (present and future) airport demand. According to Ashford and Wright [1979],

To assure orderly and smooth functioning of the terminal, the individual facility areas that form the constituent parts should be designed to accommodate the level and type of passenger loading they are expected to experience. This process ideally requires the following steps :

- Determination of peak hour design demand.

- Statement of passenger traffic by type.

- Identification of individual facility volumes.

- Calculations of space requirements.

Reference manuals and texts provide a number of charts and figures to aid airport planners with space requirements for individual facilities. Some examples are shown in Tables 2.1 and 2.2 and Figures 2.5 and 2.6. Note that the space requirements in Table 2.2 are based on the TPHP (typical peak hour 
passenger) design parameter, used by the FAA. The TPHP is an estimate of peak (hourly) demand that will be exceeded only for short periods of time. [Ashford and Wright 1979].

Although useful for gross space approximations, the figures and charts provided by reference manuals and texts frequently neglect certain key issues when determining facility requirements. Figure 2.5, for example, provides general guidelines for the allocation of terminal area space. These guidelines are not site-specific, however, and thus do not take into consideration characteristics relevant to individual airports. For example, the allocation of waiting areas ( 30 percent of the space designated for non-rentable use) and for concessions ( 17 percent of the space designated for rentable use) does not consider the mix of passengers anticipated at the airport, a significant determinant of which terminal area facilities are most frequently used. For example, international passengers typically have to check-in earlier than local passengers, and thus require more shops and restaurants.

The chart in Figure 2.6 is meant to help determine the number of seats required in the waiting lobby area, based on a floor space approximation such as the one illustrated in Figure 2.5. Again, however, no mention is given as to the type of traffic expected (international vs. domestic, originating/terminating vs. transfer, etc.). As such reference manuals and texts provide decision support only at the most preliminary stages of the floor layout analysis, and are desirable more for their speed and ease of use than for their flexibility.

Table 2.1 : FAA Recommended Relationships for TPHP Computations from Annual Figures ${ }^{6}$

\begin{tabular}{cc}
\hline $\begin{array}{c}\text { Total Annual } \\
\text { Passengers }\end{array}$ & $\begin{array}{c}\text { TPHP as a Percentage } \\
\text { of Annual Flows }\end{array}$ \\
\hline million and over & 0.030 \\
$10,000,000-19,999,999$ & 0.035 \\
$1,000,000-9,999,999$ & 0.040 \\
$500,000-999,999$ & 0.050 \\
$100,000-499,999$ & 0.065 \\
under 100,000 & 0.120 \\
\hline
\end{tabular}

6Source : [FAA 1969] 
Table 2.2: FAA Space Standards 7

Domestic Terminal

Space Requirements per 100 TPHP,

Space Facility

$\left(1000 \mathrm{ft}^{2}\right.$ or $100 \mathrm{~m}^{2}$ in each facility

Ticket Lobby

Airline operational

1.0

Baggage claim

4.8

Waiting rooms

1.0

Eating facilities

1.8

Kitchen and Storage

1.6

Other Concessions

1.6

Toilets

0.5

Circulation, mechanical and

maintenance, walls

$0: 3$

$\underline{11.6}$

Total

24.2

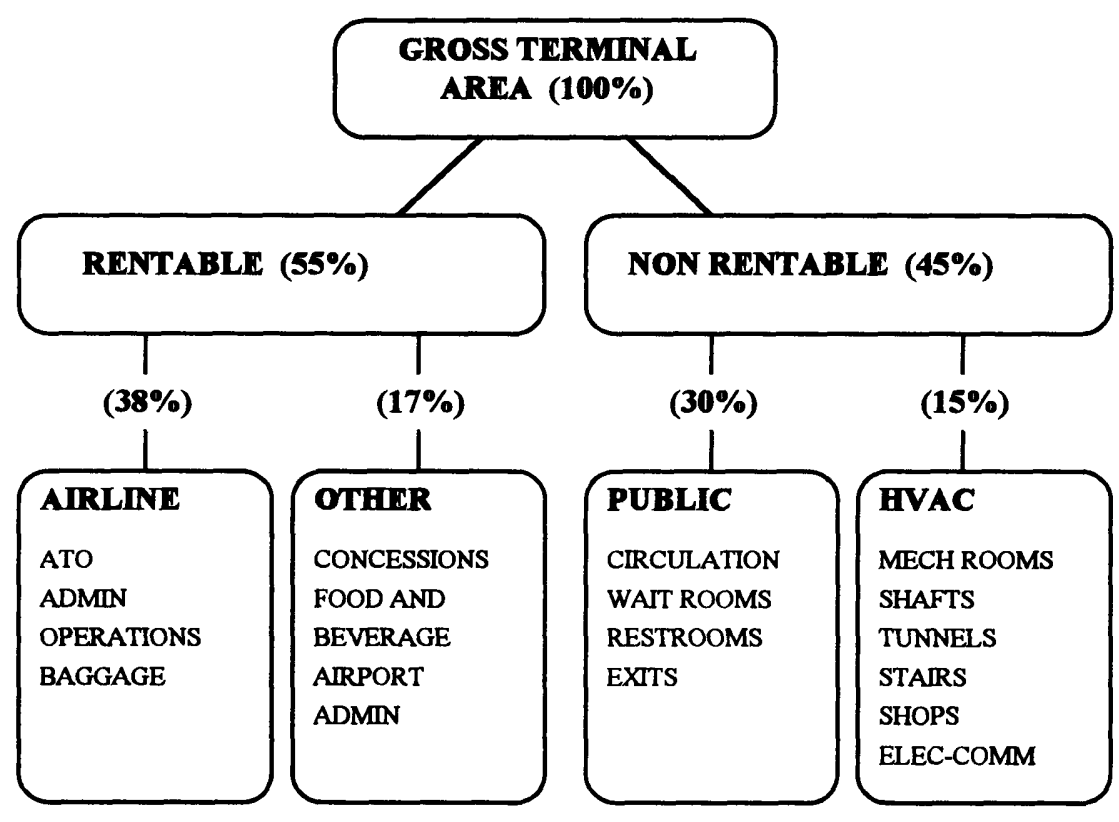

Figure 2.5 : Decision Support for Detailed Floor Plan Layout ${ }^{8}$

${ }^{7}$ Ibid.

${ }^{8}$ Source : [Parsons 1975], p. 6-9. 


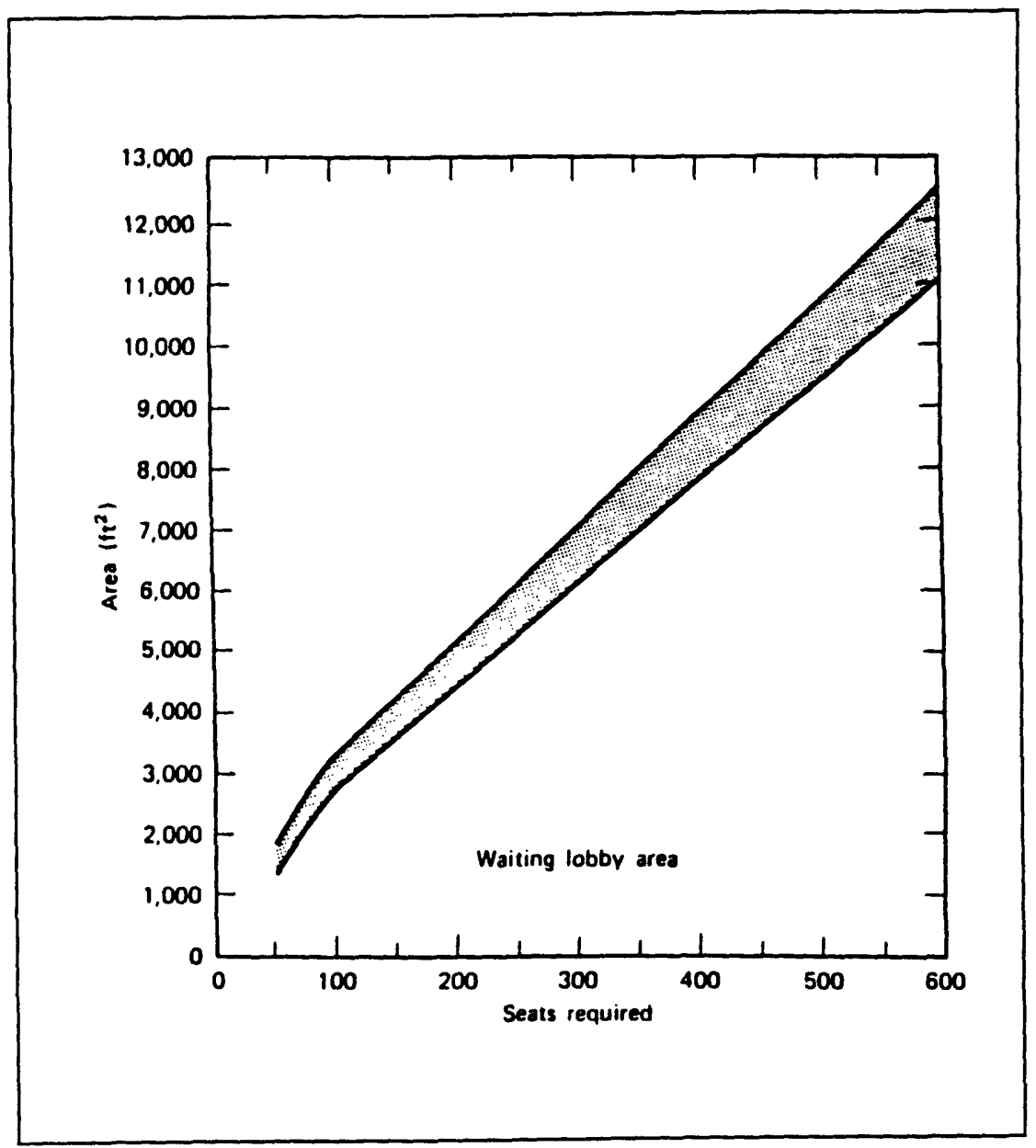

Figure 2.6 : Decision Support for Waiting Area Sizing ${ }^{9}$

${ }^{9}$ Ibid., p. 4-12 


\section{3 - Analytic Formulae Techniques}

The following section discusses several analytic techniques that have been developed as a means of decision support for the selection of the initial configuration of passenger buildings. It includes a detailed description of techniques used to estimate measures related to passenger convenience (as measured by overall walking distances), and methods used to determine the gate position requirement at an airport. Finally, other measures such as the seating capacity of departure lounges and the optimal rate of customs inspection are discussed.

\subsection{1 - Passenger Walking Distances}

Early research in quantitative decision support for the minimization of passenger walking distances concentrated on improving the assignment of a schedule of flights-to-gates for a fixed configuration. Braaksma [1977] showed that methods for improving the gate assignment problem could reduce passenger walking distances dramatically. The formal gate assignment problem was formulated by Babic et. al. [1984] who proposed a branch-and-bound algorithm for solving the large combinatorial problem of matching arriving aircraft with aircraft stands. Their formulation considered only originating and terminating passengers, however, and did not take the walking distances of transfer passengers into account.

Mangoubi and Mathaisel [1985] incorporated transfer passengers into their formulation of the flight-to-gate assignment problem. Their integer programming formulation to minimize the total distance, $Z$, walked by all passengers is expressed as :

$$
\operatorname{Min} Z=\sum_{i=1}^{M} \sum_{j=1}^{N}\left(p_{i}^{a} d_{j}^{a}+p_{i}^{d} d_{j}^{d}+p_{i}^{t} d_{j}^{t}\right) x_{i j}
$$


where the estimated number and of arriving, departing, and transferring passengers using flight $i$ are denoted $p_{i}^{a}, p_{i}^{d}$, and $p_{i}^{t}$, respectively. Similarly, the walking distances for passengers using gate $j$ are $d_{j}^{a}, d_{j}^{d}$, and $d_{j}^{t}$. A binary variable, $x_{i j}$, is defined for each possible flight-to-gate assignment such that :

$$
x_{i j}=\left\{\begin{array}{l}
1 \text { if flight } \mathrm{i} \text { is assigned to gate } \mathrm{j} \\
0 \text { otherwise }
\end{array}\right.
$$

The authors also offer suggestions on how to modify the original formulation to include solutions to common objections of an airport-wide assignment. Issues as how to deal with subdividing the airport into separate airline areas, excluding certain aircraft types from certain gates, and requiring that certain flights be assigned to nearby gates are discussed. In addition to the (integer) linear program formulation, a heuristic algorithm is also described and computational results are given which demonstrate that the use of either method in test cases can reduce walking distances by over 30 percent. The heuristic method was also shown to come within an average of 3.9 percent of the optimal in many cases.

Both the preceding approaches for minimizing passenger walking distances assume that a specific configuration is given and thus that all walking distances are known and fixed. Such models are appropriate for tactical approaches to reducing passenger walking distances at existing sites; however, from a strategic perspective, the fact that the configuration is already selected (and that an entire flight schedule is required) limits the effectiveness of such formulations for long term planning. It is unreasonable, for example, to assume that a current flight schedule will accurately reflect conditions ten or even twenty years hence. Moreover, traffic volumes and the passenger mix at an airport may change significantly over time, thus altering the estimated values for parameters used as inputs.

Decision support for minimizing passenger walking distances from a long-term planning perspective has concentrated on geometrically characterizing certain standard terminal configurations and deriving explicit equations to describe walking distances for different passenger types. Wirasinghe and Vandebona [1987] studied walking distance distributions in single- and dual-concourse centralized airport terminals. Studying what they referred to as "quasi-linear" configurations (those containing a centralized 
terminal block and aircraft gates located on both sides of attached, extended concourses), the authors used simulation techniques to derive the cumulative walking distance distributions for three passenger classifications : hub transfers, normal transfers, and arrivals and departures (a single classification). Hub transfers, also referred to as direct transfers, are assumed to be preticketed and thus walk directly from their arrival gate to their departure gate. "Normal" transfers, more commonly known as indirect transfers, are connecting passengers who transfer from one aircraft to another, but who have to be reticketed at the terminal block.

For the purposes of their simulation, the authors employed several simplifying assumptions, specifically : that all gates are capable of handling all aircraft types, and passengers are equally likely to arrive and depart from any gate, i.e. that gate utilizations are identical. They do acknowledge that airlines can reduce walking distances by judiciously assigning flights-to-gates; however, their simulation does not account for such actions. From their examination of four centralized configuration types (singleconcourse, basic dual-concourse, T-shaped dual-concourse, and rectangular dual-concourse), the authors concluded that a T-shaped configuration provides the best level of service from the objective of minimizing the fraction of passengers who must walk a distance greater than $350 \mathrm{~m}$. Also, they report that the $\mathrm{T}$-shaped configuration is suitable for most fractions of transfers, but that a single-concourse can minimize walking if all passengers are hub transfers.

Wirasinghe, Bandara, and Vandebona [1987] also characterized different configuration geometries, this time using mathematical equations rather than simulation-based techniques. In their paper, the authors investigated finger-pier type buildings in order to facilitate the selection of a specific configuration concept and geometry (number and size of concourses) during the planning stage. Three primary types of finger-pier configurations were studied : centralized-radial, centralized standard, and semi-centralized. Again, an identical gate assumption was employed, along with uniform gate spacing and utilization. Expressions were derived for the walking distances of originating/terminating and indirect transfer passengers (direct transfers were not considered), in terms of the number of concourses and several constants used to describe the size of fixed terminal elements (e.g. the terminal block, spacing between gates and between concourses, etc.). Using differential calculus and other numerical methods, 
the optimum geometries for each of the three types of configurations were found based on different assumptions of passenger mix.

Based on the optimal geometries found, various performance characteristics were studied including the means and variances of overall passenger walking distances, the mean excess walking distance (distance walked above some threshold), and the probability of walking a distance greater than some threshold. The authors concluded that all three configurations could be characterized such that optimal geometries could be found, and that the optimal geometries for both centralized concepts were independent of the fraction of transfers. Further, they found that the optimal number of piers (concourses) for the standard-centralized and semi-centralized configurations were nearly proportional to the square root of the number of gates at the airport.

Bandara applied a similar analytic characterization of terminal geometries to satellite-type airports $[1989,1990]$. In his papers he identified the primary types of satellite concourses (circular, rectangular, Y-shaped, and T-shaped), all of which can be arranged in centralized or semi-centralized concepts. He defined the space allocation for apron facilities in terms of the angle subtended by the intersection point of the outermost connector centerlines, denoted by $\beta$. Centralized satellite configurations are characterized by equal spaced concourses arranged radially about a single terminal block (Figure 2.7a). Semi-centralized satellite configurations are characterized by equivalently shaped (not necessarily equally sized) concourses, each with an individual terminal block, all arranged in a semicircular fashion (Figure 2.7b). The author provides an informal explanation why non-uniform satellites can reduce walking distances for transfer passengers (similar to the previous argument regarding piers), but again other considerations are likely to play a more important role in sizing of individual satellites.

For his analysis, Bandara derived expressions for three categories of passengers: non-hub (indirect) transfers, hub (direct) transfers, and arriving and departing (originating and terminating) passengers. He concluded that, in general, the optimal number of satellites for a given configuration is governed by the length of the connectors between the concourses and the terminal blocks, and thus that the angle of spread, $\beta$, heavily influences the optimal geometry. In addition, he concludes that centralized concepts outperform semi-centralized concepts when the percentage of hub (direct) transfers is high. 
Similar to pier-type airports, the lower and upper bounds for the optimal number of satellites were found to be proportional to the square root of the total number of gates at the airport.

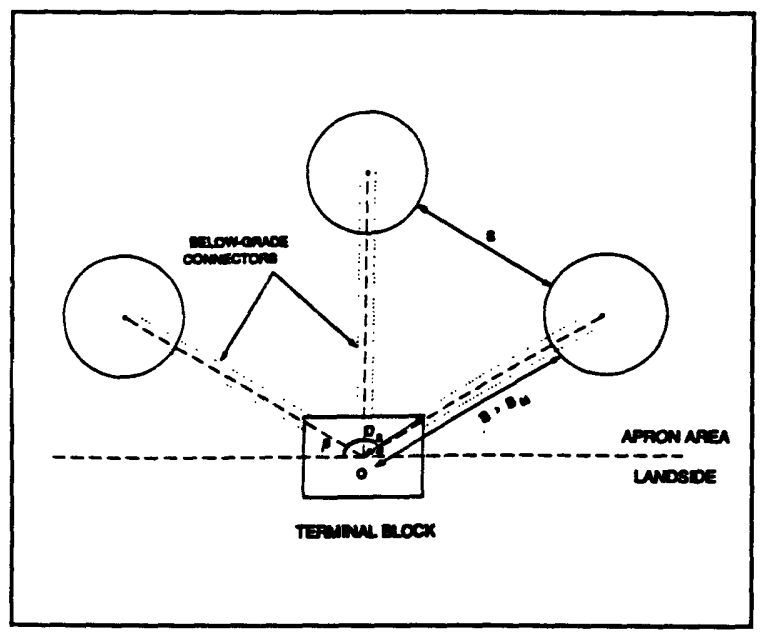

(a)

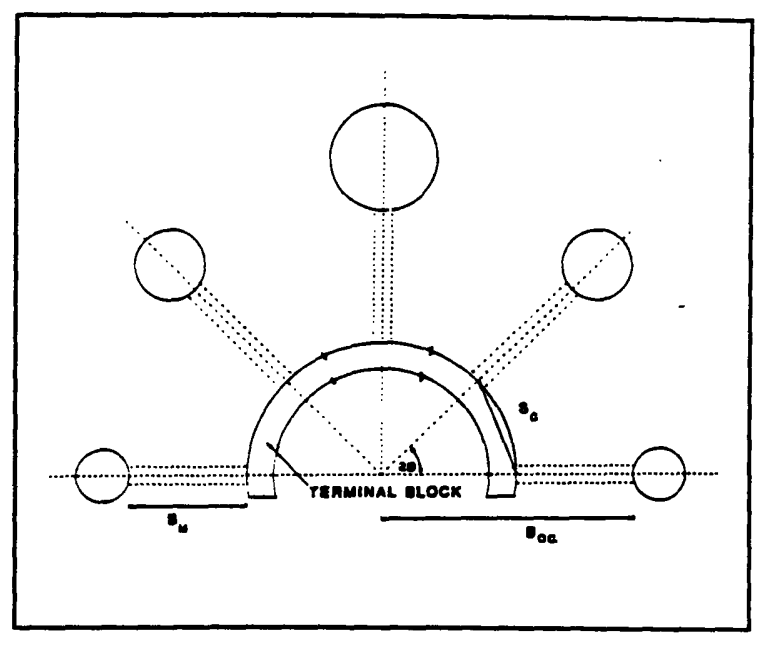

(b)

Figure 2.7 : Centralized and Decentralized Satellite Characterizations ${ }^{10}$

Robusté [1991a] characterized configurations having single, open concourses, closed-loop concourses, inverted closed-loop concourses, and multiple piers. For each configuration he derived expressions for the expected distance walked by originating and terminating passengers, denoted by $D_{0}$, and the distance walked by transfer passengers, denoted by $D_{1}$. If the proportion of transfer passengers is denoted by $\tau$, then the average distance walked by all passengers is simply

$$
D_{\tau}=(1-\tau) D_{0}+\tau D_{1}
$$

For his analysis, Robusté assumes that the airport has a fixed number of gates, $G$, evenly spaced by a distance $g$ and all able to accommodate any aircraft type. Further, he assumes the generic passenger has an equal chance of having to walk to any of the gates, and that the entire terminal configuration has "axial symmetry" about a line perpendicular to the terminal block.

\footnotetext{
${ }^{10}$ Source: [Bandara and Wirasinghe 1992b], p. 62-63.
} 
Based on certain assumptions about the dimensions of the different terminal components, Robusté compared average walking distances for various values of $\tau$. He confirmed the common belief among airport planners that, in general, (1) closed-loop concepts (Figure 2.8) are insensitive to the proportion of transfers, (2) linear concourses, radial piers, and parallel piers favor originating and terminating passengers, and that (3) a single pier (with gates on both sides) favors transfer passengers.

Bandara and Wirasinghe [1992a,b] extended their work on pier and satellite-type terminal configurations to include hub or direct transfer passengers in their estimate of overall walking distances. The addition of hub transfer passengers required the addition of two new input parameters into their walking distance models: the proportion of transfer traffic that consisted of hub transfers, $Q$, and the fraction of hub transfers who depart from their arrival concourse, $r$. The authors recognized that due to the "ownership" of terminal areas in the U.S. (brought about by long-term leases with the airlines) the likelihood that an arriving passenger will depart from the same concourse is potentially much higher than that passenger departing from other concourses. Therefore, they calculate separate estimates for passengers who remain in their departure concourse and those who are equally likely to depart from any gate in the airport.

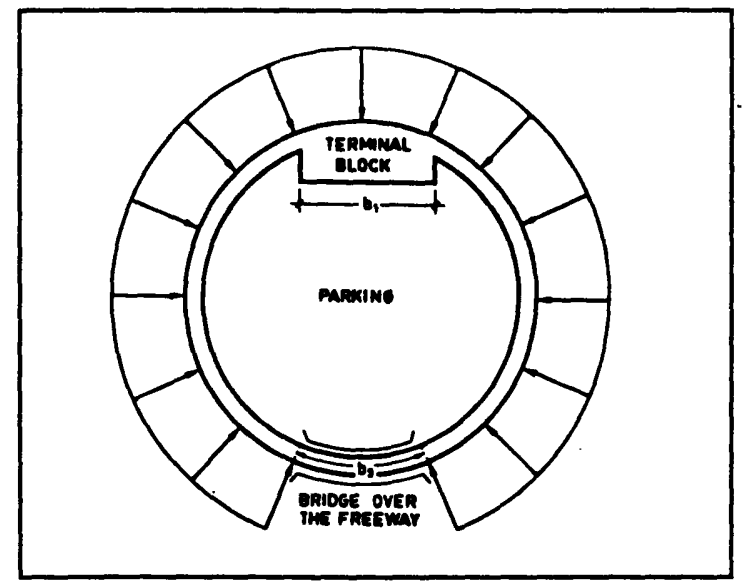

Figure 2.8 : Closed-Loop Centralized Hub Terminal ${ }^{11}$

\footnotetext{
${ }^{11}$ Source: [Robusté 1991a], p. 148.
} 
Their inclusion of hub transfer passengers into the expression for the overall walking distance estimate also added the possibility that unequal concourse sizes will provide an "optimal" geometry when trying to minimize the walking distance expressions. Due to the structure of the characterizing equations used to describe centralized parallel pier terminals, it is possible to reduce the walking distances of a uniform concourse geometry by making the concourses closer to the terminal block longer than those farther away. Such a characterization, however, does not consider that smaller piers farther away from the terminal block are likely to be much less desirable to airlines than the longer, more conveniently located ones. For this reason, it is unlikely that a non-uniform geometry would ever be constructed based on the ability to minimize walking distances alone.

From their analysis of pier-type terminal configurations, the authors were able to derive expressions for the lower and upper bounds on the number of concourses which minimize overall passenger walking distances (no closed-form solutions were found). These equations can be solved using various analytic techniques, and the actual "optimal" geometry can be found by comparing walking distance estimates for geometries between the two extremes. Using such techniques they found that centralized radial and centralized parallel pier terminals are less sensitive to changes in passenger mix, but that the actual mean walking distance estimates for semi-centralized terminals (of the same size) were lower under many circumstances.

Wirasinghe and Bandara [1992] also examined the planning of parallel pier airport passenger buildings which employ automated people mover (APM) systems for transit between piers. Such airports as Atlanta's Hartsfield and the New Denver Airport, also referred to as midfield concepts, both use APM systems for moving originating and terminating passengers from the terminal block to concourses midfield, as well moving transfer passengers between concourses (Figure 2.9). An expression for the total disutility of the system (passengers' disutility of walking, disutility of using the APM, and the associated costs of the APM) is derived in terms of a number of input parameters (passenger mix, gate spacing, etc.) and decision variables (number and size of piers). The expression is then minimized using numerical techniques, and the optimal geometry which minimizes the total disutility of the system is determined. 
When no closed form solution exists, lower and upper bounds are derived and the optimal geometry is determined by other numerical methods.

Wirasinghe and Bandara also explore the sensitivity of the utility expression to the various input parameters used such as user costs (value of time), operator costs (capital and maintenance expenditures), terminal size and passenger mix. The authors found that the geometry which minimized total system disutility consisted of a non-uniform set of piers with longer piers toward the terminal block. When the percentage of hub transfer passengers is high, however, they found that the optimal geometry tends toward uniform pier lengths. They also found that the optimal geometry is most sensitive to the ratio of the disutility of walking to the disutility of riding the APM system. The range of this ratio tested was between 3.3 and 1 (a value of 3.3 means that the walk cost/unit time $=3.3 \times$ ride cost/unit time), based on earlier studies of choice models for transit within airport terminal buildings employing automated people mover systems [Kumarage and Wirasinghe 1990].

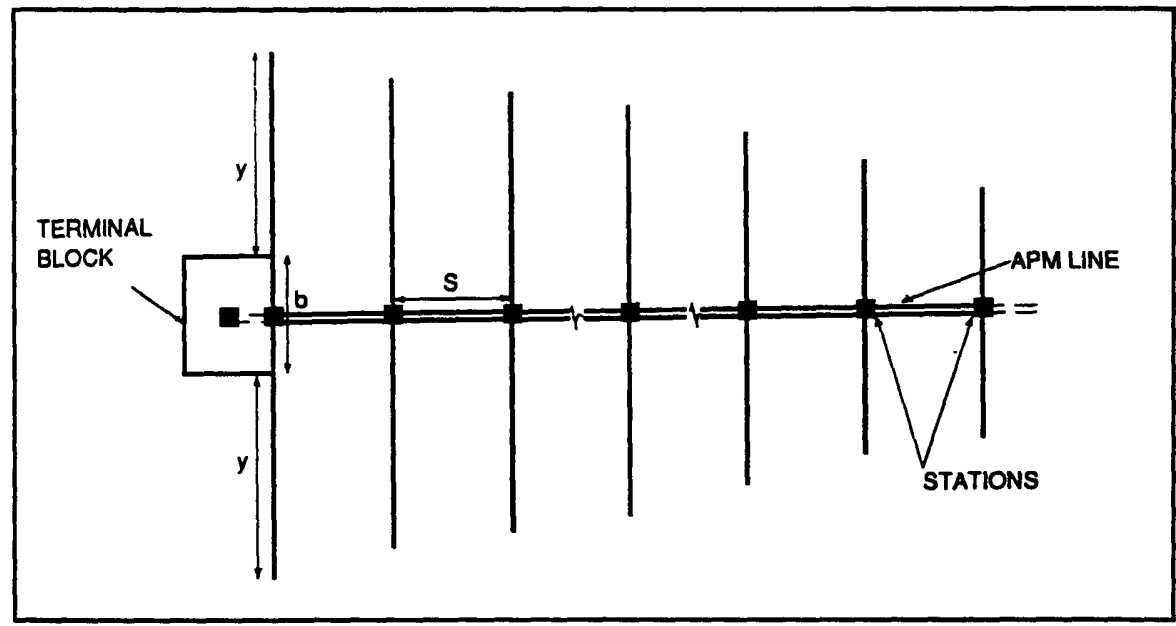

Figure 2.9: Centralized Remote Pier Characterization ${ }^{12}$

Throughout their analyses, Wirasinghe et. al. employ a continuum approximation for walking distances within a passenger building, i.e. rather than enumerate all distances to individual gates within a departure concourse, the authors assume passengers can depart from any point along the entire length of

${ }^{12}$ Source : [Wirasinghe and Bandara 1992], p. 36. 
the concourse [Wirasinghe 1988]. From such an approximation it is possible to use well-known properties of the uniform distribution to estimate walking distances within the concourse. Under a uniform gate assumption, the error associated with employing a continuous approximation for intraterminal walking distances is shown to be small (along the order of 5 percent for hub transfers in a 20gate airport) and this error decreases as the number of gates increases. For walking distances between piers and satellites, however, the error is inversely proportional to the number of piers (which is typically small), and thus discrete distances are still employed. Table 2.3 lists some of the basic elements desirable in an analysis of potential configuration performance, along with the authors responsible for the analytic formulae techniques discussed previously. Noticeable absent from the literature is an attempt to consider situations in which gate size is not fixed and passenger departures are non-uniform.

Table 2.3 : Analytic Studies have Considered Only a Limited Range of Elements

\section{Elements Desirable when Considering Configuration Performance}

\begin{tabular}{|l|c|c|c|c|c|c|}
\hline \multicolumn{1}{|c|}{ Authors, } & Orig- \\
[Year] & $\begin{array}{c}\text { Term } \\
\text { Pax }\end{array}$ & $\begin{array}{c}\text { Hub } \\
\text { Trans } \\
\text { Pax }\end{array}$ & $\begin{array}{c}\text { Non- } \\
\text { Hub } \\
\text { Trans } \\
\text { Pax }\end{array}$ & $\begin{array}{c}\text { Gate } \\
\text { Size } \\
\text { Not } \\
\text { Fixed }\end{array}$ & $\begin{array}{c}\text { Unequal } \\
\text { Pax } \\
\text { Bldg } \\
\text { Size }\end{array}$ & $\begin{array}{c}\text { Non- } \\
\text { Uniform } \\
\text { Pax } \\
\text { Depts }\end{array}$ \\
\hline Wirasinghe and Vandebona [1987] & $\bullet$ & $\bullet$ & $\bullet$ & & & \\
\hline Wirasinghe, et. al. [1987] & $\bullet$ & & $\bullet$ & & & \\
\hline Wirasinghe [1988] & $\bullet$ & $\bullet$ & $\bullet$ & & & \\
\hline Bandara [1989], [1990] & $\bullet$ & $\bullet$ & $\bullet$ & & $\bullet$ & \\
\hline Robusté [1991a] & $\bullet$ & $\bullet$ & & & $\bullet$ & \\
\hline Bandara and Wirasinghe [1992a], [1992b] & $\bullet$ & $\bullet$ & $\bullet$ & & $\bullet$ & \\
\hline Wirasinghe and Bandara [1992] & $\bullet$ & $\bullet$ & $\bullet$ & & $\bullet$ & \\
\hline
\end{tabular}

\subsection{2 - Gate Position Requirements}

In addition to passenger walking distances, analytic methods have also been developed for determining the number of aircraft stands required at an airport for a given traffic forecast. Similar to the walking distance models, the gate position requirement models make simplifying assumptions in order to represent the problem in equation form. Although McKensie et.al. [1974] first addressed this problem 
using simulation techniques, others sought to solve the problem using less computationally intensive means. Deterministic models [Horonjeff 1962] essentially follow the form :

$$
G=A T u
$$

where :

$$
\begin{aligned}
G & =\text { total gate position requirement } \\
A & =\text { average arrival rate of aircraft, } \\
T & =\text { average gate position occupancy time } \\
u & =\text { utilization factor. }
\end{aligned}
$$

The utilization factor, $u$, is crucial to the efficacy of the model, but it is very difficult to estimate.

Empirical data can be used to obtain site-specific estimates, but such data are unlikely to be useful for long-term planning purposes or for use at other sites.

Early stochastic models were proposed by Knowler and Rallis, applying various standard queuing models to the problem of determining the gate position requirement. In both approaches aircraft (customer) arrivals are assumed to follow a Poisson process, and aircraft stands are treated as the "servers". Knowler [1964] used an Erlang loss formula in his model which assumed that if an aircraft arrived when all gate positions were being used, the aircraft simply left the system, rather than queuing until a gate was open, while Rallis [1967] assumed gate occupancy times were exponentially distributed (contrary to empirical evidence). In both approaches, the simplifying assumptions used to create the characterizing equations make results suspect.

Steuart [1974] proposed a probabilistic approach to the problem, which incorporated actual flight schedule data. He used deterministic flight arrival and departure times based on a schedule and a probabilistic component to represent the probability that a flight actually occupies its intended gate at a given time. The model was used to study the effects of different scheduling strategies on aircraft stand requirements, particularly the influence of "banking" flights at a hub. He found that, in general, the more uniform a schedule the fewer gate positions required, and that banking tended to increase the required number of gates. Further, he was able to express the total gate position requirement as the sum of a number of independent random variables, each with a finite variance. Thus by the Central Limit 
Theorem, the average gate requirement he determined plus one standard deviation was sufficient to handle traffic approximately 84 percent of the time, and the average requirement plus two standard deviations was almost certain to handle all traffic for the given schedule.

Bandara and Wirasinghe [1989] later proposed a means of determining the gate position requirement under uncertainty based on an expression equivalent to Equation 2.4. In this case, however, the utilization of gate positions is expressed in terms of an aircraft separation (buffer) time, $S$. The gate position requirement expression then becomes

$$
G=A T\left(\frac{T+S}{T}\right) \quad \text { or } \quad G=A(T+S)
$$

where

$$
\begin{aligned}
& G=\text { number of gate positions required } \\
& A=\text { arrival rate of aircraft } \\
& T=\text { gate occupancy time } \\
& S=\text { the aircraft separation (buffer) time. }
\end{aligned}
$$

By separating the utilization factor into its constituent components, the authors were able to test individual distribution assumptions for the gate occupancy time, $T$, and the aircraft separation time, $S$, in order to perform sensitivity analyses on the estimates for the gate position requirement. Again, although the model for the gate position requirement can be represented by a single equation, values for the input parameters are unlikely to be readily available, apart from empirical data of existing airports.

Further work by Wirasinghe and Bandara [1990] led to an approximate closed form solution for the gate requirement which balanced the cost of delaying aircraft (due to lack of gates) against the cost of constructing and maintaining gate positions. For a given number of gates, $G$, with mean gate occupancy time and mean aircraft separation time of $\bar{T}$ and $\bar{S}$, respectively, the mean service rate (in aircraft/hr) of the gates is expressed as

$$
\mu=G /(\bar{T}+\bar{S})
$$


The total cost of delays and construction/maintenance can then be given as

$$
Z=\mu(\bar{T}+\bar{S}) C+W K
$$

where $C$ is the marginal capital, maintenance and operating cost of a single gate position per day, $K$ is the average cost of delay to airlines and passengers per aircraft per $\mathrm{hr}$, and $W$ is the total deterministic delay (in hours) to aircraft each day, due to lack of gates. They assume that $W$ is a function of $\mu$ and a number of other parameters. The optimal service rate can be obtained by minimizing $Z$ with respect to $\mu$ in equation 2.10 , and the optimal number of gates can be obtained by substituting the value of $\mu$ into Equation 2.6.

\subsection{3 - Other Measures}

Although the majority of decision support research done in assessing airport passenger convenience measures has concentrated on estimating walking distances, other measures have received attention in terms of quantitative modeling. One such measure is the size of aircraft departure lounges. Typical models for determining the required seating capacity of departure lounges use standard results from queuing theory based on assumptions of passenger arrival distributions and boarding rates. Paullin and Horonjeff [1969] used queuing techniques related to flows of passengers to calculate a maximum passenger accumulation statistic based on the time before departure that boarding commences. If the seating capacity of the lounge is set equal to the maximum passenger accumulation associated with the largest capacity aircraft servicing the gate, then the lounge will be able to accommodate all smaller flights as well.

The choice of the cumulative arrival curve for passengers departing on a particular flight is crucial to the calculation of the value for the maximum accumulation statistic. Wirasinghe and Shehata [1988] extend the deterministic queuing theory approach to include the case of a mix of different aircraft

and flight types (varying the shape of the cumulative arrival curves). Based on penalties for voluntary and 
involuntary standing versus sitting, the authors calculate a cost function in terms of the lounge capacity. The minimum of this cost expression elicits the optimal lounge area and seating capacity. Results are also presented for combining the lounge areas for several gates, which they claim can reduce the necessary area by up to 50 percent.

Quantitative research has also been applied to the area of customs inspections [Wirasinghe and Perera 1992]. Long delays often characterize customs stations due to insufficient staff or inadequate operational procedures for handling large numbers of arriving passengers in a short period of time. Although some waiting is inevitable, the authors report that excessive delays can be reduced by improving customs procedures (e.g. on-board clearance of passengers and scheduling "low risk" flights away from international processing facilities), and by establishing an optimal processing rate for passengers through customs checkpoints, $b^{*}$. The two costs associated with $b$, the cost to passengers of waiting and the cost of higher inspection rates (by adding additional inspection officers), decrease and increase as with higher values of $b$, respectively. The authors derived an expression for the total cost to the system, and found that the optimal inspection rate, $b^{*}$, from which $n p$ passengers are inspected is

$$
b^{*}=\left(\gamma_{w} b_{1} / 2 \gamma_{p}\right)^{1 / 2 n p}
$$

where

$$
\begin{aligned}
& b^{*}=\text { optimal inspection rate } \\
& b_{1}=\text { average rate at which one customs officer can inspect passengers, } \\
& n=\text { total number of passengers on the flight, } \\
& p=\text { fraction of passengers whose bags are inspected by the customs service } \\
& \gamma_{w}=\text { value of unit of waiting time per passenger, and } \\
& \gamma_{p}=\text { cost of inspecting passengers by a customs officer per unit time. }
\end{aligned}
$$

The preceding discussion is meant to provide an overview of the work that has been done in the area of analytic decision support for airport passenger building design. The next section describes work that has been done in developing computer-based simulation programs to aid in the detailed layout of a given configuration of passenger buildings. 


\section{4 - Simulation-Based Decision Support}

Mumayiz [1990] provides an overview of several major airport terminal simulation models, thought to be improvements over the graphical models, empirical data, and rules-of-thumb techniques still in use today. He identifies the three most common functional types of simulation models: analytic queuing models, accounting models, and time-dependent models. For the purposes of simulating the landside airport environment, discrete-event, time-dependent simulations are most commonly employed. Such simulations can be categorized as event-, activity-; or process-oriented, depending on how the environment is structured, and what computer language is used to implement the simulation.

The author defines four functional levels of simulation programs, according to the purpose, characteristics, degree of sophistication, detail and precision anticipated. Level I simulations are the simplest and most basic, where time-variation and stochastic elements of the system are not considered. Instead, fixed peak demand patterns at each part of the airport are used as inputs. Level IV simulations, by contrast, employ demand and service rates which are both probabilistic and explicit functions of time -necessary calculations thus involve a high degree of mathematical complexity. Levels II and III fall in between the two extremes, with more elements being represented probabilistically in Level III.

Airport simulation programs are primarily developed by universities for academic research, by industry for proprietary purposes, and by the government. At MIT, Fay [1971] used simulation techniques to evaluate alternative terminal designs, and later Pararas [1977] developed a more detailed simulation model of the terminal building used for design support of specific terminal facilities. Horonjeff [1969] "simulated" the airport terminal environment using deterministic queuing models to analyze passenger and baggage flows, which led to other, more specific models for aircraft stand utilization, passenger processing and aircraft movement.

Simulation models were developed to evaluate landside terminal capacity by Dunlay and McCullough [1975] at the University of Texas at Austin, which led to the development of the ACAP airport simulation model. In Florida, the AIRSIM event-oriented simulation model was developed to deal with individual passenger and baggage flows according to a fixed flight schedule. Work outside the U.S. 
has been done at Loughborough University in analyzing the behavior of passenger processing in airports, Monash University in Australia in simulating international terminal operations, and in Denmark for the quantitative evaluation of basic design strategies for expansion plans of the Copenhagen/Kastrup airport [Mumayiz 1990]. Transport Canada has also sponsored research at several universities across Canada to study the essential functions of passenger processing facilities in the landside terminal area.

Although there exist several industry-developed simulation models for use in airport terminal planning, the proprietary nature of these programs prevents the widespread publication of their contents. Nevertheless, government agencies such as the FAA in the United States, the Canadian Air Transportation Administration (CATA), and the British Airports Authority (BAA) sponsor the development of their own simulation models and/or acquire industry-developed simulations for use in the public sector. The FAA was responsible for sponsoring much of the development work for ACAP, the Airport Landside Model (H.H. Aerospace), and the improvement of ALSIM, acquired from the Bechtel Corporation. CATA has developed the Calgary model which employs time-oriented queuing models, and Transport Canada has developed general simulation models for gate assignment, passenger and baggage flows within a terminal, and for studying ground transportation. The BAA has also developed simulation programs in-house for studying various passenger processing facilities.

\section{5 - Lesson from Literature Review}

In Section 2.1 we identified a gap in the available methods of decision support for Step One in the development of the Terminal Area Plan. The fact that a gap exists does not in itself motivate the need for work in that area. Based on the review of the available literature, however, it should be evident that there does exist a need for a fast and flexible decision support methodology to aid airport planners in selecting an initial configuration of passenger buildings. This need motivates the development of some sort of computer-based tool which can take advantage of available technology with its capacity to perform millions of simple calculations per second. Such a tool could provide specific information quickly, 
making it as easy to use as reference manuals, yet it would be more powerful in its ability to make objective comparisons based on actual quantitative data.

In addition to being powerful in terms of speed, the tool must also be flexible enough to assess the potential performance of several very different configuration concepts. Such flexibility is already available in the detailed Monte Carlo simulation programs described in Section 2.4; however, as mentioned previously, long setup times and detailed input requirements make these programs limited by the total time (from setup to results) required to evaluate each configuration type. Even with faster, more powerful microprocessors, the time required for the data acquisition remains an unalterable and limiting factor for increasing speed. The need for a new decision support methodology is represented graphically in Figure 2.10.

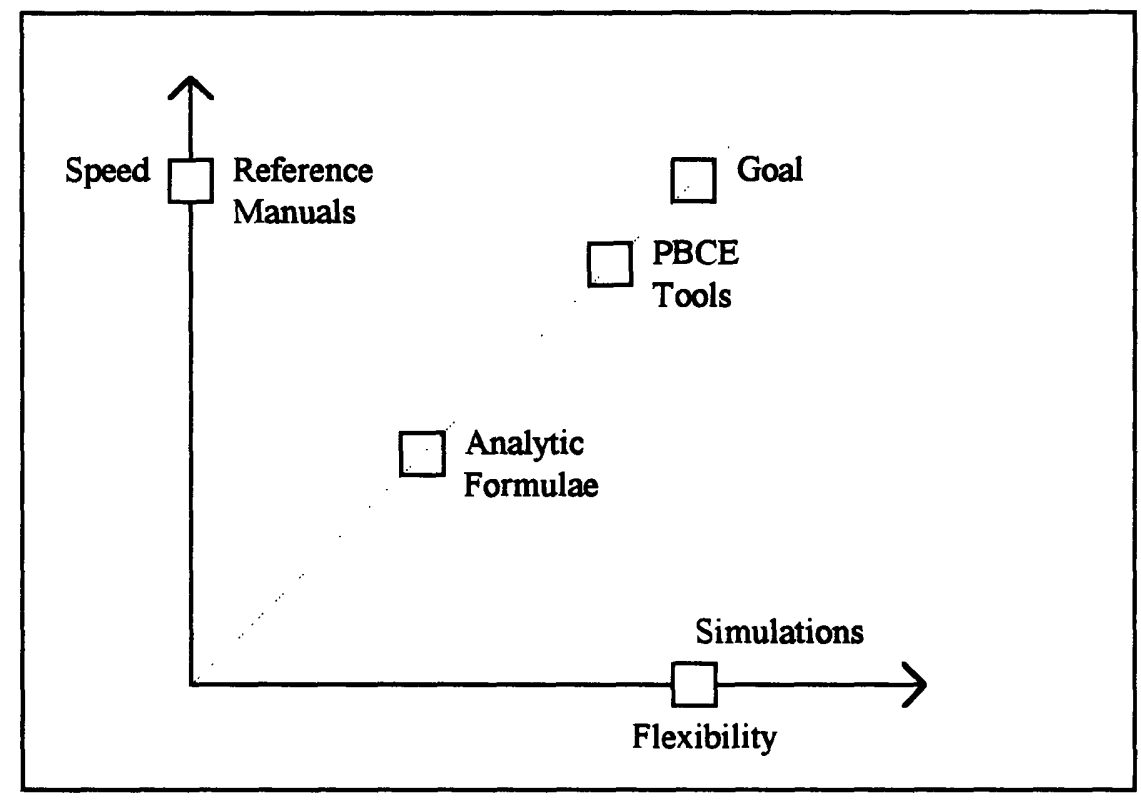

Figure 2.10 : Graphic Representation of Decision Support

The horizontal axis represents the degree of flexibility associated with a particular decision support methodology, in terms of the number of different conditions that can be considered. The vertical axis represents the speed with which the methodology can produce results. The ultimate (perhaps 
unattainable) goal is some sort of tool that can combine the ease and speed of reference manuals and texts with the flexibility of the detailed Monte Carlo simulation programs.

As mentioned previously, although reference manuals provide quick decision support, they are limited in the conditions they can consider (High Speed, Low Flexibility). Simulation programs, on the other hand, provide a great deal of flexibility (in terms of the conditions they can consider), but are slow due to large setup times (Low Speed, High Flexibility). Analytic formulae techniques such as those described in Section 2.3 provide greater flexibility than reference manuals because they can consider several standard concepts over a range of different conditions. In addition, these techniques are generally faster than simulation programs due to the simplifying assumptions made to represent the different configuration types; however, these assumptions (necessary to obtain solutions) limit their flexibility versus the simulation programs. Moreover, the time to characterize different concepts geometrically (in order to establish the defining expressions) limit their speed versus reference manuals and texts.

The goal of the PBCE tools developed and applied in this thesis is to move the state of the art in terms of speed and flexibility closer to the ultimate goal (High Speed, High Flexibility). Such a tool is only now possible due to the availability of inexpensive computer technology. Thus, we are now able to adequately address an issue in airport planning that was previously unaddressable. The remaining chapters of this thesis demonstrate how a spreadsheet-based, "object-oriented" PBCE tool can be implemented and used to aid planners in the selection of airport passenger buildings. 


\title{
Chapter 3
}

\section{Airport Configuration Concepts}

\author{
Introduction
}

The number of different airport terminal configurations is seemingly as large as the number of airports, yet virtually all can be placed into a few primary categories [de Neufville 1976] based on underlying philosophies of function (e.g. gate arrival, finger-pier, satellite, and midfield). Furthermore, each of these primary configuration types can be divided into centralized and decentralized sub-categories, depending on the operational philosophy of check-in, baggage handling, and other service facilities. In general, centralized configurations are characterized by a single, common area containing check-in, baggage handling, and other auxiliary facilities for all airlines, whereas decentralized configurations, sometimes known as "unit terminal" concepts, have separate, individual facilities for airlines located in separate buildings.

The primary configuration types are the result of an evolutionary process which began with the formation of scheduled airlines in the 1930's [Blankenship 1974]. The first terminal buildings were designed to provide a direct interface between airport access/egress modes and the aircraft. Accordingly, the gate arrival, or linear design allows for very short distances between curbside and departure areas. 
Increases in the volume of passenger traffic and the resulting need for more aircraft stands brought about the second generation of terminal configurations, which included the introduction of piers. Piers could be attached to existing linear terminals, and could increase the number of gates available at an airport. Originating and terminating passenger walking distances necessarily increased, however, as aircraft stands had to be reached through a series of corridors.

When further increases in passenger traffic volumes could no longer be handled by adding piers to existing terminals, a third generation of airport configuration concepts evolved. The major difference of the new generation of passenger building configurations was that departure concourses were now completely disjoint from the original "terminal" building, and access to them was achieved via above- or below-grade connectors, transporter buses, or midfield people mover systems. The introduction of mechanical devices such as moving sidewalks and shuttle buses were necessary, given the increased distances between a passenger's access/egress point and the aircraft interface. Strict adherence to a single configuration type is not common. Indeed, many "hybrid" configurations exist which borrow operational aspects from two or more of the standard configuration concepts. A discussion of the primary types of configuration concepts, including examples, follows.

\section{1 - The Gate Arrival Configuration}

The oldest and simplest configuration concept is the gate arrival, or linear design. As mentioned previously, the gate arrival configuration is attractive due to the direct interface between airport access/egress and aircraft. The most traditional design for small, centralized linear terminal configurations is a single building containing all necessary passenger services, including check-in, baggage, security stations, etc. Figure 3.1 illustrates one example of a centralized gate arrival configuration, the new International Terminal 5 at Chicago / O'Hare Airport, which opened in May of 1993. 


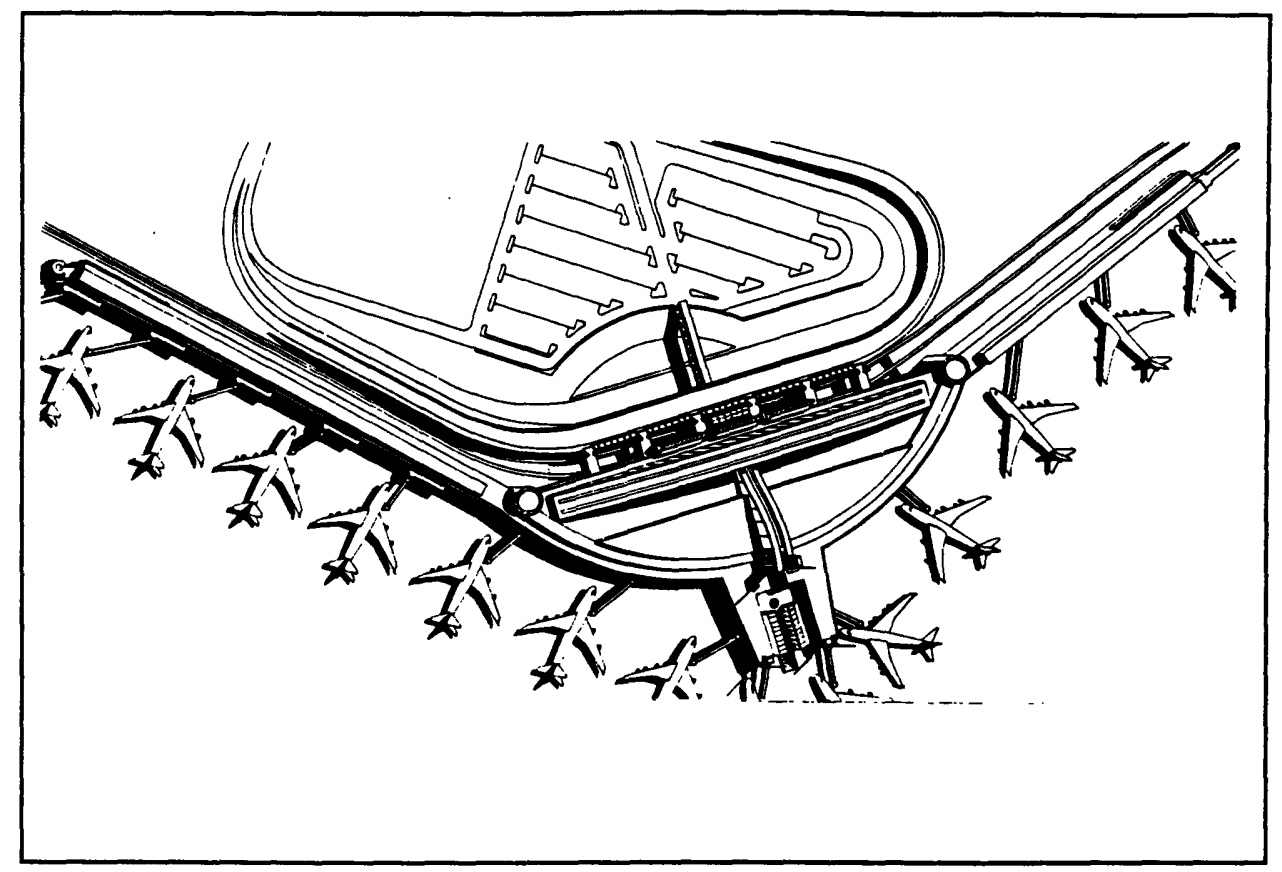

Figure 3.1: Terminal 5 at Chicago / O'Hare International Airport ${ }^{1}$

The centralized gate arrival design is particularly suited for shuttle service terminals, for which the bulk of passenger traffic tends to be business travelers who generally do not carry much baggage and tend to arrive at an airport close to departure time. Such passengers do not require much in the way of baggage handling equipment and waiting area lounges, and so the simple, single building concept is appropriate. Shuttle buildings employing a centralized gate arrival design are present at Washington National (1970) and New York / LaGuardia (1958).

In order to take advantage of the convenience of direct access to aircraft, larger gate arrival configurations can be arranged in a linear procession of unit terminals, each with its own set of passenger processing facilities. Such decentralization allows each terminal building to act as an isolated system, and thus the individual terminal blocks can be smaller than if a single, centralized block were used. Examples of decentralized gate arrival configurations include Dallas/Ft. Worth International (1973), the terminals for Sapporo at the Shin Chitose Airport in Japan (1992), and Kansas City International (1972), shown in Figure 3.2. Although each "unit terminal" is, in fact, a semi-circle, for passengers the configuration is no

\footnotetext{
1 Source: [Momberger 1993a], p. 11.
} 
different topologically than a series of simple rectangles. For aircraft, however, the circular design more easily facilitates the placement of aircraft, where wingspans are the controlling parameter for airside building frontage. The design also has increased curbside frontage on the landside and allows for parking in the middle of the semi-circle.

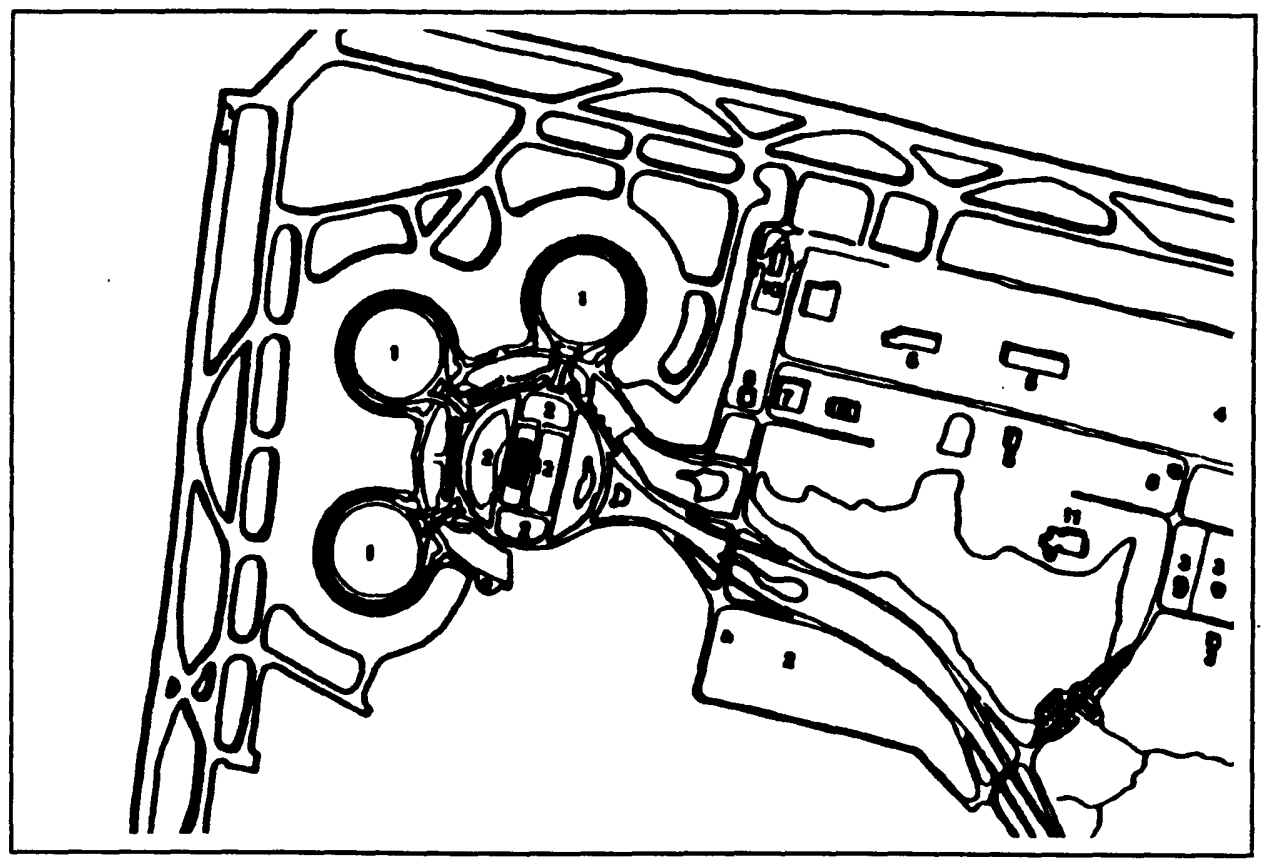

Figure 3.2: Kansas City International Layout ${ }^{2}$

\section{2 - The Pier Configuration}

The second generation of terminal configurations, represented by the pier concept, introduced a new method of passenger processing by the airlines. Rather than having one common passenger-holding facility, airlines moved toward having separate holding facilities for each flight. Passengers could then be processed and held in lounges directly adjacent to aircraft. Although originally intended to provide extra

2 Source : [Massport 1988]. 
frontage for aircraft stands, other operational advantages intrinsic in the pier concept helped it become the most popular design in the United States [Blankenship 1974].

When utilized in a two-level design, the pier concept allows for the separation of several arrival and departure functions, including independent arrival and departure curbs, ticketing, and baggage services. Such a scheme can also be used to keep enplaning and deplaning passengers separate. Such a separation scheme has since been applied to linear, satellite, and transporter configurations as well. Pier configurations can be arranged in both centralized and decentralized concepts. Figure 3.3 illustrates the centralized, parallel pier configuration of the main terminal at New York / LaGuardia Airport (1939). Other examples of centralized pier configurations include Frankfurt/Main, Terminal Mitte (1972), and Paris, Orly-Sud (1961), and Orly-Ouest (1971).

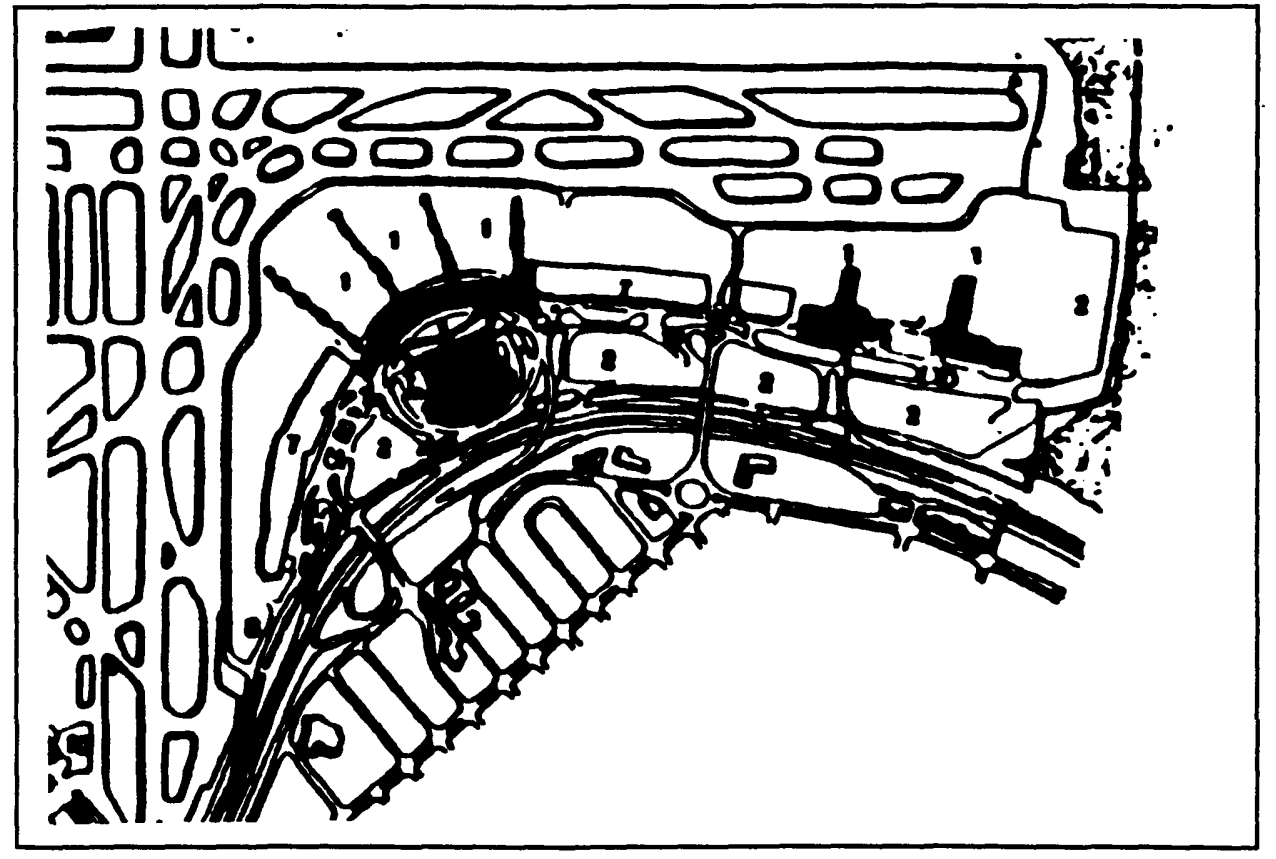

Figure 3.3 : New York/LaGuardia Main Terminal Layout ${ }^{3}$

${ }^{3}$ Source : Ibid. 
Originating passengers enter through a common terminal block and reach departure concourses through a series of corridors. Pier configurations can also be decentralized into a unit terminal concept, such as the Philadelphia Airport (1977), shown below in Figure 3.4. Each pier has its own terminal block which houses the necessary passenger processing facilities, which reduces walking distances for originating and terminating passengers.

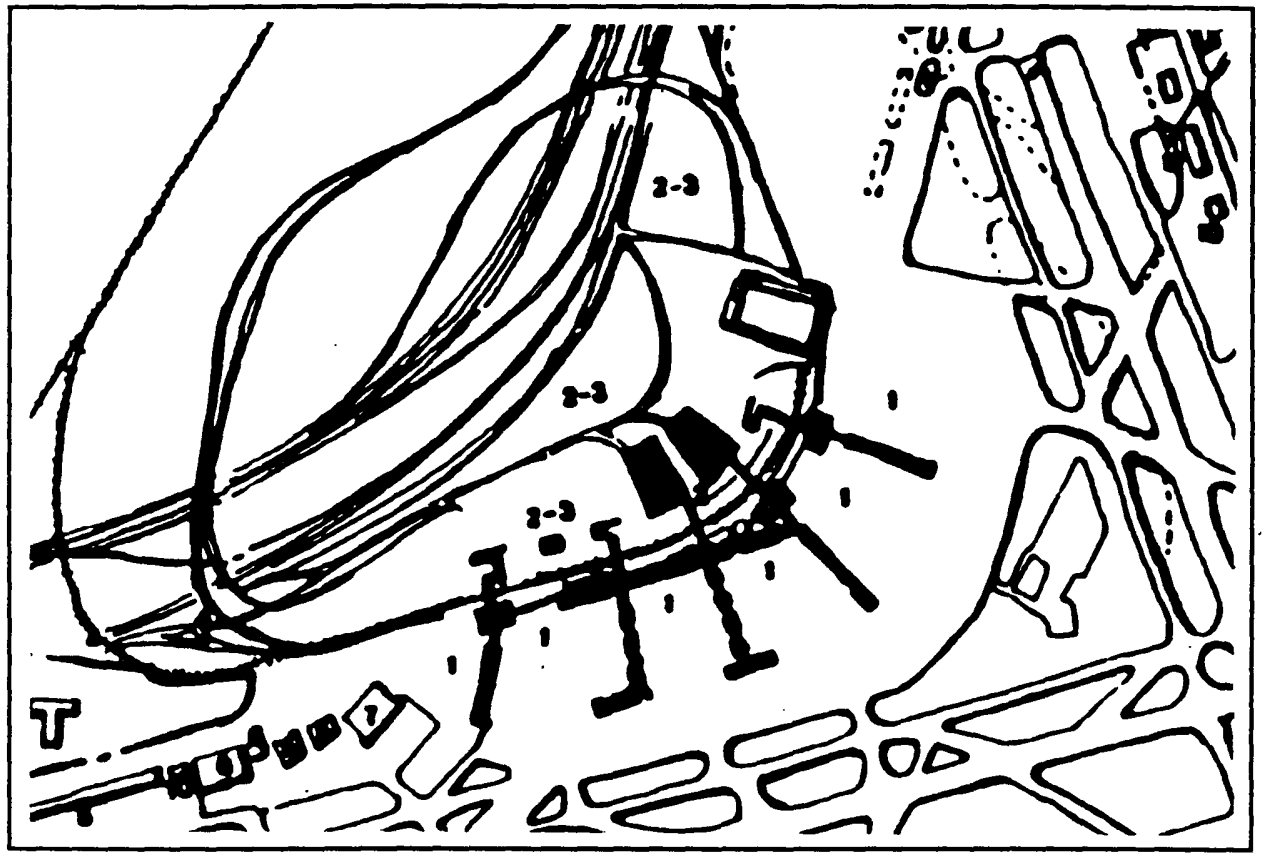

Figure 3.4: Philadelphia International Layout ${ }^{4}$

Transfer passengers whose departure gate is not located with their arrival concourse may have increased walking distances due to the decentralization, however. Decentralized pier configurations can also be found at Chicago / O'Hare International (1963), and London / Heathrow Terminals 1 (1968), 2 (1955), and $3(1970)$.

\footnotetext{
${ }^{4}$ Source: Ibid.
} 


\section{3 - The Satellite Configuration}

The third generation of passenger building configurations is the satellite concept. The concept was originally designed to improve aircraft maneuverability by separating aircraft stands from the main terminal buildings, thus (potentially) reducing apron area congestion. To further facilitate maneuverability, many satellite concepts are constructed with below-grade connectors (from the terminal buildings to the satellites), allowing aircraft to be parked around the entire satellite perimeter without creating cul-de-sacs during entry and exit. Landside terminal buildings house most of the passenger processing facilities, though some may be located within the satellite. Satellite concourses can take many different shapes, including circles, rectangles, "T" and " $\mathrm{Y}$ "-shapes, as well as various "hybrid" combinations.

Again, satellite configurations can be configured in both centralized and decentralized concepts. Figure 3.5 shows an example of both concepts combined, at the New York / Newark International Airport (1973). Considered as a whole, the Newark configuration is decentralized, containing three individual terminal blocks arranged around an oval road system. Taken separately, however, each terminal block can be thought of as its own centralized satellite configuration, with three circular satellites arranged radially about a common terminal building. Other examples of configurations which include satellites are the Central Terminal at San Francisco International (1983), the Cologne/Bonn Airport (1970), and Los Angeles International (1961)

\section{4 - The Transporter Configuration}

Transporter configurations are similar in operational philosophy to some older, simpler designs in which passengers were taken by bus to aircraft parked in positions without fixed facilities. In the transporter concept, apron passenger vehicles take passengers directly from the terminal building to the aircraft, without exposing passengers to the outside. Apron passenger vehicles vary from airport to airport, but essentially can be placed into fixed and variable height (non-elevating and elevating) 
categories. Non-elevating transporters are similar to street vehicles, modified with a stair boarding device "to complement the operation for passenger boarding, crew access, cabin service access, and emergency egress." [Parsons 1975, p. 3-10] Varying sizes of aircraft and architecture, however, make variable height transporters more desirable, particularly in dealing with seasonal planning (when aircraft mixes can vary significantly due to charter operations). Figure 3.6 shows the transporter configuration of the Washington / Dulles International Airport (1962).

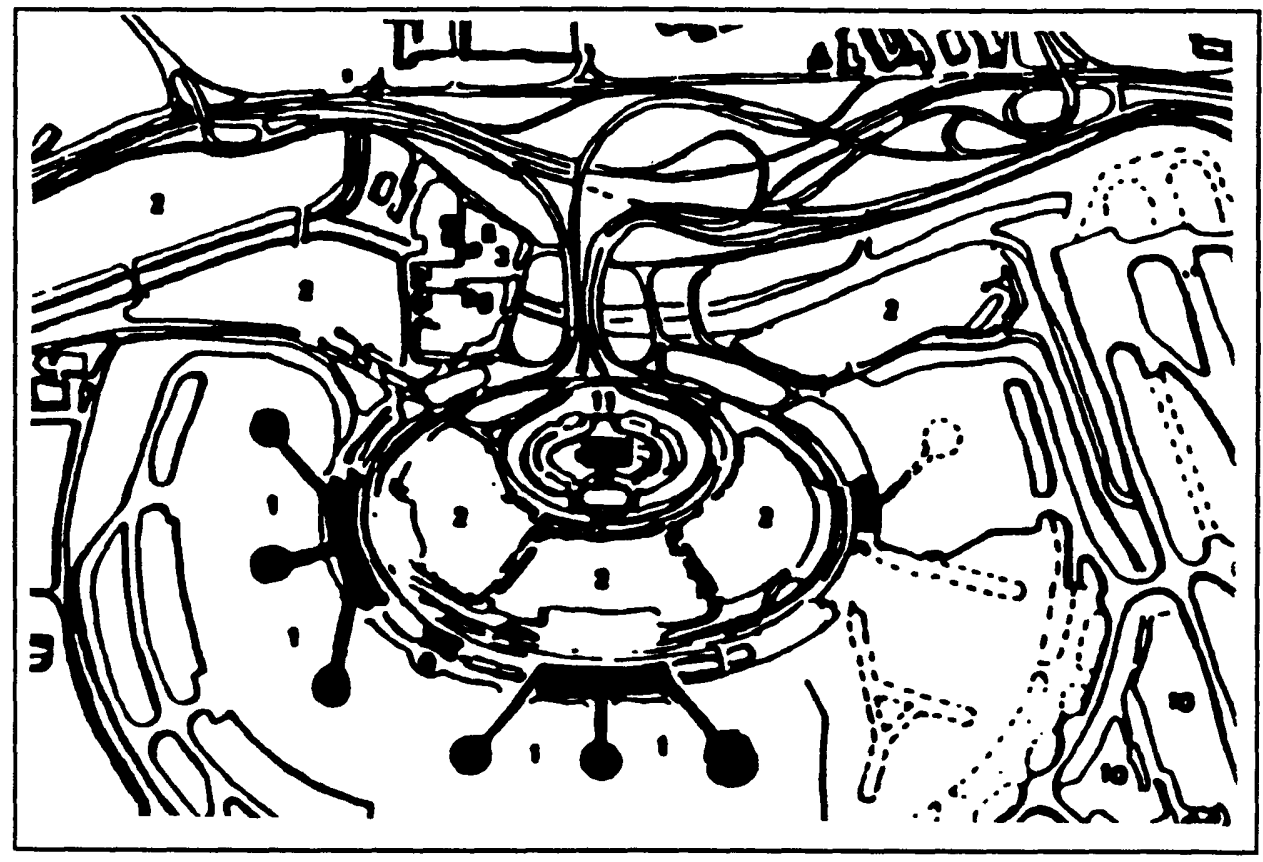

Figure 3.5: New York / Newark International Layout 5

One landside advantage of the transporter concept is the reduced requirement for fixed facilities beyond the terminal block. Although transporter airports typically do have some facilities on the airside similar to satellite concourses (for apron managers, airline personnel, etc.), they are generally less complete in the facilities they accommodate. Increases in passenger loads are often handled by employing additional transporters rather than by expanding existing piers or satellites. On the airside, there is

${ }^{5}$ Source : Ibid. 
significant advantage to parking aircraft away from the terminal building due to the elimination of timeconsuming taxi-in/taxi-out operations by tow trucks. Among the disadvantages cited on the passenger side is that the departure of the transporter is equivalent to the aircraft departure from the concourse, which reduces the "slack" time afforded last-minute passengers trying to reach their departure gate. Airlines cite the labor costs for drivers and the maintenance costs for the upkeep of the vehicles.

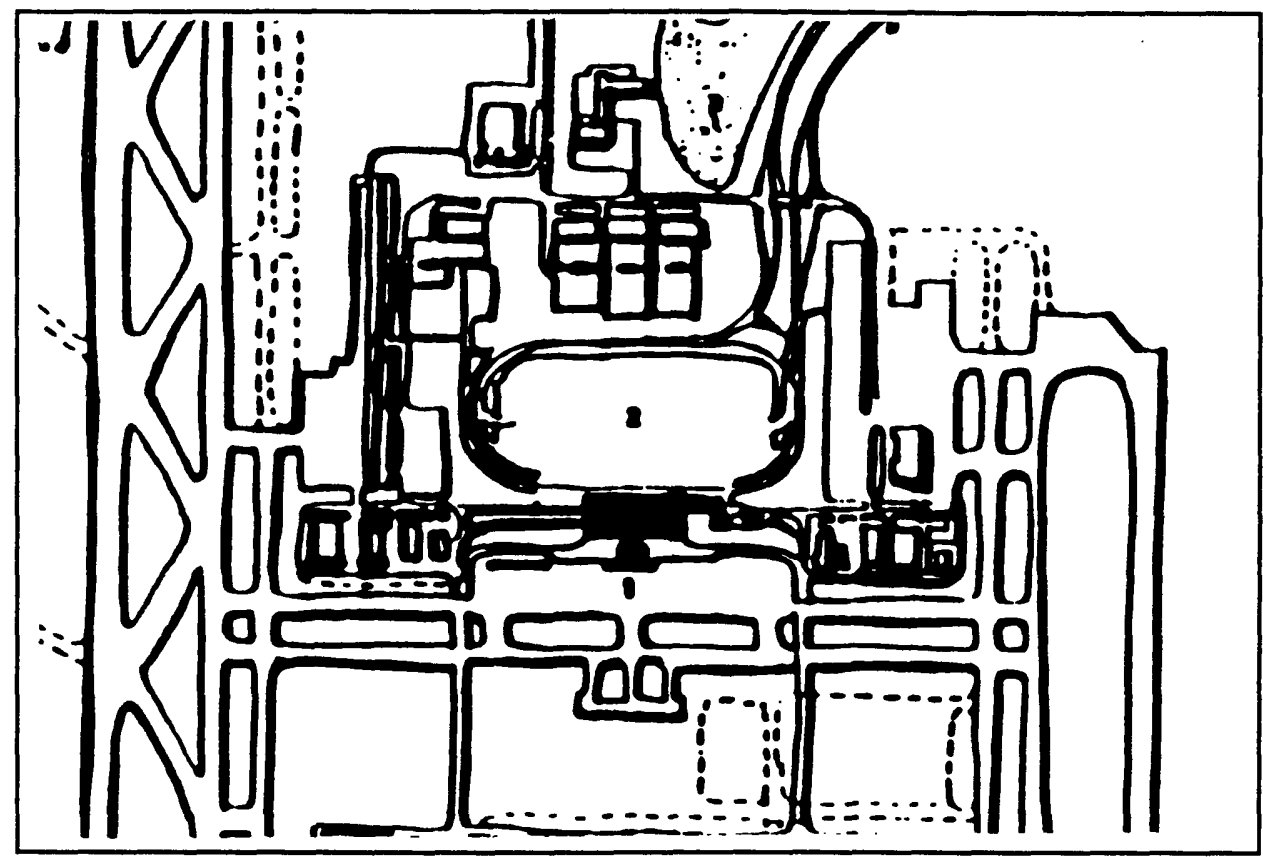

Figure 3.6 : Washington / Dulles International Layout ${ }^{6}$

\section{5 - The Midfield Configuration}

The most recent evolution of configuration concepts is known as the midfield design. Like the third generation satellite concept, individual passenger building concourses are disjoint from the terminal building, and access to departure gates typically involves some form of people moving device to avoid

\footnotetext{
${ }^{6}$ Source : Ibid.
} 
excessively long walking distances. Concourses can be arranged in a variety of patterns, depending on geographic limitations and operational philosophy.

Figure 3.7 shows the layout of Atlanta's Hartsfield Airport (1980), which illustrates one of the more common midfield configurations known as the parallel linear design -- a similar layout is being used at the new Denver International Airport (exp. 1994). Originating passengers enter a centralized landside terminal building and go through passenger processing to an underground tram that takes them to the center of their departure concourse. Transfer passengers can also use the tram to get between midfield concourses.

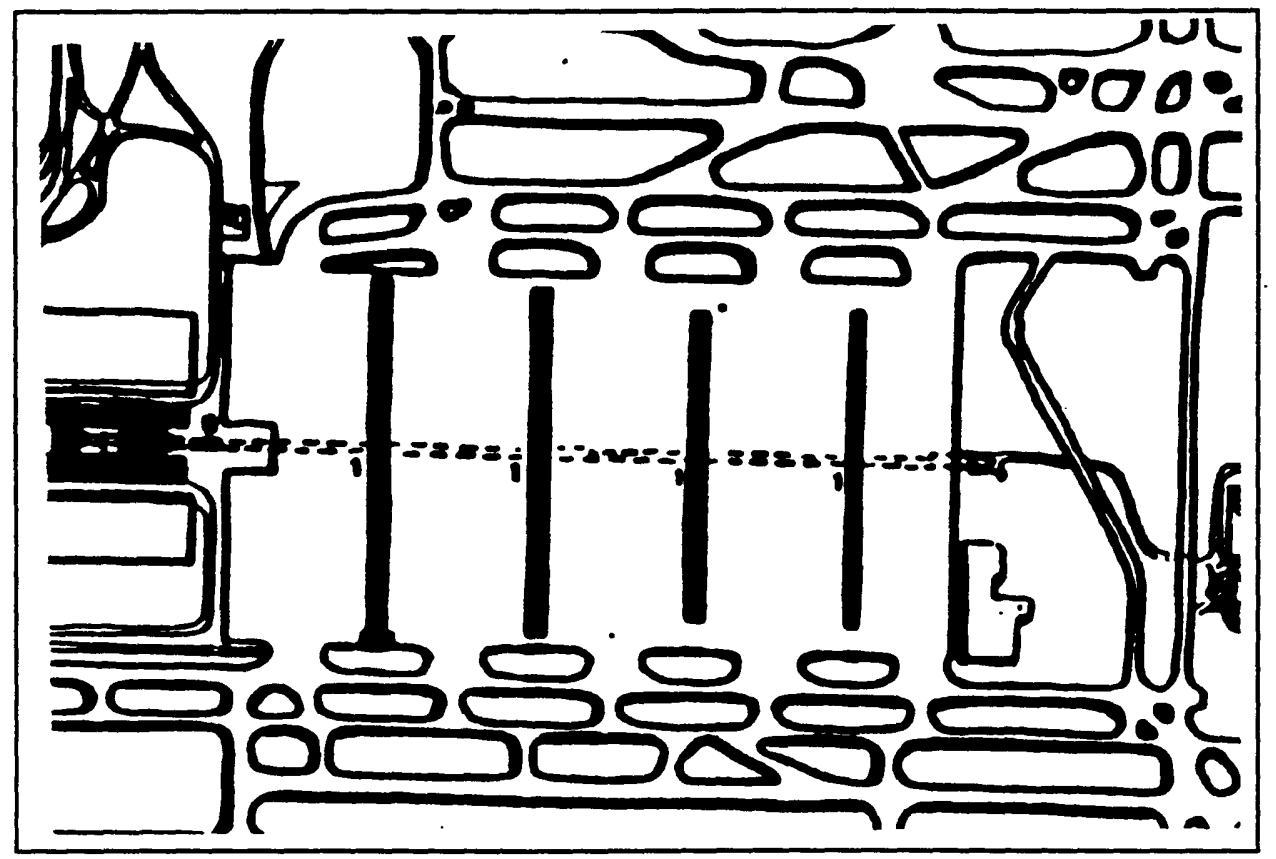

Figure 3.7 : Atlanta / Hartsfield Layout ${ }^{7}$

Because the tram is below-grade, aircraft can be parked on all sides of the rectangular concourses and taxiways do not end in cul-de-sacs. Another example of a centralized midfield configuration is the New Pittsburgh Airport (1992), with a single midfield concourse shaped like an "X".

\footnotetext{
${ }^{7}$ Source : Ibid.
} 
Midfield configurations can also be arranged in a decentralized fashion, as shown in the layout for the Seattle-Tacoma Airport (1980) in Washington state (Figure 3.8). In Seattle, an underground transit system is split into two "loops", a North Loop and a South Loop, each of which travels to a rectangular midfield concourse and back to a bent pier attached to each of the two unit terminals. Passengers connecting within the same concourse can again remain midfield, whereas those connecting between concourses can transfer to the other loop through the respective terminal building. Because of the advantages of midfield configurations for connecting passengers, they are becoming an increasingly popular concept for airports expecting a high level of transfer traffic [de Neufville 1994].

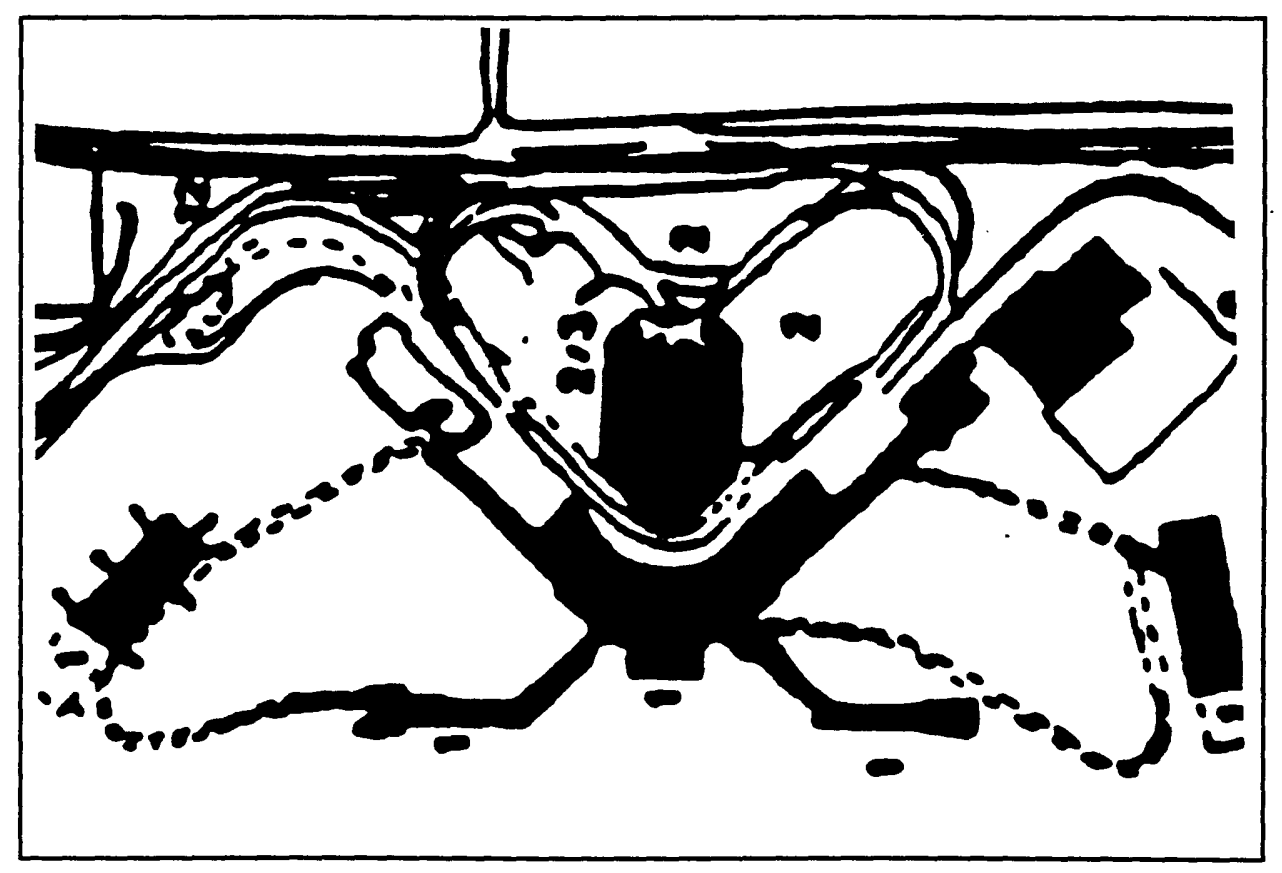

Figure 3.8: Seattle-Tacoma Layout ${ }^{8}$

\footnotetext{
8 Source : Ibid.
} 


\section{6 - Hybrid Configurations}

Standard configurations in their purest form are rare. Indeed, many airport configurations are in fact a mixture of different operating philosophies. Seattle-Tacoma operates as a decentralized midfield configuration, but there are also two finger piers extending off each of the primary terminal buildings. Although the main terminal at New York / LaGuardia Airport has a centralized parallel pier concept, there are other buildings including a gate arrival shuttle terminal and another finger pier terminal.

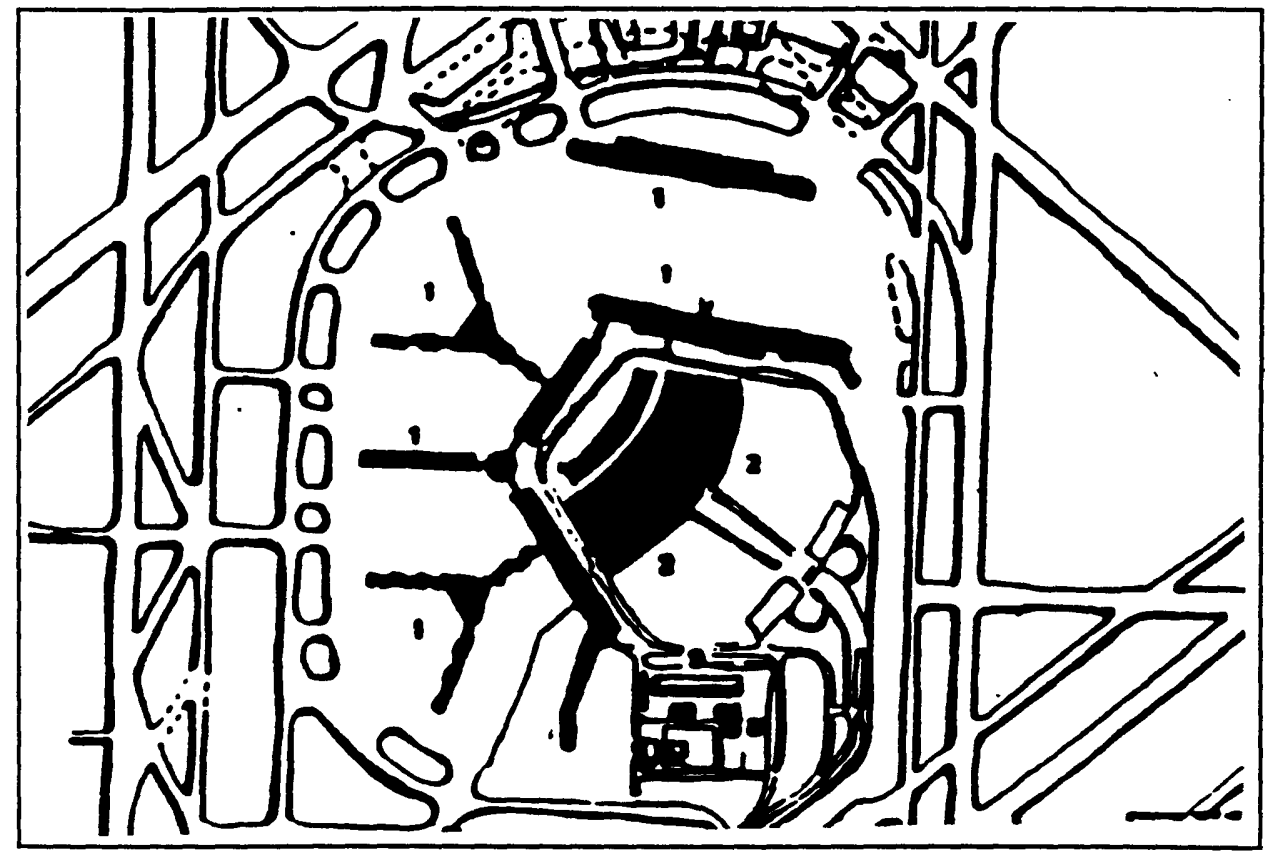

Figure 3.9 : Chicago / O'Hare International Layout ${ }^{9}$

So-called "hybrid" configurations take on the operational philosophies of two or more of the standard configuration concepts. Frequently, hybrids are created not by design but by expansion of existing facilities. For example, consider the layout of the Chicago / O'Hare International Airport shown in Figure 3.9. The airport is characterized by a series of pier terminals, arranged in a semi-circular

${ }^{9}$ Source: Ibid. 
fashion. The four major piers are either rectangular or "Y"-shaped, and extend from unit terminals located at their base. The newest passenger building at Chicago / O'Hare (United Airlines' Terminal 1), however, departs from the traditional pier scheme. A rectangular satellite is connected to its unit terminal by an underground connector.

Another example of a hybrid-by-expansion configuration is Boston's Logan International (Figure 3.10). Similar to Chicago / O'Hare, Logan has unit-pier terminals arranged in a semi-circle, including the North Building (1967) which houses two "T"-shaped piers and the South Building (1976) which houses two straight finger-piers. Logan departs from this pure form, however, with its shuttle terminal, Terminal A (1969), which is characterized by the gate arrival concept. On the East Coast, one of the largest citypair markets for air travel is Boston - New York City, which is heavily populated by business passengers. As mentioned previously, the direct access philosophy of the gate arrival design is well suited for such traffic, thus justifying its departure from the other finger-pier concepts.

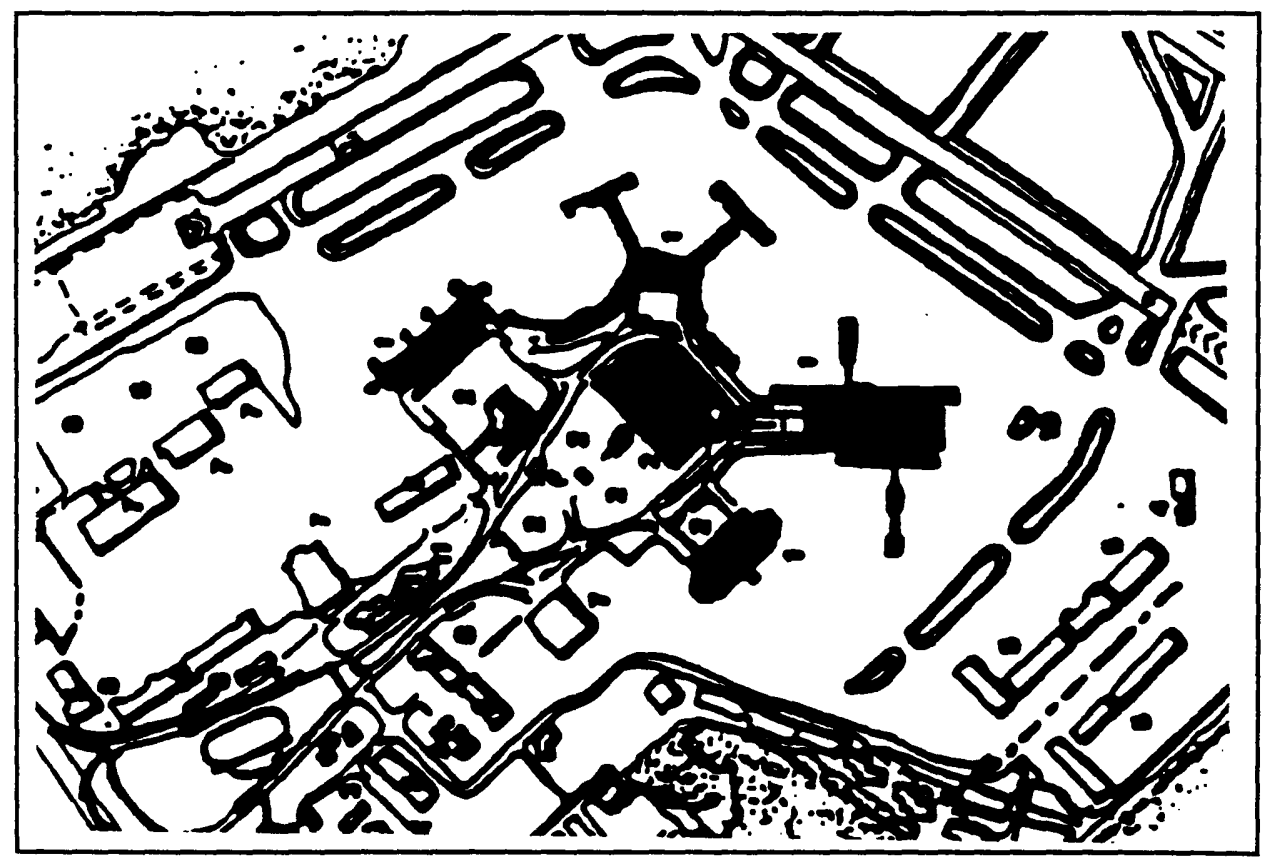

Figure 3.10: Boston Logan International Layout ${ }^{10}$

10 Source: Ibid. 
Some hybrid concepts are created by design rather than merely by expansion. Consider the proposed design of the new Hong Kong / Chek Lap Kok Airport (Figure 3.11). Like the New Pittsburgh Airport, Chek Lap Kok will operate as a midfield airport with an "X"-shaped concourse. Additionally, however, the main building will also have a "Y"-shaped finger-pier directly on the other side of the passenger processing facilities. It can thus also operate as a gate arrival or linear concept. Such a design makes intuitive sense if one considers the separation of the two primary passenger types predicted to use the Chek Lap Kok Airport.

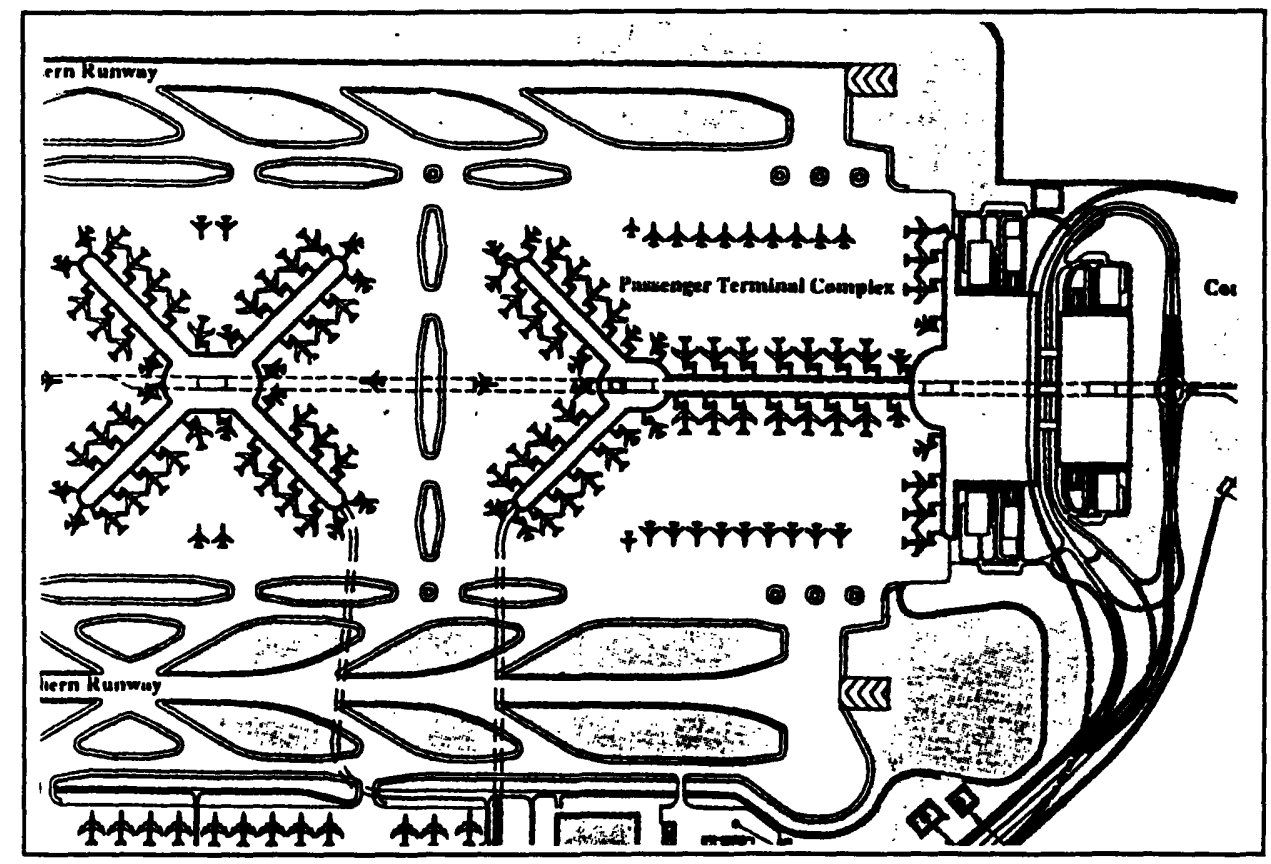

Figure 3.11 : Proposed Hong Kong / Chek Lap Kok Layout ${ }^{11}$

For short-haul (local) originating and terminating passengers, the gate arrival concept minimizes walking distances by providing a direct interface between ground access/egress and aircraft. Due to its geographical location, however, Hong Kong is also a convenient connection point for traffic within the Pacific Rim. For long-haul connecting passengers who do not require intermediate services, the "X"-

11 Source: [Momberger 1993b], p. 11. 
shaped concourse provides direct access between their arrival and their departure gates, without the need to leave the midfield environment. Long-haul, international originating (terminating) passengers will, in general, experience longer distances between curbside and their aircraft; however, these passengers tend to arrive at the airport earlier than domestic (short-haul) passengers, and thus they will have more time to traverse the path to their departure gate.

The Hong Kong example illustrates an important advantage to hybrid-by-design configurations; namely, the combination of the most desirable features from two or more standard configuration types in an attempt to match operational concept with anticipated passenger traffic. By utilizing both a gate arrival and a midfield concept, the airport is potentially able to provide a better level of service to both passenger types, rather than sacrificing service to one for another. In the short-term, this synthesis of concepts is likely to outperform any single standard configuration, given the passenger traffic anticipated.

In the long-run, however, the hybrid-by-design concept is likely to provide even further advantages. Because of its flexible design, major changes in passenger traffic can be accommodated without severely degrading performance [de Neufville 1976]. For example, if Hong Kong continues to be an increasingly popular hub/transfer point in the Pacific, another " $\mathrm{X}$ "-shaped midfield concourse can be constructed to handle higher transfer passenger loads. If, on the other hand, there is an increase in originating and terminating passenger traffic, the linear frontage can be extended to provide more aircraft stands.

Such flexibility in design is rare among current airports, and reflects the historical absence of a clear design strategy to plan for configuration robustness to major change. In the next chapter we present a detailed characterization of different standard terminal concepts to provide the underpinnings of our new approach to terminal configuration design. By establishing a formal means of comparing and contrasting terminal types, we can gain insight into the most desirable features of each when trying to create both pure and hybrid configurations. 


\title{
Chapter 4
}

\section{Representing Passenger Building Configurations Geometrically}

\author{
Introduction
}

In order to compare and contrast different passenger building configurations it is necessary to standardize the concept types so that differences in performance estimates come from the geometry inherent in each concept. This chapter develops possible geometric representations of the passenger building configurations described in Chapter 3. From these representations it is possible to obtain the distance between any two points, either by summing the distances associated with different components of a passenger's path or by overlaying a Cartesian coordinate grid on the layout and determining distances directly. This method is also useful when trying to assess the potential performance of non-standard configuration types. By using the distance data along with certain assumptions regarding individual passenger paths, we can obtain matrices to represent the absolute distances walked by each passenger category considered for analysis. Bandara [1989] has developed a "vocabulary" for describing many configuration concepts, and we use this vocabulary as a basis for our geometric representations wherever 
possible. For those concepts not previously considered, we add to Bandara's vocabulary using consistent notation.

\section{1 - Centralized Configurations}

Centralized terminals can be characterized by a single common area, or terminal block, which contains check-in, baggage handling, and other auxiliary services. Bandara [1989] defines the following global parameters used for all concepts :

$$
\begin{aligned}
& G \quad=\quad \text { total number of aircraft stands (gates) in the airport } \\
& n=\text { number of individual concourses } \\
& g_{n} \quad=\quad \text { number of gates within concourse } n \text { ( } g \text { if the number in each concourse is constant) } \\
& b_{p} \quad=\text { distance walked by originating and terminating passengers within the terminal block } \\
& S_{g} \quad=\text { distance between adjacent aircraft stands (gate spacing) }
\end{aligned}
$$

For his analyses, Bandara assumed $b_{p}$ and $S_{g}$ are constant for a given $G$. Thus, the size of the terminal block and the spacing between gates remain constant for all possible configuration geometries (combinations of $n$ and $g$ such that $n g=G$ ). Such assumptions are necessary to obtain the single expression (in terms of $n$ ) he uses to obtain the "optimal" configuration geometry for a given set of conditions. As mentioned previously, however, distance values could be obtained directly and do not depend on such assumptions.

\subsection{1 - The Gate Arrival Concept}

Figure 4.1 illustrates two possible geometric representations of the centralized gate arrival concept, one in which the total number of gates, $G$, is odd [Figure 4.1(a)], and one in which $G$ is even [Figure 4.1(b)]. If one uses Bandara's assumption that $b_{p}$ and $S_{g}$ are constant, originating passengers can be assumed to walk a distance $b_{p}$ through the terminal block, plus $S_{g}$ times the number of aircraft stands their departure gate is from the center of the terminal block (when $G$ is odd). If $G$ is even (and gates are 
arranged symmetrically about the center of the terminal block), the nearest departure gate would be located $\frac{s_{g}}{2}$ from the center [Figure 4.1(b)].

Since $S_{g}$ is constant, direct transfer passengers are assumed to walk some multiple of $S_{g}$ from their arrival gate to their departure gate. Indirect transfer passengers, on the other hand, must include in their paths some intermediate service point. Bandara [1989] assumes this service point is located within the terminal block, so that indirect transfers would walk from their arrival gate through the terminal block (for intermediate services), and then on to their departure gate. Under such an assumption, indirect transfer passengers departing from their own arrival gate would walk a distance equal to twice that of an originating passenger departing from that gate. Note that this method of determining distances can be applied only to "idealized" configurations, and is not easily generalized.

\subsection{2 - The Parallel Pier Concept}

Figure 4.2 illustrates a simple representation of the centralized parallel pier concept. Departure concourse centerlines are separated by some spacing $S$, the distance of which is governed by the number (and size) of taxiways and the spacing requirements for aircraft. Bandara [1989] cites the following spacing recommendation for nose-in parking,

$$
S=2 L_{a}+W_{p}+m W_{t}+(m+1) C_{t}
$$

where :

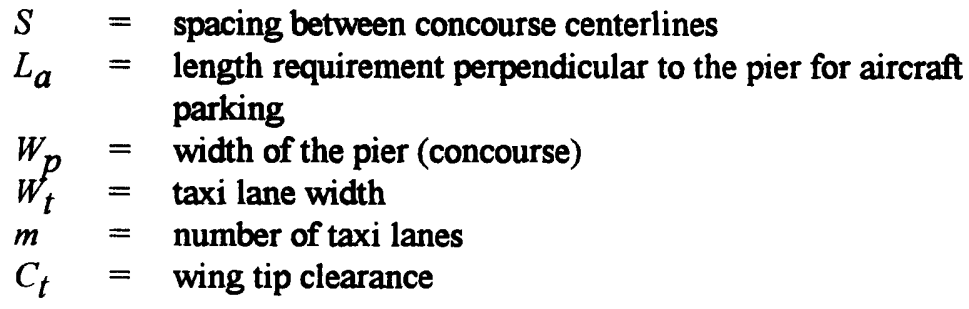

Such a definition, however, works only for a very specially defined condition (Figure 4.3). Nevertheless, for any given $S$, the distances walked by each passenger category can be obtained in a similar fashion to 
those in Section 4.1.1, either by assuming constant $b_{p}$ and $S_{g}$ values, or by enumerating individual passenger paths on a Cartesian grid.

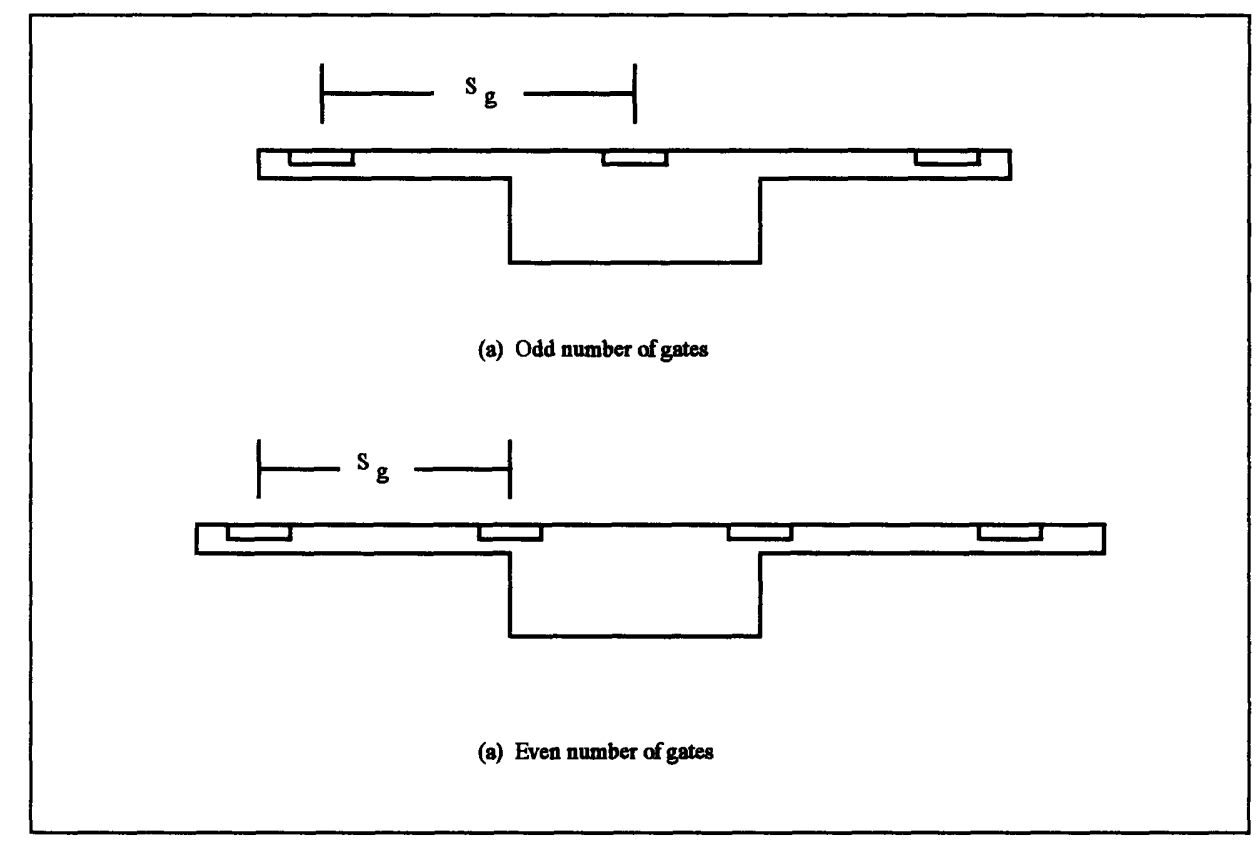

Figure 4.1: Centralized Gate Arrival Concept

\subsection{3 - The Satellite Concept}

Centralized satellite configurations are characterized by a single terminal block surrounded by concourses which can take various geometric shapes (Figure 4.4). One way to represent satellite configurations is to assume that satellites are arranged radially about the terminal block (placed as close together as possible), separated by some mandatory spacing, $S$, again governed by taxilane widths and aircraft wingspans. In order to maintain mandatory spacing both among satellites and between each satellite and the terminal block, the length of the connectors from the terminal block to the satellites can be varied. Bandara [1989] defines the parameter $B$ (subscripted to denote the type of satellite) such that, 


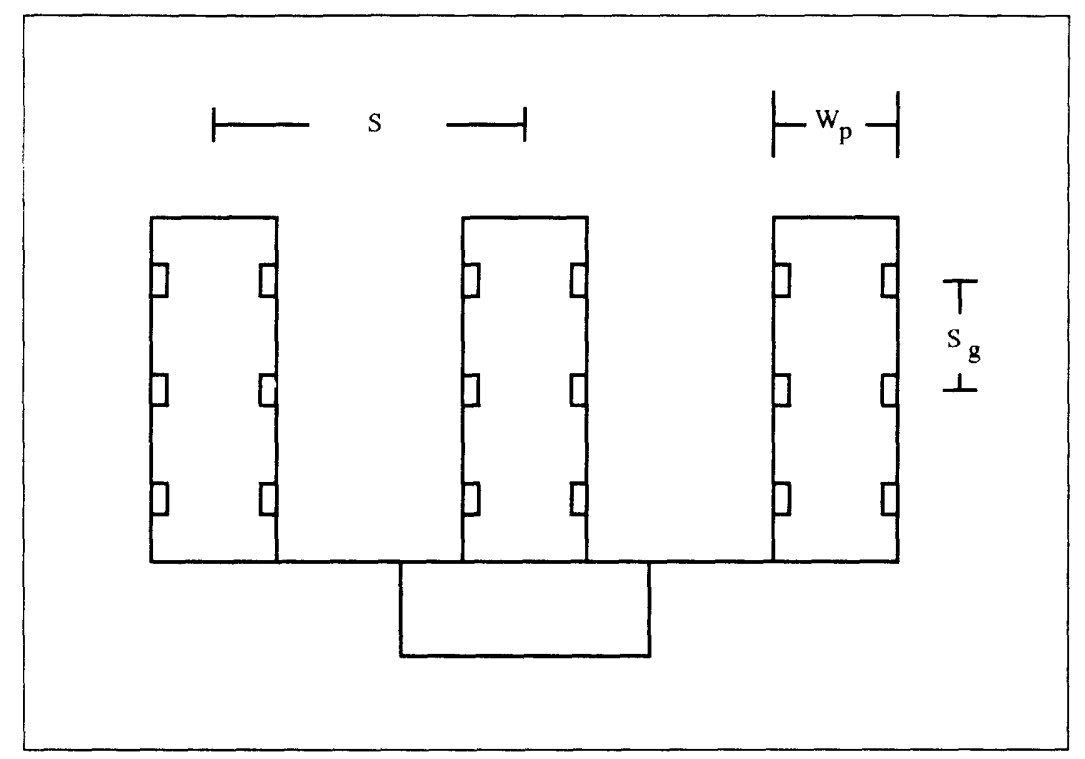

Figure 4.2: Centralized Parallel Pier Concept

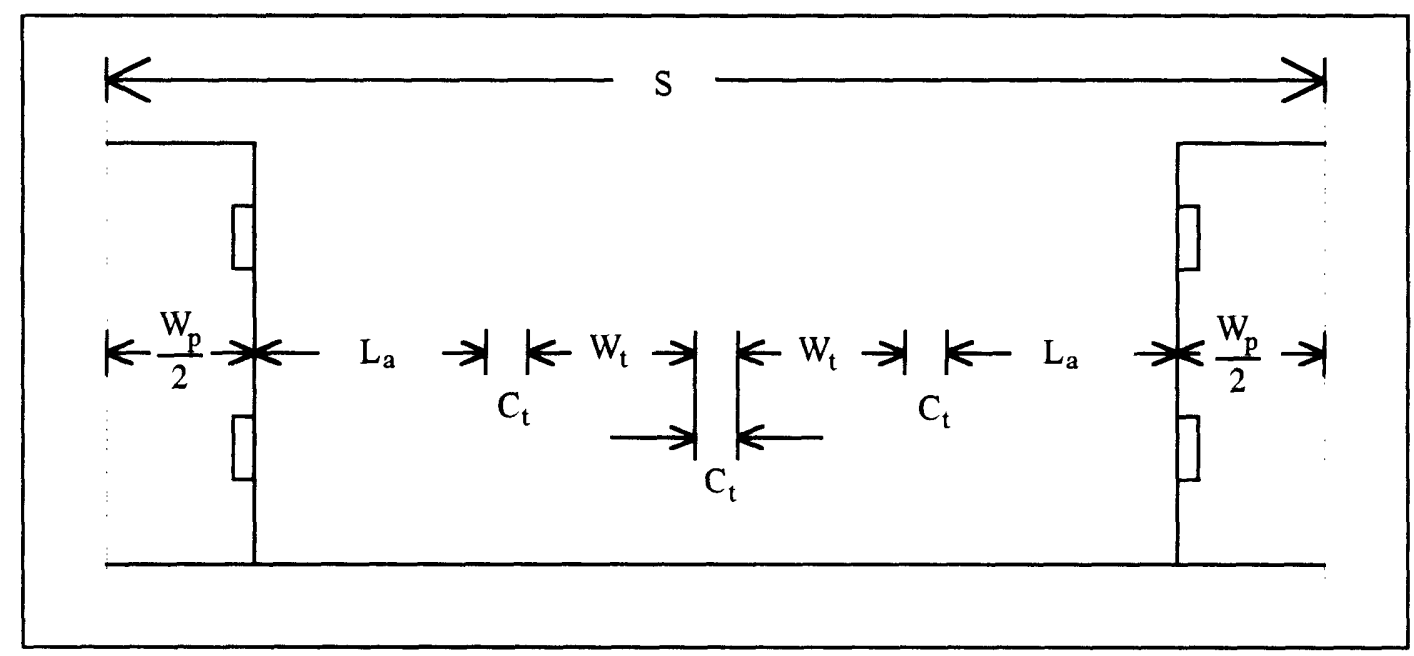

Figure 4.3: Possible Definition of $S$ 
$B_{C}=$ length of connector between terminal block and circular satellite concourses

$B_{R}=$ length of connector between terminal block and rectangular satellite concourses

$B_{T}=$ length of connector between terminal block and " $\mathrm{T}$ "-shaped satellite concourses

The length of $B$ depends on the number and geometry of the satellite concourses, as well as the physical limits of the landside acreage. Bandara defines the "angle of spread", $\beta$, as

$$
\begin{aligned}
& \beta=\text { maximum allowable angle subtended by the centerlines of the outermost } \\
& \text { satellite connectors (See Figure 4.5) }
\end{aligned}
$$

Given a value for the mandatory satellite separation, $S$, and the angle of spread, $\beta$, it is possible to calculate the minimum value of $B$ which maintains the necessary separation requirements. The following sections provide some examples of the calculations needed if the specific layout of the configuration is not provided (i.e. $B$ is not given).

\subsubsection{1 - Circular Satellites}

For circular satellites arranged radially about a centralized terminal block, connector lengths depend on both the number of satellites and the dimensions, i.e. the radius of each individual satellite (Figure 4.5). One possible method of estimating the minimum radius of a circular concourse (containing $g$ gates) is to inscribe a regular polygon having $g$ sides within the satellite. The interior angle of one triangle in the polygon, denoted by $2 \phi$, is simply

$$
2 \phi=\frac{2 \pi}{g}
$$

The radius of each satellite, $R_{S}$, can then be approximately

$$
R_{S}=\frac{S_{g} \cot \phi}{2}
$$




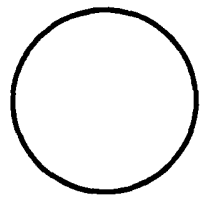

(a) Circular

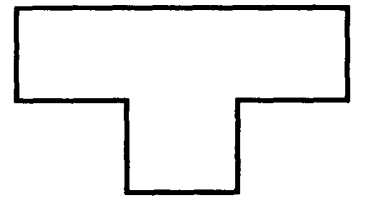

(c) "T"-Shaped

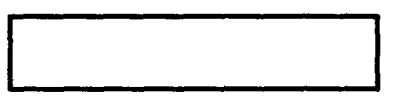

(b) Rectangular

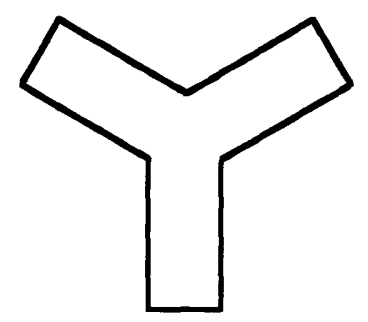

(d) "Y"-Shaped

Figure 4.4: Types of Satellites

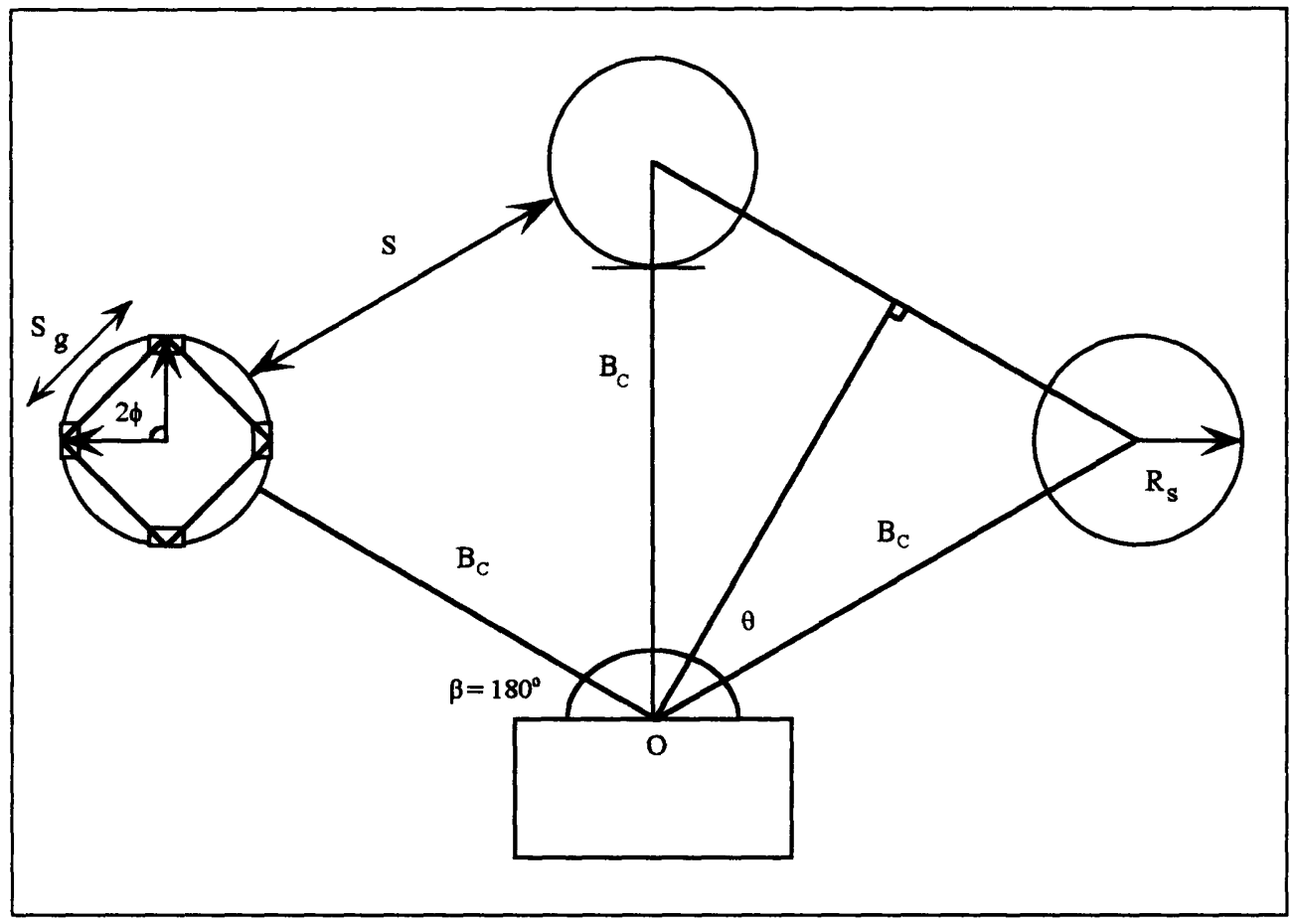

Figure 4.5: Centralized Satellite Configuration with Three Circular Concourses 
Note that for small values of $g$ this expression may overestimate the need for $R_{S}-$ other constructions are possible which account for the increased aircraft frontage afforded circular satellites. The minimum angle between any two concourses, $2 \theta$, is

$$
2 \theta=\frac{\beta}{(n-1)}
$$

where

$$
\begin{aligned}
& 2 \theta=\text { minimum angle subtended by the centerlines of adjacent connectors } \\
& \beta=\text { maximum "angle of spread" } \\
& n=\text { number of satellite concourses }
\end{aligned}
$$

Note that $2 \theta$ can be larger than the minimum value so long as spacing requirements are met. From the construction in Figure 4.5,

$$
\left(B_{C}+R_{S}\right) \sin \theta=\frac{S}{2}+R_{S}
$$

and thus

$$
B_{C}=\frac{S+2 R_{S}}{2 \sin \theta}-R_{S}
$$

We can use the values obtained from the above equations to derive the distances of the individual paths walked by passengers within centralized circular satellite configurations. The following section derives similar expressions for rectangular and "T"-shaped satellites.

\subsubsection{2 - Rectangular and "T"-Shaped Satellites}

For rectangular and "T"-shaped satellites (Figure 4.6), the lengths of the underground connectors ( $B_{R}$ and $B_{T}$, respectively) depend both on the length of each satellite and the angle subtended by any two adjacent connectors, $2 \theta$. One possible construction used to determine the length of a connector, $B_{R}$, yields 


$$
B_{R}=\frac{S+2 x}{2 \sin \theta} \quad \text { (4.7) } \quad \text { and } \quad x=\frac{L_{R}}{2} \cos \theta
$$

where

$$
\begin{aligned}
& B_{R}=\text { length of connector between terminal block and rectangular satellite concourses } \\
& 2 \theta=\text { minimum angle subtended by the centerlines of adjacent connectors } \\
& L_{R}=\text { length of the rectangular satellite } \\
& x=\text { intermediate value determined by construction }
\end{aligned}
$$

For "T"-shaped satellites, the length of $B_{T}$ can be calculated using expressions similar to Equations 4.7 and 4.8. Walking distances for individual passenger paths can then be determined based on the values obtained from these expressions.

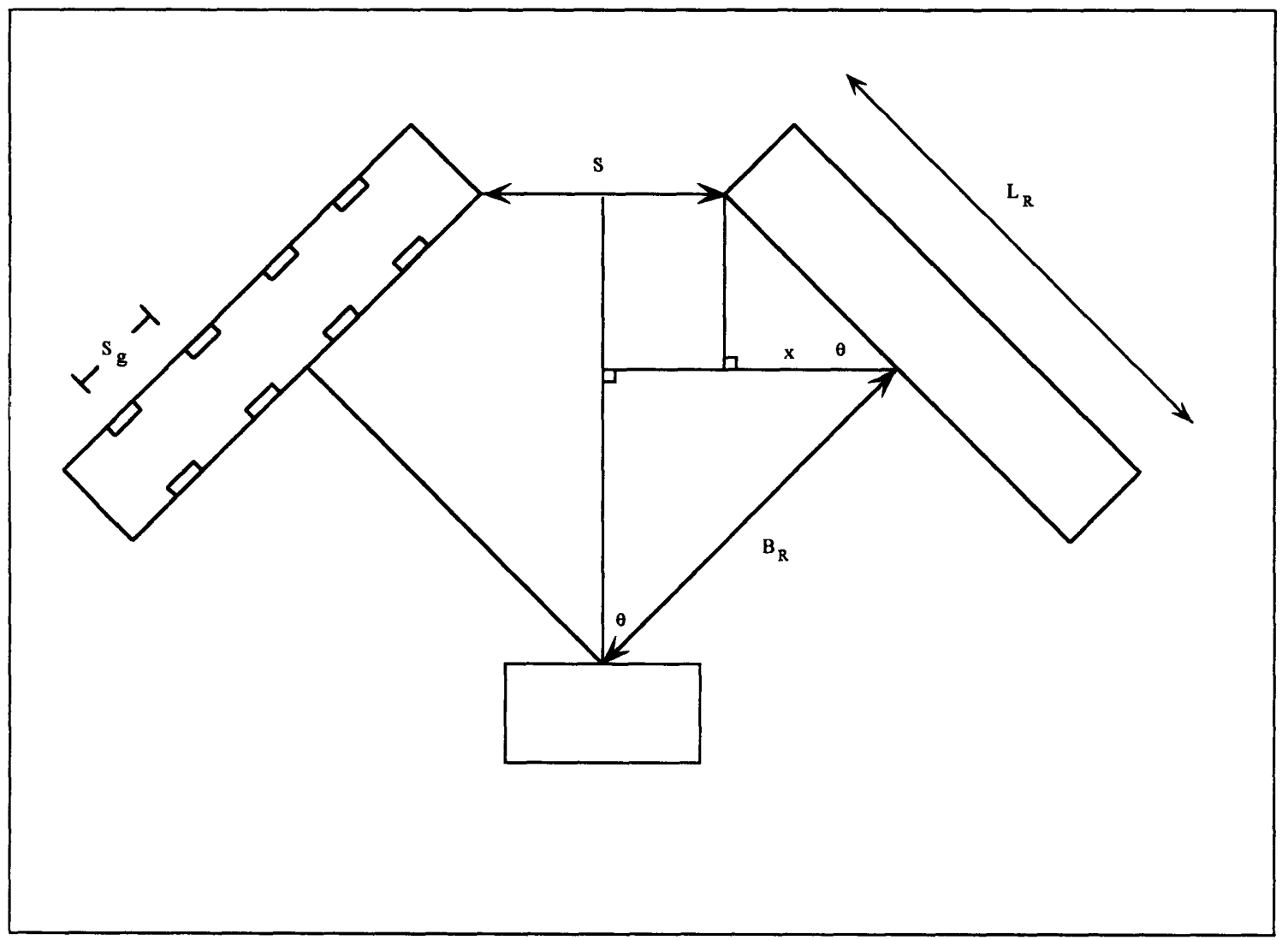

Figure 4.6 : Centralized Satellite Configuration with Two Rectangular Concourses 


\subsection{4 - The Midfield Concept}

Centralized midfield configurations can be represented geometrically in a similar fashion to satellite concepts. Originating and terminating passengers are assumed to arrive and depart through a single terminal block and travel to and from concourses "midfield" which are separated from the terminal block by some mandatory spacing, $B$. Direct transfer passengers can remain at midfield, but indirect transfers may need to return to the terminal block for intermediate services. The following sections suggest geometric representations of two possible midfield configurations, namely parallel linear and midfield "X" concepts.

\subsubsection{1 - Parallel Linear}

The parallel linear concept is similar to the parallel pier concept in its characterization (Figure 4.7). The departure concourse closest to the terminal block is spaced a distance $B_{P L}$ away, and all subsequent concourses are arranged in a parallel sequence separated by a distance $S$, which can be calculated using an expression similar to Equation 4.1.

\subsubsection{2 - Midfield " $\mathrm{X}$}

It is possible to characterize a midfield " $\mathrm{X}$ " configuration as an " $\mathrm{X}$ "-shaped satellite. Unlike previous characterizations, however, the mandatory spacing between the terminal block and the concourse, $B_{X}$, is not necessarily equal to the length of the connector element, $C_{X}$. If we assume that the connector ends at the center of the " $\mathrm{X}$ ", it can be shown (using the Triangle Inequality) that the position which minimizes the length of $C_{X}$ is such that the angle between the two closest arms is bisected by a line perpendicular to the terminal block (Figure 4.8). 
The length of the connector element, $C_{X}$, is then

$$
C_{X}=x+y \quad(4.9), \quad x=\frac{L_{X}}{2 \sqrt{2}} \quad(4.10), \quad \text { and } \quad y=\sqrt{B_{X}{ }^{2}-x^{2}}
$$

where

$C_{X}=$ length of connector between terminal block and midfield "X" concourse

$B_{X}=$ mandatory separation between terminal block and midfield " $\mathrm{X}$ " concourse

$L_{X}=$ length of one arm of midfield " $X$ " concourse

$x, y=$ intermediate values determined by construction

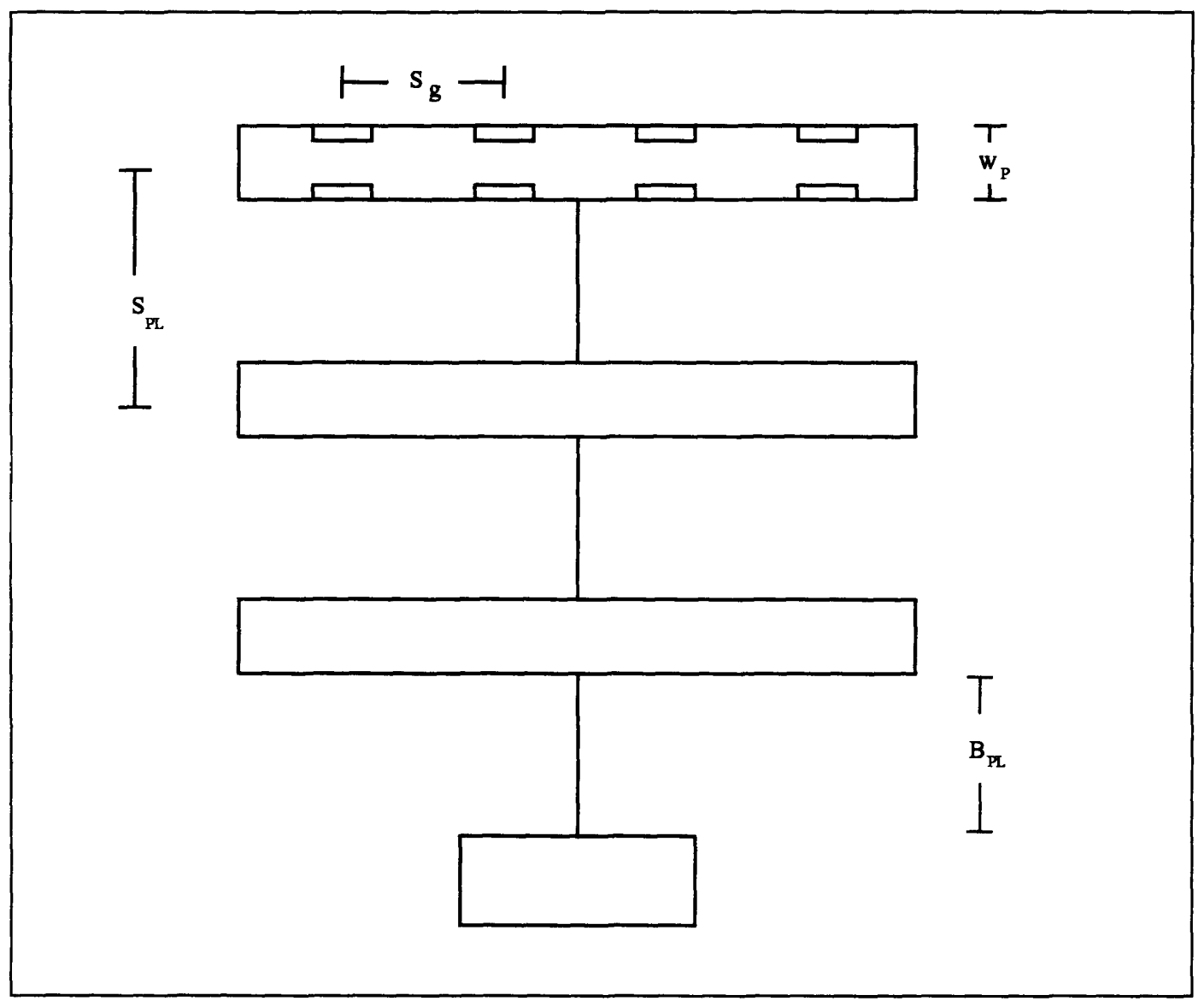

Figure 4.7 : Parallel Linear Midfield Concept 


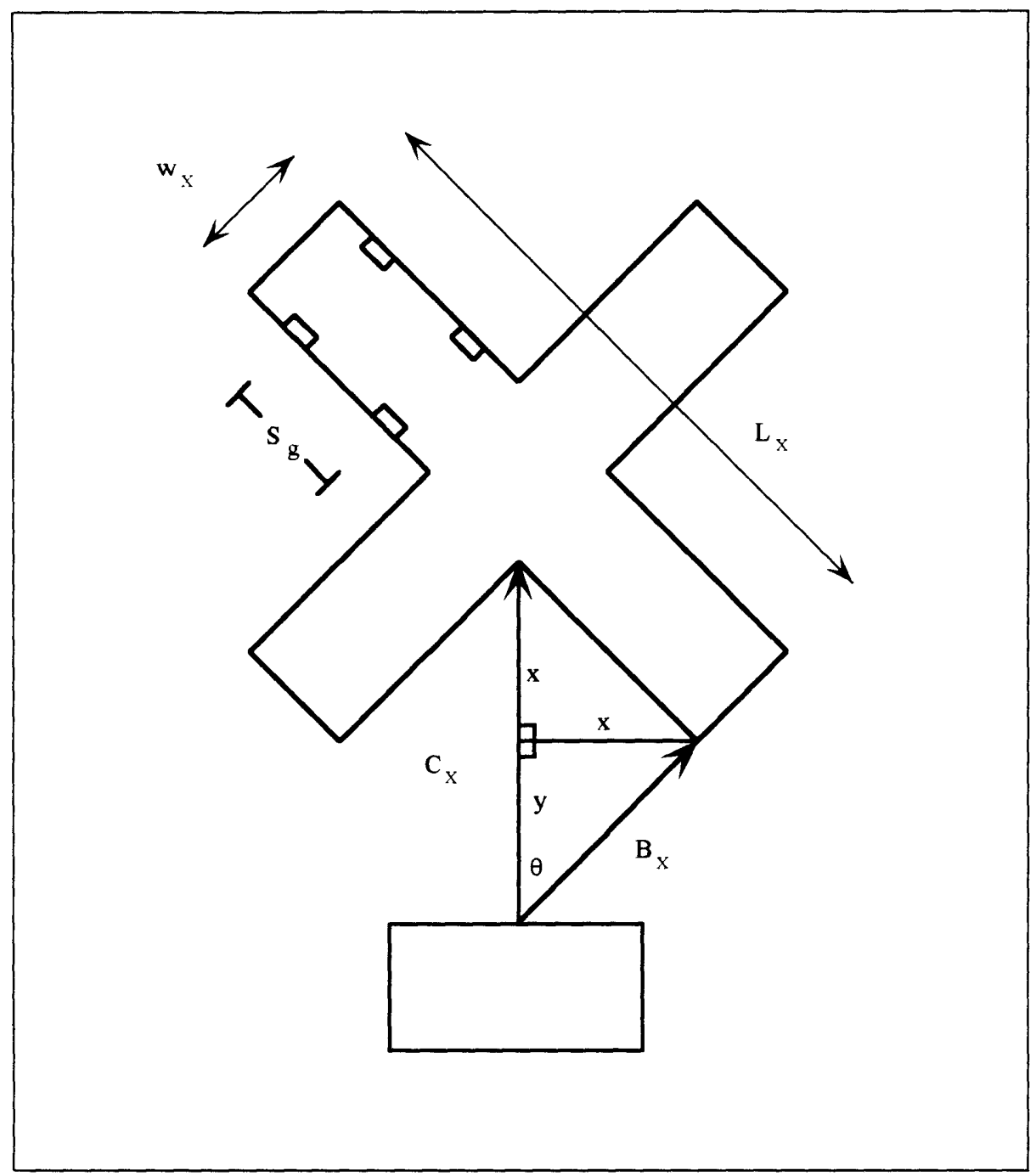

Figure 4.8: Midfield "X" Concept 


\section{2 - Decentralized Terminals}

Decentralized configurations differ from their centralized counterparts in that each concourse has a dedicated terminal block with duplicate facilities for check-in, baggage, and other auxiliary services. The following section describes possible geometric representations of several standard decentralized passenger building configurations.

\subsection{1 - The Gate Arrival Concept}

Unlike the centralized gate arrival configuration, the decentralized concept provides originating passengers with a direct interface between access/egress points and aircraft. Figure 4.9 illustrates one possible representation of a two unit gate arrival terminal configuration.

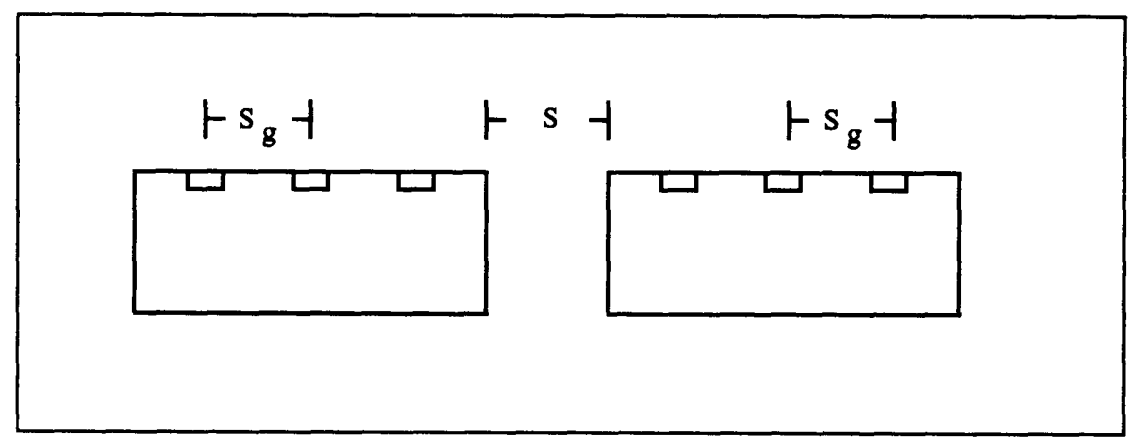

Figure 4.9: Decentralized Gate Arrival Concept

The length of each terminal block is governed by the number of aircraft stands. If we use Bandara's simplified method for determining distances, then originating and terminating passengers can again be assumed to walk a distance $b_{p}$ within the terminal block, where in this case $b_{p}$ for a decentralized terminal is assumed to be less than $b_{p}$ for a centralized configuration. Unit terminals are separated by some distance $S$, which does not necessarily depend on aircraft size. With these values and the globally defined parameters defined in Section 4.1, it is possible to determine the distances associated with individual passenger paths (for this particular construction). 


\subsection{2 - The Parallel Pier Concept}

The decentralized parallel pier configuration (Figure 4.10) is similar to the centralized configuration, but now each of the piers has its own terminal block. In this particular representation, piers are separated by a mandatory spacing requirement of $S$, which is either given or could be determined by an expression similar to Equation 4.1 for the centralized parallel pier concept. Walking distances can be determined using assumptions similar to those for the centralized parallel pier concept.

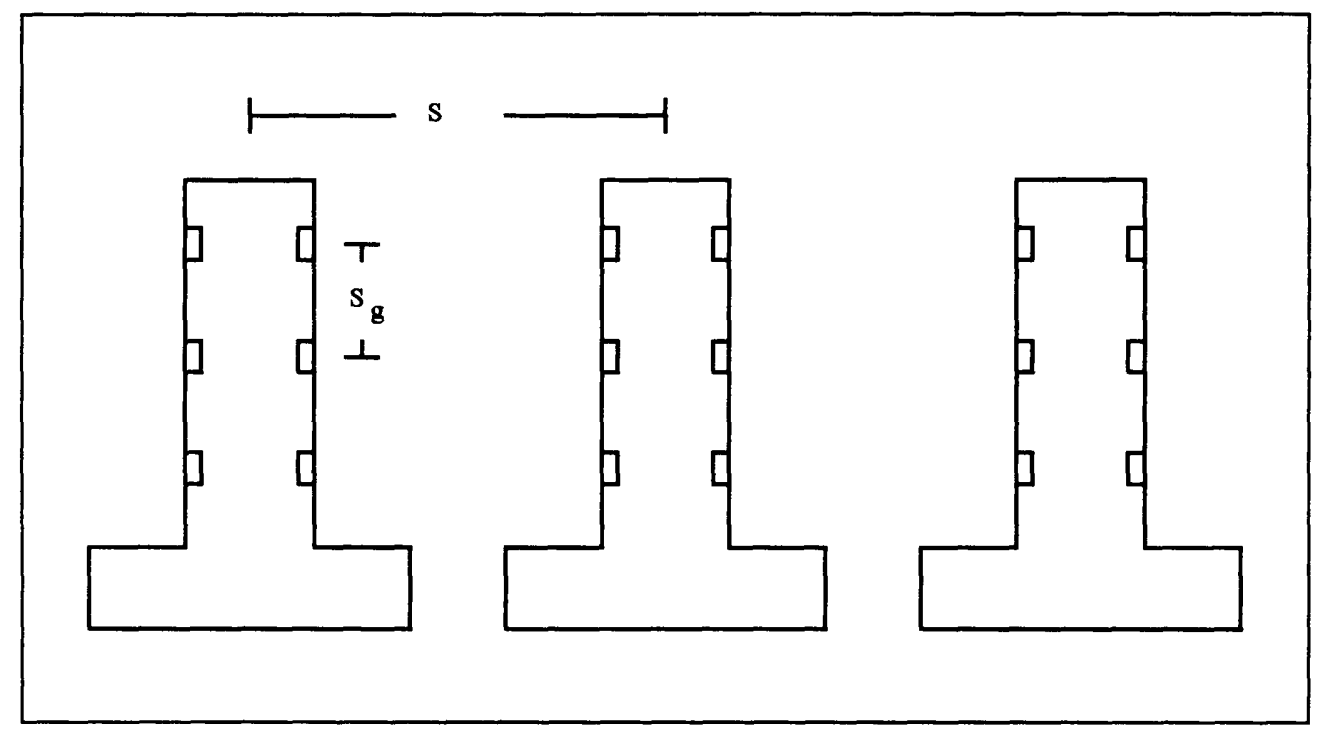

Figure 4.10: Decentralized Parallel Pier Concept

\subsection{3 - The Satellite Concept}

One possible construction for decentralized satellite configurations is to arrange the satellites radially about the intersection of the extended connector centerlines [Bandara 1989]. Individual terminal blocks can then be arranged in a semicircular fashion along each of the connector lines, separated from their respective satellite concourse by some distance, denoted by $B$. In Bandara's construction, he holds $B$ 
constant and maintains the mandatory spacing between satellite concourses, $S$, by varying the spacing between unit terminal blocks, denoted by $S$ (with an appropriate subscript). Thus,

$S_{\mathrm{C}}=$ distance between adjacent terminal blocks for circular satellite constructions

$S_{R}=$ distance between adjacent terminal blocks for circular satellite constructions

$S_{T}=$ distance between adjacent terminal blocks for circular satellite constructions

The following section describes possible geometric representations of decentralized satellite concepts.

\subsubsection{1 - Circular Satellites}

Figure 4.11 illustrates one possible geometric representation of a decentralized satellite configuration with three circular shaped concourses, according to Bandara's construction.

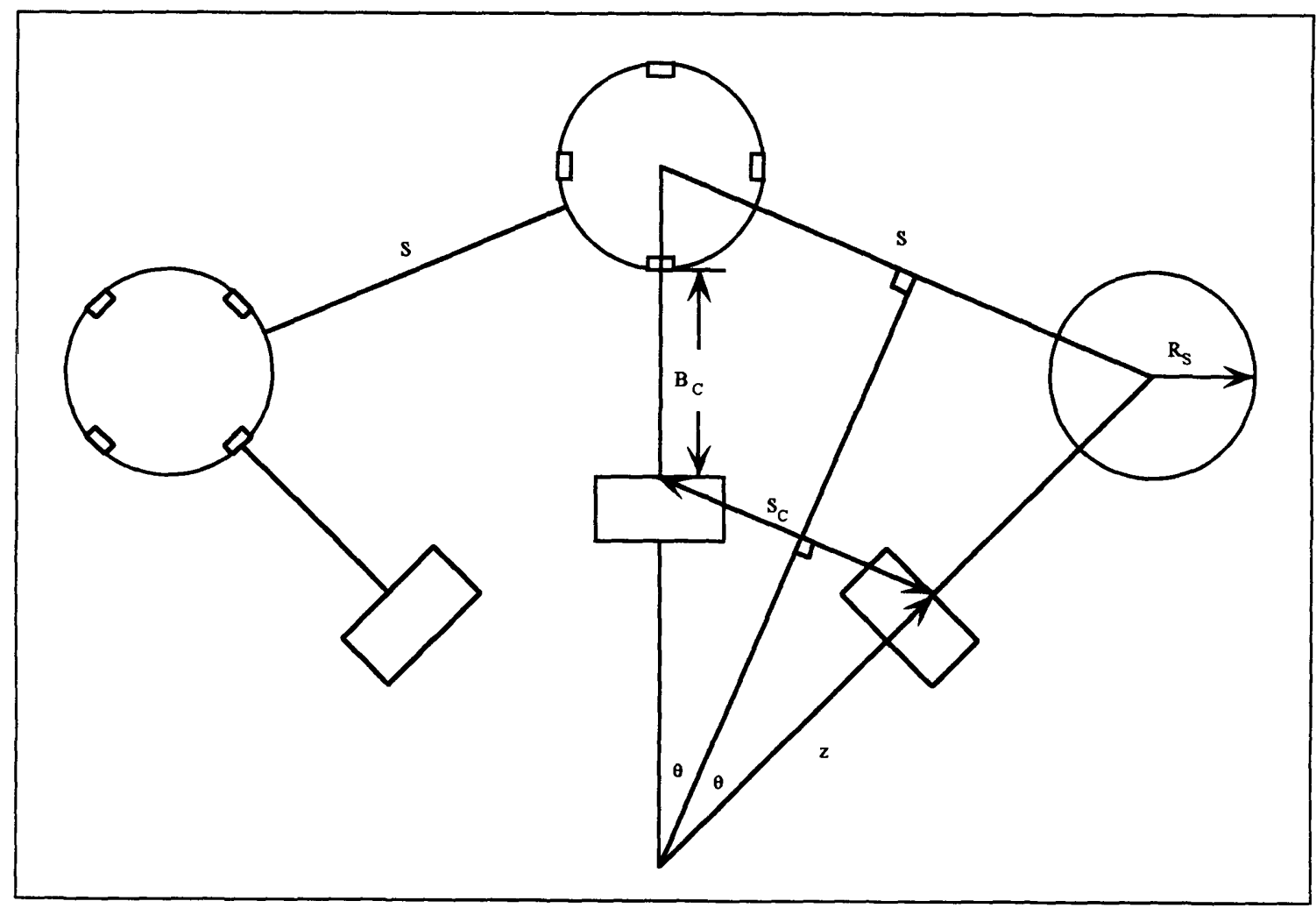

Figure 4.11: Decentralized Satellite Construction with Three Circular Concourses 
Bandara [1989] defines $\mathrm{S}_{\mathrm{C}}$ as :

$$
S_{C}= \begin{cases}2 z \sin \theta & \text { if } z>0 \\ 0 & \text { if } z=0\end{cases}
$$

where

$$
z=\frac{S+2 R_{S}}{2 \sin \theta}-\left(B_{C}+R_{S}\right)
$$

and

$S_{C}=$ distance between adjacent terminal blocks for circular satellite constructions

$S=$ mandatory spacing between satellites

$R_{S}=$ radius of circular satellite concourse

$B_{C}=$ mandatory spacing between terminal block and circular satellite concourse

$2 \theta=$ angle subtended by the centerlines of adjacent connectors

$z=$ intermediate value, by construction

Walking distances for individual passenger paths can then be determined using the above calculated values.

\subsubsection{2 - Rectangular and "T"-Shaped Satellites}

Figure 4.12 shows a possible representation of a decentralized satellite configuration with two "T"-shaped concourses. The calculations necessary to obtain values for $S_{R}\left(S_{T}\right)$ are similar to that for circular satellites. The necessary spacing between unit terminal blocks, $S_{T}$, is again

$$
S_{T}=2 z \sin \theta
$$

where

$$
z=\frac{S+2 x}{2 \sin \theta}-B_{T}
$$

and

$$
x=\frac{L_{T}}{2} \cos \theta
$$


and

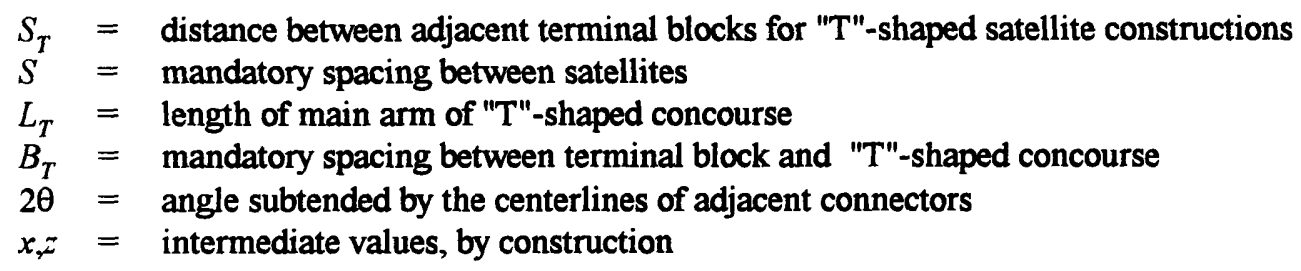

For rectangular concourses, the value of $L_{T}$ is replaced with $L_{R}$.

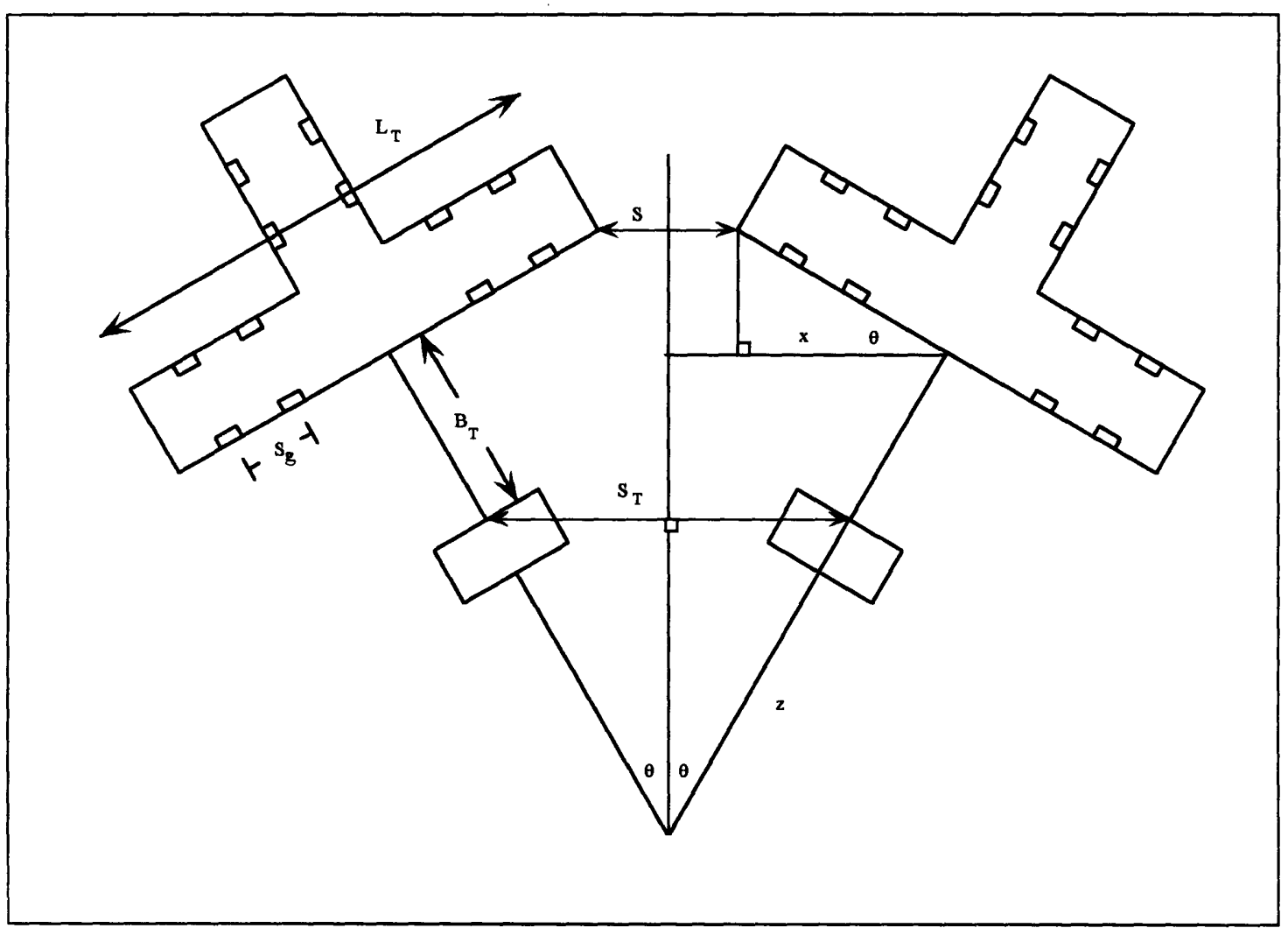

Figure 4.12: Decentralized Satellite Construction with Two "T"-Shaped Concourses 


\subsection{4 - The Midfield Concept}

Decentralized midfield concepts have multiple access points through which passengers can be taken midfield. Geometrically, decentralized midfield concepts can be constructed similarly to the decentralized satellite configurations described previously. Figure 4.13 shows one possible geometric representation of a decentralized, two-" $\mathrm{X}$ " midfield configuration.

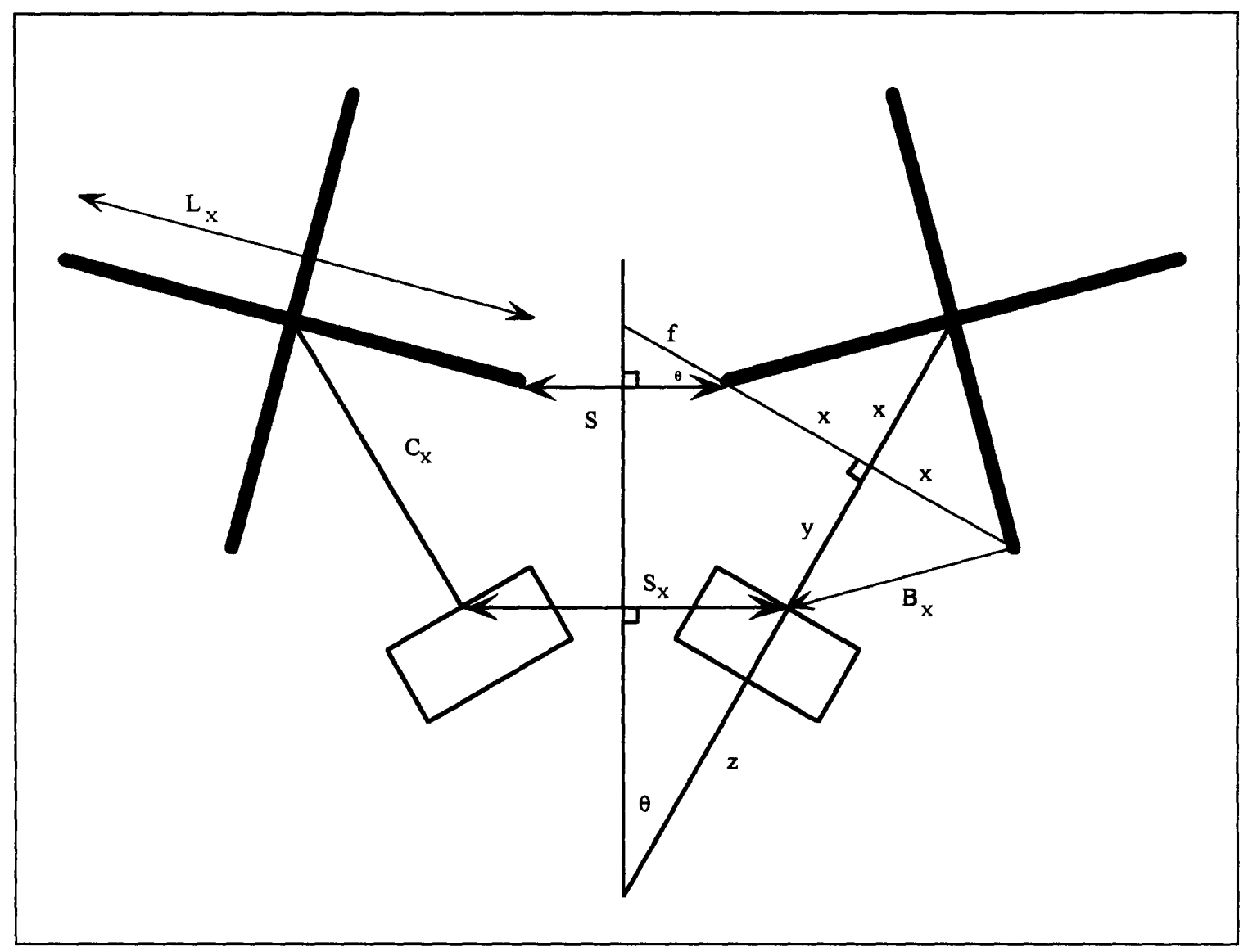

Figure 4.13 : Decentralized Midfield Configuration with Two "X" Concourses 
In this case, the value of $S_{X}$ can be determined as follows:

$$
S_{X}=2 z \sin \theta
$$

where

$$
\begin{array}{ll}
z=[(x+f) \cot \theta]-y & x=\frac{L_{X}}{2 \sqrt{2}} \\
y=\sqrt{B_{X}^{2}-x^{2}} & \text { (4.20) }
\end{array}
$$

and

$$
\begin{aligned}
& S_{X}=\text { distance between adjacent terminal blocks for midfield "X" constructions } \\
& S=\text { mandatory spacing between satellites } \\
& L_{X}=\text { length of one arm of "X" concourse } \\
& B_{X}=\text { mandatory spacing between terminal block and "X" concourse } \\
& 2 \theta \quad=\text { angle subtended by the centerlines of adjacent connectors } \\
& x_{2}, Z_{f}=\text { intermediate values, by construction }
\end{aligned}
$$

Tables 4.1 and 4.2 summarize the parameters used to construct the geometric representations of the centralized and decentralized passenger building configurations presented in this chapter. Note that the constructions we have presented are not unique -- they are presented to demonstrate techniques which can aid in standardizing the characterization of passenger building concepts. From these representations we can determine the absolute distances passengers will walk based on certain assumptions regarding the specific paths they follow. In the next chapter we therefore treat distance information as given, and demonstrate how to obtain estimates for potential configuration performance as measured by metrics such as expected walking distances and overall travel times. 
Table 4.1 - Parameters Used in Representing Centralized Configurations

\begin{tabular}{|c|c|}
\hline Parameter & Description \\
\hline Global & \\
\hline$G$ & Total number of gates in the airport \\
\hline$n$ & Number of individual concourses \\
\hline$g$ & Number of gates within a concourse \\
\hline$S_{g}$ & Gate spacing \\
\hline$b_{p}$ & Distance walked by within terminal block \\
\hline \multicolumn{2}{|l|}{ Pier } \\
\hline$S$ & Spacing between parallel pier concourses \\
\hline \multicolumn{2}{|l|}{ Satellite } \\
\hline$S$ & Spacing between satellite concourses \\
\hline $2 \theta$ & Minimum angle between adjacent satellite connectors \\
\hline$\beta$ & Maximum "angle of spread" for satellites \\
\hline$R_{S}$ & Radius of circular satellite concourse \\
\hline$B_{C}$ & $\begin{array}{l}\text { Spacing between terminal block and circular } \\
\text { satellite concourse }\end{array}$ \\
\hline$B_{R}$ & $\begin{array}{l}\text { Spacing between terminal block and rectangular satellite } \\
\text { concourse }\end{array}$ \\
\hline$B_{T}$ & $\begin{array}{l}\text { Spacing between terminal block and } \\
\text { "T"-shaped satellite concourse }\end{array}$ \\
\hline$L_{R}$ & Length of rectangular satellite concourse \\
\hline$L_{T}$ & Length of main arm of "T"-shaped satellite concourse \\
\hline
\end{tabular}

$\begin{array}{ll}\begin{array}{l}\text { Midfield } \\ S_{P L}\end{array} & \begin{array}{l}\text { Spacing between linear midfield concourses } \\ B_{P L}\end{array} \\ & \begin{array}{l}\text { Spacing between terminal block and first } \\ \text { parallel linear midfield concourse }\end{array} \\ S & \text { Spacing between midfield "X" concourses } \\ L_{X} & \text { Length of one arm of "X"-shaped concourse } \\ B_{X} & \begin{array}{l}\text { Spacing between terminal block and "X"-shaped } \\ \text { midfield concourse }\end{array} \\ C_{X} & \begin{array}{l}\text { Length of connector to midfield concourse imposed by } \\ \text { spacing } \mathrm{B}_{\mathrm{X}}\end{array}\end{array}$


Table 4.2 - Additional Parameters Used in Representing Decentralized Configurations

\section{Parameter Description}

Gate Arrival

$S$

Distance walked within terminal block

Satellite

$S_{C}$

Spacing between adjacent terminal blocks in circular satellite configurations

$S_{R}$ Spacing between adjacent terminal blocks in rectangular satellite configurations

$S_{T} \quad$ Spacing between adjacent terminal blocks in "T"-shaped satellite configurations

$S_{X} \quad$ Spacing between adjacent terminal blocks in midfield " $\mathrm{X}$ " configurations 


\title{
Chapter 5
}

\section{Estimating Potential Airport Performance}

\section{Part I : Passenger Convenience Measures}

\author{
Introduction
}

Once a geometric characterization of different configuration concepts is developed, it is possible to estimate various measures of potential performance. This chapter describes how a series of simple equations can be used to compute several such measures based on various assumptions regarding the distribution of passengers resulting from various airport operations. The techniques used to represent the airport environment are adapted from so-called object-oriented problem solving methods. Object-oriented computer languages (such as $\mathrm{C}++, \mathrm{LISP}$, and AP/L) organize large amounts of data into structures, or "objects", which can be operated on very efficiently. Section 5.1 describes how one might represent the airport environment within an object-oriented framework. Sections 5.2 and 5.3 introduce our method for estimating potential configuration performance as measured by passenger walking distances. Section 5.4 describes how similar techniques can be used to measure overall travel times, and Section 5.5 demonstrates how previously calculated data can be used to predict areas of potential congestion. Finally, 
in Section 5.6 we present a series of empirical examples to demonstrate how the models we introduce can be implemented on a given configuration.

\section{1 - Representing the Airport Environment}

Consider the two-terminal airport configuration illustrated in Figure 5.1. Terminal 1 contains a finger-pier concourse with three gates located along its perimeter. Terminal 2 contains a circular satellite concourse with four gates. Access to the circular satellite is through a below-grade connector which begins at the far side of the passenger processing facilities in Terminal 2, and ends in the center of the satellite concourse. No connector exists between the satellite concourse and the finger-pier concourse. By overlaying a Cartesian coordinate grid, we can determine the $x$ - and $y$-positions of all points in the airport, as well as the absolute distances between them.

Figure 5.2 depicts one possible object-oriented representation of the airport environment. At the highest level of description is the airport object, which is made up of passengers, passenger buildings, and aircraft. Passenger buildings are described by the number and location of the aircraft stands (gates) they contain. The passenger mix object is described in greater detail by individual objects for each passenger category considered. Table 5.1 illustrates a list of possible object attributes that can be used with this representation to describe the two-terminal airport depicted in Figure 5.1.

\section{Passenger Building Objects}

Each passenger building can be described by a list of attributes which uniquely identify it within the airport environment. Along with simple descriptive attributes (such as the building number and type), the object contains information regarding the number of gates and the locations of the entrance and waypoint (a location through which passengers must pass when transferring from one building to another) 


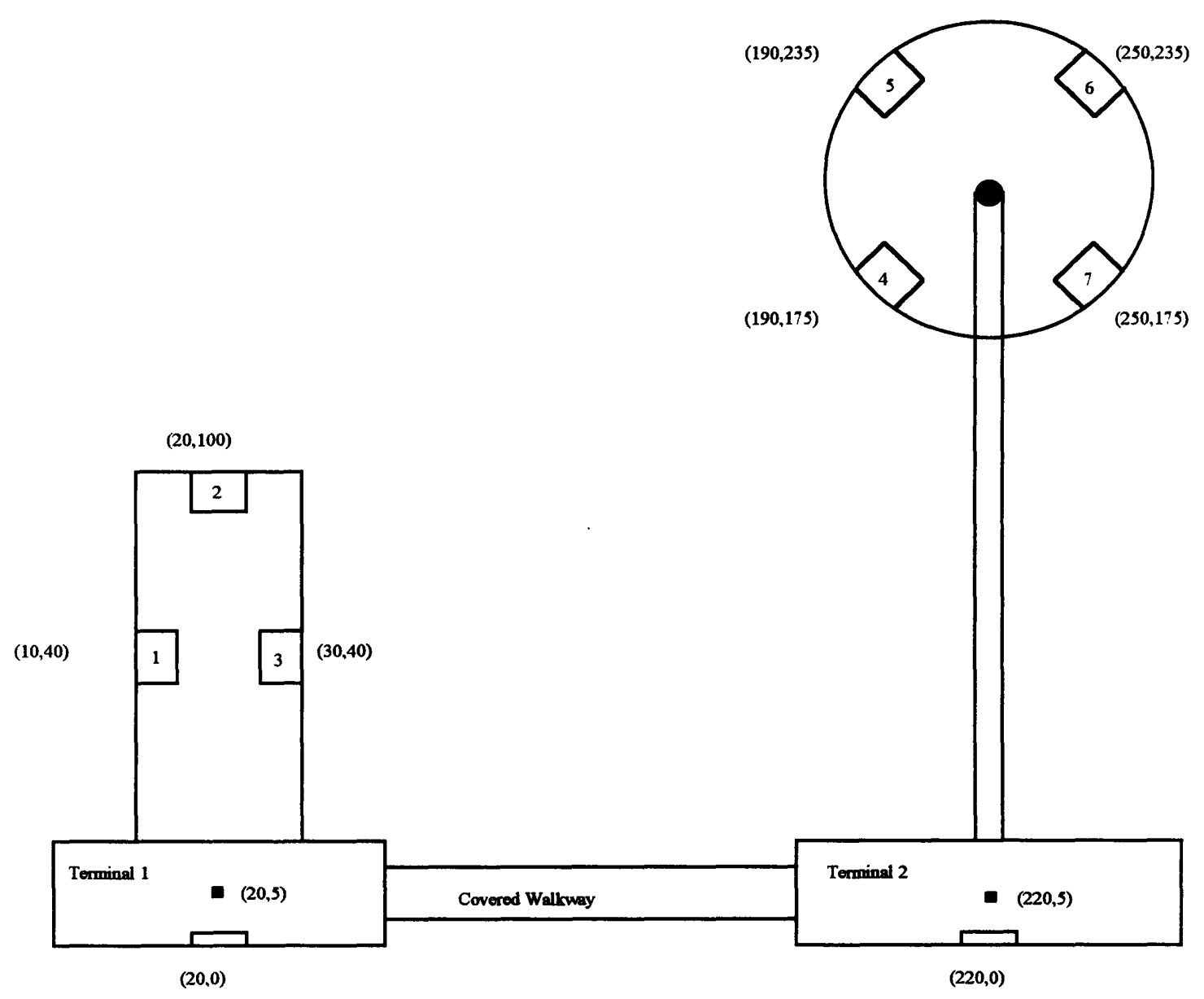

Figure 5.1 - Example of Two-Terminal Airport

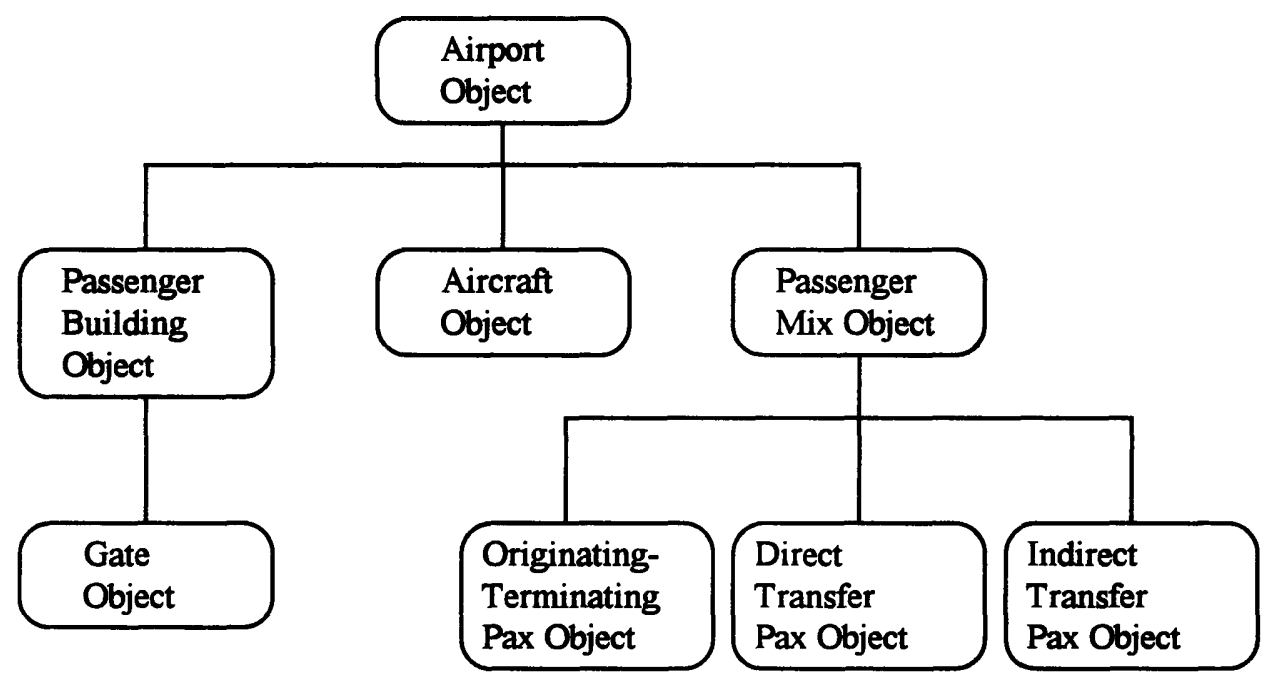

Figure 5.2: Possible Object-Oriented Representation of Airport Environment 
Table 5.1: List of Objects and Attributes Used to Represent the Airport Environment

Attribute List

Passenger Building Object

Terminal Number

Number of Gates

Type

Entrance Location

Waypoint Location

Intraterminal Transitions

Gate Object

Gate Number

Terminal Number

Position

Distance to Entrance

Distance to Waypoint

Capacity

Use

Aircraft Mix

Aircraft Separation Time

Utilization

Aircraft Object

Aircraft Type

Seating Capacity

Required Gate Size

Market

Average Load Factor

Turnaround Time

Passenger Mix Object

Demand Condition

Passenger Building

Originating Terminating

Direct Transfer

Indirect Transfer

Direct Transfer Passenger Object

Expected Walking Distance
Example

(Terminal 1)

1

3

Finger-Pier

$(20,0)$

$(20,5)$

Intelligent Scheduling

(Triangular Distribution)

(Gate 1)

1

1

$(10,40)$

$50 \mathrm{~m}$

$45 \mathrm{~m}$

Medium

All Demand Periods

$75 \%$ Medium Jets, $25 \%$ Small Jets

20 minutes

15 operations per day

(Boeing 737-300)

737-300

128 seats

Small

Shuttle

$67 \%$

$40 \mathrm{~min}$.

(Low Demand Conditions)

Low Demand

Terminal 1

$85 \%$

$10 \%$

$5 \%$

$145.3 \mathrm{~m}$ 
within the terminal. Finally, there is an attribute which describes the type of gate-assignment assumption made regarding intraterminal transitions (e.g. uniform assignment of aircraft to gates, intelligent scheduling, etc.).

Gate Objects

Gates within each passenger building are described by a list of attributes which include general location information as well as the specific $(x, y)$ coordinates of the gate on a Cartesian grid. Derived distance attributes are maintained in order to calculate distances quickly. A capacity attribute is used to describe the largest aircraft type that can be serviced at that gate. A parameter for demand use is also maintained to specify under what demand conditions (high season, peak hour, all, etc.) the gate is active. Finally, aircraft mix and separation data are maintained in order to calculate utilization estimates (measured in operations per unit time). The specific approach used to estimate utilization is discussed later in this chapter.

\section{Aircraft Objects}

Aircraft objects are generally identified by fleet type and seating capacity. Additionally, a more general size attribute is used to identify capacity restrictions and to calculate utilization rates maintained in the gate objects. Average load factor and turnaround time information are also maintained. Note that load factors are more generally associated with specific flights or at least city-pair markets, rather than aircraft. One of the main objections to previous models, however, is their reliance on very detailed information (such as actual flight schedules) for input data. It may be possible to use fleet types (with market group delimiters) as a surrogate for specific market information if we assume that, in general, certain fleet types are used in certain markets having characteristic load factors. Such an approximation may be preferable to a uniform load factor assumption implicit in models that do not recognize different aircraft types. 
Passenger Objects

The final set of objects used to describe the airport environment relate to passengers. Different demand conditions may have very different passenger mix profiles, and thus a separate passenger object may be used for each demand condition considered. Moreover, the mix may also vary by passenger building, depending on the concentration of individual carriers and the level of hub operations in place. Therefore, a separate object may be maintained for each building as well as each demand condition. The attributes required are simply a descriptor for identifying the specific demand condition (and passenger building) under consideration, and the proportion of total traffic made up by each passenger category. Unlike previous examples where attribute values were used to define individual elements uniquely, the attributes of the passenger mix object describe a "generic" passenger. Information specific to a particular passenger category (such as expected walking distances) is described by individual passenger category objects.

\section{2 - Estimating Passenger Walking Distances}

One often cited measure of passenger convenience used to assess the potential performance of an airport is that of passenger walking distances. Indeed, the geometry of the passenger building configuration greatly affects the distances passengers must walk. Issues such as space availability, waiting times at passenger processing facilities and security checkpoints, signage and the quality and price of concession merchandise are also important; however, the specific configuration of the passenger buildings has less of an impact on the level of service of these metrics. In the following section we introduce the basic model used to estimate overall passenger walking distances, and we show how to incorporate the effects of certain operational activities which have been neglected in previous research. 


\subsection{1 - Basic Model}

The model used to estimate the overall expected walking distance for a given configuration is simply a weighted average of the walking distance estimates for different passenger categories, weighted by the proportion of total traffic represented by each category. For a set of passenger categories $i(=1,2,3$, $\ldots, n)$, the overall expected walking distance for the configuration, $\mathrm{E}(D)$ or $\bar{D}$, can be expressed as

$$
\bar{D}=\sum_{i=1}^{n} p^{i} \bar{d}^{i}
$$

where

$$
\begin{aligned}
& \bar{D} \quad=\quad \text { Overall average walking distance for the configuration } \\
& p^{i} \quad=\quad \text { Proportion of traffic belonging to passenger category } i \\
& \bar{d}^{i} \quad=\quad \text { Walking distance estimate for passenger category } i
\end{aligned}
$$

Each of the individual walking distance estimates, $\bar{d}^{i}$, is in turn a weighted average of the absolute distances walked by each of the members belonging to passenger category $i$, weighted by the probability each path is walked. The probability each path is walked depends on assumptions made regarding the distribution of passengers among the gates at the airport. Below we discuss the three major passenger categories and some common assumptions regarding their paths.

\section{Passenger Types}

Passengers who begin and complete their journey at an airport are known as originating and terminating passengers, respectively. For the purposes of our analysis here, originating passengers are assumed to arrive at the airport entrance nearest to the terminal containing their departure gate. Their required walking distance, therefore, can be modeled as the distance between the appropriate terminal entrance and their departure gate. Since check-in facilities are generally located somewhere along this 
path (or in close proximity), no explicit distinction is made between the walking distances for passengers with advance seat assignments and those requiring check-in processing. Further, no distinction is made between walking distances for originating passengers carrying luggage and those who do not. This assumption is not meant to ignore the added inconvenience of walking with baggage. Separate estimates for the walking distances required with and without baggage can be maintained; however, to introduce the model, several simplifying assumptions are made in order to focus attention on the technique itself.

Similar to originating passengers, terminating passengers are assumed to depart the airport through the exit located nearest to their arrival terminal. Required walking beyond the exit is not necessarily affected by the configuration shape, and is thus not considered. Similar to check-in services, baggage claim services are often located along the path from the arrival gate to the terminal exit, and thus no distinction is made between terminating passengers with and without baggage. Thus the required walking distances for both originating and terminating passengers can be approximated by the distance between the departure (arrival) gate and the nearest terminal entrance (exit). Such an approximation helps in performing calculations quickly, though there is also an intuitive argument for its use. Separate estimates for originating and terminating passengers can be maintained if such a "reflexive" approximation is thought to be inappropriate for the given analysis.

Passengers who neither begin nor finish their journey at an airport are considered transfer passengers. Transfer passengers are required to travel from their arrival gate to their departure gate. The length and direction of the path depends on both the physical geometry of the terminal and whether the passenger is making a direct or indirect transfer. The more common type of transfer is a direct or hub transfer -- passengers going directly from their arrival to their departure gate. The required walking distance for a direct transfer can be approximated by the length of the most direct path between the respective gates, determined by the geometry of the terminal. Indirect or non-hub transfers, on the other hand, must include in their path some intermediate service point which is likely to increase the required walking distance. Most interline connections and international flights with domestic connections can be considered indirect transfers. 


\section{Originating and Terminating Passengers}

To estimate the expected walking distance for originating and terminating passengers, we employ a weighted average expression similar to Equation 5.1. In this case, we weight the absolute distances by the frequency each possible path is walked. The general expression for the expected distance of originating and terminating passengers, $\bar{d}^{o t}$, is

$$
\bar{d}^{o t}=\sum_{j=1}^{G} d_{e j}^{o t} p_{e j}^{o t}
$$

where

$$
\begin{array}{lll}
G & = & \text { total number of gates at the airport } \\
d_{e j}^{o t} & = & \text { absolute distance between nearest entrance } e \text { and gate } j \\
p_{e j}^{o t} & = & \text { probability of passenger traveling between entrance } e \text { and gate } j
\end{array}
$$

From the geometry of the terminal building it is possible to obtain the absolute distances walked between any two points in the airport, depending on the distance metric used. For the purposes of an example, we will employ a right-angle or Manhattan metric to express the distance walked between locations, i.e. for two points with $\mathrm{x}$ - and $\mathrm{y}$-coordinates $\left(x_{1}, y_{1}\right)$ and $\left(x_{2}, y_{2}\right)$, the distance between them (in the absence of barriers), $d_{12}$, is

$$
d_{12}=\left|x_{2}-x_{1}\right|+\left|y_{2}-y_{1}\right|
$$

To obtain the values for $p_{e j}$ in Equation 5.2 above, assumptions must be made regarding the distribution of originating and terminating passengers at each of the gates. We will explore different distribution assumptions in the next section. 


\section{Direct and Indirect Transfer Passengers}

We employ an expression similar to Equation 5.2 to estimate the expected walking distance for transfer passengers. The general expression for the expected walking distance of direct transfer passengers, $\bar{d}^{d t}$, is

$$
\bar{d}^{d t}=\sum_{i=1}^{G} \sum_{j=1}^{G} d_{i j}^{d t} p_{i j}^{d t}
$$

where

$$
\begin{array}{lll}
\bar{d}^{d t} & = & \text { expected walking distance for direct transfer passengers } \\
d_{i j}^{d t} & = & \text { absolute walking distance between gate } i \text { and gate } j \\
p_{i j}^{d t} & = & \text { unconditional probability of passenger transferring between gate } i \text { and gate } j \\
G & = & \text { total number of gates at the airport }
\end{array}
$$

We can also express the estimate in terms of a weighted average of conditional distances walked by passengers arriving at specific gates. For example, we can define the expected distance walked by direct transfer passengers arriving at Gate $i, \bar{d}_{i}^{d t}$, as :

$$
\bar{d}_{i}^{d t}=\sum_{j=1}^{G} d_{i j}^{d t} p_{j / i}^{d t}
$$

where

$$
\begin{array}{lll}
\bar{d}^{d t} & = & \text { expected walking distance for direct transfer passengers } \\
d_{i j}^{d t} & = & \text { absolute distance between gate } i \text { and gate } j \\
p_{j / i}^{d t} & = & \begin{array}{l}
\text { conditional probability of passengers departing from gate } j, \\
\text { given that they arrive at Gate } i
\end{array} \\
G & =\quad \begin{array}{l}
\text { total number of gates at the airport }
\end{array}
\end{array}
$$


The expression used to estimate expected walking distances for indirect transfer passengers is identical to Equation 5.5 above. The distances walked by indirect transfers, however, may be longer than those for direct transfers (arriving and departing from the same pair of gates), if the intermediate service point is not located directly along the path.

\section{Supplementary Statistics}

In addition to the expected value of the overall passenger walking distance, it is possible to estimate other statistics to describe more fully the entire walking distance distribution. Indeed, because of the level of detail with which the data could be maintained, we could produce the distribution itself simply by plotting the absolute distances of all possible paths along with their corresponding probabilities. We denote this probability distribution by $p_{d}\left(d_{o}\right)$, where

$$
p_{d}\left(d_{o}\right)=\text { probability that the value of the random variable } d \text { is } d_{0}
$$

Using this distribution we can calculate other supplementary statistics in order to compare the potential performance of different passenger building configurations.

Variance

In addition to the mean, we can also calculate the second moment (variance) of the walking distance distribution, $\sigma_{D}^{2}$, using

$$
\sigma_{D}^{2}=E\left(D^{2}\right)-[E(D)]^{2}
$$

where

$$
\begin{array}{lll}
\sigma_{D}^{2} & = & \text { variance (second central moment) of the walking distance } \\
\mathrm{E}\left(D^{2}\right) & = & \text { second moment of the walking distance } \\
\mathrm{E}(D)= & \text { first moment of the walking distance }
\end{array}
$$


Excess Statistics

Another set of statistics used to describe potential performance are measures of "excess". In the case of walking distances, we can define a threshold distance, $E_{d}$ beyond which walking distances are considered excessive. For a given walking distance distribution, we can define two additional statistics. The first is the probability that a randomly selected passenger will have to walk a distance greater than the excess threshold, $E_{d}$. For a given distribution, $p_{d}\left(d_{o}\right)$, the probability of excess walking, $\mathrm{P}\left[\mathrm{d}>E_{d}\right]$, is defined as:

$$
P\left[d>E_{d}\right]=\int_{d_{o}=E_{d}}^{\infty} p_{d}\left(d_{o}\right) d d_{o}
$$

The second statistic is the mean excess walking distance, $\bar{d}_{e}$, the conditional first moment of the walking distance distribution given that the distance threshold will be exceeded, or

$$
\bar{d}_{e}=\int_{d_{o}=E_{d}}^{\infty} d_{o} \cdot \frac{p_{d}\left(d_{o}\right)}{P\left[d>E_{d}\right]} d d_{o}
$$

From an airlines perspective, walking distances are often used as a surrogate for connection reliability -- the shorter the distance transfer passengers must walk, the more likely they are to make their connection. Small differences in the mean distance may not be as important as keeping, say, 99 percent of the distances transfer passengers must walk, below some threshold (such that they are almost certain to make their connection). 


\subsection{2 - Estimating Transition Probabilities}

The absolute walking distances used in the preceding expressions are uniquely determined by the geometry of the passenger building configuration, and can be obtained using the characterizations described in Chapter 4. The individual transition probabilities depend on assumptions made regarding the distribution of passengers to and from gates within the airport. Detailed, Monte-Carlo simulation programs use input flight schedules to obtain individual transition probabilities. Analytic formulae techniques, on the other hand, make simplifying assumptions regarding passenger distributions in order to obtain closed-form expressions for potential configuration performance. One of the most common assumptions made is the "uniform gate" assumption, i.e. that all gates within the airport have identical capacity and identical utilization, and thus have a uniform distribution of passengers [Bandara 1989], [Robusté 1991a,b], [Wirasinghe, et al. 1987, 1988]. To employ such an assumption in our model, the transition probability matrices for an airport with $G$ gates would simply be

$$
P^{o t}=\left[\begin{array}{lll}
\frac{1}{G} & \cdots & \frac{1}{G}
\end{array}\right] \quad(5.9) \quad P_{j / i}^{d t}=P_{j / i}^{i t}=\left[\begin{array}{ccc}
\frac{1}{G} & \ldots & \frac{1}{G} \\
\ldots & \frac{1}{G} & \ldots \\
\frac{1}{G} & \ldots & \frac{1}{G}
\end{array}\right]
$$

where row $i$ represents the passenger's arrival gate and column $j$ represents the departure gate. Such a uniformity assumption neglects the effects of operational realities and controls such as those described in Chapter 4. Using our object-oriented approach rather than a single equation, however, allows us to alter the transition probabilities using simple, fast calculations based on the level of information we wish to incorporate into the model. The following sections suggest some improvements upon the "uniform gate" assumption, and demonstrate how new transition probabilities could be calculated. 


\section{Intelligent Scheduling}

Dominance of individual carriers at a particular airport and effective stand management policies (flight-to-gate assignment procedures) are likely to influence the distribution of passengers within a given configuration. One result of such activities may be that connecting passengers will be more likely to depart from gates closer to their arrival gate. The following sections discuss possible ways to calculate walking distance estimates which account for such activities.

Interterminal Transitions

One possible way of capturing the effects of air carrier dominance at a particular airport is to introduce a transition matrix $\mathrm{X}$, whose elements, $x_{m n}$, represent the probability of passengers transferring from Terminal $m$ to Terminal $n$. The matrix X must obey the following condition :

$$
\sum_{n} \sum_{m} x_{m n} \sum_{j} \sum_{i} p_{j / i, m}=1
$$

The expression to estimate conditional walking distances for direct transfer passengers arriving at Gate $i$ located within Terminal $m$ then becomes

$$
\bar{d}_{i}^{d t}=\sum_{n=1}^{N}\left[x_{m n} \cdot \sum_{j=1}^{g_{n}} d_{i j}^{d t} p_{j / i, m}^{d t}\right]
$$

where :

$$
\begin{array}{lll}
\bar{d}^{d t} & =\quad \text { expected walking distance for direct transfer passengers } \\
x_{m n} & =\quad \text { probability of transferring between Terminal } m \text { and Terminal } n \\
d_{i j}^{d d} & =\quad \text { absolute walking distance between gate } i \text { and gate } j
\end{array}
$$


and

$$
\begin{array}{lll}
p_{j i \text { itm }}^{d t}= & \text { conditional probability of transferring to Gate } \mathrm{j} \text { given arrival } \\
N & =\begin{array}{l}
\text { at Gate } i \text { within Terminal } m \\
\text { total number of terminals at the airport }
\end{array} \\
g_{n}= & \text { number of gates located within Terminal } n
\end{array}
$$

\section{Flight-to-Gate Assignments}

For transfers within a specific concourse, the impacts of effective stand management can be captured with varying degrees of complexity. As mentioned in Chapter 4, one of many possible methods would be to model gate affinity as being inversely proportional to the distance to the arrival gate, i.e. to use a triangular rather than a uniform distribution to model intraterminal transitions. The probability of passengers departing from their own arrival gate (whose required walking distance is zero), can be obtained from empirical or forecast data. The expression that could then be used to approximate gate affinities for our simple intelligent scheduling model is

$$
p_{j / i, m}^{d t}=\left(1-\frac{d_{i j}^{d t}}{\sum_{j \neq i} d_{i j}^{d t}}\right)\left(1-p_{i / i}^{d t}\right) \forall j \in m, j \neq i
$$

\section{Aircraft Effects}

Another implicit result of the "uniform gate" assumption is that the volume of passengers

"witnessed" by every gate (per unit time) is identical, neglecting differences in capacity and utilization. Thus, differences in gate affinity would arise only from the desire of the airport operators or airlines to assign gates for connecting flights closer together. Differences in passenger volumes alter the distribution pattern of passengers arriving and departing from specific gates. We refer to the probability of a 
passenger arriving or departing from a gate based solely on the mix of aircraft serviced there as the gate's demand share.

The capacity of a gate is often expressed in terms of the largest aircraft that it can service -- gates able to accommodate large aircraft, for instance, also generally accommodate medium-sized and small aircraft. The breakdown of aircraft utilization at a gate is primarily determined by the gate assignment policy. A Large gate, for example, may serve 40 percent large aircraft, 50 percent medium-sized aircraft, and only 10 percent small aircraft. Given the gate use by aircraft type, two additional factors influence the demand rate : the expected number of passengers (per arrival or departure) and the gate occupancy time for each aircraft type. In general, larger aircraft carry more passengers, but have longer turnaround times. Conversely, smaller aircraft carry fewer passengers but can be turned around more quickly, thus allowing more operations per unit time.

One possible method to calculate a gate's demand share is to define a family of $n$ aircraft objects which have a list of attributes including capacity $C=\left(c_{1}, c_{2}, \ldots, c_{n}\right)$, average load factor $F=\left(\mathrm{f}_{1}, \mathrm{f}_{2}, \ldots, \mathrm{f}_{n}\right)$, and average gate occupancy times $A=\left(\mathrm{a}_{1}, \mathrm{a}_{2}, \ldots, \mathrm{a}_{n}\right)$, which include aircraft turnaround times and an estimate for the average separation time between aircraft arrivals. Gate objects would also have a list of attributes that include utilization by aircraft type, $u_{i j}$, expressed as a fraction. Thus, if Gate $i$ were a Large gate as described previously, the $u_{i j}$ vector would be :

$$
u_{i j}=\left[\begin{array}{lll}
.40 & .50 & .10
\end{array}\right]
$$

where the family of aircraft objects is defined as (Large, Medium, and Small).

The number of seats each gate will "witness" throughout a time period of $t$ minutes can be calculated using the attributes listed above. For Gate $i$, the number of seats, $S_{i}$, witnessed is

$$
S_{i}=\sum_{j=1}^{n} u_{i j}\left(c_{j} \cdot \mathrm{f}_{j}\right)\left(\frac{t}{a_{j}}\right)
$$


where :

$\begin{array}{lll}S_{i} & = & \text { total seats witnessed by Gate } i \\ n & = & \text { total number of aircraft types considered } \\ u_{i j} & = & \text { percentage utilization of aircraft type } j \text { at Gate } i \\ c_{j} & = & \text { capacity (in seats) of aircraft type } j \\ f_{j} & = & \text { average load factor of aircraft type } j \\ a_{j} & = & \text { average gate occupancy time of aircraft } j \\ t & = & \text { length of time period considered }\end{array}$

The demand share, $b_{i}$, for an individual gate is then

$$
b_{i}=\frac{S_{i}}{\sum_{i=1}^{G} S_{i}}
$$

where :

$$
\begin{array}{lll}
b_{i} & = & \text { demand share of Gate } i \\
S_{i} & = & \text { total seats witnessed by Gate } i \\
G & = & \text { total number of gates at the airport }
\end{array}
$$

To incorporate aircraft effects into our transition probabilities, we simply weight the gate affinities calculated previously by the demand shares calculated using Equations 5.14 and 5.15 above.

\section{Dynamic Gate Selection}

Varying levels of passenger demand place different requirements on an airport and its services throughout the day. Two typical passenger demand profiles faced by airport owners are shown in Figure 4.13. The first profile [4.13(a)] is characterized by an almost constant level of demand. The second profile [4.13(b)] is characterized by distinct peaks in the morning and evening. Airport operators facing the second demand profile can exploit such variability by using only a subset of gates during offpeak periods of demand. 


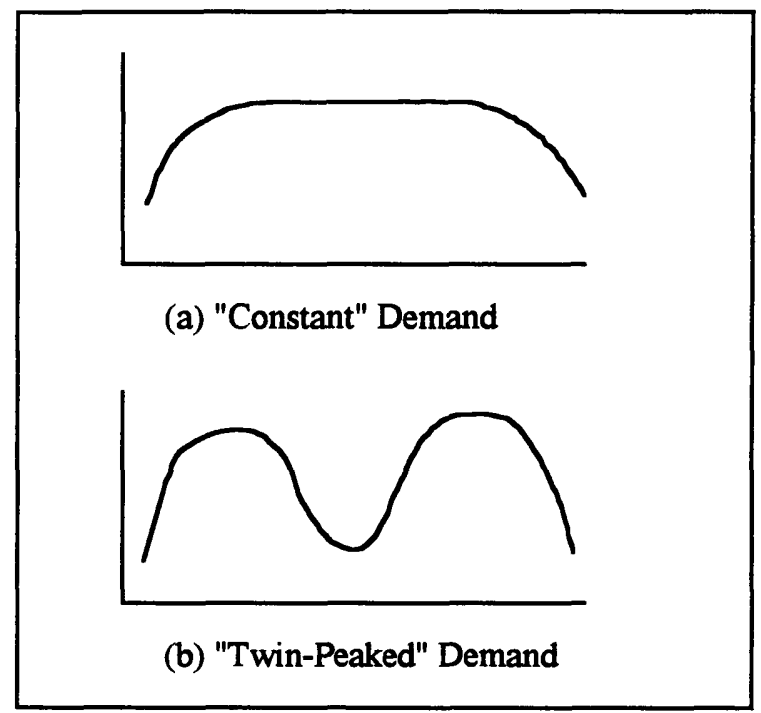

Figure 5.3: Possible Daily Demand Profiles

The ability to allocate gate usage dynamically on the basis of demand patterns can have significant impacts in expected walking distances. By using gates only in one terminal, for example, direct transfer walking distances are reduced by eliminating lengthy interterminal transitions. In Salt Lake City, Delta Air Lines dynamically reduces gate use along its piers in order to centralize operations and passenger flows during periods of low demand. Conversely, during periods of peak demand Zurich Airport will use remote aircraft parking spaces (unused during much of the day) in order to handle the increased demands on airport services.

To incorporate the effects of dynamic gate selection into our estimation of expected walking distances, a separate series of calculations is performed only on the set of gates used during high or low demand periods. The separate overall estimates can then be combined to obtain a single estimate, based on the fraction of time the airport operates under the different conditions. Note that it is the judicious selection of gates used during different demand conditions that can potentially reduce walking distances, not the variability in the demand pattern itself. 


\section{3 - Mandatory Walking Distances}

Steady growth in passenger traffic volumes and the concomitant use of larger, wide-body aircraft have resulted in the need for larger landside passenger buildings. The growing size of landside area facilities has naturally brought about an increase in passenger walking distances. To alleviate excessive walking distances, airport operators have introduced various "people-moving" systems to provide for short-haul collection and distribution of passengers [IATA 1989]. The LATA Airport Terminals Reference Manual cites two factors that should be considered when introducing a people-moving system into the terminal environment : (1) the elapsed time required to travel the necessary distance and (2) the personal effort required to cover the distance.

The manual suggests that for distances under 600 meters the consideration of effort is more important than the time factor. For distances under 600 meters, the actual time traveled may be greater than the time spent walking; however, the effort required, particularly if the passenger is carrying baggage, may be less if the mechanical device is used. Distances over 600 meters are considered excessive, and in those instances elapsed time becomes increasingly important. In fact, IATA suggests that when the distance between the point of check-in and the point at which passengers board the aircraft exceeds $300 \mathrm{~m}$, consideration should be given to providing a mechanical people-moving system.

The introduction of people-moving systems greatly affects the analysis of passenger building configurations in terms of overall passenger walking distances since passengers are no longer required to walk the entire distance dictated by the geometry of the configuration. As mentioned previously, the trade-off involved in using these mechanical means is often one of time versus convenience. Passengers electing to use people-moving systems reduce their overall walking distances, but may actually add to their overall travel times due to waiting and necessary deviations from their most direct path. The selection of the most appropriate configuration (based on measures of passenger convenience) should include the impacts of mechanical devices on the distances and times required to travel between points within the landside environment. The remainder of this section discusses the primary types of mechanical 
devices used as people-moving systems, and suggests a new measure of passenger convenience to be used in comparing terminal configurations, namely mandatory passenger walking distances.

\section{Moving Walkways}

Moving walkways, also known as moving sidewalks or horizontal passenger conveyors, are used to reduce walking distances within passenger buildings and between concourses that are joined by enclosed corridors. The effect of moving walkways on passenger convenience is to eliminate mandatory walking distances where such devices exist. Passengers who remain stationary on the conveyor eliminate walking distances entirely, while those who walk reduce the total distance due to increased speed relative to the ground. In either case, the required walking distance is less than the overall absolute distance. When modeling passenger convenience in terms of mandatory walking distances, segments containing moving walkways should either be eliminated or reduced by some distance which incorporates the speed of the passenger and of the conveyor.

\section{Passenger Rapid Transit Systems}

For transport between passenger buildings, two other types of people-moving systems exist. The first type is a simple system of shuttle buses which operate on existing roadways and which travel in designated "loops" within the airport environment. Different shuttles can be boarded to reach different destinations within the airport, such as specific terminals, subway stops, airport employee parking, etc. The second type is known as an automated people-moving system (APM) and can be configured in either personal rapid transit (PRT) or large car types. Both forms of APMs are batch systems in which passengers board vehicles that are automatically moved down either dedicated tracks or some form of right-of-way to the desired locations.

Similar to moving walkways, the impact of such systems on passenger convenience is to eliminate or reduce walking, in this case interterminal walking. Passengers electing to use automated 
people-moving systems add to their paths an element of time which includes getting to and from the access/egress points of the APM, as well as the time spent waiting for the next available car. The APM eliminates walking and may turn out to be more convenient for passengers, particularly those carrying baggage.

\section{4 - Passenger Travel Times}

The introduction of mechanical people-moving devices can greatly reduce the mandatory walking distances passengers face; however, there still exists an element of time associated with traveling between points within the airport, which in some cases may be greater for passengers using mechanical devices than for passengers traveling the same path on foot. Therefore, when assessing the potential performance of different configuration concepts (in the presence of mechanical devices), one should consider the overall travel time as a metric in addition to overall and mandatory walking distances. The following section illustrates models which can be used to estimate the time it takes to travel between points within an airport. This time does not include queuing and service times for procedures such as check-in and baggage claim. Such procedures are not necessarily influenced by terminal geometry, i.e. they are assumed to be constant across all configuration types.

\section{Basic Model}

Let the total travel time of a passenger transiting between two points $i$ and $j, t_{i j}$, be expressed as the sum of the times spent using each mode $m$ along the path from $i$ to $j, P_{i j}$, or

$$
t_{i j}=\sum_{m} t_{m} \quad \forall m \in P_{i j}
$$


Estimates for the values of $t_{m}$ depend on the particular mode used. In general, for passengers traveling by mode $m$ at a constant velocity, $v_{m}$, the estimate for $t_{m}$, is simply

$$
t_{\mathrm{m}}=\frac{d}{v_{\mathrm{m}}}
$$

where

$$
\begin{array}{lll}
t_{m} & = & \text { travel time by passengers using mode } m \\
d & = & \text { distance of segment } \\
v_{m} & = & \text { average speed of passenger using mode } m
\end{array}
$$

\section{Moving Sidewalks}

Passengers using moving sidewalks have the choice of either standing or walking along the conveyor length, denoted by $d_{m s}$. If they stand (by choice or due to congestion), their speed is simply the speed of the conveyor, $v_{\mathrm{ms}}$. If they elect to walk, their speed relative to the ground is $v_{\text {foot }}+v_{\mathrm{ms}}$, where the value of $v_{\text {foot }}$ may be significantly slower on the conveyor than in other parts of the airport due to congestion. Note that the distance the passenger actually walks is

$$
d_{\text {foot }}=t_{m s} \cdot v_{\text {foot }}
$$

and should be included in the distance matrices used to estimate overall walking distances.

\section{Automated People Movers and Shuttle Buses}

For segments covered by automated people movers and shuttle buses, the simplest model to estimate overall travel time has at least two distinct components; namely, a waiting time component and a 
transit time component. More complicated models may also consider separate boarding and disembarking times, but these more generally can be incorporated into the transit time for simplicity.

The waiting time component largely depends on the frequency of service and arrival distribution of the shuttle bus or APM. For automated people movers with a fixed headway of $h$ minutes, the average waiting time for a randomly arriving passenger at the APM depot is $h / 2$ minutes, assuming the passenger is able to board the first APM that arrives. Fixed headways are more easily maintained for APM systems because they often travel on a dedicated track and thus are not affected by traffic or congestion. Interterminal shuttle buses, on the other hand, are often exposed to traffic because they share roadways with all other vehicular traffic. Under such conditions, a fixed headway assumption is unlikely to model the arrival rate of shuttle buses adequately.

The transit time component for both shuttle buses and APMs can be modeled using the simple quotient of distance and velocity (Equation 5.17), where the velocity used represents the average speed of the vehicle over the entire path, including acceleration, deceleration, loading and disembarking times (if applicable). Note that unlike overall walking distances, travel times over a single path are stochastic if distribution assumptions are made when modeling walking speeds (which varies from passenger to passenger) and waiting time segments contained within a particular path (e.g. exponential headways for shuttle bus arrivals). Under such assumptions the travel time estimate for a single path becomes a random variable itself, with its own characteristic mean and variance.

\section{5 - Numerical Example}

As a demonstration of the techniques presented in this chapter, the following section details calculations used to obtain estimates for the expected passenger walking distances within the sample airport illustrated in Figure 5.1. We demonstrate the flexibility of the methodology by demonstrating how transition probabilities change given different levels of information regarding the distribution of passengers due to operational activities within the airport. Specifically, we show how those operational activities described in Section 5.3 might alter "base case" probabilities calculated when no information is 
Table 5.2: Preliminary Data for Sample Airport Given "Base Case" (No Information)

\begin{tabular}{ccc}
\hline Passenger & Absolute Walking \\
Category & Distance Matrix & "Uniform" \\
& & $\begin{array}{c}\text { Conditional Transition } \\
\text { Probability Matrix }\end{array}$ \\
\hline
\end{tabular}

OriginatingTerminating

$\left[\begin{array}{lllllll}50 & 100 & 50 & 265 & 265 & 265 & 265\end{array}\right]$

$\left[\begin{array}{lllllll}\frac{1}{7} & \frac{1}{7} & \frac{1}{7} & \frac{1}{7} & \frac{1}{7} & \frac{1}{7} & \frac{1}{7}\end{array}\right]$

Direct
Transfers $\left[\begin{array}{ccccccc}0 & 70 & 20 & 505 & 505 & 505 & 505 \\ 70 & 0 & 70 & 555 & 555 & 555 & 555 \\ 20 & 70 & 0 & 505 & 505 & 505 & 505 \\ 505 & 555 & 505 & 0 & 60 & 60 & 60 \\ 505 & 555 & 505 & 60 & 0 & 60 & 60 \\ 505 & 555 & 505 & 60 & 60 & 0 & 60 \\ 505 & 555 & 505 & 60 & 60 & 60 & 0\end{array}\right]$

$\left[\begin{array}{lllllll}\frac{1}{7} & \frac{1}{7} & \frac{1}{7} & \frac{1}{7} & \frac{1}{7} & \frac{1}{7} & \frac{1}{7} \\ \frac{1}{7} & \frac{1}{7} & \frac{1}{7} & \frac{1}{7} & \frac{1}{7} & \frac{1}{7} & \frac{1}{7} \\ \frac{1}{7} & \frac{1}{7} & \frac{1}{7} & \frac{1}{7} & \frac{1}{7} & \frac{1}{7} & \frac{1}{7} \\ \frac{1}{7} & \frac{1}{7} & \frac{1}{7} & \frac{1}{7} & \frac{1}{7} & \frac{1}{7} & \frac{1}{7} \\ \frac{1}{7} & \frac{1}{7} & \frac{1}{7} & \frac{1}{7} & \frac{1}{7} & \frac{1}{7} & \frac{1}{7} \\ \frac{1}{7} & \frac{1}{7} & \frac{1}{7} & \frac{1}{7} & \frac{1}{7} & \frac{1}{7} & \frac{1}{7} \\ \frac{1}{7} & \frac{1}{7} & \frac{1}{7} & \frac{1}{7} & \frac{1}{7} & \frac{1}{7} & \frac{1}{7}\end{array}\right]$

Indirect
Transfers $\left[\begin{array}{ccccccc}0 & 70 & 20 & 505 & 505 & 505 & 505 \\ 70 & 0 & 70 & 555 & 555 & 555 & 555 \\ 20 & 70 & 0 & 505 & 505 & 505 & 505 \\ 505 & 555 & 505 & 0 & 60 & 60 & 60 \\ 505 & 555 & 505 & 60 & 0 & 60 & 60 \\ 505 & 555 & 505 & 60 & 60 & 0 & 60 \\ 505 & 555 & 505 & 60 & 60 & 60 & 0\end{array}\right]$

$\left[\begin{array}{ccccccc}\frac{1}{7} & \frac{1}{7} & \frac{1}{7} & \frac{1}{7} & \frac{1}{7} & \frac{1}{7} & \frac{1}{7} \\ \frac{1}{7} & \frac{1}{7} & \frac{1}{7} & \frac{1}{7} & \frac{1}{7} & \frac{1}{7} & \frac{1}{7} \\ \frac{1}{7} & \frac{1}{7} & \frac{1}{7} & \frac{1}{7} & \frac{1}{7} & \frac{1}{7} & \frac{1}{7} \\ \frac{1}{7} & \frac{1}{7} & \frac{1}{7} & \frac{1}{7} & \frac{1}{7} & \frac{1}{7} & \frac{1}{7} \\ \frac{1}{7} & \frac{1}{7} & \frac{1}{7} & \frac{1}{7} & \frac{1}{7} & \frac{1}{7} & \frac{1}{7} \\ \frac{1}{7} & \frac{1}{7} & \frac{1}{7} & \frac{1}{7} & \frac{1}{7} & \frac{1}{7} & \frac{1}{7} \\ \frac{1}{7} & \frac{1}{7} & \frac{1}{7} & \frac{1}{7} & \frac{1}{7} & \frac{1}{7} & \frac{1}{7}\end{array}\right]$ 
initially given.

\subsection{1 - The Base Case (No Information)}

Consider again the two-terminal airport illustrated in Figure 5.1. Using the $\mathrm{x}$ - and $\mathrm{y}$-coordinates of the given locations (gates, entrances, and waypoints), it is possible to obtain values for the elements of the walking distance matrices for each of the three passenger categories described in Section 5.2. If no information is given regarding the distribution of passengers witihin the airport, we may wish to employ a "uniform" distribution assumption to determine the conditional transition probabilities (necessary to obtain walking distance estimates for each passenger category). Table 5.2 summarizes the input data associated with this "base case".

From the data given in Table 5.2, it can be shown that the conditional estimates for the expected walking distances associated with each passenger category are those listed in the first column of Table 5.4. If we further assume that the demand share matrix (distribution of aircraft among gates) is uniform, we can obtain overall expected estimates for each individual passenger category. Finally, to get an overall estimate across passenger types, we need to weight the individual estimates by the passenger mix. If we assume a passenger mix object represented by Figure 5.4(a), the overall walking distance estimate is 235.8 meters.

\subsection{2 - Intelligent Scheduling}

Now assume that in addition to the base case information, we are provided with an interterminal transition matrix, $X$, which can be used for both direct and indirect transfer passengers connecting between Terminals 1 and 2 . The values of $x_{i j}$ are

$$
X=\left[\begin{array}{ll}
.70 & .30 \\
.20 & .80
\end{array}\right]
$$


Passenger Mix Objects

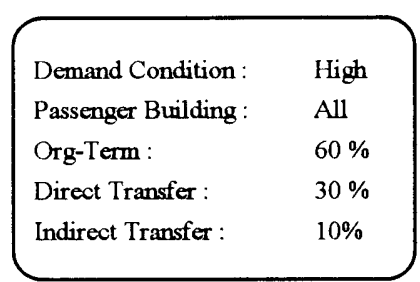

(a) High Demand

\begin{tabular}{|ll|}
\hline Demand Condition : & Low \\
Passenger Building : & 1 \\
Org-Term : & $85 \%$ \\
Direct Transfer : & $10 \%$ \\
Indirect Transfer : & $5 \%$ \\
\hline
\end{tabular}

(b) Low Demand

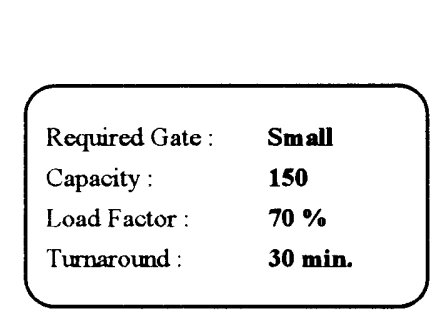

(c) Small Jets

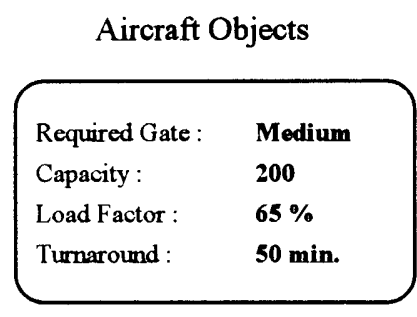

(d) Medium Jets

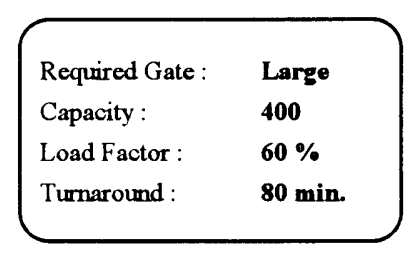

(e) Large Jets

Gate Objects

\begin{tabular}{|ll|}
\hline Capacity : & Medium \\
Use : & High Demand \\
A/C Mix : & 0\% Large \\
& $\mathbf{7 5} \%$ Medium \\
& $\mathbf{2 5} \%$ Small \\
A/C Separation : & $\mathbf{1 0 ~} \mathbf{m i n}$. \\
\hline
\end{tabular}

(f) Medium Gates $(1,2,3)$

\begin{tabular}{|ll|}
\hline Capacity : & Large \\
Use : & All Periods \\
A/C Mix : & $\mathbf{4 0} \%$ Large \\
& $\mathbf{5 0} \%$ Medium \\
& $\mathbf{1 0} \%$ Small \\
A/C Separation : & $\mathbf{1 0}$ min. \\
\hline
\end{tabular}

(g) Large Gates $(4,5,6,7)$

Figure 5.4: Possible Set of Objects 
Also let us assume that within a given departure concourse, intraterminal transitions can be approximated by a triangular distribution (for direct transfer passengers). We can thus employ Equation 5.13 to calculate individual gate-to-gate probabilities. If we assume that the probability of a direct transfer passenger departing from his or her arrival gate is .50 , it can be shown that the new transition probabilities are those listed in the first column of Table 5.3, and the overall estimates resulting from this case are those listed in the second column of Table 5.4. Note that the overall walking distance estimate has been reduced to 192.2 meters.

\subsection{3 - Aircraft Effects}

The transition probabilities calculated in the preceding cases both assume that the distribution of aircraft among gates (demand share) is uniform. If, however, we given additional information regarding the utilization of different aircraft at different gates, we can estimate a demand share matrix, $B$, as given by Equations 5.14 and 5.15. Consider the aircraft and gate objects illustrated in Figure 5.4(c-g). These objects give sufficient information to calculate the estimated number of seats, $S_{i}$, witnessed by a particular gate over a given time period (Equation 5.14), as well as the demand share, $b_{i}$, for each individual gate. The resulting demand share matrix is shown as the transition probability matrix for originating and terminating passengers in the second column of Table 5.3. Using this matrix along with the information

given for the "intelligent scheduling" case, we can obtain new estimates for the transition probabilities for the other two passenger categories. These new estimates are summarized in Table 5.3. The resulting expected walking distance estimates are summarized in the third column of Table 5.4.

\subsection{4 - Dynamic Gate Selection}

Finally, let us assume that during periods of low demand, the three medium gates located in Terminal 1 are sufficient to handle the arrival and departure operations at the airport (thus no large aircraft arrive or depart during low demand conditions). Thus, our "low-demand" configuration is simply 
a single terminal, finger-pier airport. Further, assume that during low periods the airport is used primarily by local (originating and terminating) traffic, and thus the passenger mix is better represented by the object in Figure 5.4(b). Since all airlines will be using the gates in Terminal 1, we assume that intelligent scheduling effects are negligible and aircraft effects are eliminated because all gates have the same capacity. Under these assumptions, the expected walking distance estimates can be shown to be those listed in the fourth column of Table 5.4. Note the reduction in the overall expected walking distance from the previous cases.

\subsection{5 - Summary (The Combined Case)}

If we assume that the airport operates under low demand conditions 40 percent of the time, we can incorporate the individual estimates for high and low conditions into a single "combined" estimate which represents the overall expected walking distance for the airport. Such calculations yield the estimates listed in the fifth and final column of Table 5.4. Note the influence of the dynamic gate selection on reducing the overall estimates (the third versus the fifth columns). 
Table 5.3 : Transition Probability Matrices Given Additional Information

\begin{tabular}{ccc}
$\begin{array}{c}\text { Passenger } \\
\text { Category }\end{array}$ & $\begin{array}{c}\text { Transition Probabilities } \\
\text { Under "Intelligent Scheduling" }\end{array}$ & $\begin{array}{c}\text { Transition Probabilities } \\
\text { Considering "Aircraft Effects" }\end{array}$ \\
\hline
\end{tabular}

Originating-

Terminating $\quad\left[\begin{array}{lllllll}.143 & .143 & .143 & .143 & .143 & .143 & .143\end{array}\right]\left[\begin{array}{lllllll}.133 & .133 & .133 & .151 & .151 & .151 & .151\end{array}\right]$

\begin{tabular}{|c|c|c|c|c|c|c|c|c|c|c|c|c|c|c|}
\hline \multirow{7}{*}{$\begin{array}{l}\text { Direct } \\
\text { Transfers }\end{array}$} & .350 & .077 & 273 & .075. & . 075. & . 075. & $.075]$ & {$[.336$} & .074 & .262 & .082 & .082 & .082 & .082 \\
\hline & .175 & .350 & .175 & .075 & .075 & .075 & .075 & .168 & .336 & .168 & .082 & .082 & .082 & .082 \\
\hline & 273 & .077 & .350 & . 075. & . 075. & 年 & .075 & .262 & .074 & .336 & .082 & .082 & .082 & .082 \\
\hline & .067 & .067 & .067 & .400 & .133 & .133 & .133 & .060 & .060 & .060 & .410 & 137 & .137 & .137 \\
\hline & .067 & .067 & .067 & 133 & .400 & 133 & 133 & .060 & .060 & .060 & 137 & .410 & .137 & .137 \\
\hline & .067 & .067 & .067 & 133 & 133 & .400 & 133 & .060 & .060 & .060 & .137 & 137 & .410 & 137 \\
\hline & .067 & .067 & .067 & 133 & 133 & 133 & .400 & .060 & .060 & .060 & .137 & .137 & 137. & .410 \\
\hline
\end{tabular}

Indirect
Transfers $\quad\left[\begin{array}{lllllll}.233 & .233 & .233 & .075 & .075 & .075 & .075 \\ .233 & .233 & .233 & .075 & .075 & .075 & .075 \\ .233 & .233 & .233 & .075 & .075 & .075 & .075 \\ .067 & .067 & .067 & .067 & .200 & .200 & .200 \\ .067 & .067 & .067 & .067 & .200 & .200 & .200 \\ .067 & .067 & .067 & .067 & .200 & .200 & .200 \\ .067 & .067 & .067 & .067 & .200 & .200 & .200\end{array}\right]\left[\begin{array}{lllllll}.224 & .224 & .224 & .082 & .082 & .082 & .082 \\ .224 & .224 & .224 & .082 & .082 & .082 & .082 \\ .224 & .224 & .224 & .082 & .082 & .082 & .082 \\ .060 & .060 & .060 & .205 & .205 & .205 & .205 \\ .060 & .060 & .060 & .205 & .205 & .205 & .205 \\ .060 & .060 & .060 & .205 & .205 & .205 & .205 \\ .060 & .060 & .060 & .205 & .205 & .205 & .205\end{array}\right]$ 
Table 5.4: Summary of Estimates for Expected Walking Distances

\begin{tabular}{|c|c|c|c|c|c|}
\hline Category & $\begin{array}{l}\text { Estimates } \\
\text { Under } \\
\text { "Base Case" }\end{array}$ & $\begin{array}{l}\text { Estimates } \\
\text { Under } \\
\text { "Intelligent } \\
\text { Scheduling" }\end{array}$ & $\begin{array}{l}\text { Estimates } \\
\text { Including } \\
\text { "Aircraft } \\
\text { Effects" }\end{array}$ & $\begin{array}{c}\text { Estimates } \\
\text { for } \\
\text { Low Demand } \\
\text { Periods }\end{array}$ & $\begin{array}{l}\text { Combined } \\
\text { "All Demand } \\
\text { Periods" } \\
\text { Case }\end{array}$ \\
\hline $\begin{array}{l}\text { Originating- } \\
\text { Terminating }\end{array}$ & {$[180.0]$} & {$[180.0]$} & {$[186.1]$} & {$[66.7]$} & {$[138.3]$} \\
\hline $\begin{array}{c}\text { Direct } \\
\text { Transfers }\end{array}$ & {$\left[\begin{array}{l}301.4 \\
337.1 \\
301.4 \\
249.3 \\
249.3 \\
249.3 \\
249.3\end{array}\right]$} & {$\left[\begin{array}{l}162.4 \\
191.0 \\
162.4 \\
128.3 \\
128.3 \\
128.3 \\
128.3\end{array}\right]$} & {$\left[\begin{array}{l}175.7 \\
205.2 \\
175.7 \\
118.7 \\
118.7 \\
118.7 \\
118.7\end{array}\right]$} & {$\left[\begin{array}{c}22.5 \\
35.0 \\
22.5 \\
0 \\
0 \\
0 \\
0\end{array}\right]$} & {$\left[\begin{array}{c}114.4 \\
137.1 \\
114.4 \\
71.2 \\
71.2 \\
71.2 \\
71.2\end{array}\right]$} \\
\hline $\begin{array}{l}\text { Indirect } \\
\text { Transfers }\end{array}$ & {$\left[\begin{array}{l}334.3 \\
384.3 \\
334.3 \\
520.7 \\
520.7 \\
520.7 \\
520.7\end{array}\right]$} & {$\left[\begin{array}{l}226.2 \\
276.2 \\
226.2 \\
520.3 \\
520.3 \\
520.3 \\
520.3\end{array}\right]$} & {$\left[\begin{array}{l}237.0 \\
287.0 \\
237.0 \\
520.3 \\
520.3 \\
520.3 \\
520.3\end{array}\right]$} & {$\left[\begin{array}{c}106.7 \\
156.7 \\
106.7 \\
0 \\
0 \\
0 \\
0\end{array}\right]$} & {$\left[\begin{array}{l}184.9 \\
234.9 \\
184.9 \\
312.2 \\
312.2 \\
312.2 \\
312.2\end{array}\right]$} \\
\hline $\begin{array}{l}\text { Estimates } \\
\text { by } \\
\text { Category }\end{array}$ & {$\left[\begin{array}{l}180.0 \\
276.7 \\
448.0\end{array}\right]$} & {$\left[\begin{array}{l}180.0 \\
147.0 \\
401.4\end{array}\right]$} & {$\left[\begin{array}{l}186.1 \\
145.3 \\
414.2\end{array}\right]$} & {$\left[\begin{array}{c}66.7 \\
26.7 \\
123.3\end{array}\right]$} & {$\left[\begin{array}{c}138.3 \\
97.9 \\
297.8\end{array}\right]$} \\
\hline $\begin{array}{c}\text { Overall } \\
\text { Estimate }\end{array}$ & {$[235.8]^{1}$} & {$[192.2]^{1}$} & {$[196.7]^{1}$} & {$[65.5]^{2}$} & {$[144.2]$.} \\
\hline
\end{tabular}

${ }^{1}$ Note : Assumes a passenger mix of [.60 .30 .10$]$

${ }^{2}$ Note : Assumes a passenger mix of [.85 .10 .05] 


\title{
Chapter 6
}

\section{Some Numerical Results}

\author{
Introduction
}

Chapter $\mathbf{4}$ demonstrated that it is possible to characterize geometrically several different configurations of airport passenger buildings, and from these characterizations it is possible to determine. the absolute distances between any two points within them. Chapter 5 demonstrated how we can estimate the probability that each of these distances will be traversed based on assumptions regarding the distribution of passengers. Given matrices for the absolute distances traveled by each of the different passenger categories and corresponding matrices for the unconditional transition probabilities, it is possible to estimate various measures of potential configuration performance based on well-known results of geometrical probability. Examples of these measures in the case of walking distances include :

\footnotetext{
- the mean or overall expected distance

- the variance or standard deviation

- $\quad$ the mean excess distance above some threshold

- the probability of excess walking
}

Similar measures can also be determined for mandatory walking distances and overall travel times, depending on the amount of information given to the planner. 
Chapter 6 illustrates the method of estimating the potential performance of a configuration by applying the models presented in Chapter 5 to a number of standard passenger building configurations. The intent of this chapter is to demonstrate the usefulness of the technique, however, and not to provide an exhaustive examination of each configuration in order to determine the "best" overall for any set of conditions (it is unlikely that such a configuration exists). Section 6.1 describes the general experimental method used to demonstrate our models and introduces a graphical tool called the "performance profile". Section 6.2 compares as a proof of concept some preliminary numerical results of our model with those obtained from other authors. Section 6.3 then details specific numerical data used in a more general exploration of a variety of different configuration concepts. Section 6.4 demonstrates how performance profiles can be used to examine the robustness of a given configuration to different levels and types of transfer traffic. Section 6.5 demonstrates how these profiles can be used to examine robustness to potential changes in industry structure. Finally, Section 6.6 demonstrates how performance profiles can be used to explore the robustness of configurations to increases in size (as measured by the total number of gates).

\section{1 - Experimental Method}

Our method of selecting an initial passenger building configuration (based on specified measures of airport performance) is a two step process. Step One begins with the selection of the most appropriate geometry for each of the configuration concepts considered (i.e. the number and size of the individual concourses). Once the most appropriate geometry for each concept has been selected, Step Two then compares performance across concepts to determine which would be the most robust to various performance criteria.

Note that the order in which comparisons are made (within a particular concept and then among different concepts) is actually the reverse of the order implied by the two step process of preparing a terminal layout plan as specified by the FAA [1971]. Typically, the selection process is thought of as going from the general to the more specific, i.e. first select the overall shape of the building configuration 
(finger-pier, circular satellite, midfield " $\mathrm{X}$ "), and then select the number and size of the individual concourses.

Such a selection procedure, however, tacitly assumes that the performance of a given configuration is independent of its particular geometry. In other words, it neglects the possibility that a specific geometry for one concept might outperform a different geometry from another, and vice versa. For example, one might (perhaps incorrectly) choose circular shaped-satellites over rectangular-shaped satellites (i.e. number of gates) because for a particular size, the circular satellites may have a shorter maximum distance between any two gates. A geometry with a greater number of rectangular satellites (and thus fewer gates per satellite), however, may outperform the circular satellite concept for all possible geometries considered.

Thus it is important first to explore the behavior of the different possible geometries (for a particular configuration), and then select the most appropriate concept from among the best performing geometries in order to determine which is the most appropriate for the given conditions. We accomplish this selection through a graphical representation of the data called a "performance profile". A description of performance profiles follows in the next section.

\section{Performance Profiles}

For a specific configuration (i.e. gate arrival, finger-pier, satellite) with a given number of gates, many different geometries are possible. For example, a 24-gate airport with equally sized concourses can be configured as having two concourses each with 12-gates, three with 8-gates, etc. For a given set of conditions, a plot can be made to demonstrate how each geometry would potentially perform. Additional plots can be made for different sets of conditions to show how each geometry might perform over a range of circumstances. We denote the aggregation of these plots onto a single set of axes a performance profile.

One example of a performance profile for a centralized, circular satellite configuration (Figure 4.5) is shown in Figure 6.1. Each line represents the potential performance (as measured by expected 
walking distance) of the different geometries for varying levels of transfer traffic, $P$ (the fraction of total traffic made up of transfer passengers). From these three lines, we can conclude that in each case, the overall expected walking distance is minimized when the number of concourses, $n$, equals four. Thus, if our stated objective was to select the configuration geometry that minimized overall walking distances, the most appropriate configuration would contain four concourses with six gates each.

Moreover, we can conclude from the profile that the decision of choosing the most appropriate geometry is independent of the level of transfer traffic, $P$. Thus, by representing potential performance in terms of a profile rather than a single number, we can do more than simply find the most appropriate geometry -- we demonstrate that the margin of error related to the passenger mix forecast is irrelevant to this particular decision. As mentioned previously, the passenger mix forecast has historically been shown to be subject to a great deal of uncertainty (Dallas/Ft. Worth), and conclusions drawn based on a single mix forecast can lead to inappropriate design decisions. In a long-term planning context, the identification that the most appropriate geometry is independent of the level of transfer traffic, if such a property exists, is likely to be more important to planners than the specific numerical estimates generated for the particular profile (because the error associated with the passenger mix forecast will not affect the selection decision).

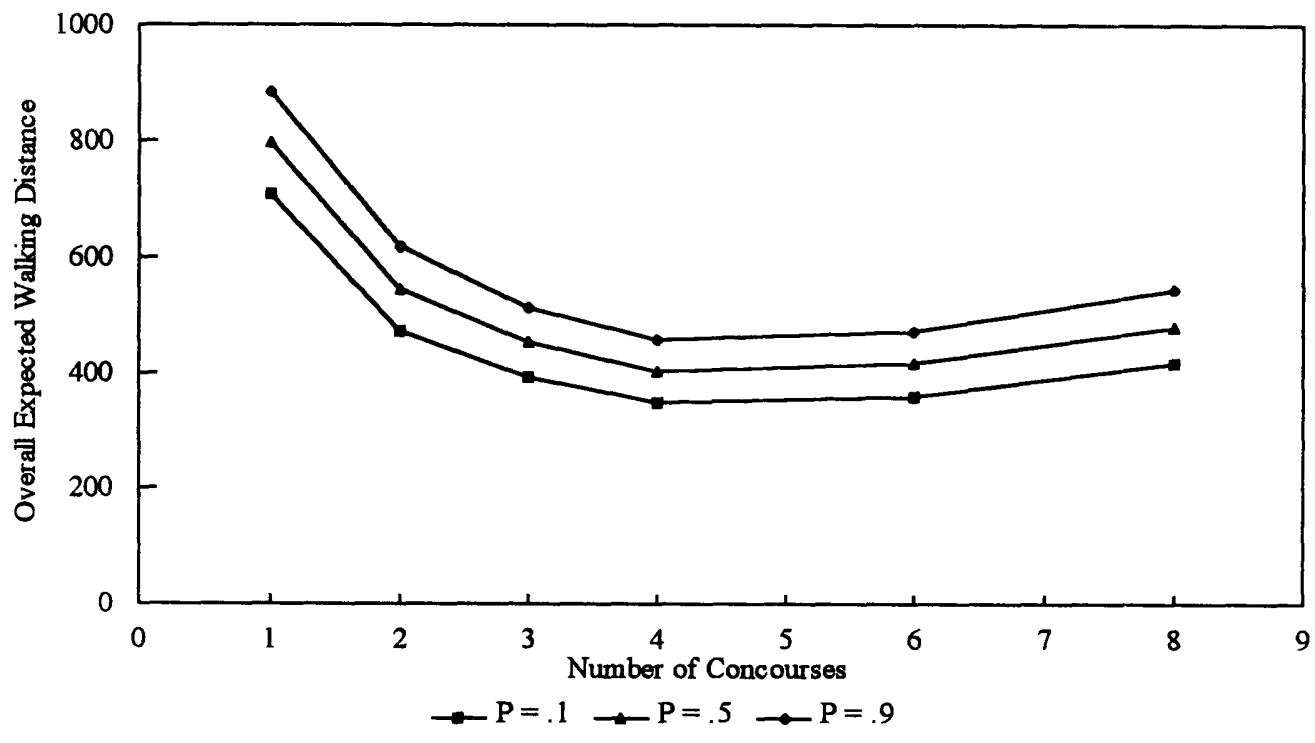

Figure 6.1 : Performance Profile for Centralized, Circular Satellite Configuration 
The following section demonstrates how the use of performance profiles can be used to replicate analyses made by other authors who have addressed the issue of selecting the most appropriate passenger building configurations for a given set of conditions.

\section{2 - Initial Proof of Concept}

This section compares the results obtained by our models with those of Wirasinghe, Bandara, and Vandebona [1987] in their paper "Airport Terminal Geometries for Minimal Walking Distances". In that paper, the authors explored the potential performance of three different finger-pier configuration concepts, which they denote

- Type 1: Centralized - Radial

- Type 2: Centralized - Standard

- $\quad$ Type 3 : Semi-Centralized

Figure 6.2 illustrates the basic geometric features of these three concepts. Tables 6.1 and 6.2 summarize the input and output data, respectively, used to obtain walking distance estimates for the different possible geometries associated with each concept.

Table 6.1 : Input Parameters Used in WBV

$S \quad$ Spacing between parallel pier base centerlines (Type 2,3)

$S_{1} \quad$ Perpendicular clearance required at finger base (Type 1)

$S_{g} \quad$ Gate spacing

$b_{2} \quad$ Expected walking distance within centralized terminal blocks

$b_{3} \quad$ Expected walking distance within semi-centralized terminal blocks

$W_{A} \quad$ Threshold for excess walking
$230 \mathrm{~m}$

$115 \mathrm{~m}$

$60 \mathrm{~m}$

$40 \mathrm{~m}$

$20 \mathrm{~m}$

$350 \mathrm{~m}$ 
Table 6.2 : Output Parameters Used in WBV
$n \quad$ Number of individual concourses
$g \quad$ Number of gates per individual concourse
$\mu_{\mathrm{w}} \quad$ Overall expected walking distance
$\sigma_{\mathrm{w}} \quad$ Standard deviation of walking distance
$\mu_{\mathrm{E}} \quad$ Mean excess walking distance above threshold
$P_{E} \quad$ Probability of excess walking

\section{Differences in Assumptions}

By constructing similar geometries (using the methods described in Chapter 4) and employing similar assumptions regarding the distribution of passengers, we should be able to reproduce the results obtained in WBV using analytic techniques. There is, however, one fundamental difference between the two techniques that prevents achieving identical results. WBV uses continuous approximations in order to construct analytic formulae to describe potential configuration performance. Such an approximation assumes that passenger arrival and departure points are uniformly distributed along the entire length of the pier rather than at discrete points (i.e. gates). This assumption introduces an error into the walking distance calculation that is inversely proportional to the number of gates within a concourse (e.g. in a concourse with four gates $(g=4)$, the potential error associated with a continuous approximation is 25 percent) [Wirasinghe 1988].

Further, the continuous approximation allows for a non-integer allocation of gates to concourses for a given total number of gates, $G$. Thus, for $G=25$, it is possible to obtain an optimal geometry having six identically sized concourses, each containing $4 \frac{1}{6}$ gates. Our technique of enumerating discrete paths and weighting the absolute distances by the (expected) frequency each path will be walked ensures, by contrast, integer solutions. Thus, the results obtained by each method will differ somewhat in the final selection of the most appropriate geometry. 


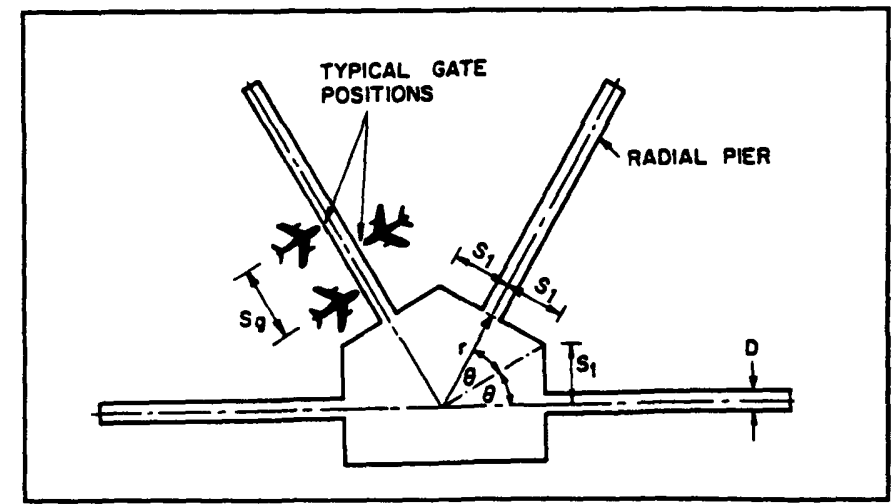

(a) Type 1 - Centralized Radial

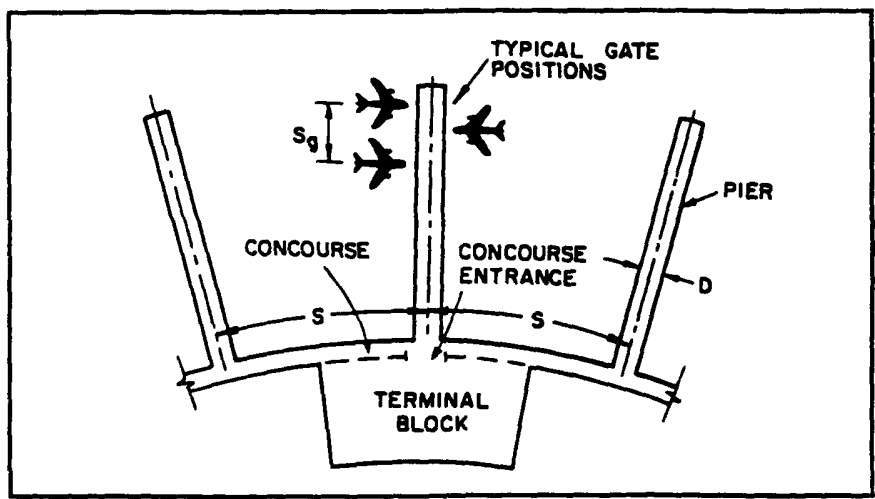

(b) Type 2 - Centralized Standard

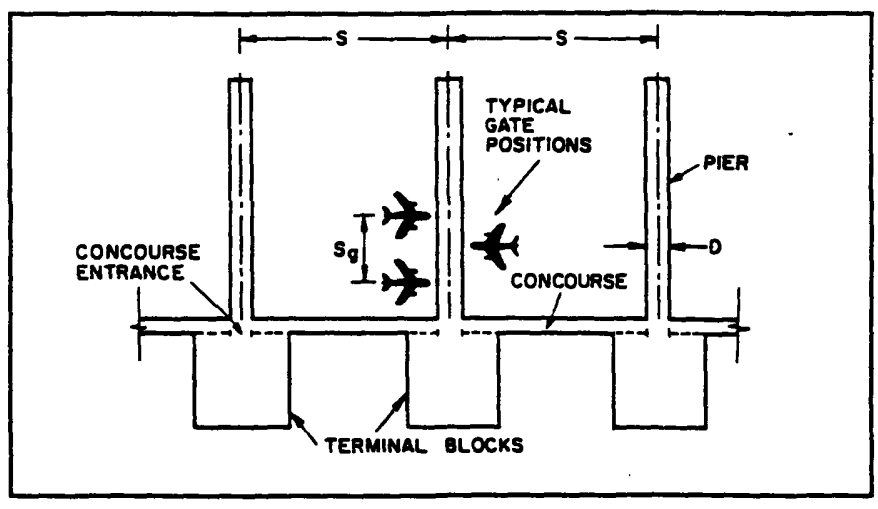

(c) Type 3 - Semi-Centralized

Figure 6.2: Geometric Constructions of Finger Pier Configurations in WBV 1

\footnotetext{
${ }^{1}$ Source : [Wirasinghe et al. 1987], p. 486,488,489.
} 


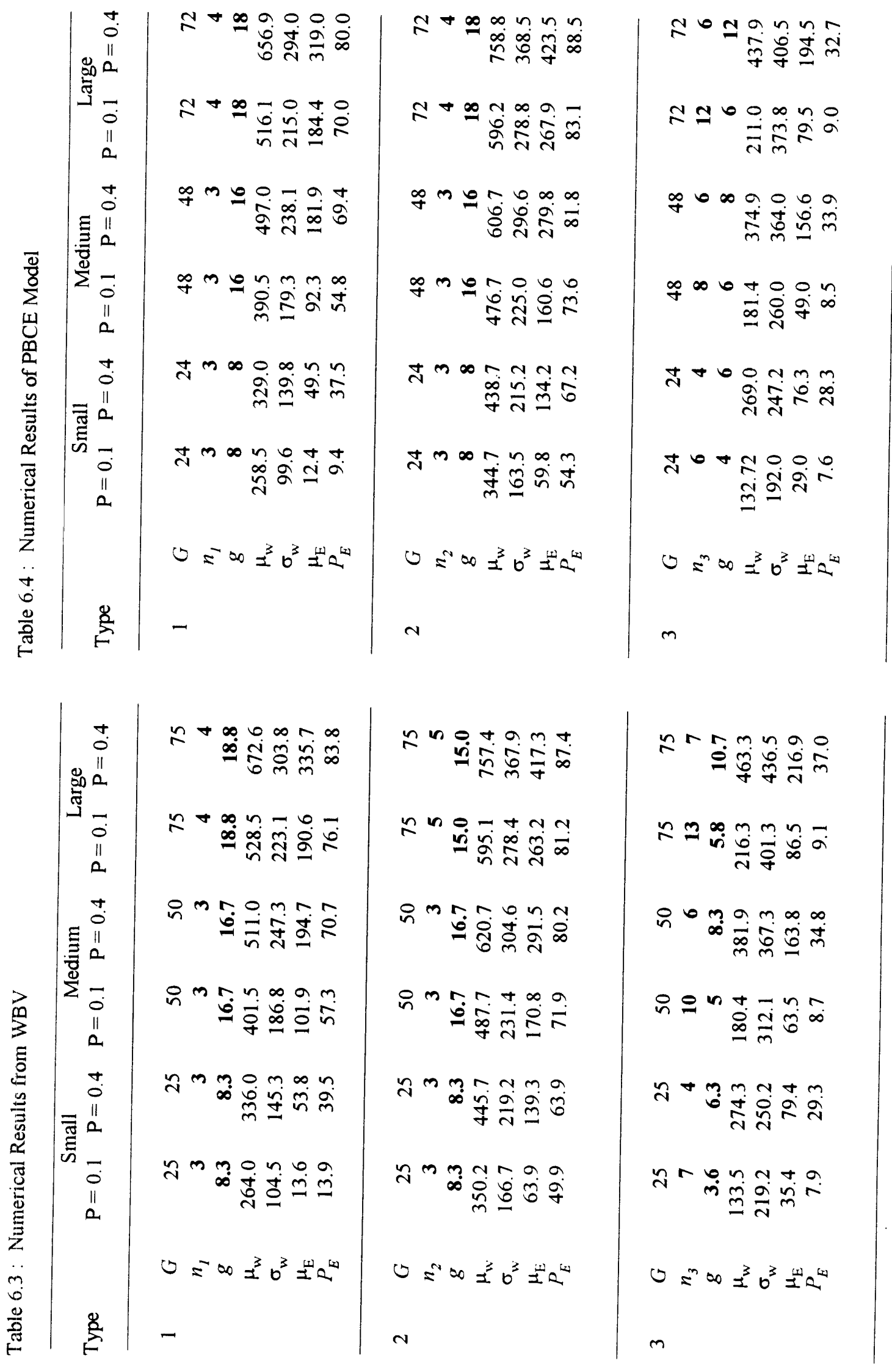




\section{Results}

Tables 6.3 and 6.4 summarize the results of our numerical test. The values for the total number of gates, $G$, used in the PBCE Model for Small, Medium, and Large airports (24, 48, and 72 respectively) were selected for their large number of integer factors (thus facilitating more possible geometries than the values for $G$ used in WBV). Therefore, the number of gates per concourse $(g)$ in Table 6.3 do not necessarily equal the number of gates per concourse in Table 6.4. Nevertheless, the two models produce reasonably consistent results for the given input data. Specifically, note the similarity between the two models in the "optimal" number of concourses, $n$, and corresponding number of gates per concourse, $g$, (considering the differing assumptions regarding the integrality of solutions).

Both models demonstrate that the decision of selecting the most appropriate geometry for the first two configuration types is relatively insensitive to values of $P$ between $P=0.1$ and $P=0.4$, unlike the Type 3 configuration. For the semi-centralized concept, higher levels of transfer traffic (i.e. a higher $P$ ) change the most appropriate configuration significantly for all values of $G$. Figure 6.3 illustrates how the use of a performance profile can enhance the numerical information available to decision makers when selecting the most appropriate geometry for the Type 3 configurations (in this case, for $G=72$ ).

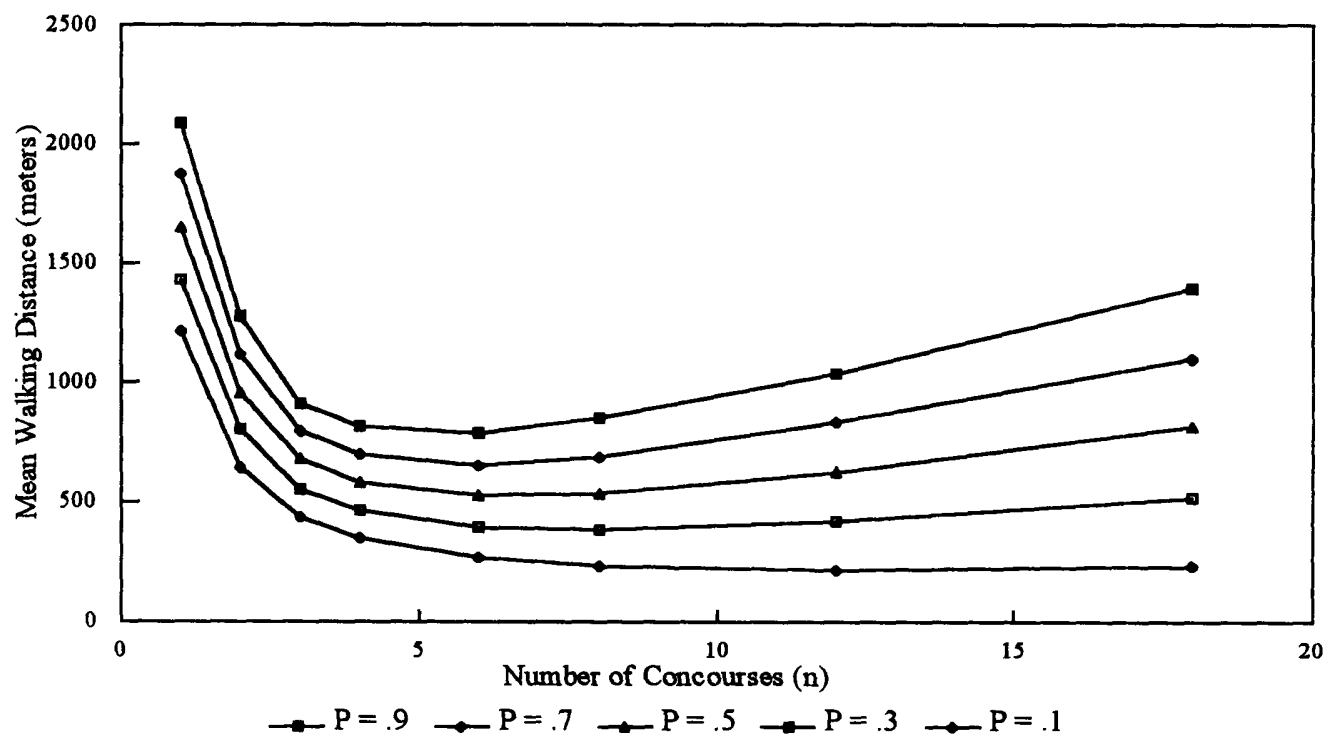

Figure 6.3 : Performance Profile for Type 3 Configuration $(G=72)$ 
Each line in the profile shows the behavior of the mean walking distance as a function of the number of individual concourses, $n$, for a different level of transfer traffic, $P$. Note that as the level of transfer traffic increases ( $P$ approaches 1.0), the behavior of the curve changes. For low levels of transfer traffic, the most appropriate geometry (to minimize walking distances) has many individual concourses with only a few gates each. For higher levels of transfer traffic, the most appropriate geometry has fewer concourses, each containing more gates. Therefore, the performance of the given configuration is highly dependent on the expected level of transfer traffic.

Figure 6.4 illustrates the estimates of potential performance for two of the geometries shown in Figure 6.3; namely $n=4$ and $n=18$. Although the $n=18$ geometry performs better for values of $P$ less than .25 , the $n=4$ geometry is more consistent over the entire possible range of values, i.e. it is more robust to changes in the level of transfer traffic.

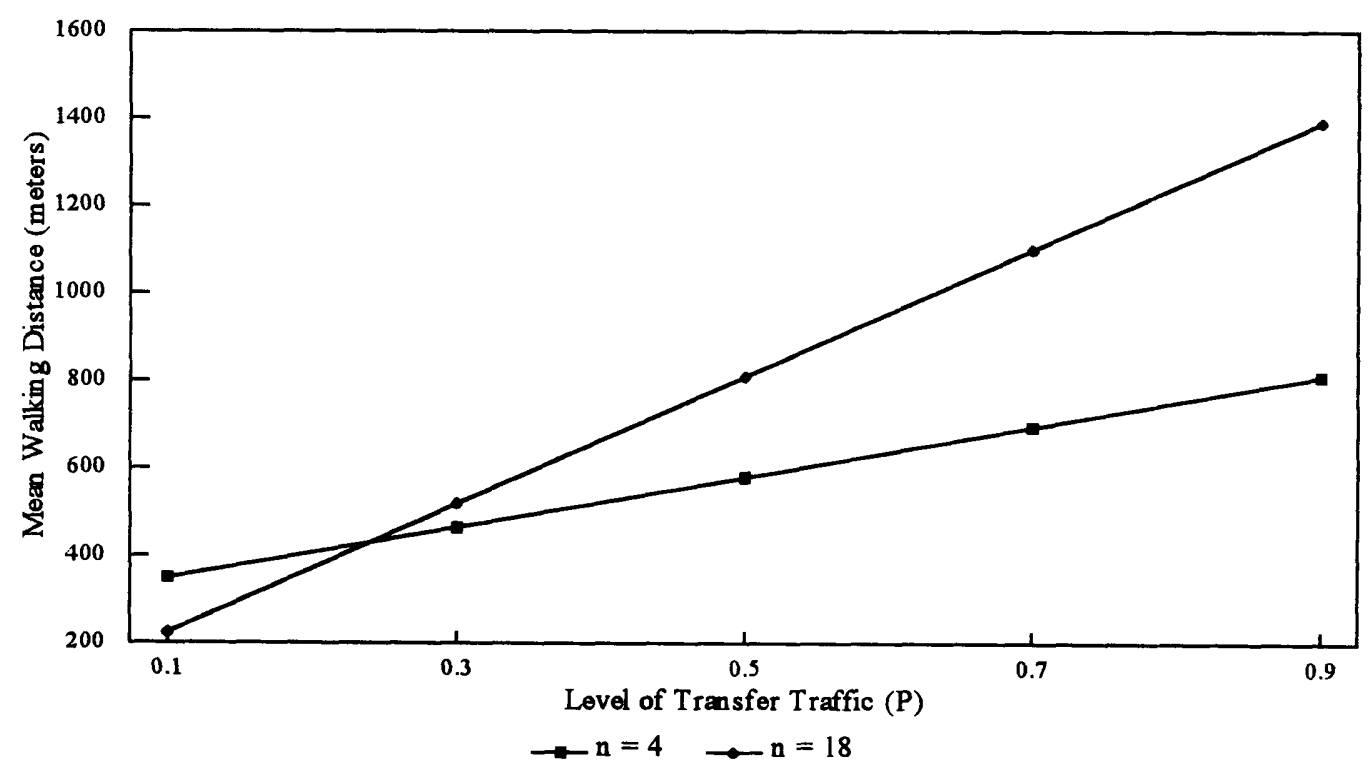

Figure 6.4 : Performance Robustness to $P$ for Two Different Geometries

The following sections show how similar analyses can be performed on different configuration types to explore the behavior of their potential performance. 


\section{3 - Experimental Setup}

This section details the specific parameter values used to generate performance profiles for several different passenger building configurations. Many of the values used are taken from previous authors to continue the proof of concept as well as to maintain consistency in the conclusions drawn from numerical results. It is important to note, however, that the specific values are secondary to the demonstration of the technique used to assess potential performance.

\subsection{1 - Configurations and Geometries Considered}

Table 6.5 summarizes the configuration concepts considered for analysis. For each of the four primary concepts described in Chapters 3 and 4 (gate arrival, pier, satellite, and midfield), centralized and decentralized versions are considered. Note, however, that we do not consider a decentralized, parallel linear midfield configuration. Based on the geometric characterizations described in Chapter 4 , decentralizing the parallel linear configuration would be equivalent to having a dedicated terminal block for each of the midfield concourses. Such a configuration is already described either by the decentralized rectangular satellite concept, or the decentralized parallel pier concept, depending on the assumptions made regarding the location of the terminal blocks relative to the individual concourses.

For our initial analyses, we set the size of the airport (as measured by the total number of gates, $G)$ to be $\mathbf{2 4}$ gates. This size was chosen to reflect potential performance of a small to moderately sized airport and because the number 24 has several whole number factors which can be used to generate different configuration geometries. We maintain the assumption that for a given geometry, all concourses are identically sized and shaped. As mentioned in Chapter 2, although previous research has suggested incremental gains can be achieved (in some configurations) using unevenly shaped concourses, such geometries neglect two important issues; namely, the possibility that conditions may change which make the uneven sizes inappropriate, and the undesirability of smaller, more remote concourses to the airlines which must operate within them. The methodology is sufficiently general, however, to consider any 
geometry, including those with unevenly sized concourses. We simply do not consider them in our analysis for the aforementioned reasons. Table 6.6 illustrates the specific geometries considered for each of the standard configurations listed in Table 6.5.

Note that for " $\mathrm{T}$ "-shaped satellite and "X"-shaped midfield configuration concepts, not all values of $n$ are considered. For "T"-shaped satellites, we consider only geometries which generate values of $g$ that are an integer multiple of three. Similarly, for "X"-shaped midfield configurations we consider only geometries which generate values of $g$ that are an integer multiple of four. Although theoretically possible, geometries for which $g$ is not an even multiple of three and four, respectively, are somewhat unrealistic because they do not fully exploit the advantage of the concourse shape.

\subsection{2 - Centralized Concepts}

Table 6.7 summarizes the parameter values used in our analyses of centralized configuration concepts. For all four configuration types (gate arrival, pier, satellite and midfield), we assume the distance walked by passengers within the terminal block, $b_{p}$, is constant at $40 \mathrm{~m}$. For parallel pier configurations, we assume the distance between adjacent concourse centerlines is $230 \mathrm{~m}$. For satellite configurations, two parameter values are needed to describe spacing requirements; namely, $S$, the distance between two adjacent satellite concourses, and, $B$, the distance between a terminal block and its corresponding satellite concourse.

The value of $S$ is set to be $190 \mathrm{~m}$, while the value of $B$ (subscripted by the appropriate concourse shape) has a lower bound of $190 \mathrm{~m}$. Due to the limits placed on the separation of satellites by the maximum angle of spread, $\beta$, the value of $B$ may be higher than the lower bound (see Equations 4.6 and 4.7). Similarly, for the midfield $X$ configuration the value of $B_{X}$ is set at a lower bound of $190 \mathrm{~m}$, and the length of the connector to each midfield concourse, $C_{X}$, is calculated using Equations 4.9 through 4.11. 
Table 6.5: Configurations Considered for Analysis

\begin{tabular}{|l|c|c|}
\hline Description & Centralized & Decentralized \\
Gate Arrival & $\bullet$ & $\bullet$ \\
Parallel Pier & $\bullet$ & $\bullet$ \\
Satellites & & $\bullet$ \\
Circular & $\bullet$ & $\bullet$ \\
Rectangular & $\bullet$ & $\bullet$ \\
"T"-Shaped & $\bullet$ & \\
Midfield & & \\
Parallel Linear & $\bullet$ & \\
Midfield "X" & $\bullet$ & \\
\hline
\end{tabular}

Table 6.6: Possible Geometries for $G=24$

\begin{tabular}{|l|c|c|c|c|c|c|}
\hline Description & $\underline{\mathrm{n}=1}$ & $\underline{\mathrm{n}=2}$ & $\underline{\mathrm{n}=3}$ & $\underline{\mathrm{n}=4}$ & $\underline{\mathrm{n}=6}$ & $\underline{\mathrm{n}=8}$ \\
Gate Arrival & $\bullet$ & $\bullet$ & $\bullet$ & $\bullet$ & $\bullet$ & $\bullet$ \\
Parallel Pier & $\bullet$ & $\bullet$ & $\bullet$ & $\bullet$ & $\bullet$ & $\bullet$ \\
Satellites & & & & & & \\
Circular & $\bullet$ & $\bullet$ & $\bullet$ & $\bullet$ & $\bullet$ & $\bullet$ \\
Rectangular & $\bullet$ & $\bullet$ & $\bullet$ & $\bullet$ & $\bullet$ & $\bullet$ \\
"T"-Shaped & $\bullet$ & $\bullet$ & & $\bullet$ & & $\bullet$ \\
Midfield & & & & & & \\
Parallel Linear & $\bullet$ & $\bullet$ & $\bullet$ & $\bullet$ & $\bullet$ & $\bullet$ \\
Midfield "X" & $\bullet$ & & $\bullet$ & & $\bullet$ & \\
\hline
\end{tabular}


Table 6.7 - Parameters Used in Centralized Configurations

\begin{tabular}{|c|c|c|}
\hline Parameter & Description & Value [Min. Max] \\
\hline $\begin{array}{l}\text { Global } \\
S_{a}\end{array}$ & Gate spacing & $60 \mathrm{~m}$ \\
\hline$b_{p}^{g}$ & Distance walked within terminal block & $40 \mathrm{~m}$ \\
\hline \multicolumn{3}{|l|}{ Pier } \\
\hline$S$ & Spacing between parallel pier concourses & $230 \mathrm{~m}$ \\
\hline \multicolumn{3}{|l|}{ Satellite } \\
\hline$S$ & Spacing between satellite concourses & $190 \mathrm{~m}$ \\
\hline$\beta$ & Maximum angle of spread for satellites & {$\left[180^{\circ}, 270^{\circ}\right]$} \\
\hline$R_{S}$ & Radius of circular satellite concourse & Derived \\
\hline$B_{C}$ & $\begin{array}{l}\text { Spacing between terminal block and circular } \\
\text { satellite concourse }\end{array}$ & [190 m, Derived] \\
\hline$L_{R}$ & Length of rectangular satellite concourse & Derived \\
\hline$B_{R}$ & $\begin{array}{l}\text { Spacing between terminal block and } \\
\text { rectangular satellite concourse }\end{array}$ & [190 m, Derived] \\
\hline$L_{T}$ & $\begin{array}{l}\text { Length of main arm of " } T \text { "-shaped satellite } \\
\text { concourse }\end{array}$ & Derived \\
\hline$B_{T}$ & $\begin{array}{l}\text { Spacing between terminal block and } \\
\text { "T"-shaped satellite concourse }\end{array}$ & [190 m, Derived] \\
\hline
\end{tabular}

\begin{tabular}{|c|c|c|}
\hline $\begin{array}{l}\text { Midfield } \\
S_{P L}\end{array}$ & Spacing between linear midfield concourses & $230 \mathrm{~m}$ \\
\hline$B_{P L}$ & $\begin{array}{l}\text { Spacing between terminal block and first } \\
\text { parallel linear midfield concourse }\end{array}$ & $190 \mathrm{~m}$ \\
\hline$S$ & Spacing between midfield " $\mathrm{X}$ " concourses & $190 \mathrm{~m}$ \\
\hline$L_{X}$ & Length of one arm of "X"-shaped concourse & Derived \\
\hline$B_{X}$ & $\begin{array}{l}\text { Spacing between terminal block and "X"- } \\
\text { shaped midfield concourse }\end{array}$ & $190 \mathrm{~m}$ \\
\hline$C_{X}$ & $\begin{array}{l}\text { Length of connector to midfield concourse } \\
\text { imposed by spacing } B_{x}\end{array}$ & Derived \\
\hline
\end{tabular}




\subsection{3 - Decentralized Concepts}

Table 6.8 summarizes the parameter values used in our analyses of decentralized configuration concepts. We now assume that for all four concept types, the distance $b_{p}$ walked within each dedicated terminal block is half the distance it was in the centralized terminal block, or $20 \mathrm{~m}$. We again assume that the distance between adjacent, parallel concourse centerlines, is $230 \mathrm{~m}$.

As described in Chapter 4, one way to characterize decentralized satellite configurations is to hold constant the distance, $B$, between a terminal block and its corresponding satellite. The mandatory spacing between adjacent satellites, $S$, can be then maintained by varying the distance between adjacent terminal blocks, $S_{C}, S_{R}$, or $S_{T}$. For our analyses, the value of $B$ is set to be $130 \mathrm{~m}$, the value of $S$ is set to be $190 \mathrm{~m}$. The values of $S_{C}, S_{R}$, and $S_{T}$ are determined by Equations 4.12 through 4.16. Midfield $X$ configurations are also characterized by a constant $B_{X}$ of $130 \mathrm{~m}$, a constant $S$ of $190 \mathrm{~m}$, and a separation of terminal blocks, $S_{X}$, determined by Equations 4.17 through 4.21 .

\subsection{4 - Performance Robustness}

Using the experimental setup described in the previous sections, it is possible to estimate measures of potential performance as described in Chapter 5. By estimating these measures for each possible geometry listed in Table 6.6, profile lines can be generated to determine the most appropriate geometry for a given condition. By generating profile lines for a range of conditions, the most robust geometry (to the particular parameter being varied) can be selected. By employing such an approach, we can explore configuration robustness to a variety of factors, including: the level and type (direct or indirect) of transfer traffic, potential changes in the industry structure, and size (as measured by the number of total gates). The following sections describe possible ways to characterize these factors in order to generate such profiles. 
Table 6.8 - Parameters Used in Decentralized Terminal Configurations

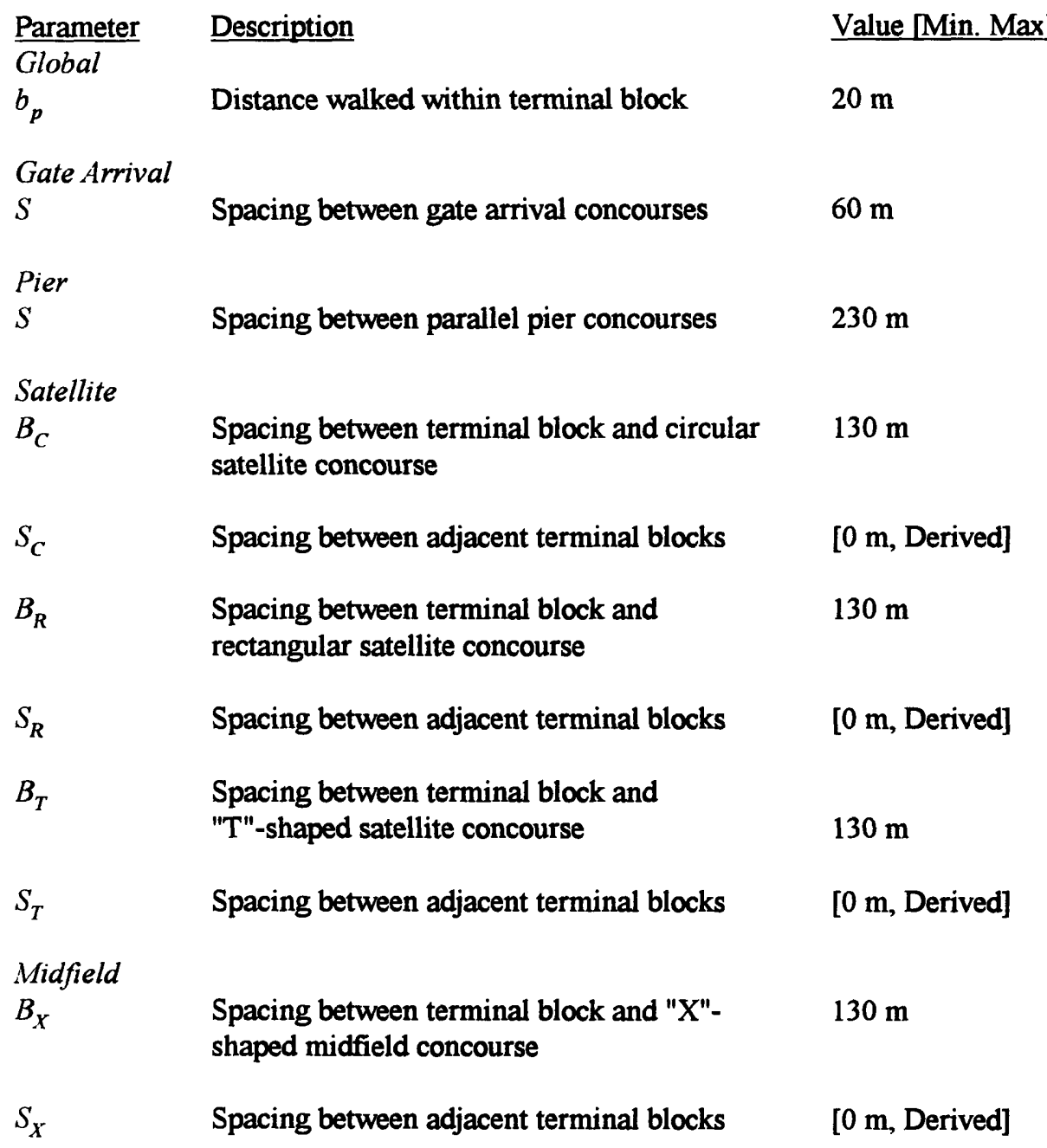




\section{Robustness to Level and Type of Transfer Traffic}

To test the robustness of different configurations to the level of transfer traffic, we can simply vary the value of the transfer traffic attributes contained within the passenger mix object, as illustrated in Table 5.1. Bandara [1989] defines the parameter $P$, to represent the proportion of total traffic that is made up of connecting passengers. The range of values for $P$ is $[0,1]$, where at $P=0$, the traffic mix consists entirely of originating and terminating passengers, and at a value of $P=1$, the mix consists entirely of transfers.

To test performance robustness to the level of hub operations, Bandara [1989] defines an additional parameter, $Q$, to represent the proportion of transfer traffic that is able to make direct (or hub) connections, those not requiring any intermediate services (e.g. intraline transfers). For an ordered pair $(P, Q)$ of $(.4, .7)$, for example, the total proportion of transfer traffic is 40 percent (60 percent originating and terminating traffic), of which 70 percent are able to make direct transfers (28 percent of the total traffic population). The remaining 30 percent (12 percent of the total traffic population) are indirect (or non-hub) transfers who require intermediate services. In the object-oriented representation of the airport environment, any ordered pair $(P, Q)$ is represented by the following passenger mix object (Figure 6.5) :

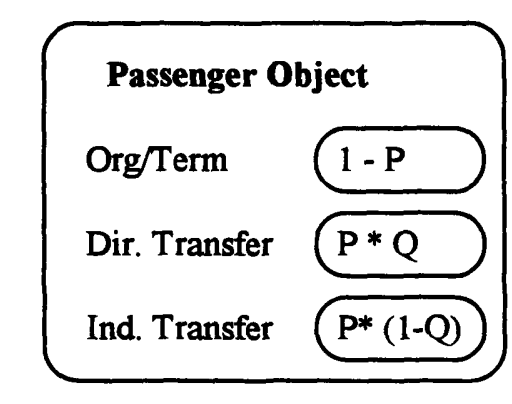

Figure 6.5 : Passenger Mix Object

The two dimensional sample space bounded by the possible values of $(P, Q)$ constitute the domain of possible traffic mixes an airport may witness. Thus, by testing configuration robustness to a 
representative sample of points within the $(P, Q)$ sample space, it is possible to assess which configurations (and which geometries of those configurations) would perform most appropriately over changes in the level and type of transfer traffic. Some representative results of such experiments are provided in Section 6.4 .

\section{Robustness to Industry Structure}

To test the robustness of different configurations to the effects of industry structure (as measured by carrier presence at an airport), we can use the parameter $r$, which Bandara [1989] defines as the fraction of direct transfer traffic that is certain to depart from their arrival concourse. In some sense, higher values of $r$ can be associated with a stronger carrier presence, as it could reflect the use of effective stand management policies employed by the carrier in trying to maintain high connection reliability. The parameter is not perfect, however, and high values of $r$ are not necessarily tantamount to carrier dominance.

For example, United Airlines operates two similarly sized concourses in Terminal 1 at Chicago / O'Hare, where it is the dominant carrier (as measured by market share). As a first approximation, we might assume that a direct transfer passenger is equally likely to depart from either concourse $(r=.5)$. America West Airlines, on the other hand, operates only a handful of gates at O'Hare, and thus their intraline transfer passengers are almost certain to depart from their arrival concourse $(r \cong 1.0)$. Thus it is important when using measures such as $r$ to consider what the particular values mean in the context of the airport being considered.

In an object-oriented representation of the airport environment, the parameter $r$ can be considered explicitly in the matrix of interterminal transitions, $X$, described in Chapter 5 . For an airport with $n$ concourses, the interterminal transition matrix, $X$, which incorporates the definition of $r$ described 
by Bandara [1989] is :

$$
X=\left[\begin{array}{ccccc}
r+\frac{1-r}{n} & \frac{1-r}{n} & \frac{1-r}{n} & \frac{1-r}{n} & \frac{1-r}{n} \\
\frac{1-r}{n} & \cdots & & & \frac{1-r}{n} \\
\frac{1-r}{n} & & r+\frac{1-r}{n} & & \frac{1-r}{n} \\
\frac{1-r}{n} & & & \cdots & \frac{1-r}{n} \\
\frac{1-r}{n} & \frac{1-r}{n} & \frac{1-r}{n} & \frac{1-r}{n} & r+\frac{1-r}{n}
\end{array}\right]
$$

The possible sample space for testing performance robustness can now be described in three dimensions, bounded by the possible values of $P, Q$, and $r$. Each ordered triplet now represents a specific level of transfer traffic, the degree of hub operations in place (as measured by the transfer split), and a measure of the industry structure. Performance profiles can thus be generated to study the sensitivity of different configurations to various regions within this three-dimensional sample space. Examples of these profiles are provided in Section 6.5 .

\section{Robustness to Size}

In addition to studying the effects of factors such as the level and type of transfer traffic and the industry structure in place, we can also examine the robustness of different configurations to changes in size. One of the primary issues related to size is whether the initial configuration selection (both in terms of concept and geometry) will be appropriate if the volume of passenger traffic increases to the extent that the airport will have to be expanded. For example, some configurations may perform well only for small volumes of traffic. If passenger volumes increase, the configuration concept itself may not be robust to changes in size. Moreover, even if the configuration concept itself is robust to changes in volume, the specific geometry selected initially may prevent successful expansion. Examples of how performance profiles can be used to study issues of size are provided in Section 6.6 . 


\section{4 - Robustness to Transfer Traffic}

This section illustrates some of the decisions that can be made using performance profiles when selecting an initial configuration of passenger buildings under uncertainty. In this case, we demonstrate how profiles can be used for decision support when the uncertainty lies in the level and type of transfer traffic that will exist at the airport, denoted by the parameters $P$ and $Q$ (defined in the previous section). As mentioned previously, each ordered pair $(P, Q)$ can be represented by a passenger mix object (Figure 6.5) and its attribute values can be used with the equations introduced in Chapter 5 to estimate the potential performance of a given passenger building configuration. By estimating potential performance for different possible geometries (over a range of values for $P$ and $Q$ ), we can determine which geometries and which configurations are most robust to changes in transfer traffic.

\subsection{1 - Centralized Configurations}

Figure 6.6 illustrates a characteristic performance profile for centralized (in this example, rectangular satellite) configurations for $G=24$. The values of $Q$ and $r$ are both held constant at .50 .

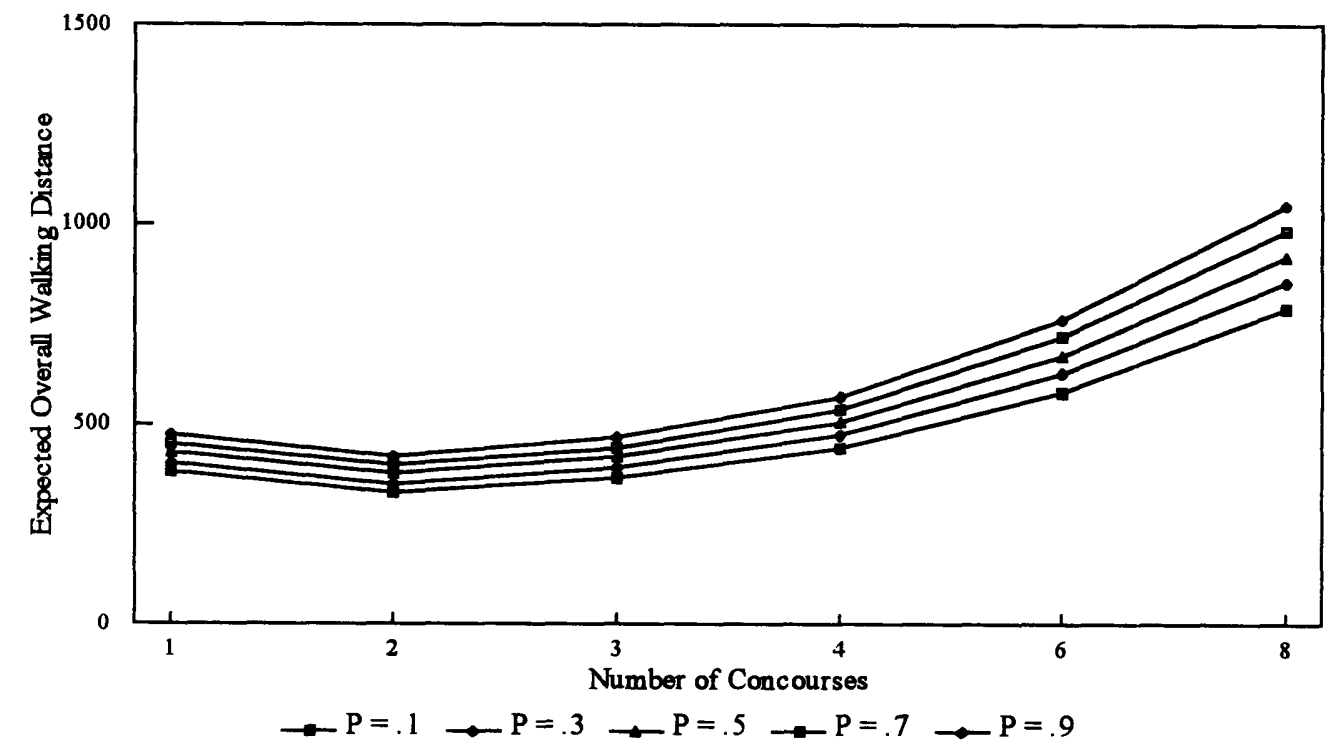

Figure 6.6 : Profile for Centralized Rectangular Satellite Configuration 
Similar to the centralized circular satellite configuration shown in Figure 6.1, the centralized rectangular configuration (Figure 4.6) shows an easily identified minimum for each value of $P$, in this instance at $n=$ 2 (i.e. $n=2$ dominates all other geometries). This situation is very different from the one shown in Figure 6.3, where the most appropriate geometry depends on the value of $P$. Additionally we see that, in general, the expected overall walking distance increases as the level of transfer traffic increases.

This behavior can also be seen if we disaggregate the overall walking distance curve into its three constituent components, as illustrated in Figure 6.7. Note that because of the way the data is maintained (i.e. in separate matrices for each passenger category), such a disaggregation is immediately available.

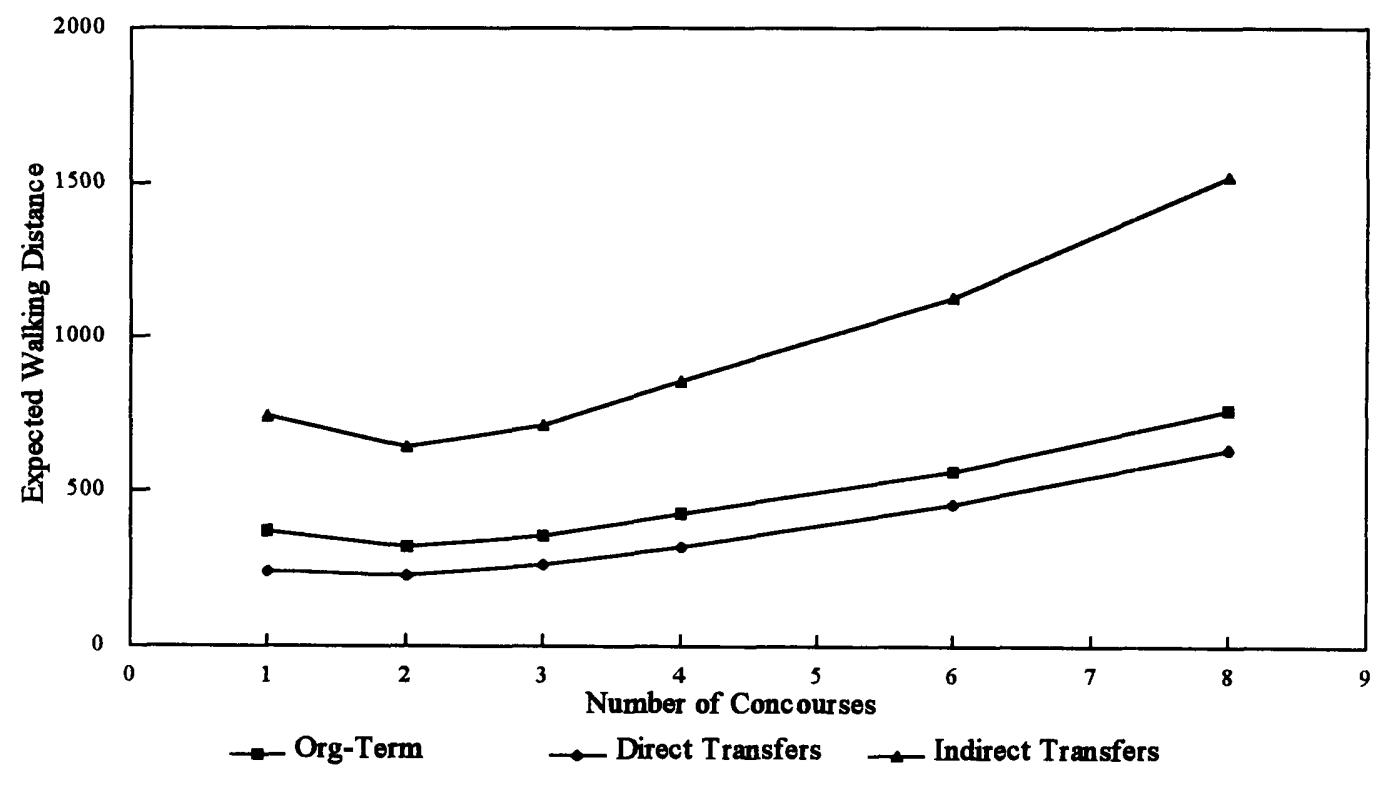

Figure 6.7 : Profile Lines for Individual Passenger Categories $(P=.5)$

In order to maintain mandatory separation standards between individual satellite concourses, the length of the connectors (between the centralized terminal block and the individual concourses) is derived from Equations 4.7 and 4.8. Between the geometries for $n=1$ and $n=2$, however, the connector length remains the same, and thus the expected walking distances (for each category) decrease due to the reduction in the length of the concourses (as $g$ decreases). 
As the number of concourses increases beyond two, however, the connector lengths become the principal determinants of walking distances, and the expected distances for all three passenger categories continue to increase as $n$ increases. Indirect transfer passengers are most affected by this increase in connector length, as it is assumed that they must return to the centralized terminal block (between arrival and departure) for intermediate services. Similar behavior (but with different numerical values) is evident for all the types of centralized satellite configurations in Table 6.5.

\subsection{2 - Decentralized Configurations}

A much different behavior is displayed for decentralized satellite configurations, as evidenced by the performance profiles shown in Figure 6.8 for a decentralized rectangular satellite concept.

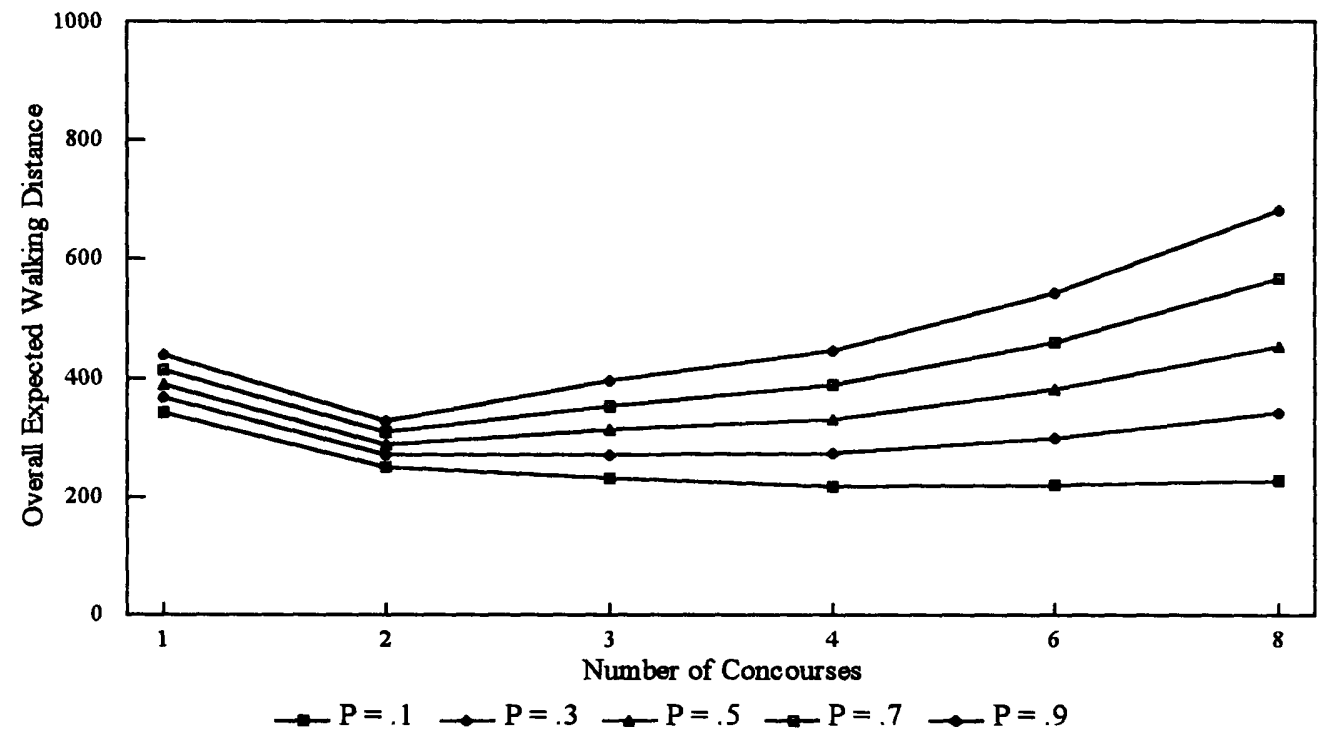

Figure 6.8 : Profile for Decentralized Rectangular Satellite Configuration

In this case (as in Figure 6.3), the convexity of the overall expected walking distance curve actually changes for different values of $P$. When $\mathrm{P}$ is small $P$ (i.e. a high proportion of originating and terminating traffic), the most appropriate geometry is one with many small individual concourses. Thus 
for $P=.1$, the geometry which minimizes the overall expected walking distance is one which contains eight concourses, each with three gates.

When $P$ is large (i.e. a high proportion of transfer traffic), however, the most appropriate geometry is one which contains two concourses, each with twelve gates. In fact, for values of $P$ higher than .50 , the eight-concourse geometry ("optimal" for $P=.1$ ) performs worse than all those considered. Again, we can understand this behavior by examining the profile lines for the individual passenger categories (Figure 6.9).

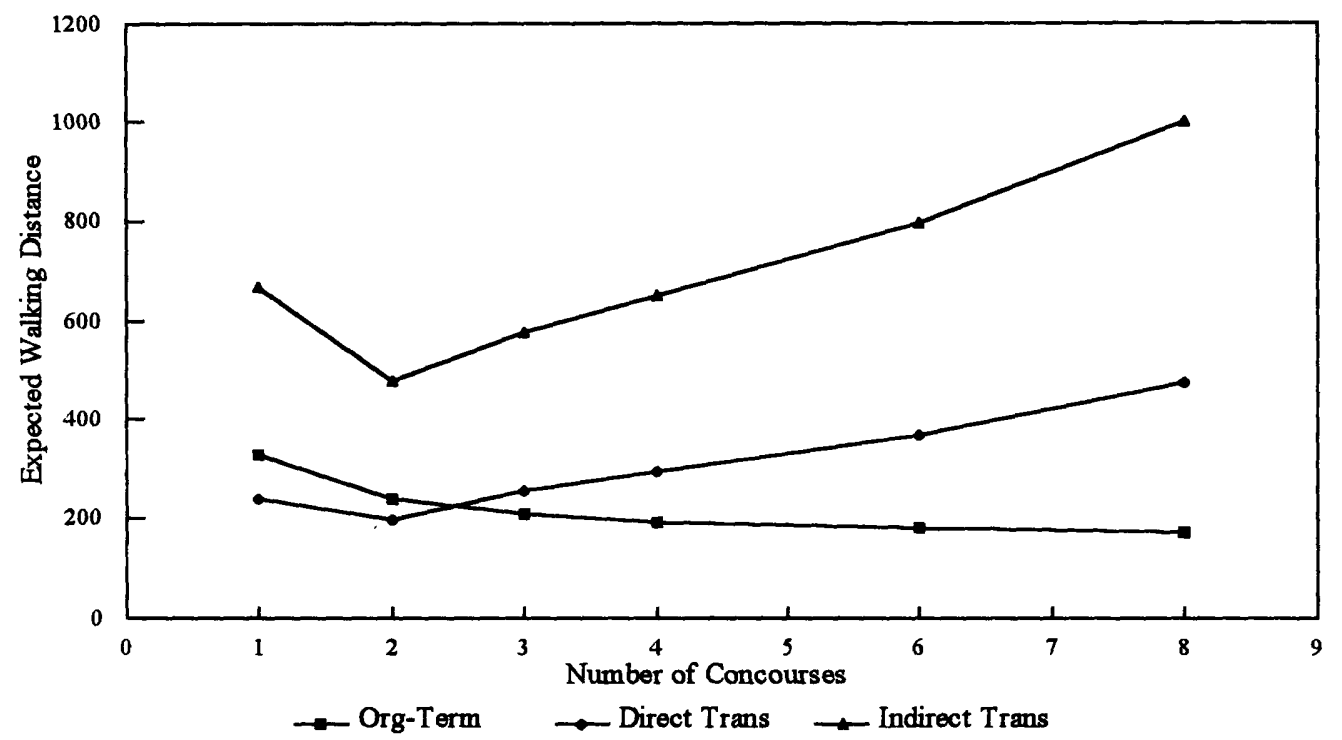

Figure 6.9: Profile Lines for Individual Passenger Categories $(P=.5)$

It can be seen that the profile line for originating and terminating passengers suggests that better performance is obtained by having more concourses with fewer gates, whereas the lines for both types of transfer passengers suggest that the most appropriate geometry is the one containing only two concourses.

Selecting the most robust geometry in terms of transfer traffic (i.e. the one that performs reasonably well over a wide range of $P$ ) thus depends on the uncertainty involved in the passenger mix forecast. If we are certain the level of transfer traffic will never exceed 10 percent, then the most appropriate geometry is $n=8, g=3$. If, however, there is uncertainty in our forecast, we can approach the selection process from a decision analytic perspective. In this case, the "risk" we are taking can be 
thought of as the difference, or "spread" between the highest and lowest walking distance estimates for the selected geometry. A possible decision tree is shown in Figure 6.10.

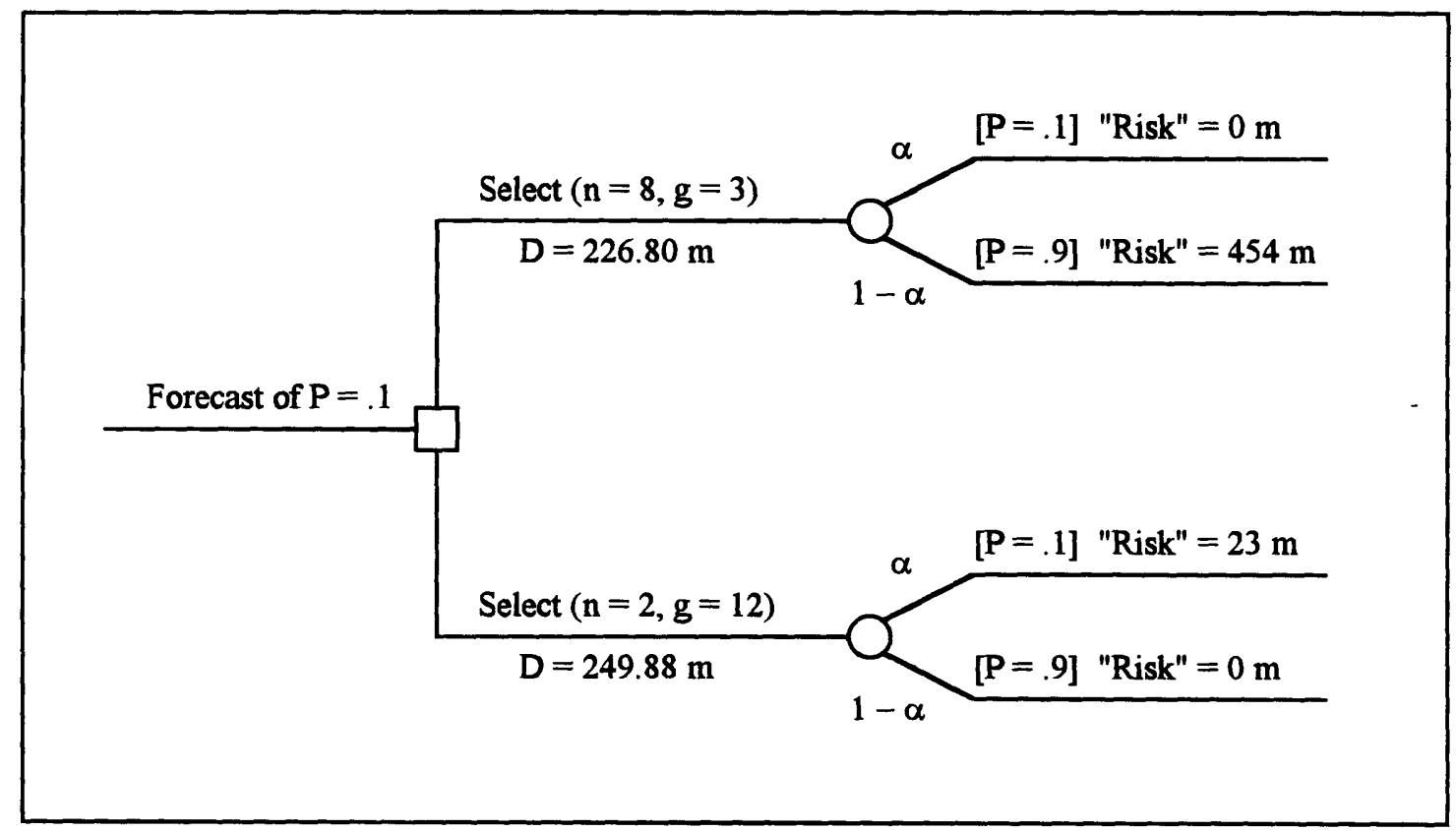

Figure 6.10 : Decision Tree for Selecting Most Robust Geometry

For simplicity, let us assume that two possible levels of transfer traffic are possible $(P=.1$ and $P=.9$ ), and that the probability each will actually materialize is $\alpha$ and $1-\alpha$, respectively. For a forecast of $P=.1$, we can select either the "optimal" ( $n=8, g=3)$ geometry or the "suboptimal" $(n=2, g=12)$ geometry. If we select the "optimal" geometry for the specific input data used, the risk we assume is that the actual overall expected walking distance will be $681.20 \mathrm{~m}$ (if $P=.90$ materializes), which is 454 meters higher than $226.80 \mathrm{~m}$. If, on the other hand, we select the "suboptimal" geometry, the risk we assume is only 23 meters (if $P=.1$ materializes).

If we use (again for simplicity) a linear utility function for passenger convenience as measured by walking distances, we can show that the "indifference $\alpha$ " between the two geometries is approximately .95 by equating the expected values of the risks, or

$$
0(\alpha)+454(1-\alpha)=23(\alpha)+0(1-\alpha) \text { when } \alpha \cong .95
$$


Thus, we must be more than 95 percent confident that our forecast of $P=.1$ is correct in order to select the "optimal" geometry.

\section{Considering Other Measures}

As mentioned previously, the expected overall walking distance or "first moment" of the walking distance distribution is only one metric by which we can compare different geometries when trying to select the most robust. Other measures, such as those described in Chapter 5, can provide the planner with additional information upon which to base his or her decision. Figure 6.11 illustrates a performance profile for the standard deviation of walking distances for the decentralized rectangular satellite configuration.

In contrast to the performance profile for expected walking distances, the profile line for low levels of transfer traffic $(P=.1)$ indicates that the standard deviation is minimized in the two-concourse, twelve gate geometry. The profile line for high levels of transfer traffic $(P=.9)$ demonstrates similar behavior. Thus, if our objective is to minimize the variability of walking distances (as measured by the standard deviation), we would select the $(n=2)$ geometry independent of the forecast for the level of transfer traffic.

Figure 6.12 illustrates the performance profile for the probability that a randomly selected passenger will have to walk a distance greater than the excess threshold (in this case, 350 meters). For low levels of transfer traffic, the most significant reduction in the "excess probability" occurs between geometries having one and two concourses. For geometries with more than two concourses, the distance profile line for the dominant passenger category (i.e. originating and terminating) is slowly decreasing (Figure 6.9) and thus the excess probability curve reflects only a small likelihood of those passengers having to walk long distances. For high levels of transfer traffic, the sharply increasing curves of the direct and indirect transfer passengers dominate the overall curve, and the excess probability curve remains fairly high (approximately .60). Thus, there are no significant advantages to decentralizing 
beyond two concourses when considering potential performance in terms of the probability of excess walking.

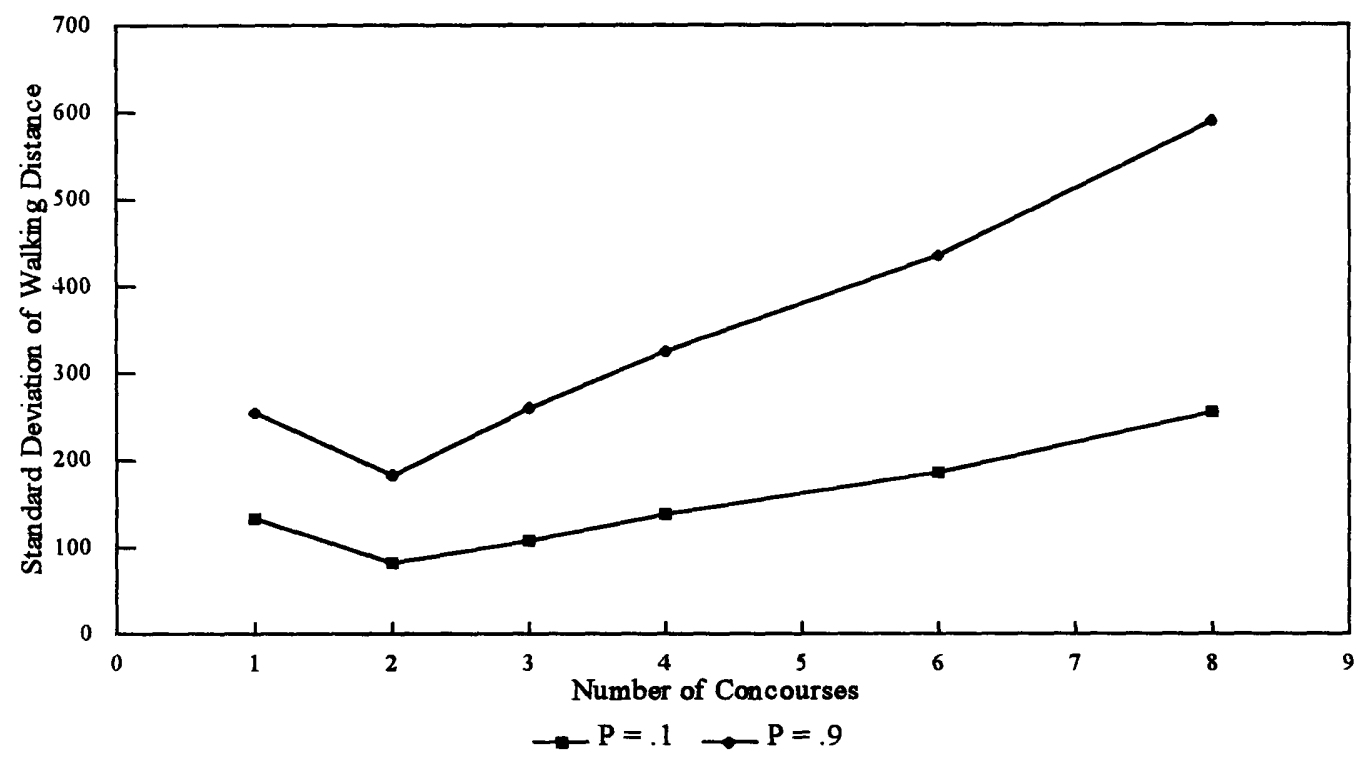

Figure 6.11 : Performance Profile of Standard Deviation

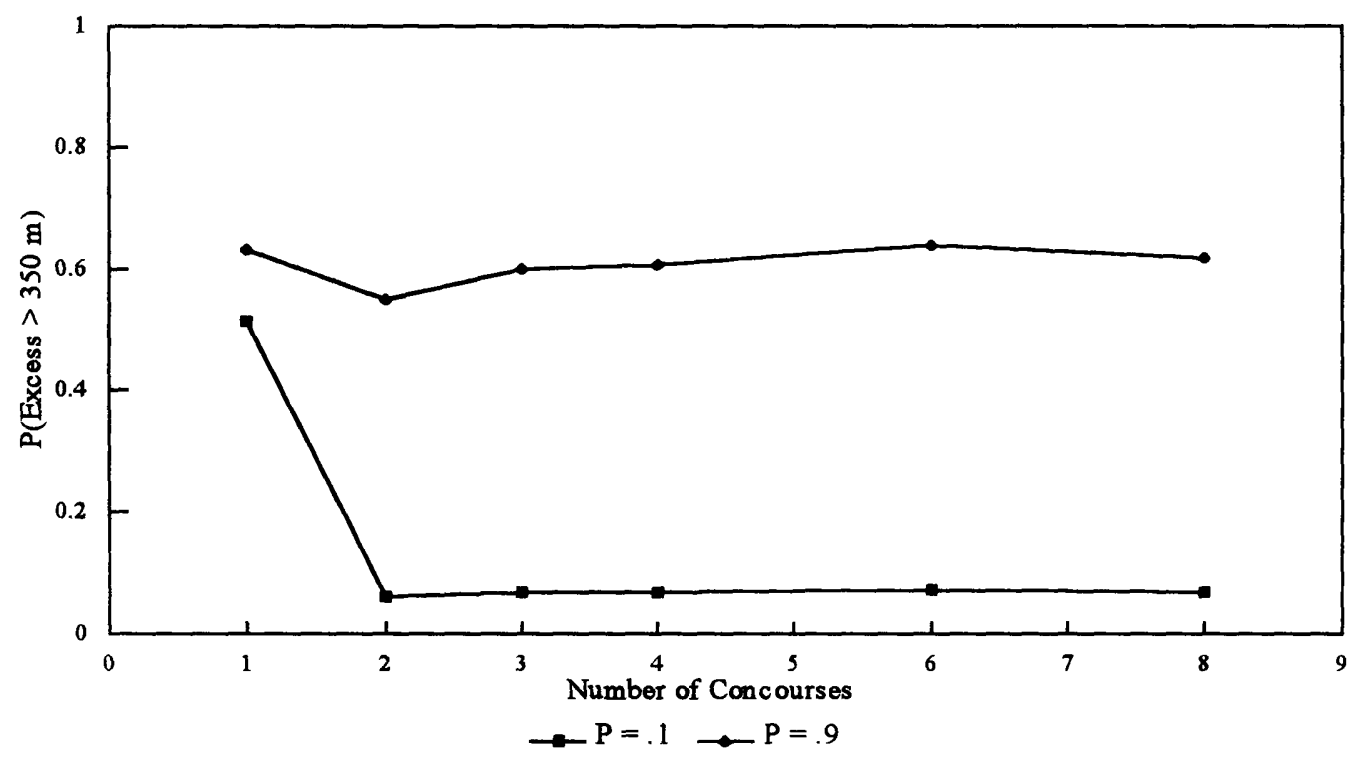

Figure 6.12: Performance Profile of Probability of Excess Walking 


\subsection{3 - Selecting an Initial Configuration}

The previous sections have illustrated specific examples of Step One in the selection of the initial configuration; namely, identifying the most appropriate or robust geometry (for a given configuration) over a range of conditions. Note that the techniques we have illustrated are independent of the particular criteria used. The methodology is sufficiently general that any criterion, if it can be modeled quantitatively, can be used to generate a performance profile for a range of different conditions. We have already seen that in some cases, selecting the most robust geometry is not as straightforward as simply choosing the value of $n$ which minimizes a particular metric (such as the overall expected walking distance). Due to the uncertainty of forecasts, it may be more appropriate to select a geometry which minimizes the variability of walking distances, or the risk of passengers having to walk excessively long distances.

In Step Two of the selection process, we consider the most robust geometry of each configuration concept and select the most robust concept type for a range of different conditions. To illustrate this technique, consider the input data given in Tables 6.7 and 6.8. We can use these data to characterize the different configuration concepts (as described in Chapter 4) for each of the geometries listed in Table 6.5. From these characterizations, we can generate performance profiles for each configuration concept, select the most robust geometry based on some criterion (minimizing overall expected walking distances, for example), and then select the most robust concept among the most robust geometries.

Figure 6.13 illustrates the results of this process when considering the entire sample space of the parameters $P$ and $Q$ (the level and type of transfer traffic), for configurations with $G=24$ gates. For the specific input data used, two concepts provide the most robust performance as measured by overall expected walking distances. For low levels of transfer traffic, the decentralized gate arrival configuration (Figure 4.9) performs well, due to the short distances between the access/egress points and departure/arrival gates for the dominant passenger category (i.e. originating and terminating passengers). 


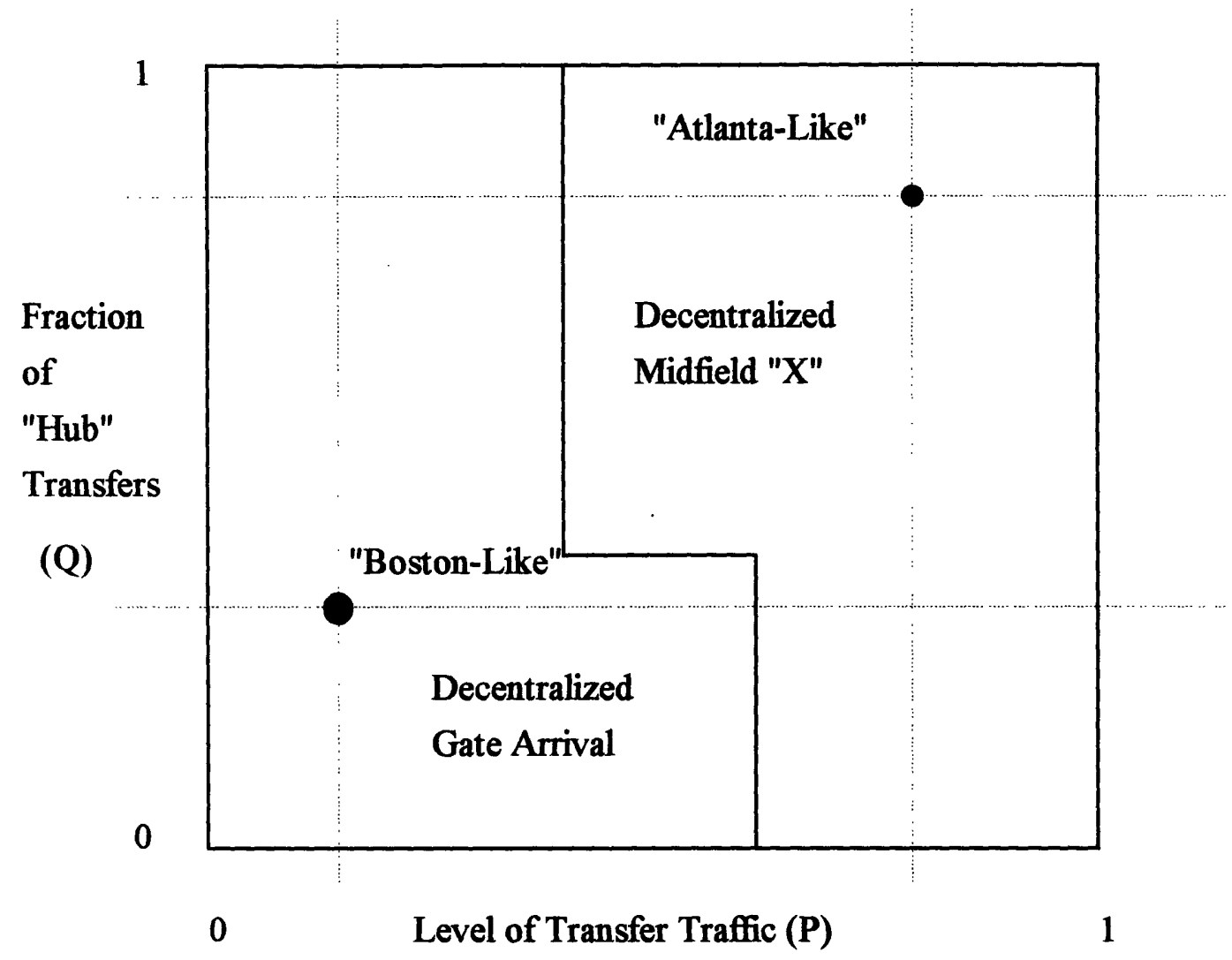

Figure 6.13 : $P$ vs. $Q$ "Best of the Best" Tableau for Walking Distances Only $(G=24)$ 
For higher levels of transfer traffic, the decentralized midfield " $\mathrm{X}$ " configuration (Figure 4.13) performs best of those considered. Walking distances for the dominant passenger category (now transfers) are minimized when gates are arranged as close as possible to the entrance point of the concourse. The "X" shape, among those considered, accomplishes this objective. Note, however, that for low values of $Q$, (i.e. a high level of indirect transfers), the "breakpoint" for the level of transfer traffic between the two configurations increases. For indirect transfers who must return to the terminal block for intermediate services, the increased distances of the connectors out to the X-shaped concourses make the gate arrival concept more desirable (for the specific data used).

As an example of how this analysis could be used at the planning level, let us assume that designers are considering the construction of a 24-gate airport at a greenfield site, with characteristics similar to those at Atlanta / Hartsfield (presently the largest hub for Delta Air Lines). As such, we would expect both the level of transfer traffic $(P)$ and the fraction of transfers able to make direct or "hub" connections $(Q)$ would be high (see point on Figure 6.13). The tableau suggests that the decentralized midfield " $\mathrm{X}$ " configuration would provide the most robust performance for the new site (in terms of minimizing overall expected walking distances). Even if there existed some error in the forecast of either parameter, the decision regarding the initial configuration would remain the same.

A similar analysis can be performed on a "Boston-Like" point meant to represent a situation similar to that at Boston / Logan Airport (see point on Figure 6.13). At an airport such as Boston / Logan, the level of transfer traffic $(P)$ would be significantly lower than at one similar to Atlanta / Hartsfield, and those passengers making connection would be more likely to require intermediate services. Thus the probability that a transfer passenger will be able to make a direct or "hub" connection would also be lower. Under such conditions, the tableau would recommend a decentralized gate arrival configuration, due primarily to the high level of originating and terminating passenger traffic expected. 


\section{5 - Robustness to Industry Structure}

Like the parameters $P$ and $Q$, the parameter $r$ (the fraction of direct transfers certain to depart from their arrival concourse), can be varied in order to study the robustness of different configurations (and geometries within a particular configuration) to industry structure or carrier dominance at an airport. As mentioned previously, high values of $r$ do not necessarily translate into a strong carrier presence; however, the parameter does in some sense provide an idea of how the gates in the airport are shared (i.e. what stand management policies are in place), and is used here for illustrative (and further proof of concept) purposes.

\section{Characteristic Behavior of Performance Profiles}

Figure 6.14 illustrates a characteristic performance profile for selecting the most robust geometry for a given configuration, in this case for a centralized parallel pier configuration (Figure 4.2) with $G=24$ gates, $P=.9$, and $Q=1.0$. For low values of $r$ (for which all gates within the airport become equally likely for departure), the most appropriate geometry for the given configuration is one with only a single concourse. Due to the uncertainty of a direct transfer passenger's departure gate, the geometry that minimizes walking distances (for the specific input data used) is one in which all gates are located within the same concourse. As the number of concourses increases, so does the probability of having to make an interterminal transition. In this case, the added distance of having to transfer between passenger buildings increases the overall expected walking distance each time $n$ is increased.

For high values of $r$, the opposite case is true. If direct transfer passengers are certain to depart from their arrival concourse, then their walking distances will be minimized when individual concourses are very small (due to the absence of any interterminal transitions). Note, however, that the overall expected walking distance estimate will be affected only to the extent that direct transfers make up a significant portion of the total traffic. In our example, the level of direct transfer traffic is high $(P=.9, Q$ $=1.0$ ). For lower levels of $P$ (and $r=1.0$ ), there will exist a more balanced trade-off between the 
increased walking distances for originating and terminating passengers (due to longer distances out to the more remote concourses), and the decreased walking distances for direct transfers due to smaller individual concourses. These trade-offs could readily be examined using performance profiles for the individual passenger categories, such as those previously shown in Figures 6.7 and 6.9 .

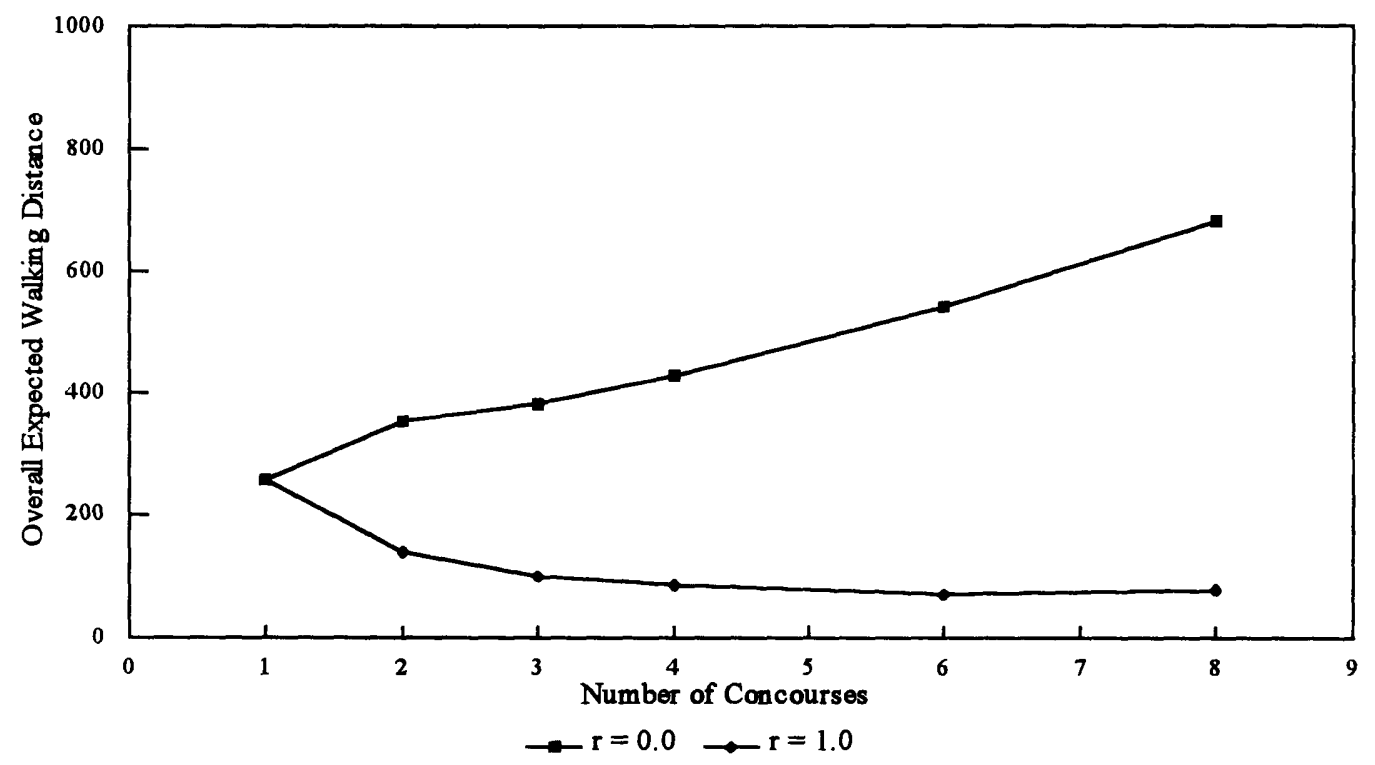

Figure 6.14 : Performance Profile for Centralized Parallel Pier Configuration

\section{Considering Other Measures}

Figures 6.15 and 6.16 illustrate performance profiles for the standard deviation of walking distances and the probability of excess walking (above $350 \mathrm{~m}$ ), respectively. For both low and high values of $r$, the standard deviation generally increases as $n$ increases above three. For low values of $r$, the behavior of the estimate reflects the increased distances walked by those direct transfer passengers making interterminal transitions. For high values of $r$, the estimate is driven mostly by the distances of originating and terminating passengers, as they must walk to smaller concourses farther away from the centralized terminal block. 


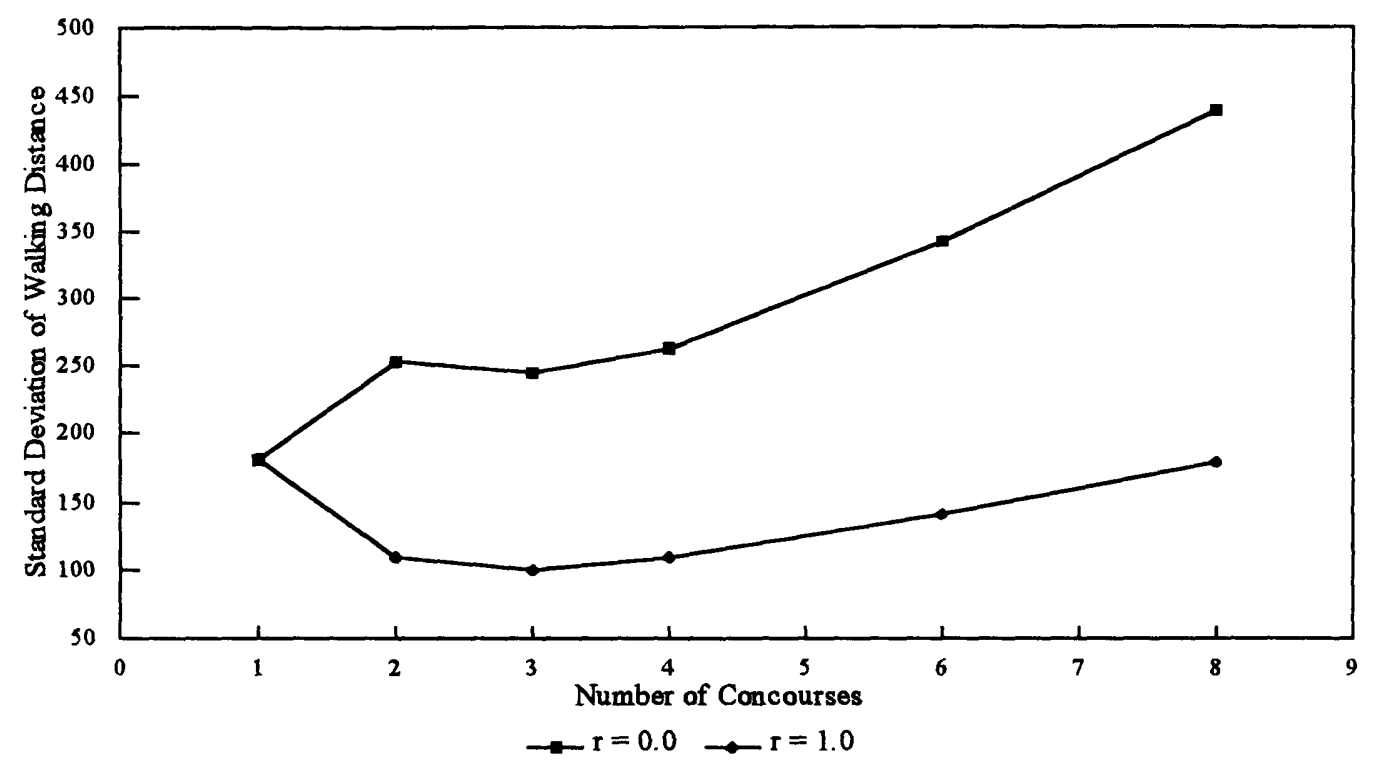

Figure 6.15 : Performance Profile of Standard Deviations

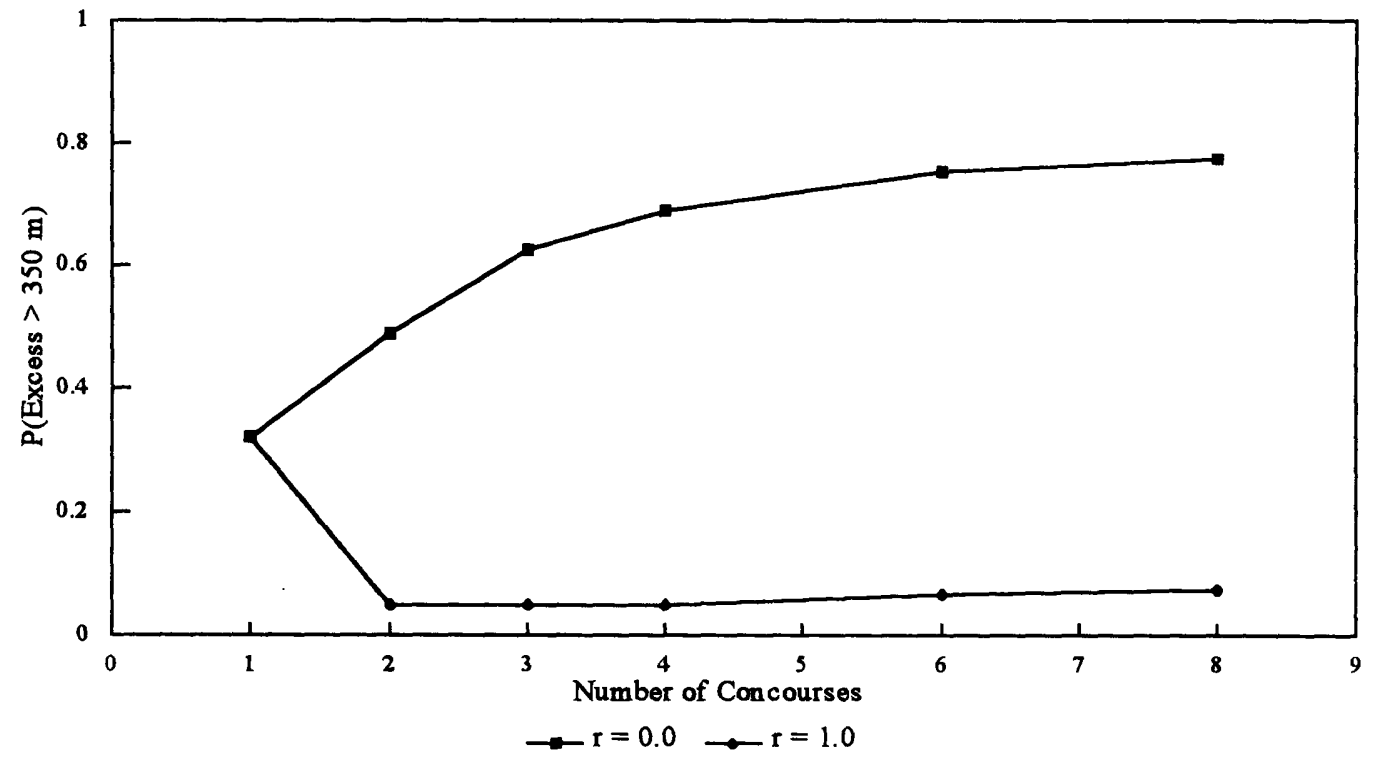

Figure 6.16: Performance Profile of Probability of Excess Walking 
The profile lines for the probability of excess walking behave similarly to the profile lines for the overall expected walking distance (Figure 6.14). For low values of $r$, the probability increases asymptotically to approximately .80 . The remaining 20 percent are composed of those direct transfers who wind up departing from their arrival concourse, as well as those originating and terminating passengers who depart from gates within concourses still located inside the 350 meter threshold "radius". For high values of $r$, the probability of excess walking is small (above $n=1$ ) because the only passengers required to walk distances above 350 meters are those originating and terminating passengers departing and arriving from remote concourses. Recall that for this example the level of transfer traffic is assumed to be high $(P=.9)$. Thus the small fraction of originating and terminating passenger traffic does not greatly affect the overall estimate.

\section{Selecting an Initial Configuration}

In order to select a configuration which is robust to changes in industry structure, we would like to generate a tableau similar to the one illustrated in Figure 6.13 (for selecting a configuration which is robust changes in the level of transfer traffic). Unlike the parameter $Q$, however, the parameter $r$ depends both on the specific geometry being considered and the industry structure in place. Therefore it cannot be used as a direct input across all geometries (for a specific configuration).

For example, if there were two dominant carriers at an airport and each operated exclusively out of their own dedicated concourse (for a geometry where $n=2$ ), we might expect the value of $r$ to be close to 1.0. For an equivalently sized airport (in terms of the number of gates) with the same two carriers each operating out of two concourses (thus $n=4$ ), we might expect the value of $r$ to be closer to 0.5 (depending on the stand management policies in place). Thus, as mentioned previously, the value of $r$ for a specific configuration depends both on the number of individual concourses and on the number of "effective" competitors at the airport, which we denote $C$. Note that $C$ can be thought of as one greater than the number of dominant carriers, where the remaining competitors constitute a single "effective" one for passenger distribution purposes. 
Using this definition of $C$, we can determine different values of $r$ for each of the possible geometries listed in Table 6.6, depending on the specific assumptions made regarding the distribution of gates to effective competitors. Examples of some possible assignments of competitors to concourses, and the resulting values of $r$ are summarized in Figure 6.17 and Table 6.9.

Given the values for $r$ in Table 6.9, it is possible to explore the robustness of different configuration types to changes in industry structure, this time as measured by the number of effective competitors. Moreover, we can vary the level of transfer traffic, $P$, (while holding $Q$ constant) to obtain a tableau similar to Figure 6.13 , this time plotting $P$ vs. $C$. The results of an analysis using the objective "to minimize overall expected walking distances" is illustrated in the tableau shown in Figure 6.18.

As in the $P$ vs. $Q$ tableau, the decentralized gate arrival configuration performs best under low levels of transfer traffic due to the small distances walked by originating and terminating passengers. As $P$ increases, however, the best performing configurations become the decentralized parallel pier (Figure 4.10) and the decentralized midfield " $\mathrm{X}$ " (Figure 4.13) concepts (depending on the number of competitors, C.

To understand the results of this tableau more fully, consider the illustrations shown in Figure 6.19 corresponding to the most appropriate geometries for the ordered pairs $(P, C)$ of $(.70,1)$ and $(.70,4)$. For ordered pair $(.70,1)$, a single "effective" competitor faces a passenger mix forecast that is predominantly transfer traffic. Under this condition, the overall expected walking distance is minimized with a single " $\mathrm{X}$ "-shaped concourse [Figure 6.19(a)], thus eliminating lengthy transfers between buildings. Even with two effective competitors, operating out of a single "X"-shaped concourse still minimizes the overall expected walking distance (for the input data given).

As $C$ increases above two, however, the decentralized parallel pier concept becomes more appropriate [Figure $6.19(\mathrm{~b})]$. For the ordered pair $(.70,4)$ the most appropriate geometry is one in which $n=4$, i.e. each of the effective competitors operates out of its own individual concourse. The situation is very similar to having a single, midfield " $\mathrm{X}$ " concourse with each competitor operating out of a single arm of the "X"; however, the decentralized parallel pier concept provides the small fraction of originating and terminating passengers with shorter walking distances. 
Once again consider the situation in which planners are selecting an initial configuration for a 24-gate airport based on robustness (this time) to industry structure. The point labeled "Atlanta-Like" (Figure 6.17) represents the situation currently at Atlanta/Hartsfield, in which there exists a high level of transfer traffic and the industry structure is characterized by two "effective" competitors, Delta Air Lines and the "Other Airlines". Under such conditions, the tableau recommends a centralized midfield "X" configuration, for the reasons mentioned previously.

Consider also a situation which could be described as "Chicago/O'Hare-Like", which can again be characterized by a high level of transfer traffic (though not as high as Atlanta); however, this time there exists at least three "effective" competitors (United, American, and "Others"). For a 24-gate airport which would operate under these conditions, the tableau recommends a decentralized parallel linear configuration, allowing each competitor at least one of its own dedicated concourses.

Note, however, that these conclusions are based on very specific data and should not be generalized to all situations. Figure 6.18 is provided as an example of the type of analysis that can be performed, rather than as a reference source for selecting the unqualified "best configuration" concept. In the next section, we discuss how analyses similar to these can be applied to making decisions regarding robustness to size (i.e. designing for expansion).

Table 6.9 : Possible Values of $r$ Given $C$ and $n$

$\begin{array}{lrrrrrr} & \underline{\mathrm{n}=1} & \underline{\mathrm{n}=2} & \underline{\mathrm{n}=3} & \underline{\mathrm{n}=4} & \underline{\mathrm{n}=6} & \underline{\mathrm{n}=8} \\ \mathrm{C}=1 & 1.000 & .500 & .333 & .250 & .166 & .125 \\ \mathrm{C}=2 & 1.000 & 1.000 & .556 & .500 & .333 & .250 \\ \mathrm{C}=3 & 1.000 & .833 & 1.000 & .625 & .500 & .333 \\ \mathrm{C}=4 & 1.000 & 1.000 & .833 & 1.000 & .556 & .500\end{array}$




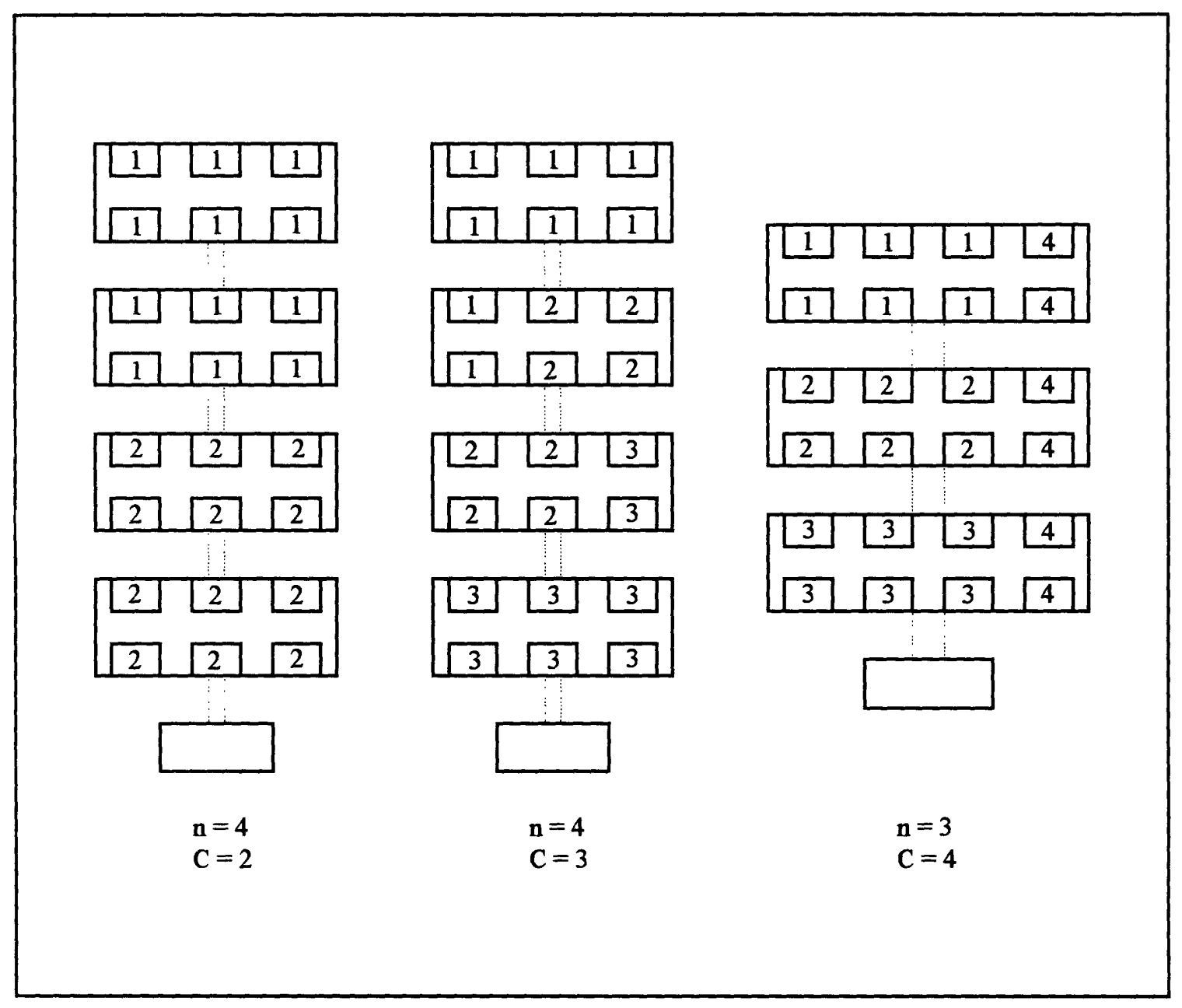

Figure 6.17 : Some Possible Assignments of Competitors to Concourses 


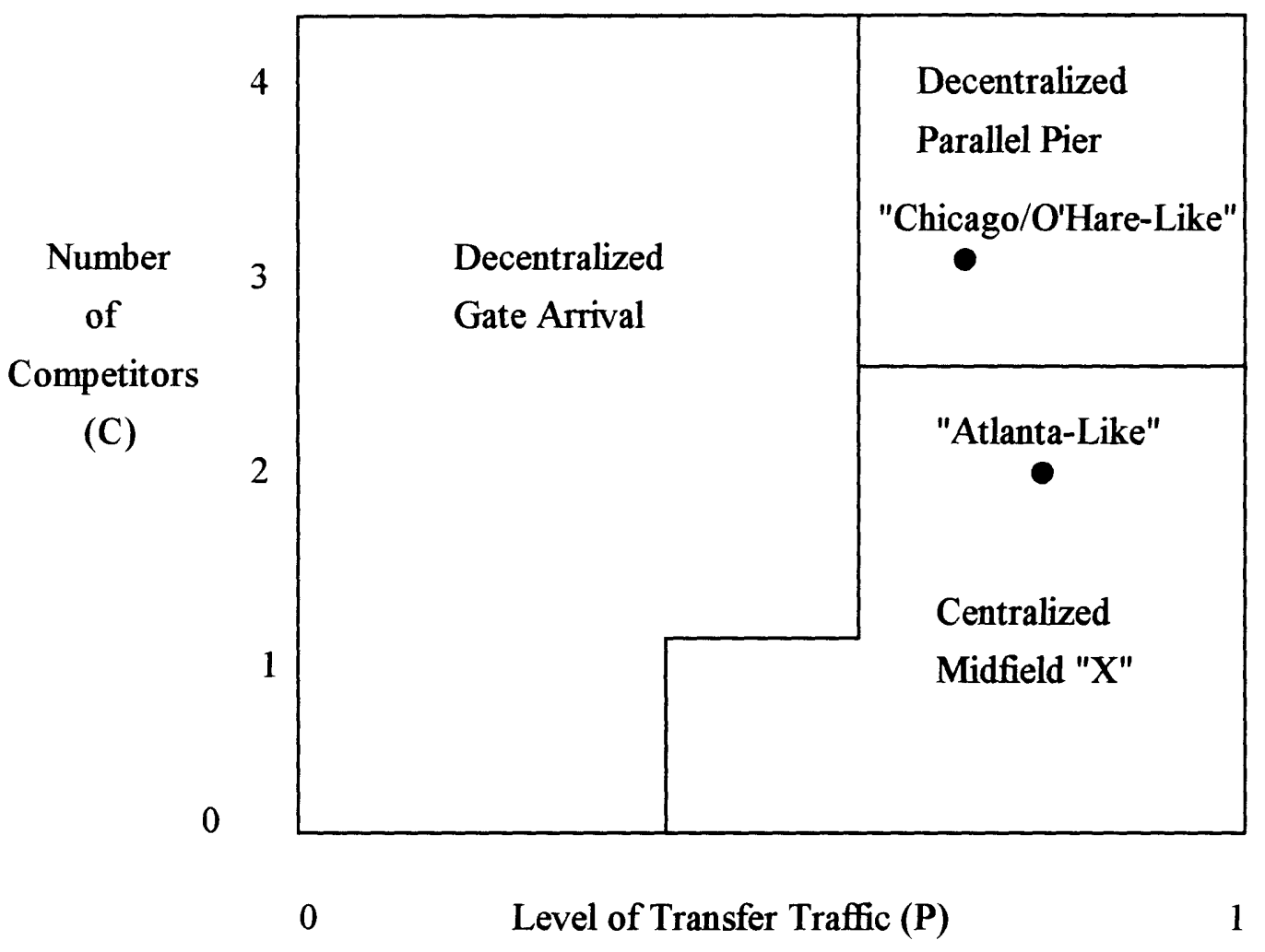

Figure 6.18: $P$ vs. $C$ "Best of Best" Tableau for Walking Distances Only $(G=24)$ 


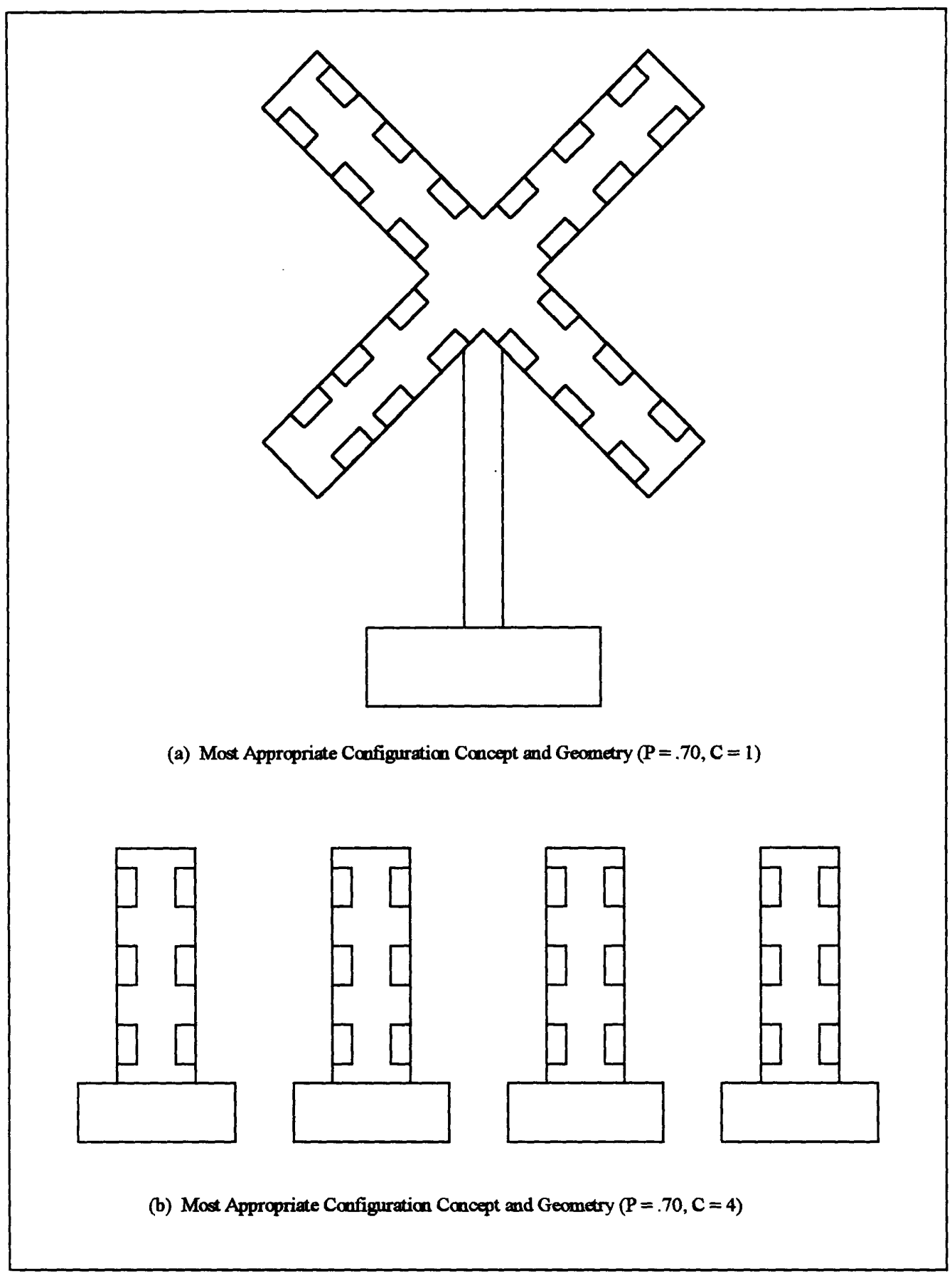

Figure 6.19: Graphical Representation of $(P$ vs. $C)$ Tableau Results 


\section{6 - Robustness to Size (Design for Expansion)}

Thus far the selection of the initial configuration has been done for a constant (and given) number of total gates, $G$. In fact, however, the total gate position requirement for an airport is uncertain (see Chapter 2), and selecting the most robust initial geometry and configuration based on a single value of $G$ may produce an inappropriate decision if changes in traffic volume necessitate expansion. To prevent this problem, we might decide to select the most appropriate geometry and configuration for traffic volumes associated with long-term forecasts. Construction could then be accomplished in stages, expanding landside capacity via the number of concourses and gates as needed. One of the obvious "risks" involved in such a strategy, however, is that the long-term forecast is almost certain to be incorrect. Passenger volumes may either exceed expectations or never materialize.

We can again approach the problem of selecting an initial geometry and configuration (under the uncertainty of future passenger volumes) from a decision analytic perspective. Once again we can define the "risk" of our decision as the difference in the selected performance metric between the most appropriate geometry (given perfect information regarding future passenger volumes) and the one selected for the short term. As an example, consider the decision of selecting the most robust geometry (to changes in size) for a given configuration -- a similar analysis could then be performed across configuration types.

Figures 6.20 and 6.21 illustrate two performance profiles for centralized, circular satellite configurations (Figure 4.5). Figure 6.20 shows the potential performance of the given configuration (as measured by overall expected walking distances) for $G=24$ gates, based on data provided in Table 6.7. Figure 6.21 shows the potential performance for the same configuration type containing $G=48$ gates. As discussed previously, the characteristic behavior of profiles for centralized configurations is independent of the level of transfer traffic. Thus the selection of the most robust geometry can be done simply by selecting the minimum value on any of the profile lines shown. In this case let us assume the particular forecast level of transfer traffic is $(P=.9)$ 


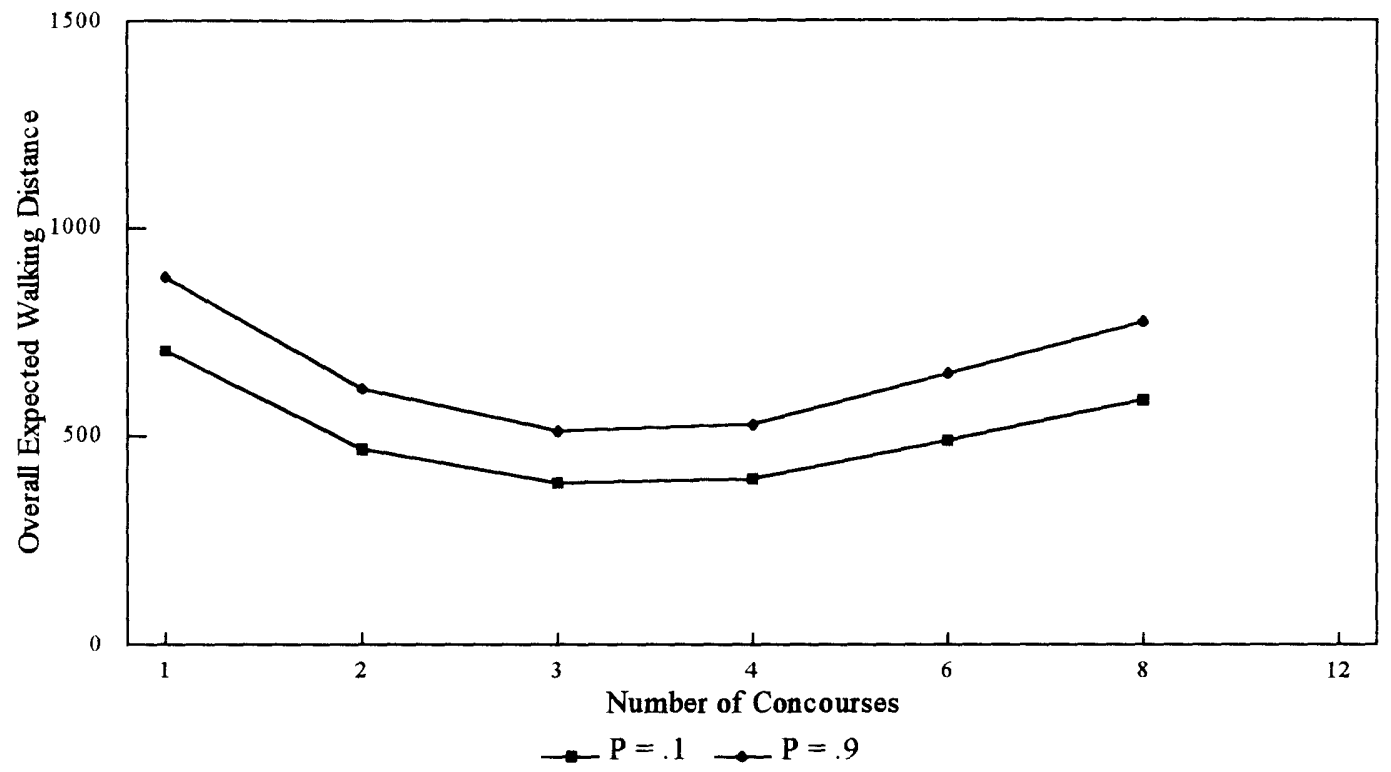

Figure 6.20: Performance Profile for Centralized Circular Satellite $(G=24)$

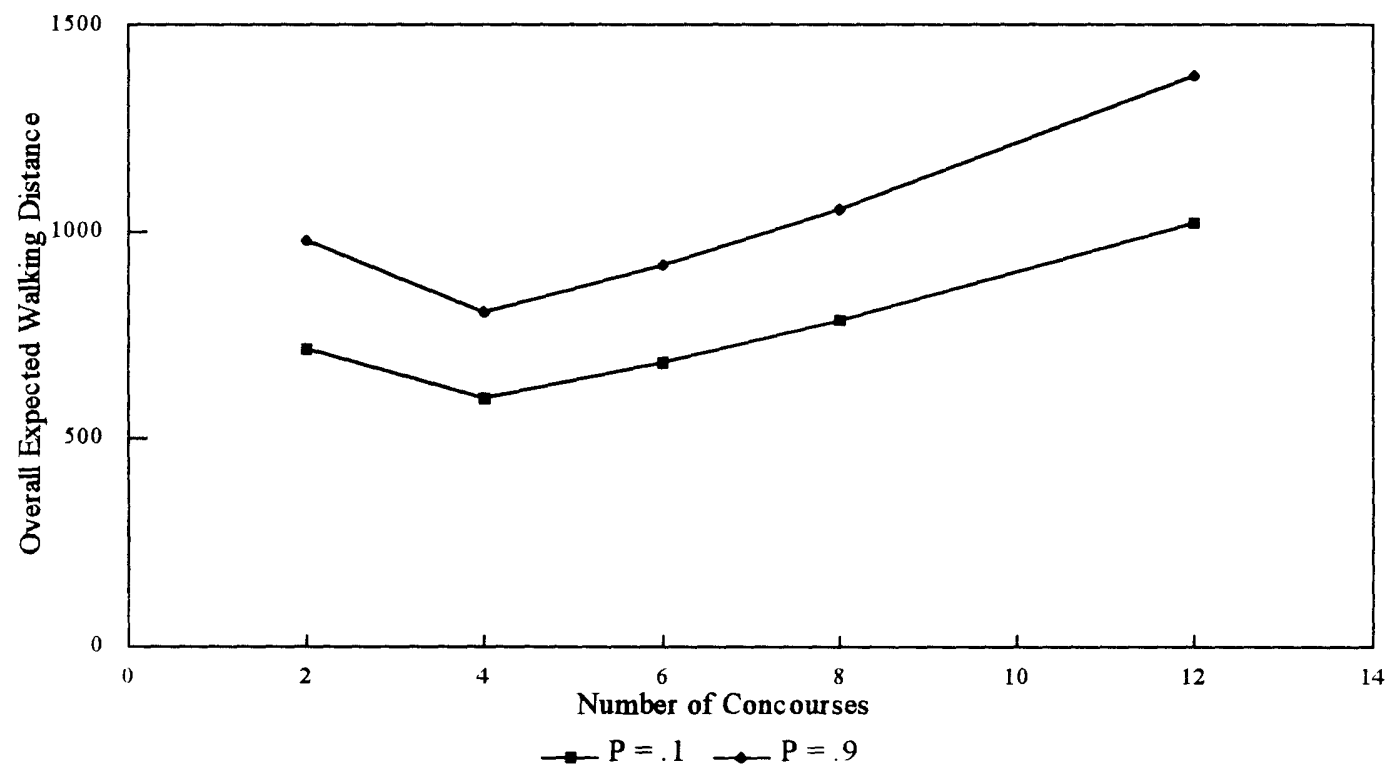

Figure 6.21 : Performance Profile for Centralized Circular Satellite $(G=48)$ 
Under the given assumptions, the most appropriate geometry for $G=24$ is one with $n=3$ concourses, each containing eight gates $(g=8)$. Let us further assume that if we select this geometry in the short-term, expansion to a 48-gate airport would be accomplished by adding three more 8-gate concourses. Initially selecting the most appropriate geometry for a configuration with $G=\mathbf{4 8}$ gates is equally straightforward -- the overall expected walking distance is minimized when $n=4$ concourses, each containing twelve gates $(g=12)$. In the short term, we assume that we would build only two of these 12-gate concourses to satisfy demand.

For illustrative purposes, we can represent the decision of selecting the most appropriate geometry under the uncertainty of future traffic volumes with a simple matrix shown in Figure 6.22 , where $D$ is the overall expected walking distance for the given geometry. For each decision we assume that if traffic volumes do not materialize, the selected short-term $(G=24)$ geometry will be sufficient (i.e. no intermediate values of $G$ are used). The decision tree representation for the matrix is illustrated in Figure 6.23.

The "risk" involved in both decisions is one of operating a suboptimal geometry, either in the short-term or in the long-term, depending on whether or not the long-term traffic forecast materializes. In this case, the absolute values of the risks are similar, so the decision may depend on factors other than overall expected walking distance. Similar decision trees could be generated for other performance metrics relevant to the decision, and "indifference $\alpha$ 's" calculated to determine the required confidence in the long-term forecast.

This chapter has focused on making decisions regarding the selection of an initial configuration from a passenger convenience perspective. As mentioned previously, however, the models and techniques we have demonstrated are entirely general and can be applied to many other aspects of potential airport performance. The next two chapters describe other applications of our object-oriented approach, using similarly structured data to measure potential configuration performance. In Chapter 7, we examine performance measures related to baggage and aircraft movement, and show how the selection of the initial configuration of passenger buildings might affect them. 


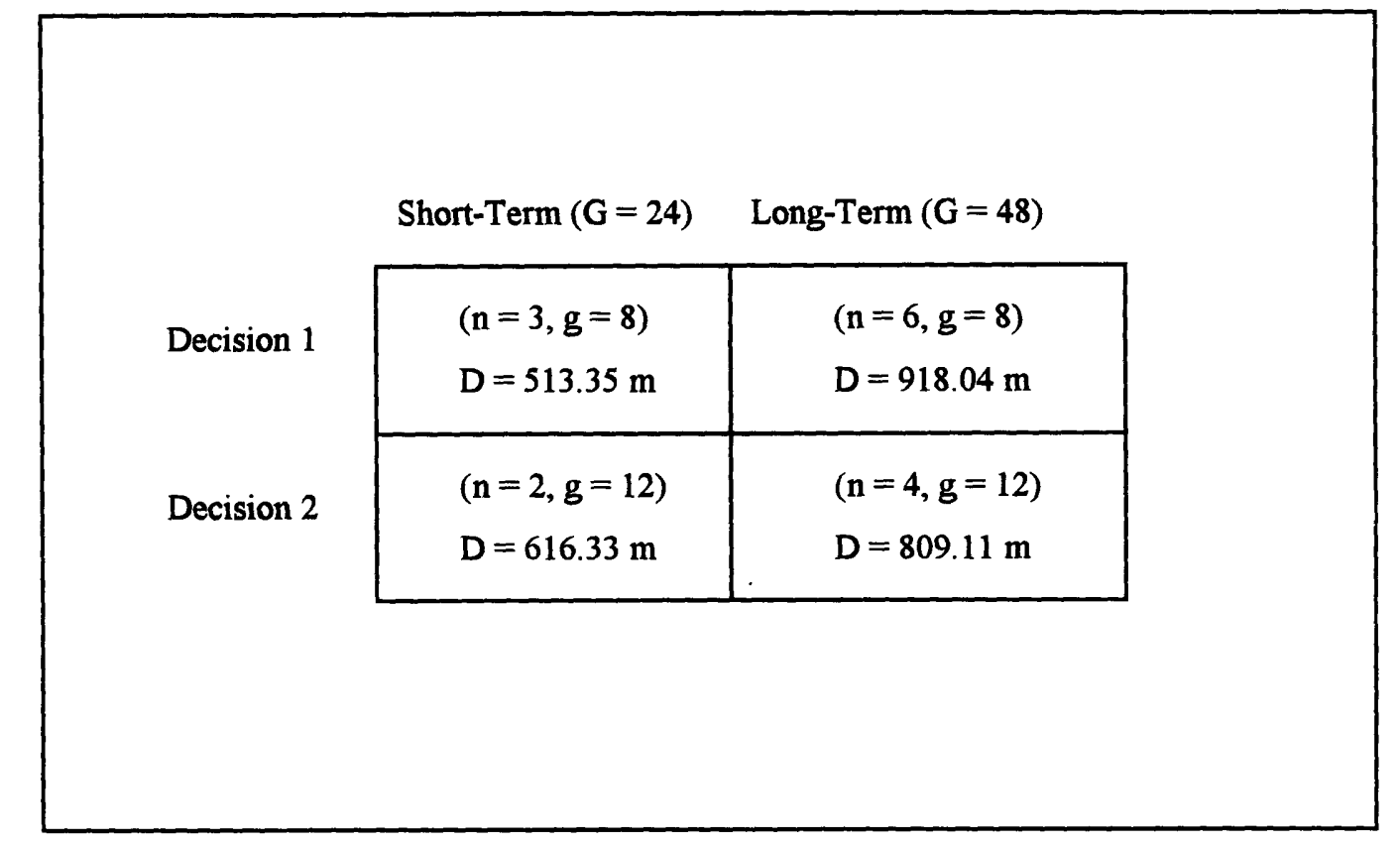

Figure 6.22: Simplified Decision Matrix for Expansion Decision

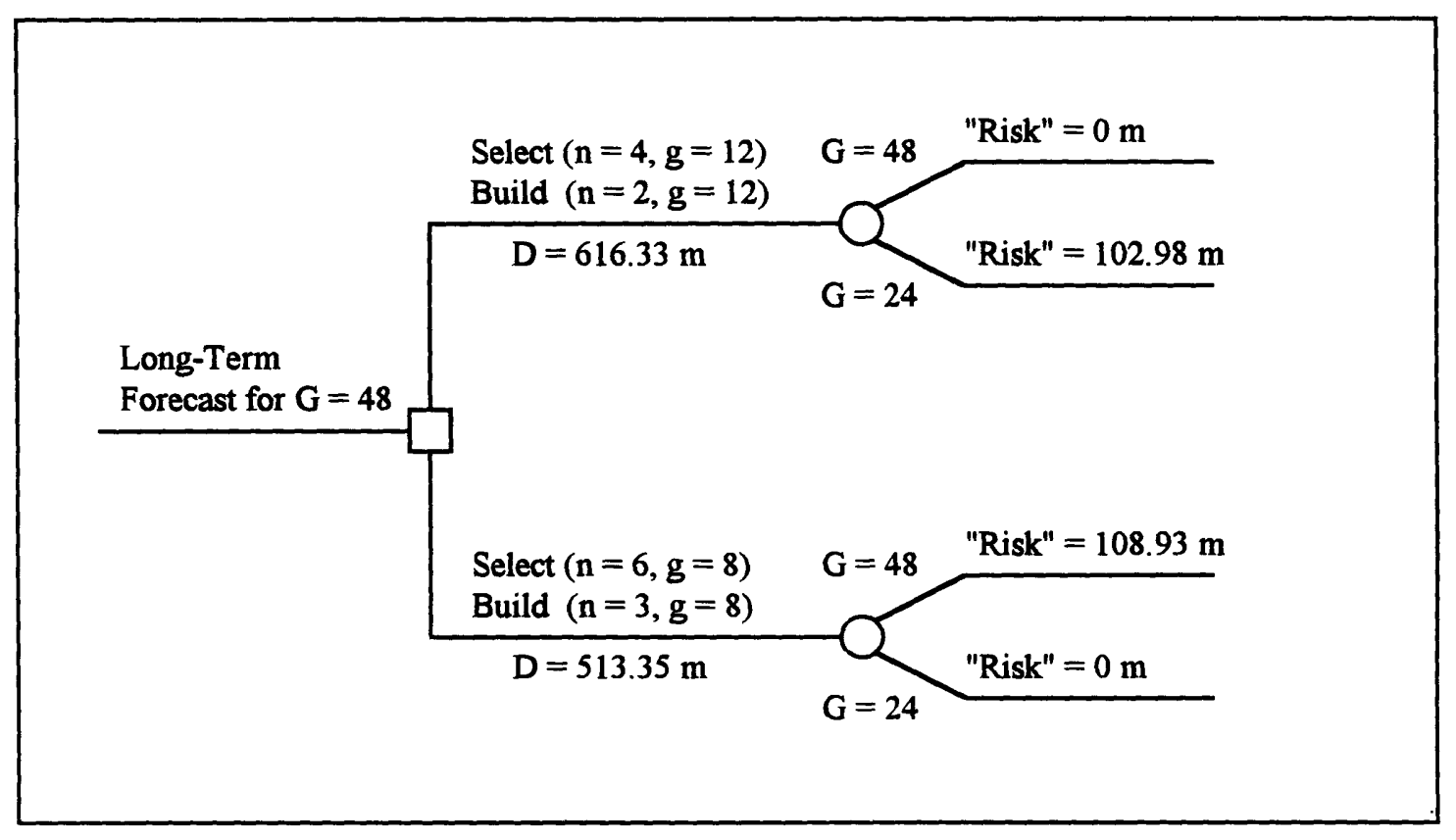

Figure 6.23 : Decision Tree for Selecting Most Robust Geometry (for Expansion) 


\title{
Chapter 7
}

\section{Estimating Potential Airport Performance}

\section{Part II : Baggage and Aircraft Measures}

\author{
Introduction
}

Chapter 5 demonstrated how well-known results of geometric probability could be used to estimate measures of potential configuration performance related to passenger convenience, namely passenger walking distances and overall travel times. Chapter 7 demonstrates how similar techniques can be used to estimate performance measures related to aircraft and baggage movement. Such measures are directly related to passenger convenience, as the longer passengers must wait onboard an aircraft or in front of a baggage claim carousel, the less "convenient" their perception of their airport experience is likely to be. Sections 7.1 and 7.2 discuss how the techniques introduced in Chapter 5 can be used to estimate expected baggage transfer times for a given passenger building configuration. Sections 7.3 and 7.4 apply similar techniques to develop a model which could help to estimate aircraft taxi times. 


\section{1 - Baggage Transfer Times}

Unlike passengers, baggage is not "inconvenienced" by traveling long distances or by having to transfer from one building to another. Rather, it is passengers who are inconvenienced when they must wait at a crowded baggage claim area or when their bags, destined for Chicago, somehow are sent to Tokyo. ${ }^{1}$ Ashford and Wright $[1979]$ note that :

Unlike most other modes, in air transport it is customary to separate passengers from their baggage during the line haul portion of the trip. This adds to the complexity of handling the air trip and seriously complicates the design of passenger terminals, since it is essential that the separation and reuniting of passenger and baggage be carried out with maximum efficiency and at an extremely high level of reliability. [p. 259]

Thus, transfer time (rather than distance) and reliability become important issues when assessing potential configuration performance in terms of baggage operations. Of these two issues, transfer times are directly influenced by the geometry of the passenger buildings [Robusté 1991b]. Although the reliability of baggage operations is at least in part affected by the geometry of passenger buildings, it is more likely to be influenced by issues such as the level of automation, the degree of redundancy in the system and the manpower methods in place.

Figure 7.1 illustrates the sequence of baggage flow throughout the airport system. Similar to the different passenger categories described in Chapter 4, baggage can be placed into different categories for the purposes of modeling transfer times. Typically these categories are :

- Inbound (Terminating) Baggage

- Outbound (Originating) Baggage

- $\quad$ Normal Transfer Baggage

- $\quad$ Ramp Transfer (Short Connection) Baggage

A brief description of each follows.

\footnotetext{
${ }^{1}$ Author's personal experience.
} 


\begin{tabular}{|c|c|c|}
\hline Inbound & & Outbound \\
\hline \multirow{2}{*}{$\begin{array}{l}\text { Unloading from } \\
\text { aircraft }\end{array}$} & Ramp & \multirow{2}{*}{$\begin{array}{l}\text { Carriage of baggage } \\
\text { to check-in }\end{array}$} \\
\hline & Transfer & \\
\hline \multirow{2}{*}{$\begin{array}{l}\text { Transport to } \\
\text { terminal airside }\end{array}$} & Normal & \multirow{2}{*}{$\begin{array}{l}\text { Check-in procedures } \\
\text { including tagging and } \\
\text { occasional weighing }\end{array}$} \\
\hline & Transfer & \\
\hline $\begin{array}{l}\text { Sortation -- loading } \\
\text { onto claim devices }\end{array}$ & & $\begin{array}{l}\text { Conveyance to } \\
\text { Airside }\end{array}$ \\
\hline $\begin{array}{l}\text { Conveyance to } \\
\text { reclaim area }\end{array}$ & & $\begin{array}{l}\text { Sortation and makeup } \\
\text { into aircraft loads }\end{array}$ \\
\hline $\begin{array}{l}\text { Presentation of baggage } \\
\text { to passengers }\end{array}$ & & $\begin{array}{l}\text { Transport of baggage } \\
\text { to planeside }\end{array}$ \\
\hline $\begin{array}{l}\text { Carriage from } \\
\text { reclaim area }\end{array}$ & & $\begin{array}{l}\text { Loading onto } \\
\text { Aircraft }\end{array}$ \\
\hline
\end{tabular}

Figure 7.1: Flow of Baggage ${ }^{2}$

${ }^{2}$ Adapted from : [Ashford et al. 1991], p. 180. 
Inbound Baggage

One sequence for inbound baggage is illustrated in the left column of Figure 7.1. First, baggage is unloaded from the aircraft onto trucks, which then transport the baggage to a sortation room typically located within the terminal. Baggage on wide-bodied aircraft is generally stored in containers, such as the LD-3, designed so that two containers fit side-by-side in the underfloor of the cargo hull on most large aircraft. Therefore, containerized baggage must also be unloaded from containers before being sorted. Once inbound baggage arrives at the sortation room, it is loaded onto reclaim (or display) devices so that passengers can retrieve it. Depending on the size of the airport, various forms of reclaim devices can be used such as linear counters, linear conveyors, carousels, and racetracks [IATA 1989].

\section{Outbound Baggage}

One sequence for outbound baggage is illustrated in the right column of Figure 7.1. Baggage not taken onboard is separated from the originating passenger at the appropriate check-in area, where it is tagged and sent (generally by conveyor belt) to the baggage sortation room. Baggage arriving at the sortation room is sorted (either manually or mechanically) onto an appropriate bag cart, which is then transported to the aircraft where it is subsequently loaded into the cargo hull. Once again, if the departing aircraft is a wide-body, baggage is generally containerized at some point before it is loaded.

\section{Normal Transfer Baggage}

One sequence to describe the flow of normal transfer baggage follows the first two steps in the inbound baggage sequence and the last three steps of the outbound baggage sequence illustrated in Figure 7.1. Transfer baggage is unloaded (along with inbound baggage) from the aircraft and transported to the sortation room where it is separated by destination and/or departing flight numbers. The transfer baggage is then put into bag carts (along with outbound baggage) and transported to the departing aircraft where it 
is again loaded into the cargo hull. Note that even if the arrival and departure gates are adjacent, normal transfer baggage is generally taken back to the sortation room before it is transported to the departing aircraft.

\section{Ramp Transfer Baggage}

Also known as short connection baggage, ramp transfer baggage is taken directly from the arriving aircraft to the departing aircraft(s), often by a special baggage (or ramp) truck. The most common way for baggage to become designated as "short connection" baggage is through flight delays. A late arrival into a station can cause delays for downline connecting flights that are frequently held until passengers and baggage can be transferred. Passengers can often sprint from their arrival gate to their departure gate, particularly if it is within the same concourse. Normal transfer baggage, however, would follow the sequence described in the previous section, where it would be unloaded from the aircraft, transported to the sortation room, and then transported back to the departing aircraft.

To save time, short connection baggage is loaded directly onto ramp trucks, transported and loaded onto aircraft (or first into containers). This process adds to the cost of baggage handling operations, but reduces the (potentially higher) costs associated with the propagation of delays throughout the daily flight schedule. Ramp transfer baggage can also be "created" during the flight scheduling process. If a close connection is advantageous to an airline for competitive reasons, it may be willing to incur the marginal costs associated with operating additional ramp transfer trucks. 


\section{2 - Estimating Expected Transfer Times for Baggage}

If we condense the columns in Figure 7.1 to include only categories of like operations, we see that the handling process for all four types of baggage consists of three basic components; namely, loading/unloading, sortation, and transport/conveyance operations. Thus, the expected baggage transfer time will be a function of these three components. We define $w_{i j}$ as the time it takes for baggage to be transferred between point $i$ and point $j$ within the airport, or

$$
w_{i j}=f(L, S, C)
$$

where

$$
\begin{aligned}
& \mathrm{L}=\text { time associated with related loading and unloading operations } \\
& \mathrm{S}=\text { time associated with related sorting operations } \\
& \mathrm{C}=\text { time associated with related conveyance and transport operations }
\end{aligned}
$$

The next three sections discuss issues related to estimating each of these components.

\subsection{1 - Loading and Unloading Times}

Estimating the time associated with loading and unloading operations for a given flight can be accomplished either directly or indirectly. Under the direct method, explicit motion-time studies are performed on each element of the loading (unloading) operation, and based upon standards set for such operations an overall time can be estimated (for a single person) by multiplying the standard and the estimated number of bags for the flight. For example, one possible sequence for loading baggage into a small aircraft (i.e. no containers) is given below :

\section{Description of Operation}

Take bags off truck Place bags on conveyor

Conveyance time

Put bags into cargo hull

Total Loading Time (1 Bag)

\author{
$\underline{\text { Standard Time }}$ \\ $2 \mathrm{sec}$. \\ $4 \mathrm{sec}$. \\ $8 \mathrm{sec}$. \\ $6 \mathrm{sec}$.
}

20 sec. 
Also, let us assume the aircraft being loaded has a capacity of 128 seats, an average load factor of 67 percent, and the average number of bags carried per person is 1.3 .

Once the conveyor is full, the operation is limited only by the first two and last operations, which would typically be performed by two separate operators. Thus the loading time for multiple bags (once the conveyor is full) is only slightly larger than 6 seconds per bag (say 6.5 seconds) for two operators. One possible total loading time estimate, $L$, for this flight might be

$$
L=\frac{(128) *(.67) *(1.3) *(6.5)}{60}=12.1 \mathrm{~min}
$$

The difficulty with the direct method in a planning context is that many of the above factors are unknown or at least highly uncertain. In the absence of a detailed flight schedule, we are again confronted with a situation where it is necessary to make (perhaps incorrect) assumptions about aircraft mix and the sophistication of baggage handling equipment. In such cases, it is likely that the indirect method of establishing loading and unloading times would be more appropriate.

Under the indirect method, a standard or "target norm" is set for loading and unloading operations, and it is assumed that manpower requirements will be determined such that this standard is achieved with some given reliability. When discussing the time to unload bags from an aircraft, Ashford et al. [1991] note that :

considerable variation in unloading time can be encountered even with identical aircraft with similar loads, and the size of the aircraft is not necessarily a good indication of speed of unloading. Typically a performance standard is set, such as percentage of flights failing to meet 20 minutes delivery time for first bag and 35 minutes for last bag. [p. 199]

Thus, when trying to estimate the $L$ component of $\mathrm{w}_{\mathrm{ij}}$ in Equation 7.1, the simplest and perhaps most appropriate method to use might be the indirect method. This method is particularly useful if very different aircraft mixes are likely, where the direct method would require an additional analysis of making up and undoing containerized baggage. 


\subsection{2 - Sortation Times}

The time associated with sorting baggage (for a particular flight), $S$, depends on several interdependent factors, including: the level of automation of the baggage handling system, the size of the flight (in terms of expected number of bags), and the number and size of other flights. In this section we discuss how these factors might influence an estimate for $S$ in Equation 7.1.

The level of automation in place at an outbound bag room or baggage make-up area can be described as manual, semiautomated, or fully automated [Ashford et al. 1991]. Manual systems are the simplest. Bags are conveyed from the check-in area to the bag room where their destination or flight tags are read by human operators and sorted onto the appropriate bag carts. In semiautomated systems, flight tags on incoming baggage are also read by human operators who punch the tag code into a computer. A pulley arm is then activated which diverts the bag from the central conveyor to a human operator who places the bag onto the appropriate bag cart. Fully automated systems use laser readers and bar-coded bag tags to replace the human operator in the first step of the sortation process. Differences in sortation times (based on the level of automation) arise from differences in the time it takes to identify and divert the bags to the appropriate bag cart.

In addition to the level of automation present, the total sortation time also naturally depends on the actual number of bags expected for that particular flight. Once a bag is diverted from the main conveyor to the appropriate bag cart station, the bags must be taken off the conveyor and placed onto the bag cart. For large aircraft, bags must also be placed into containers. Direct calculations can be used to determine the expected time for making up the bag cart based on established methods, expected aircraft size and average load factors, or indirect assumptions can be made by establishing time standards.

Unlike loading and unloading operations, the number and size of other flights at the airport will also influence the estimate for the total sortation time for a given flight. Specifically, the number of other flights will influence the time it takes for bags to be identified and diverted from the main conveyor. The arrival rate of bags entering the sortation process depends on the arrival rate of passengers and can be determined from a cumulative arrival curve, such as the one illustrated in Figure 7.2. Based on the 
number of "concurrent" flights and the cumulative arrival rates for each, it is possible to estimate how long the sortation process might take, or at least whether the arrival process of passengers would be the dominant factor in the estimate for $S$ in Equation 7.1.

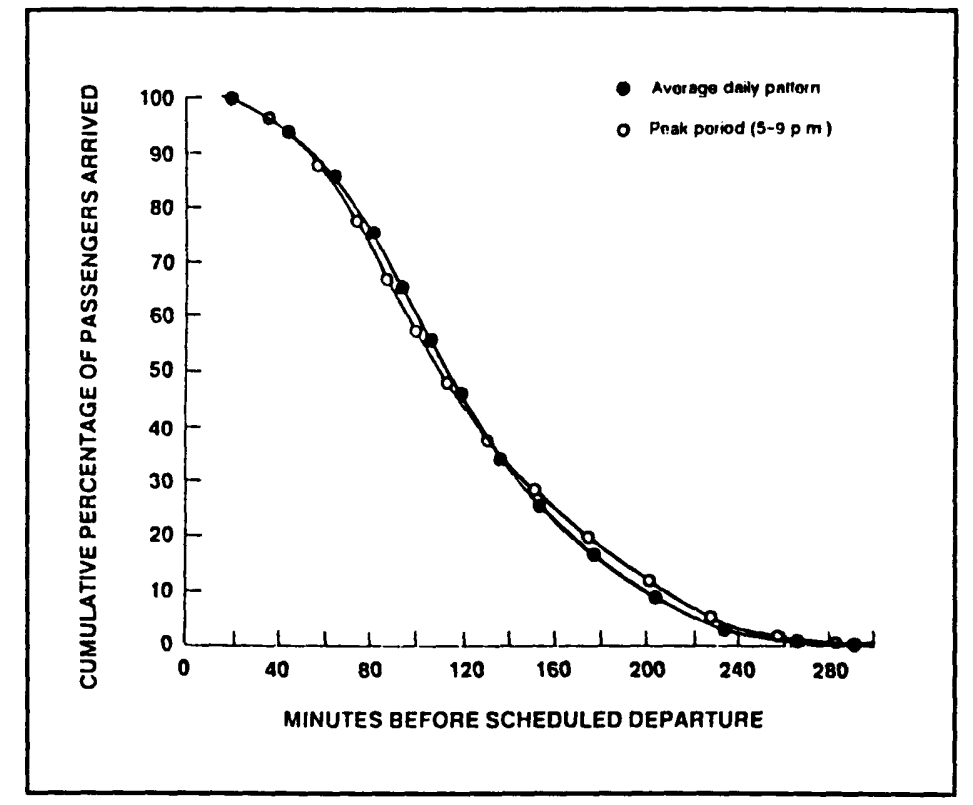

Figure 7.2: Examples of Cumulative Arrival Curves ${ }^{3}$

\subsection{3 - Conveyance and Transport Times}

Of the three basic components $(L, S, C)$ listed in Equation 7.1, the conveyance and transport time component of $w_{i j}$ is most directly influenced by the geometric configuration of the passenger buildings. Similar to passengers, baggage transiting from point $i$ to point $j$ will generally follow a specific path, which can be determined from a geometric representation of the airport layout (discussed in Chapter 4). By overlaying a Cartesian grid on the layout, the length of each path can be obtained.

Note that the path for bags going from $i$ to $j$ may be quite different than the path followed by passengers. Normal transfer bags arriving and departing from adjacent gates generally return to the sortation room before being loaded onto the appropriate departing aircraft, whereas direct transfer

${ }^{3}$ Source : [Transportation Research Board 1987], p. 96. 
passengers can simply walk to their departure gate. Conversely, ramp transfer trucks have the freedom to travel across apron areas (depending on aircraft congestion), whereas passengers transferring between buildings are confined to the boundaries of the landside configuration.

Nevertheless, if it is possible to identify the specific paths generally followed by baggage to and from all points within the airport, it is also possible to estimate the time it would take to traverse these paths. Similar to Equation 5.16, we can express the total conveyance and transport time for baggage transiting between points $i$ and $j, c_{i j}$, as the sum of the times spent on each mode $m$ along the (baggage) path from $i$ to $j, \mathrm{P}_{\mathrm{ij}}$, or

$$
c_{i j}=\sum_{m} c_{m} \quad \forall m \in P_{i j}
$$

Again, estimates for the values of $c_{m}$ will depend on the particular mode used (e.g. conveyor, bag cart, ramp transfer truck, etc.).

\subsection{4 - General Model for Expected Transfer Time}

One model that can be used to estimate the overall expected transfer time for baggage is similar to the one used to estimate the overall expected walking distance for passengers (Chapter 5). For a set of baggage categories $i(=1,2,3, \ldots n)$, the overall expected transfer time, $\bar{W}$, can be expressed as

$$
\bar{W}=\sum_{i=1}^{n} p^{i} \bar{w}^{i}
$$

where

$\bar{W} \quad=\quad$ overall average transfer time for baggage

$p^{i}=$ proportion of baggage belonging to category $i$

$\bar{w}^{i}=$ transfer time estimate for baggage category $i$ 
Each of the intermediate transfer time estimates, $\bar{w}^{i}$, is in turn a weighted average of the individual transfer time estimates, $\mathrm{w}_{\mathrm{ij}}$ (Equation 7.1), weighted by the probability that baggage will follow that path. For example, the expected transfer time for outbound baggage can be expressed as

$$
\bar{w}^{o b}=\sum_{j=1}^{G} w_{e j}^{o b} p_{e j}^{o b}
$$

where

$$
\begin{array}{lll}
\bar{w}^{o b} & = & \text { expected transfer time for outbound baggage } \\
w_{e j}^{o b} & = & \text { estimate for transfer time between entrance } e \text { and gate } j \\
p_{e j}^{o b} & = & \text { probability of baggage traveling between entrance } e \text { and gate } j \\
\mathrm{G} & = & \text { total number of gates at the airport }
\end{array}
$$

Equations for inbound, normal and ramp transfer baggage can also be found by analogy to Equations 5.2 and 5.4.

\subsection{5 - Estimating Baggage Transition Probabilities}

The values for $p_{e j}^{o b}$ in Equation 7.5 represent the probability that outbound baggage will follow a path from entrance $e$ of the airport to a specified departure gate $j$. This probability is, in some sense, equivalent to the demand share of Gate $j$ as discussed in Section 5.2.2. In the absence of any specific data regarding the distribution of passengers within the airport, we might employ a uniform assumption which assumes the probability that a passenger (and therefore his or her baggage) will depart from any gate within the airport is simply $1 / G$. If more information is available, such as the gate management policies in place and/or the mix of aircraft expected at individual gates, then these probabilities can be altered using an expression similar to Equation 5.12. 
In general, we can use the transition probabilities for originating and terminating passengers (from Chapter 5) to represent the transition probabilities for outbound and inbound baggage. Such an assumption is valid only if we also assume that the number of bags carried per passenger does not depend on the departure gate. For example, if gates located close to the check-in area were used exclusively for shuttle-type flights (typically passengers on such flights carry their bags onboard), then the transition probabilities used for passengers would not be the same as those for baggage. Under such circumstances, a separate analysis would have to be performed which took into consideration the expected number of bags per passenger for originating and terminating traffic at each individual gate.

We can also use the transition probabilities estimated for transfer passengers in the model for estimating the expected transfer times for normal and ramp transfer baggage. In this case, however, the analogy between passengers and baggage is not as strong. Normal transfer baggage might be assumed to follow equivalent paths to direct (or intraline) transfer passengers. In other words, if passengers are more likely to transfer between adjacent gates, so will their bags. As mentioned previously, the actual paths that passengers will follow are likely to be shorter, but the arrival and departure points ( $i$ and $j$ ) will be the same.

Ramp transfer baggage does not necessarily follow the same paths as indirect (or interline) passengers, and is a function of the daily operations and scheduling procedures of the individual airlines. In such cases, it might be appropriate to assume only that arriving ramp transfer baggage is more likely to depart from gates operated by the same airline, and that short connections may occur more frequently within the same concourse (as airlines tend to operate their hubs at a "cluster" of gates when possible).

In summary, it is possible to use the techniques introduced in Chapter 5 to estimate the overall expected transfer time for baggage using similarly structured data. Indeed, some of the specific passenger . related data can even be used, depending on the assumptions made regarding the distribution of baggage (versus the distribution of passengers) within the airport. 


\section{3 - Aircraft Taxi Distances and Times}

Similar to issues related to baggage handling, issues related to aircraft taxi distances and times can also be studied using data structured similarly to those for passenger walking distances. Airlines in particular are concerned about aircraft taxi times, because any delay will increase costs (crew, fuel, etc.) and may decrease the utilization. Therefore, it is useful during the early planning stages to examine how the geometry of a particular configuration will affect aircraft taxi distances and therefore taxi times.

Indeed, if we can model taxi times as we have passenger walking distances and baggage transfer times, we can include specific statistics in the decision making process of selecting an initial configuration. This section describes one possible model that could be used to estimate two of the primary inputs used to calculate aircraft taxi times; namely, absolute taxi distances and the required number of turns.

\subsection{1 - Factors Influencing Aircraft Taxi Times}

There are three primary components of the taxiing operation which influence the amount of time it takes for an aircraft to travel from the runway to the appropriate arrival gate. These components are

- Apron Congestion

- Absolute Distance

- $\quad$ Number of Aircraft Turns

Therefore, similar to the expected baggage transfer time, we can define the expected aircraft taxi time between exit taxiway $i$ and arrival gate $j, \mathbf{k}_{\mathrm{ij}}$, as

$$
k_{i j}=f(A, D, T)
$$

where
$A=$ Waiting time associated with apron congestion
$\mathrm{D}=$ Absolute distance of path between points $i$ and $j$
$\mathrm{T}=$ Number of aircraft turns required to travel between points $i$ and $j$ 
The waiting time associated with the amount of congestion on the apron is a function of the daily schedule (including irregular operations and delays) and the flight-to-gate assignments in place for incoming aircraft. As mentioned previously, it is very difficult to estimate such components during the planning stages given the absence of a detailed flight schedule and the amount of uncertainty associated with forecasts for the number of operations during a given period of time. Time and schedule-dependent issues such as apron congestion are currently handled by detailed, Monte Carlo simulation programs which include, as part of their input data, a detailed flight schedule.

\subsection{2 - Absolute Distance of Path}

The actual distance traveled by a taxiing aircraft naturally depends on the location of the starting and ending points of the path. The starting point, $i$, is assumed to be the beginning of the exit taxiway or turnoff from the landing runway. According to Horonjeff and McKelvey [1983],

The function of the exit taxiway, or turnoffs as they are sometimes called, is to minimize runway occupancy by landing aircraft ... The location of exit taxiways depends a great deal on the mix of aircraft, the approach and touchdown speeds, the point of touchdown, the exit speed, the rate of deceleration, which in turn depends on the condition of the pavement surface (e.g. dry or wet), and the number of exits. [p. 302,305]

Mathematical models have been developed to determine the optimal locations of exit taxiways [Horonjeff 1959], though more simplified methods can be found in reference manuals and texts [FAA 1980, 1982]. We assume that the locations of exit taxiways are given, and thus can be identified by an ordered pair on a Cartesian grid. Likewise, for a given configuration we assume that the location of the arrival gate, $j$, can also be identified by an ordered pair. In the absence of barriers, the distance of the path from $i$ to $j, \mathrm{~d}_{\mathrm{ij}}$, could therefore be found using Equation 5.3. Two potential barriers to a direct path typically exist, however, namely the amount of adequately paved apron space, and the configuration of the passenger buildings. 


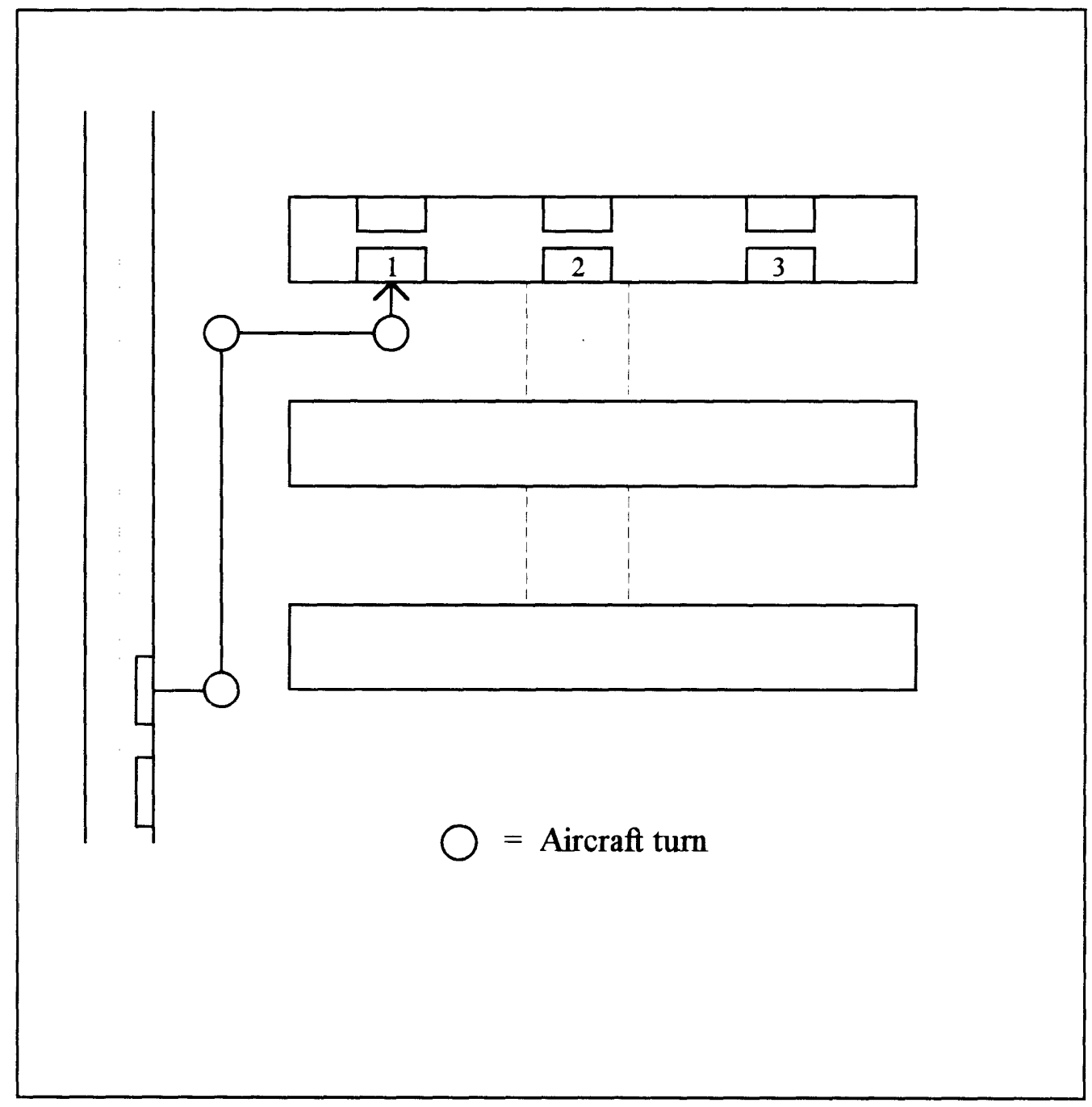

Figure 7.3 : Possible Aircraft Taxi Path to Gate 1 


\section{Configuration of Passenger Buildings}

The paths that aircraft can follow from the exit taxiway to the arrival gate are limited to those areas that are adequately paved to support the loads of the expected aircraft. In addition, the configuration of the airport passenger buildings will influence the paths taken by aircraft. Specifically, the geometric design of the passenger buildings (e.g. "X" or "T"-shaped, circular, rectangular) can produce natural barriers to the most direct path, and the presence of above grade connectors can produce "cul-de-sacs" which can be accessed only from a single location. Both of these barriers will tend to increase the length of the most direct path from the exit taxiway to the arrival gate, even if the entire apron area is adequately paved.

Thus, when determining the actual distances traveled by aircraft, $d_{i j}$, it is necessary to consider not only the physical locations of the beginning and end points, but also the existence of barriers. Most notably, barriers such as the structural design of the pavement and the specific configuration of the passenger buildings (including connectors between buildings) should be considered.

\subsection{3 - Number of Aircraft Turns}

The second factor influencing aircraft taxi times is the number of turns required to complete the path from exit taxiway $i$ to arrival gate $j$. As mentioned in the previous section, the structural design of the pavement and the configuration of the passenger buildings both can increase the length of the most direct path from $i$ to $j$ by producing barriers. In general, aircraft avoid these barriers by following more circuitous paths, which may include a number of turns.

The primary effect of a required turn is to slow the aircraft down, decreasing its average speed. Thus, in addition to absolute path distance, it may be desirable to maintain a turn matrix, $T$, whose members, $\mathrm{t}_{\mathrm{ij}}$, represent the number of aircraft turns required along the path from $i$ to $j$. When comparing different configurations based on taxi distances, the expected number of turns could be used for additional information, to "break ties" for example. 


\section{4 - Estimating Transition Probabilities}

Once matrices have been obtained for the actual distances traveled and the number of turns required for arriving aircraft, it is then necessary to determine the matrix of transition probabilities, $P$, similar to those defined in Chapter 5 . In this case, each element $\mathbf{p}_{\mathrm{ij}}$ represents the unconditional probability that an aircraft will exit from taxiway $i$ and proceed to arrival gate $j$. The general expression for the expected taxi distance, $\bar{D}$, is again simply

$$
\bar{D}=\sum_{i=1}^{E} \sum_{j=1}^{G} p_{i j} d_{i j}
$$

where

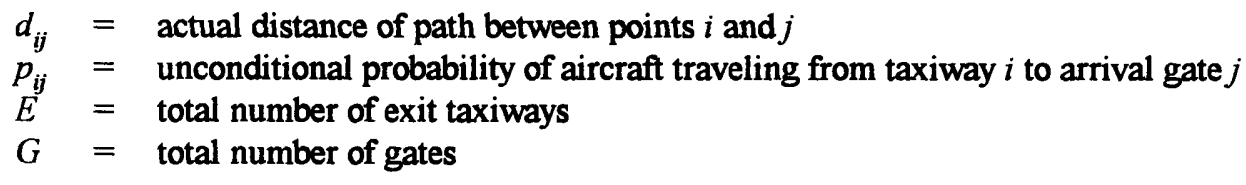

Alternatively, we can use conditional probabilities to express $p_{i j}$, in which case the above expression becomes

$$
\bar{D}=\sum_{i=1}^{E} \sum_{j=1}^{G} p_{i} p_{j / i} d_{i j}
$$

where

$$
\begin{aligned}
p_{i}= & \text { unconditional probability aircraft will exit from taxiway } i \\
p_{j / i}= & \text { conditional probability of aircraft traveling to arrival gate } j, \\
& \text { given it has exited from taxiway } i
\end{aligned}
$$

Equivalent expressions can be obtained, by analogy, to estimate the expected number of turns, $\bar{T}$. 


\subsection{1 - Unconditional Exit Taxiway Probabilities}

This section presents one possible model for estimating the unconditional probability of an aircraft exiting from taxiway $i$. The complexity of the model depends on the amount of information available to the planner. In the absence of any other information, we could employ a uniform probability assumption such that for a total of $E$ exit taxiways, the probability that an aircraft will exit from taxiway $i$ is simply $\frac{1}{E}$. Other factors exist, however, which influence the individual taxiway used. Specifically, we consider : the runway configuration being used, the size of the aircraft, and the condition of the pavement (i.e. dry or wet). Additionally, the location and airline presence at each passenger building may influence which taxiway is used. These factors can be included, by analogy, into the expressions derived in this section. There are omitted here for simplicity.

\section{Runway Configuration}

The particular runway configuration used depends largely on the current direction of the wind. Meteorological data is maintained, often in the form of a wind rose, from which it is possible to determine the directional frequency of the prevailing winds. Based on such data, we can estimate the probability that a given runway will be in use. We define this probability as $p_{r}\left(r_{0}\right)$, where $\mathrm{r}_{0}$ is one of $R$ total runways at the airport. Each runway is assumed to have $E_{r_{0}}$ exit taxiways, such that

$$
\sum_{r_{0}=1}^{R} E_{r_{0}}=E
$$

Each of the $E$ individual taxiways is also assumed to have its own unique number, $\mathrm{e}_{0}=\{1,2,3, \ldots E\}$. We define $p_{e / r}\left(e_{0} \mid r_{0}\right)$ as the conditional probability that an aircraft will exit from taxiway $e_{0}$ given that it has landed on runway $r_{0}$. If no other information is given regarding the use of the exit taxiways for a given 
runway $r_{0}$, we could again employ a uniform distribution assumption. This time, however, we apply the assumption only to the $E_{r_{0}}$ taxiways of runway $\mathrm{r}_{0}$. The expression for $\mathrm{p}_{\mathrm{i}}$ in Equation 7.8 becomes

$$
p_{i}=p_{r}\left(r_{0}\right) \cdot p_{e / r}\left(e_{0} \mid r_{0}\right)
$$

where

$$
p_{e / r}\left(e_{0} \mid r_{0}\right)= \begin{cases}\frac{1}{E_{r_{0}}} & \forall e_{0} \in r_{0} \\ 0 & \text { otherwise }\end{cases}
$$

Such a uniform assumption, however, still neglects the influence of aircraft size and runway pavement conditions, both of which are important factors in determining which exit taxiway will be used for a given runway.

\section{Aircraft Size}

In general, larger aircraft have longer stopping distances than smaller aircraft. Therefore, it may be possible to improve upon the uniform distribution assumption if we are given information regarding the mix and typical stopping distances required for the different types of aircraft expected. For example, we might employ different distributions to describe conditional probabilities $p_{e / r, a}\left(e_{0} \mid r_{0}, a_{0}\right)$, where the new conditioning event, $a$, represents the type of aircraft. Figure 7.4(a) illustrates three distributions, intended to demonstrate possible differences in exit taxiway use for three different aircraft types. In each case we assume the exit taxiways are numbered in order (from lowest to highest) of their position on the runway. 


\section{Pavement Conditions}

In addition to aircraft type, the condition of the pavement due to weather also influences the exit taxiway used by aircraft. Horonjeff and McKelvey [1983] note that :

Slush or standing water on the runway has an undesirable effect on aircraft operation. Slush is equivalent to wet snow. It has a slippery texture which makes braking extremely poor. Being a fluid, it is displaced by tires rolling through it, causing a significant retarding force ... Both water and slush result in a very poor coefficient of braking friction. When tires ride on the surface of the water or slush, the phenomenon is known as hydroplaning. When the tires hydroplane, the coefficient of friction is on the order of wet ice and the steering ability is completely lost. [p. 95,96]

Thus when pavement conditions are poor, stopping distances increase. Under such conditions, the conditional probabilities illustrated in Figure 7.4(a) will likely change to reflect a greater probability of using exit taxiways further along the runway. We might, therefore, add a third conditioning event, $w$, to represent the weather or pavement conditions (e.g. wet or dry). Figure 7.4(b) illustrates possible differences in the conditional distributions if we consider weather in addition to aircraft size. Our expression for $p_{i}$ in Equation 7.8 thus becomes

$$
p_{i}=p_{r}\left(r_{0}\right) \cdot p_{a}\left(a_{0}\right) \cdot p_{w}\left(w_{0}\right) \cdot p_{e / r, a, w}\left(e_{0} \mid r_{0}, a_{0}, w_{0}\right)
$$

where

$$
\begin{aligned}
& p_{i}=\text { unconditional probability aircraft will exit from taxiway } i \\
& p_{r}\left(r_{0}\right)=\text { probability aircraft will land on runway } \mathrm{r}_{0} \\
& p_{a}\left(a_{0}\right)=\text { probability aircraft is of type } \mathrm{a}_{0} \\
& p_{w}\left(w_{0}\right)=\text { probability pavement is in condition } \mathrm{w}_{0}
\end{aligned}
$$

and

$$
\begin{aligned}
p_{e / r, a, w}\left(e_{0} \mid r_{0}, a_{0}, w_{0}\right)= & \text { Conditional probability that aircraft will exit from taxiway } i, \\
& \text { given that it has landed on runway } \mathrm{r}_{0} \text {, it is of type } \mathrm{a}_{0}, \\
& \text { and the pavement is in condition } \mathrm{w}_{0}
\end{aligned}
$$

Other factors can be included, if such information is available, by adding conditioning events to the righthand side of Equation 7.12. 


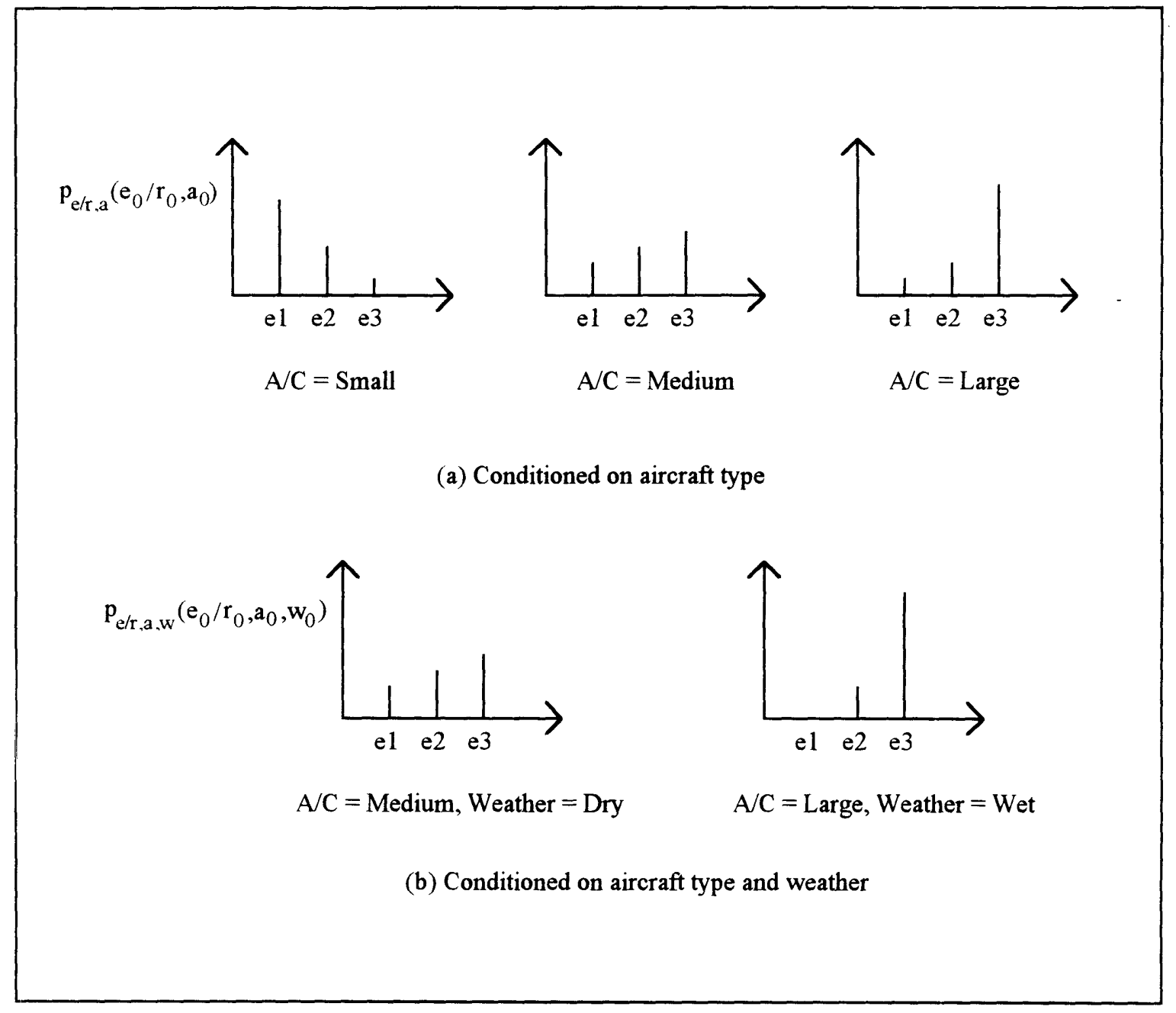

Figure 7.4: Conditional Probability Distributions 


\subsection{2 - Arrival Gate Probabilities}

Returning to Equation 7.8, we now consider how we might estimate the conditional probability of an aircraft taxiing to arrival gate $j$, given that it has exited from taxiway $i$. If no other information is given regarding gate utilization, we can again employ a uniform distribution assumption, such that for an airport with $G$ gates, the probability of arriving at gate $j$ is simply $1 / G$. As mentioned in Chapter 5 , however, such an assumption neglects the operational reality that different gates are used (perhaps preferentially) for different types of aircraft. Thus, the number of operations per day witnessed by each gate may be very different.

Based on information given for aircraft objects in Chapter 5 , it is possible to estimate the gate utilization, $u_{j}$, as measured by the number of operations performed over a given time period. Thus, the probability of an aircraft arriving at gate $j$ could be estimated by the share of total operations handled by gate $j$ during a given time period, or

$$
p_{j / i}=\frac{u_{j}}{\sum_{j=1}^{G} u_{j}}
$$

where

$$
\begin{array}{ll}
p_{j / i} \quad=\quad \text { Conditional probability aircraft will arrive at gate } j, \text { given it has } \\
\\
u_{\mathrm{j}} \quad=\quad \begin{array}{l}
\text { Opited from taxiway } i \\
\mathrm{G}
\end{array} \quad \text { Total number of gates }
\end{array}
$$

As mentioned previously, more sophisticated models can be developed based on the amount of information available to the planner, including the location and airline presence at individual concourses.

Once estimates for $p_{j / i}$ are obtained, it is possible to determine the estimates for the expected taxi distances and the expected number of aircraft turns for a given configuration. Based on this information, it is possible to estimate the overall expected taxi times if the relationship between turns and average speed is given. In the simplest case, we can use an expression similar to Equation 5.17 (used for 
estimating passenger travel times), where in this case the average speed of the aircraft is somehow decreased based on the required number of turns obtained from the previous analysis. Similar analyses can also be performed, by analogy, to determine departure taxi distances and turns.

\section{5 - Numerical Example}

We now demonstrate how an approach similar to the one used to estimate overall expected walking distances can be used to estimate overall aircraft taxi distances and the expected number of turns for different passenger building configurations. As an example, let us compare the relative performance of the two midfield concourse types described in Chapter 4; namely, the parallel linear and "X"-shaped concourses. Figure 7.5 illustrates one of the "X"-shaped concourses proposed for the new Kuala Lumpur International Airport. Note that unlike the new Pittsburgh Airport (which also has an "X"-shaped midfield concourse), the apron at Kuala Lumpur is not completely paved. Therefore, taxiing aircraft must follow specific (and sometimes circuitous) paths from exit taxiways to gates. From the figure, we can determine the approximate distances of these required paths to each of the aircraft stands located along the concourse perimeter.

For simplicity, let us assume that only two exit taxiways are used, denoted by Roman numerals I and II in the figure. Aircraft arriving at gates to the left of the centerline are assumed to exit from taxiway I, and those arriving at gates to the right are assumed to exit from taxiway II. Examples of two possible aircraft paths are illustrated by the dark lines in the figure. Each circle along the indicated paths denotes a required turn.

Using the appropriate scale, we obtain the distances from each exit taxiway to each gate, as well as the number of required turns for each specified path. These distance and turn data are summarized in Table 7.1. If we assume a single aircraft type and a uniform arrival pattern of aircraft to gates, the

expected taxi distance, $\bar{D}$, and the expected number of required turns, $\bar{R}$, can be obtained simply by 


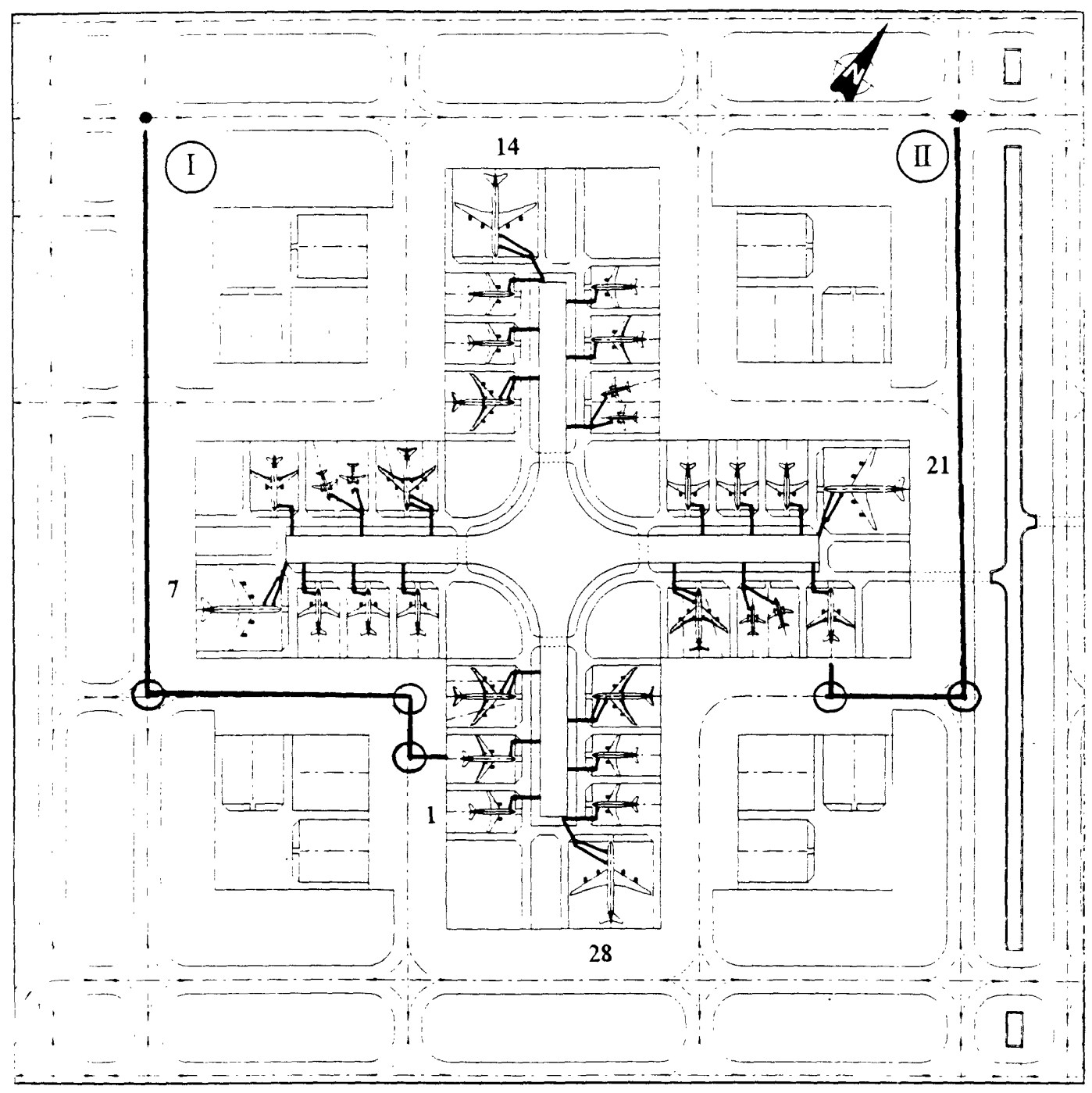

Figure 7.5 : "X"-Shaped Concourse for Proposed Kuala Lumpur International Airport ${ }^{4}$

${ }^{4}$ Source : [Anglo Japanese Consortium 1992], p. 97. 
taking the arithmetic means of the values given in Table 7.1, which yields

$$
\begin{aligned}
& \bar{D}=832.0 \mathrm{~m} \\
& \bar{R}=2.04 \text { turns } / \text { path }
\end{aligned}
$$

Table 7.1: Distance and Required Turn Data for "X"-Shaped Concourse

\begin{tabular}{cccccccc}
\hline $\begin{array}{c}\text { Gate } \\
\text { Number }\end{array}$ & $\begin{array}{c}\text { Exit } \\
\text { Taxiway }\end{array}$ & $\begin{array}{c}\text { Distance } \\
(\mathbf{m})\end{array}$ & $\begin{array}{c}\text { \# of } \\
\text { Turns }\end{array}$ & $\begin{array}{c}\text { Gate } \\
\text { Number }\end{array}$ & $\begin{array}{c}\text { Exit } \\
\text { Taxiway }\end{array}$ & $\begin{array}{c}\text { Distance } \\
(\mathbf{m})\end{array}$ & $\begin{array}{c}\text { \# of } \\
\text { Turns }\end{array}$ \\
\hline 1 & I & 1110 & 3 & 15 & II & 800 & 3 \\
2 & I & 1055 & 3 & 16 & II & 745 & 3 \\
3 & I & 990 & 1 & 17 & II & 690 & 1 \\
4 & I & 990 & 2 & 18 & II & 690 & 2 \\
5 & I & 950 & 2 & 19 & II & 650 & 2 \\
6 & I & 910 & 2 & 20 & II & 610 & 2 \\
7 & I & 650 & 1 & 21 & II & 530 & 1 \\
8 & I & 555 & 2 & 22 & II & 855 & 2 \\
9 & I & 620 & 2 & 23 & II & 920 & 2 \\
10 & I & 690 & 2 & 24 & II & 990 & 2 \\
11 & I & 690 & 1 & 25 & II & 990 & 1 \\
12 & I & 745 & 3 & 26 & II & 1055 & 3 \\
13 & I & 800 & 3 & 27 & II & 1110 & 3 \\
14 & I & 505 & 1 & 28 & II & 1400 & 2 \\
\hline
\end{tabular}

We could also assume, however, that two types of aircraft exist, Large and Small, making up 30 percent and 70 percent of the aircraft mix, respectively. If, say, Large aircraft can arrive only at Gates $7,14,21$, and 28 , the estimates for $\bar{D}$ and $\bar{R}$ become

$$
\begin{array}{lll}
\bar{D}=.30(771.25)+.70(831.96) & =813.7 \mathrm{~m} \\
\bar{R}=.30(1.25)+.70(2.04) & = & 1.80 \mathrm{turns} / \mathrm{path}
\end{array}
$$

Consider now the (hypothetical) parallel linear concourse illustrated in Figure 7.6 which contains the same number of aircraft stands as the "X"-shaped concourse in Figure 7.5. For consistency, we assume the taxiways parallel to the length of the linear concourse are located a distance equal to that from the taxiway to the closest edge of the "X"-shaped concourse (approximately $55 \mathrm{~m}$ ), and that the average gate spacing is the same (approximately $60 \mathrm{~m}$ ). For such a construction, the table of taxi distances and required number of turns is summarized in Table 7.2. 


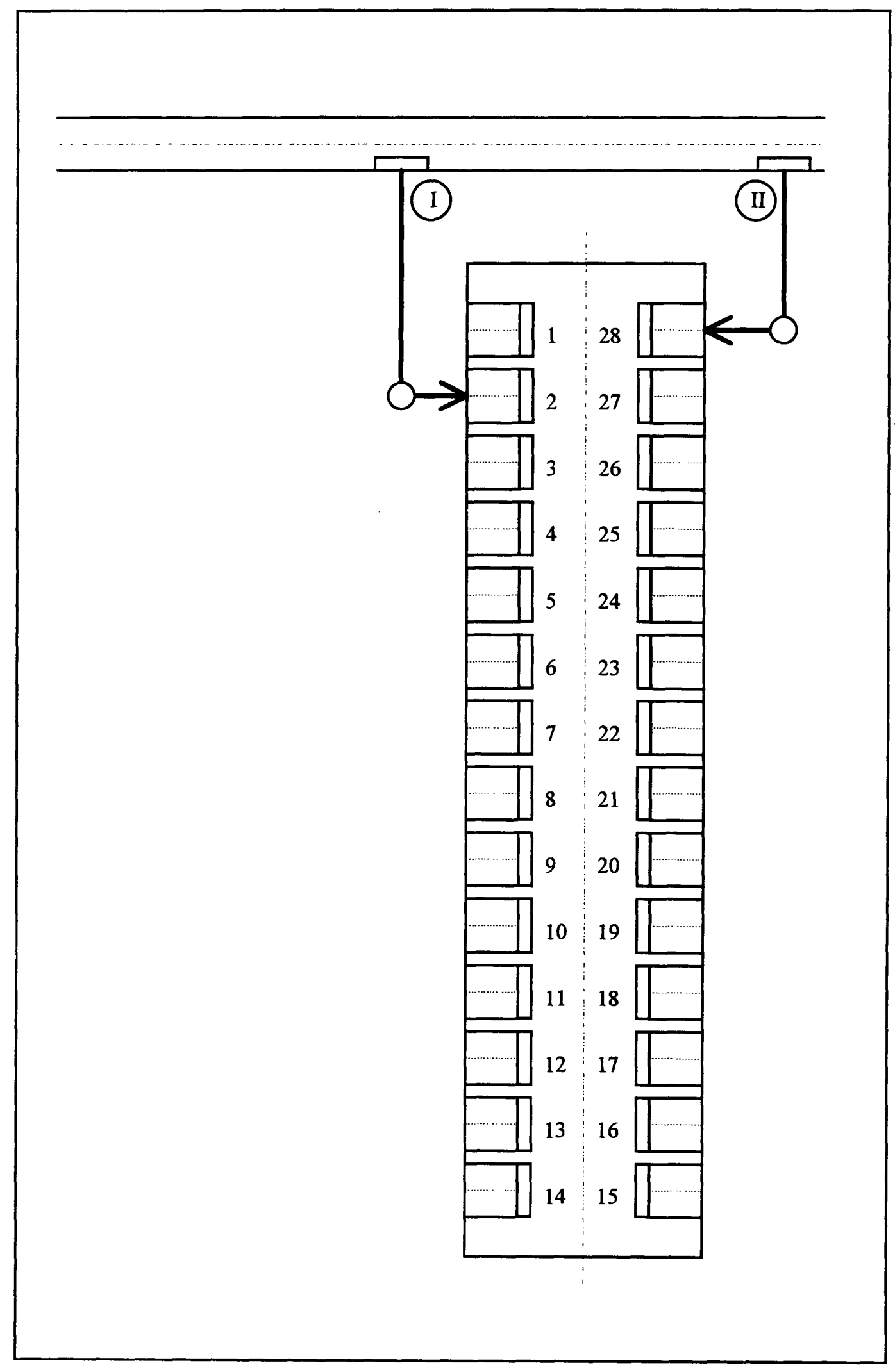

Figure 7.6: "Equivalent" Parallel Linear Concourse 
Table 7.2 : Distance and Required Turn Data for "Equivalent" Parallel Linear Concourse

\begin{tabular}{cccccccc}
\hline $\begin{array}{c}\text { Gate } \\
\text { Number }\end{array}$ & $\begin{array}{c}\text { Exit } \\
\text { Taxiway }\end{array}$ & $\begin{array}{c}\text { Distance } \\
(\mathrm{m})\end{array}$ & $\begin{array}{c}\text { \# of } \\
\text { Turns }\end{array}$ & $\begin{array}{c}\text { Gate } \\
\text { Number }\end{array}$ & $\begin{array}{c}\text { Exit } \\
\text { Taxiway }\end{array}$ & $\begin{array}{c}\text { Distance } \\
(\mathrm{m})\end{array}$ & $\begin{array}{c}\text { \# of } \\
\text { Turns }\end{array}$ \\
\hline & I & 200 & 1 & 15 & II & 980 & 1 \\
1 & I & 260 & 1 & 16 & II & 920 & 1 \\
2 & I & 320 & 1 & 17 & II & 860 & 1 \\
3 & I & 380 & 1 & 18 & II & 800 & 1 \\
4 & I & 440 & 1 & 19 & II & 740 & 1 \\
5 & I & 500 & 1 & 20 & II & 680 & 1 \\
6 & I & 560 & 1 & 21 & II & 620 & 1 \\
7 & I & 620 & 1 & 22 & II & 560 & 1 \\
8 & I & 680 & 1 & 23 & II & 500 & 1 \\
9 & I & 740 & 1 & 24 & II & 440 & 1 \\
10 & I & 800 & 1 & 25 & II & 380 & 1 \\
11 & I & 860 & 1 & 26 & II & 320 & 1 \\
12 & I & 920 & 1 & 27 & II & 260 & 1 \\
13 & I & 980 & 1 & 28 & II & 200 & 1 \\
14 & & & & & & & \\
\hline
\end{tabular}

Taking the simple arithmetic mean of the values listed in Table 7.2 yields

$$
\begin{aligned}
& \bar{D}=590.0 \mathrm{~m} \\
& \bar{R}=1.00 \mathrm{turns} / \text { path }
\end{aligned}
$$

The preceding example, though simplified, reinforces one of the central themes of this dissertation; namely, it is not enough to consider only a single criterion when evaluating the potential performance of a configuration. Based solely on the objective of minimizing overall expected walking distances, we have seen that the "X"-shaped concourse provides good performance under certain circumstances (Figure 6.17). This configuration, however, can increase expected taxi distances, and perhaps more importantly (due to an increase in the number of required turns), taxi times.

The parallel linear concourse increases the expected walking distances for passengers because it is longer in one dimension than the equivalent " $\mathrm{X}$ "-shaped concourse. There may exist geographical constraints, however, which limit the maximum concourse length in one direction. In this case, a configuration with four " $\mathrm{X}$ "-shaped concourses might be compared to one with six parallel linear 
concourses having the same maximum length (while keeping the total number of gates constant). Since the linear concourses can be positioned closer together (due to their shape), the latter configuration may actually require less "greenfield" acreage, making it more attractive in terms of real estate costs.

The selection of the most appropriate configuration concept must involve the evaluation of many different (often conflicting) criteria, and trade-offs between these criteria must be made during the decision making process. By developing a methodology for quickly evaluating many different measures of potential configuration performance, we approach the ultimate goal for the PBCE tools of providing airport planners with high speed, highly flexible decision support. In the next chapter, we present two more models for measuring potential configuration performance; namely, models for estimating passenger congestion along pedestrian walkways and congestion within waiting areas. 


\title{
Chapter 8
}

\section{Potential Congestion}

\author{
Introduction
}

Previous chapters have used similarly structured data to determine measures of potential configuration performance related to passenger convenience, baggage transfer times and aircraft taxi times. These measures are, in some sense, "static" metrics as they do not depend heavily on the time dependent nature of passenger and aircraft flow. Many issues related to passenger convenience depend on the dynamic nature of passenger flows, however. Waiting times in queues, for example, depend on the arrival rate of passengers at a service facility (e.g. check-in counters, security checkpoints) as well as the service rate of that facility. Even in areas where passengers do not congregate, such as walkways and stairways, pedestrian traffic flows can influence the convenience or comfort afforded passengers due to congestion.

Chapter 8 develops models to estimate potential congestion within specified areas of an airport passenger building. Section 8.1 introduces the concept of screen lines and demonstrates how they can be used in conjunction with data from previous chapters to identify potential congestion across a linear section of a building. Section 8.2 extends the work in Section 8.1 and applies specific level of service design standards to determine the required width of walkways and stairways. Section 8.3 reviews the 
IATA level of service design standards for areas, and presents a general model that can be used to determine spatial requirements for dwelling passengers. Finally, Section 8.4 presents a case study of Terminal E at Boston Logan International Airport, and applies some of the congestion models developed in previous sections as a proof of concept.

\section{1 - Screen Lines}

One means of measuring the potential congestion within an airport passenger building is to estimate the probability that a randomly selected passenger will cross a particular point in that building within a given period of time. Screen lines divide a passenger building into two parts and are oriented perpendicularly to the primary flow of traffic (Figure 8.1). On a Cartesian grid, they can be designated by an $\mathrm{x}$ - or a y-coordinate. We define $k$ as the direction of traffic under consideration, as illustrated in Figure 8.1(c). In Figure 8.1(a), for example, originating passengers departing from Gate 3 travel in direction $k=2$ from the entrance, and terminating passengers arriving at Gate 4 travel on direction $k=4$ toward the exit. Further, we define a variable $s_{\mathrm{ijk}}$, such that

$$
s_{i j k}=\left\{\begin{array}{l}
p_{i j} \text { if path from } i \text { to } j \text { crosses screen line in direction } k \\
0 \text { otherwise }
\end{array}\right.
$$

where

$$
\begin{aligned}
& p_{i j}=\text { the unconditional probability that a randomly selected passenger } \\
& \text { will follow along a path from point } i \text { to point } j \text { (as described in Chapter 5) }
\end{aligned}
$$

and

$$
\sum_{i} \sum_{j} p_{i j}=1
$$


The conditions under which a path "crosses" a screen line depend on the passenger category considered. In Figure 8.1(b), for example, transfer passengers arriving at Gate 1 and departing from Gate 3 will cross the given screen line in direction $k=1$, whereas terminating passengers arriving at Gate 1 will not.

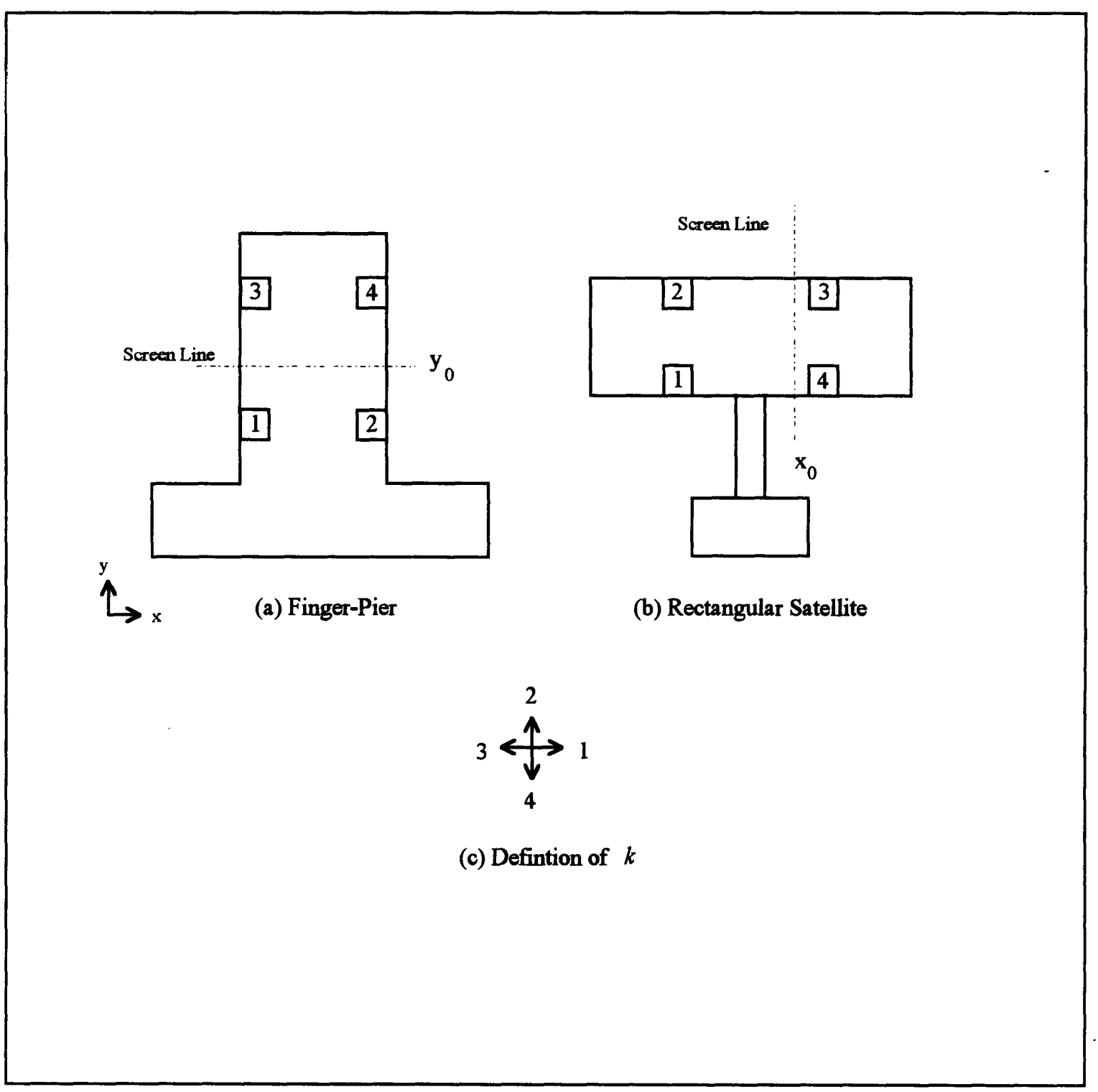

Figure 8.1 : Examples of Screen Lines 
By summing $s_{\mathrm{ijk}}$ over all possible paths (for a given direction), we can obtain one measure of the potential congestion across the screen line. We define a function $S_{k}\left(y_{0}\right)$ to denote the probability that a passenger will cross a screen line located at $\mathrm{y}_{0}$ (in a single direction $k$ ), or

$$
S_{k}\left(y_{0}\right)=\sum_{\text {All paths }} s_{i j k}
$$

We can use multiple subscripts for $k$ in Equation 8.3 to denote potential congestion in more than one direction and similar expressions can be used for screen lines oriented perpendicularly to the $\mathrm{x}$-axis, or $S_{k}\left(x_{0}\right)$.

\section{Congestion Profiles}

Note that since all paths begin and end at discrete locations (such as gates and entrances), the value of $S_{k}\left(y_{0}\right)$ changes only at y values associated with those locations. We can express the values of $S_{k}\left(y_{0}\right)$ graphically as a "congestion profile", which can be used to identify areas of potential congestion. Figure 8.2(a) illustrates a congestion profile in the form of a step function for the finger-pier concourse shown in Figure 8.1(a). We can also represent the same information by assigning a gray scale value to each different function value (i.e. the higher the value of $S_{k}\left(y_{0}\right)$, the darker the shade of gray), and shade in the actual layout. Such a representation is shown for the same finger-pier concourse in Figure 8.2(b).

Note that beyond Gates 3 and 4 (in direction $k=2$ ) the congestion profile is white, suggesting that there is no congestion beyond a screen line located there. In a true operational setting, passengers waiting to depart from these gates are likely to congregate in this area, particularly if concession stands are located there. The profile is meant to express congestion for required paths, rather than to anticipate passenger behaviors. 


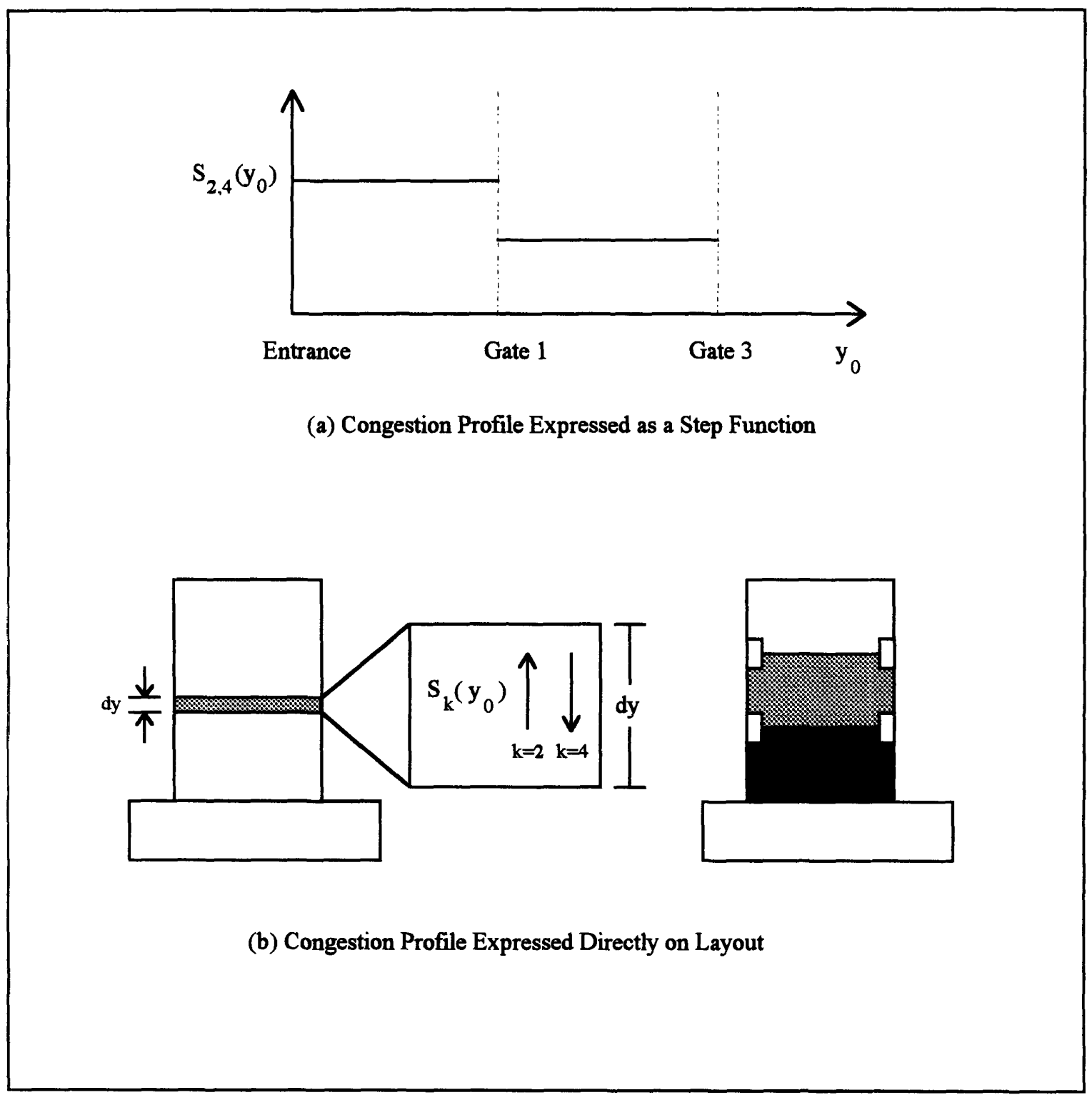

Figure 8.2 : Different Representations of a Congestion Profile 


\section{2 - Congestion Across Screen Lines}

As mentioned previously, the function $S_{k}\left(y_{0}\right)$ represents the probability that a randomly selected passenger will cross a screen line (located a $y_{0}$ ) within a given time interval. If we subdivide the timeaxis into periods $\mathrm{t}=\{1,2, \ldots, n\}$, then $S_{k}\left(y_{0}\right)$ represents the fraction of total traffic that is expected to cross the screen line within some time interval, $t$. If the data used to calculate $S_{k}\left(y_{0}\right)$ are sufficiently time-specific, then it is possible to estimate the total number of passengers, $N(t)$, expected to cross a screen line at $y_{0}$, by multiplying $S_{k}\left(y_{0}\right)$ by the expected volume of traffic, $V(t)$, at the airport for the time interval $t$, or simply

$$
N(t)=V(t) \cdot S_{k}\left(y_{0}\right)
$$

where

$$
\begin{aligned}
& N(t)=\text { expected number of passengers to cross screen line during time } t \\
& V(t) \quad=\quad \text { total traffic volume expected at airport during time } t \\
& S_{k}\left(y_{0}\right)=\text { unconditional probability that a randomly selected passenger } \\
& \text { will cross screen line located at } y_{0}
\end{aligned}
$$

Typical planning level forecasts for passenger traffic include a peak hour statistic, which is expressed in a variety of ways [Ashford et al. 1991], including :

- a percentage of annual passenger flows

- the thirtieth highest hour of passenger flow

- the hourly rate above which 5 percent of the traffic of the airport is handled

This peak hour level is used for designing spaces within passenger buildings in order to handle adequately the expected passenger volumes during all but a small fraction of the busiest time periods. 


\section{Designing for Passenger Flows}

Fruin [1971] suggests that the level of convenience (in terms of congestion) afforded pedestrians

(such as passengers within an airport building) can be expressed in terms of a statistic he calls :

the flow volume $(\mathrm{P})$-- the number of traffic units passing a point per unit time. In pedestrian design, flow is expressed as pedestrians per foot width of the walkway or stairway per minute (PFM). Flow is the most important traffic statistic, because it determines the width of the pedestrian way (pedway). An inadequate width restricts flow, resulting in passenger inconvenience. [p. 37]

Although average pedestrian walking speeds have been shown to vary due to such factors as time of day, trip purpose, and grade (above six percent), the most significant determinant of walking speed is traffic density [Fruin 1971]. Pedestrian speeds decrease as traffic density increases due to a reduction in the available clear area for movement (i.e. crowding occurs). Time-lapse photography studies have been used to establish flow-volume relationships for various categories of pedestrian Traffic (Figure 8.3). According to Fruin,

these relationships, representing the average conditions of three distinctive types of pedestrian traffic, show a relatively small range of variation, which strongly suggests that reverse and cross-flow traffic conflicts do not drastically reduce either pedestrian traffic volume or speed. This characteristic makes these curves applicable to a wider range of different design conditions. [p. 43]

Level of service design standards have been established for different passenger flow volumes and are typically expressed in terms of average pedestrian area occupancy ( $\mathrm{ft}^{2} /$ person), and average flow volumes (PFM). These standards vary according to the types of passenger flow considered. Table 8.1 lists standards for two flow types important to the design of airport passenger buildings; namely, walkways and . stairways. The following sections illustrate how to combine the function $S_{k}\left(y_{0}\right)$, the peak hour forecast and the different level of service design standards in order to determine the required width of walkways and stairways within airport passenger buildings. 


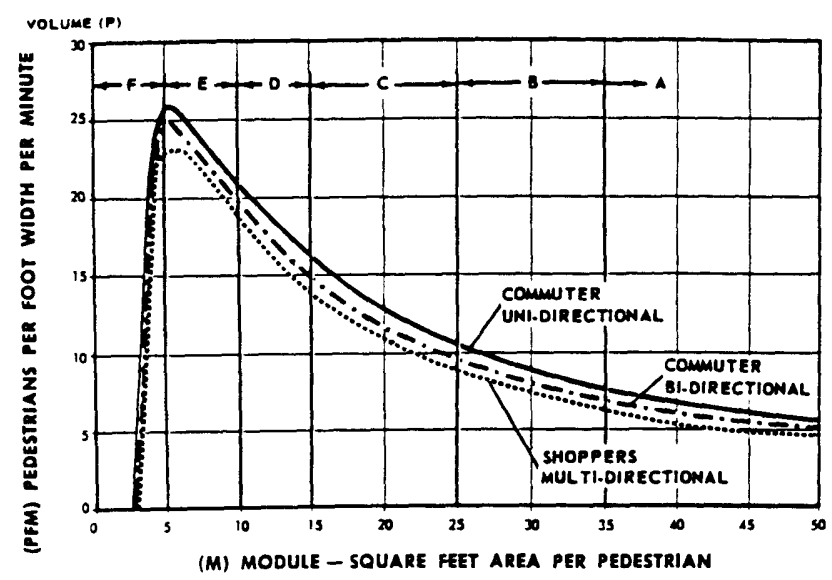

(a) Level of Service Standards for Walkways

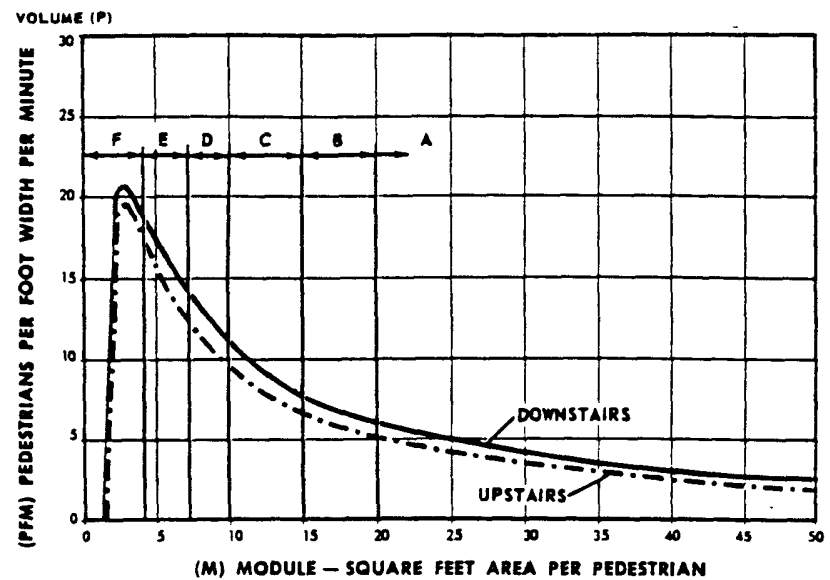

(b) Level of Service Standards for Stairways

Figure 8.3 : Flow-Volume Relationships for Pedways ${ }^{1}$

\footnotetext{
${ }^{1}$ Source: [Fruin 1971], p. 78,84.
} 
Table 8.1 : Level of Service Design Standards for Walkways and Stairways ${ }^{2}$

\begin{tabular}{ccccc}
\hline \multicolumn{2}{c}{ Walkways } & \multicolumn{2}{c}{ Stairways } \\
\hline $\begin{array}{c}\text { Level } \\
\text { of } \\
\text { Service }\end{array}$ & $\begin{array}{c}\text { Avg. Pedestrian } \\
\text { Area Occupancy } \\
\left(\mathrm{ft}^{2} / \text { person) }\right.\end{array}$ & $\begin{array}{c}\text { Avg. Flow } \\
\text { Volume }\end{array}$ & $\begin{array}{c}\text { Avg. Pedestrian } \\
\text { Area Occupancy } \\
\left(\mathrm{ft}^{2} / \text { person }\right)\end{array}$ & $\begin{array}{c}\text { Avg. Flow } \\
\text { Volume } \\
(\text { PFM })\end{array}$ \\
\hline & & & & \\
\hline A & $>35$ & $<-10$ & & $<5$ \\
B & $25-35$ & $7-10$ & $15-20$ & $5-7$ \\
C & $15-25$ & $10-15$ & $10-15$ & $7-10$ \\
D & $10-15$ & $15-20$ & $7-10$ & $10-13$ \\
E & $5-10$ & $20-25$ & $4-7$ & $13-17$ \\
F & $<5$ & $>25$ & $<4$ & $>17$ \\
\hline
\end{tabular}

\subsection{1 - General Model}

From Equation 8.1 we can obtain the expected number of passengers to cross a screen line located at $\mathrm{y}_{0}\left(\mathrm{x}_{0}\right)$ during the peak hour. If we assume that traffic flow is roughly constant during that hour, then we can express the average flow volume, $P$, as

$$
P=\frac{S_{k}\left(y_{0}\right) \cdot T P H P}{60 W}
$$

where

$$
\begin{array}{ll}
P & =\text { average flow volume in passengers per foot width per minute } \\
S_{k}\left(y_{0}\right) & =\text { unconditional probability that a randomly selected passenger } \\
& \text { will cross screen line located at } y_{0} \text { in direction } k \\
\text { TPHP } & =\text { typical peak hour passengers } \\
W & =\text { required width of pedway }
\end{array}
$$

${ }^{2}$ Ibid, pp. 74-84. 


\subsection{2 - Numerical Example of Pedestrian Flow Analyses}

As an example, assume that for an airport containing a finger-pier concourse similar to the one illustrated in Figure 8.1(a), the value of $S_{2,4}\left(y_{0}\right)=.45$ and the typical peak hour passenger volume is forecast to be 5,000 passengers. The value of $P$ in terms of the required width $W$ is thus simply

$$
P=\frac{37.5}{W} \text { passengers per foot width per minute }
$$

In general, the average flow volume can be expressed graphically as inversely proportional to the required width, as illustrated in Figure 8.4.

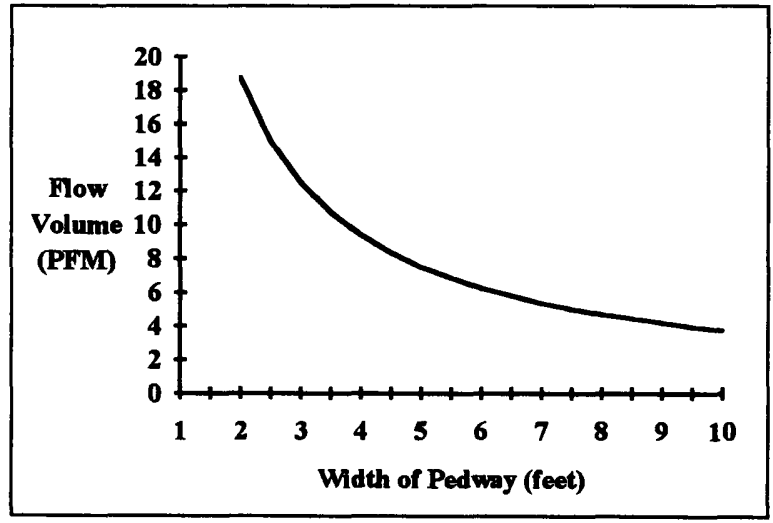

Figure 8.4 : Relationship of $P$ to $W$

To determine the width of the pedway necessary to achieve a desired minimum level of service, we simply read the leftmost value of $M$ (in Figure 8.3) corresponding to the desired standard (A-F), and use the appropriate value of $P$ in Equation 8.6 to obtain $W$ (alternatively, if we are given the value of $W$, we can use Equation 8.6 and Figure 8.3 to anticipate the expected level of service). For example, the upper and lower bounds needed to obtain a "B" level of service for a walkway (using the data given previously) are 5.4 and 3.8 feet, respectively. The corresponding upper and lower bounds for a stairway are 7.5 and 5.4 feet, respectively. 


\section{3 - Congestion in an Area}

Section 8.2 described methods of estimating potential passenger congestion across a linear section of a passenger building (i.e. screen lines). Such information is useful when determining the required widths of walkways and stairways needed to provide a given level of service. This section extends our analysis to two dimensions and describes methods of estimating potential passenger congestion within a specified area of a passenger building. As before, we can use these estimates to determine the level of service afforded passengers (and/or well-wishers), this time according to design standards such as those specified by the International Air Transport Association. A discussion of these standards and their use by current practitioners follows.

\subsection{1 - IATA Level of Service Parameters}

Research conducted by the AACC/IATA Working Group on Traffic Peaks [IATA 1981] led to the conclusion that "standard definitions should be used in evaluating levels of service and airport capacity." Specifically, the working group sought to establish a methodology for determining the capacity of an airport (including individual airport subsystems), which took into account the level of service to be provided to passengers, which could then be compared with the anticipated peak demand to identify capacity limitations.

The working group identified three general categories of airport subsystems to study; namely Processors, Reservoirs, and Links, defined as follows :

Basically, Processors involve an essential monetary, regulatory, or security transaction between the passenger and a person or machine (e.g. ticketing); Reservoirs are waiting or queueing areas (e.g. holdrooms); and Links allow for the movement of occupants from one subsystem to another (e.g. corridors, escalators). [p. 8]

Depending on which subsystem is being analyzed, three fundamental measures of capacity can be used to estimate potential congestion; namely Static, Dynamic and Sustained Capacity, defined as follows : 
Static Capacity is used to describe the storage potential of a facility or area, and is usually expressed as the number of occupants which a given area will hold at any one moment. It is a function of the total usable space available and the Level of Service provided, i.e., the amount of space each occupant is to have.

Dynamic Capacity refers to the maximum processing of flow rate of pedestrians (i.e. occupants) through a subsystem per unit time. The actual time unit selected as the measurement index (e.g. minutes, hours) depends on the nature of the operation.

Sustained Capacity is used to describe the overall capacity of a subsystem to accommodate traffic demand over a sustained period within the space and time standards of a particular Level of Service. It is thus a measure of the combine Dynamic and Static Capacities of the Processors, Reservoirs and Links. [p. 8,9]

For estimating potential congestion within an area, the static capacity can be calculated based on spatial requirements established for each different level of service. The spatial requirements and definitions of each level of service are provided in Tables 8.2 and 8.3. Note that although the description of each individual service level remains the same, subsystems have differing spatial requirements.

As an example, let us assume that we wish to achieve a "B" level of service for two different subsystems, a check-in area and a waiting room, both of the same size, $1500 \mathrm{~m}^{2}$. The maximum number of individuals who could occupy the respective areas (at any one time) and still achieve this level of service is simply :

Check-in Area $=1500 / 1.4=1,071$ individuals
Waiting Area $=1500 / 2.3=652$ individuals

The number of individuals within a given airport subsystem can change quickly, however, and thus it is important to study the behavior of passenger (and well-wisher) accumulation over time, rather than just at one instant. In Section 8.3.2, we present a more general model for estimating potential congestion within an area, which accounts for this time dependent nature of passenger accumulation. 
Table 8.2: Level of Service Framework

\begin{tabular}{cl}
\hline Level & Description of Service Level \\
\hline A & $\begin{array}{l}\text { Excellent level of service; condition of free flow, no delays; } \\
\text { excellent level of comfort. }\end{array}$ \\
B & $\begin{array}{l}\text { High level of service; condition of stable flow, very few delays; } \\
\text { high level of comfort. }\end{array}$ \\
C & $\begin{array}{l}\text { Good level of service; condition of stable flow, acceptable delays; } \\
\text { good level of comfort. }\end{array}$ \\
D & $\begin{array}{l}\text { Adequate level of service; condition of unstable flow, acceptable } \\
\text { delays for short periods of time; adequate level of comfort. }\end{array}$ \\
E & $\begin{array}{l}\text { Inadequate level of service; condition of unstable flow, unacceptable } \\
\text { delays; inadequate level of comfort. }\end{array}$ \\
F & $\begin{array}{l}\text { Unacceptable level of service; condition of cross-flows, system } \\
\text { breakdown and unacceptable delays; unacceptable level of comfort. }\end{array}$ \\
\hline
\end{tabular}

Table 8.3 : Examples of Congestion Standards

Level of Service Standards (Square Meters per Occupant)

\begin{tabular}{|c|c|c|c|c|c|c|}
\hline Sub-System & A & B & $\mathrm{C}$ & $\mathrm{D}$ & $\mathrm{E}$ & F \\
\hline \multicolumn{7}{|l|}{ No Baggage } \\
\hline $\begin{array}{l}\text { Holdroom } \\
\text { Pre-Inspection }\end{array}$ & 1.4 & 1.2 & 1.0 & .8 & .6 & \\
\hline With Baggage & & & & & & $\begin{array}{l}S \\
Y\end{array}$ \\
\hline $\begin{array}{l}\text { Check-in } \\
\text { Bag Claim Area } \\
\text { (Without Device) }\end{array}$ & 1.6 & 1.4 & 1.2 & 1.0 & .8 & $\begin{array}{l}\mathrm{S} \\
\mathrm{T} \\
\mathrm{E} \\
\mathrm{M}\end{array}$ \\
\hline Circulation & & & & & & \\
\hline Wait/Circulate & 2.7 & 2.3 & 1.9 & 1.5 & 1.0 & \\
\hline
\end{tabular}




\section{Current Practitioners / Use of IATA Parameters by Current Practitioners}

The use of the IATA level of service parameters by current practitioners varies significantly throughout the industry. Policy making authorities, such as Transport Canada, use the IATA standards rigorously as part of their capital budgeting process. According to Dr. Lloyd McCoomb, then Director General of Safety and Technical Services at Transport Canada (until April 1994),

Level of service design parameters, expressed in units of space per passenger, are used in planning of terminals at our airports and are contained in our planning and design manuals ... They are also used, along with the passenger processing rates, in arriving at the total space requirements for large air terminals using our Terminal Flow Simulation Model.

Our capital project approval system calls for conformity with the Transport Canada policies, standards and guidelines ... The approval system requires that the air terminal building project submissions include passenger traffic forecasts, the size of the proposed new building or of the expansion of an existing building, including areas reserved for the functional (processing) elements. These are then compared to the design guidelines to ascertain that the proposed expenditure will result in the optimum airport improvement using the least amount of funds. ${ }^{3}$

Local airport authorities, such as the Port Authority of New York and New Jersey (PANYNJ), make extensive use of the IATA standards, in part as input to "in-house" computer analysis tools that use these standards to calculate the capacities of existing and new passenger building designs. According to Tom Lee (of the PANYNJ), "We use the IATA level of service standards every day in analyzing each function, or each step of a passenger's progression through our facilities." 4 The Milan Airport Authority made extensive use of the IATA level of service parameters in the design of the new terminal of the Malpensa Airport. This new terminal is expected to service 16-20 million passengers per year, and was designed to provide a "B"-level of service during peak periods. 5

On the other hand, private consulting companies, such as JKH Mobility Services (a subsidiary of Kimley-Horn and Associates of Raleigh, North Carolina), Thompson Consultants International of

\footnotetext{
${ }^{3}$ Excerpts from letter to the author on March 10, 1994.

${ }^{4}$ Excerpt from telephone conversation with author on April 5, 1994.

${ }^{5}$ From conversation on April 20, 1994 with Professor Amedeo Odoni, who acted as a consultant on the Malpensa project.
} 
Burbank, California, and P \& D Technologies of Orange County, California prefer to use proprietary simulation models (which contain their own level of service parameters) when designing the interiors of passenger buildings. ${ }^{6}$ According to one consultant,

The IATA standards tend to be relatively crowded. By the time you get to their level of service " $F$ ", it's a pretty intolerable condition. When you're trying to design (or help others design) a new terminal, or you are evaluating an existing design, you don't want to get anywhere near level "F". Airport authorities always want us to design for level of service "A".

Typically, we'll base our analyses on very detailed, time-history simulations, where we will create a model of the facility, using specific flight schedules that they anticipate having there ... When you look at things like sizing of corridors and sizing how many escalators you need for the flows of passengers that are being served, generally those [IATA standards] are a little harder to define and interpret.

Frequently what you'll find is, in the deliberation on the adequacy of the design of a new facility, you'll look at the problem in several different ways, just as a consistency check among different schemes. The IAT A level of service is typically one that is at least considered, but it is not the one that is relied upon. ${ }^{7}$

Thus, there is a great disparity among current practitioners in the way the IATA level of service standards are applied. The presence of "in-house" standards among private consulting firms is likely to result from competitive advantages to differentiation within the industry, whereas more "public" authorities may wish simply to maintain a consistent "congestion" vocabulary. In the next section we describe a general model for estimating congestion within an area. Later, as a proof of concept, we apply this model (in conjunction with the IATA level of service standards given in Tables 8.2 and 8.3) to a numerical example of Terminal $\mathrm{E}$ at Boston Logan Airport.

\subsection{2 - General Model for Estimating Area Congestion}

The general framework for estimating potential congestion across a screen line is based on principles of fluid dynamics related to maximum flow. Passengers traveling (in one or two directions) across a screen line are considered in the congestion analysis only at the instant they cross the line. The

\footnotetext{
${ }^{6}$ From private telephone conversations in April 1994.

${ }^{7}$ Excerpts from telephone conversation with author on April 7, 1994.
} 
capacity (over time) of the walkway is in some sense considered infinite, constrained only by the number of passengers per foot per minute (PFM) who can cross for a given level of service. In extending our analysis to congestion within an area, however, we can no longer assume that the capacity of the system is infinite over time. Similar to a queueing system, individuals enter an area of a passenger building and remain there for some time, usually until they are "served". In the case of an airport. customers are usually considered "serviced" when they are allowed to leave the system. In a gate holdroom, passengers can leave the system once boarding commences. In a baggage claim area, passengers can leave once they retrieve their bags.

As mentioned previously, the amount of floor space available to an individual naturally depends upon the number of people present within that area at a given time. This number is, in turn, directly related to the flow of passengers into and out of the area. Newell [1971] studied various applications of queueing theory using approximations based on principles of fluid dynamics. He defined the functions $A(t)$ and $D(t)$ to represent the cumulative number of arrivals and departures (respectively) by time $t$, at a queueing system ${ }^{8}$.

He also defined the arrival and departure rates, $\lambda(t)$ and $\mu(t)$, which are simply the first derivatives of $A(t)$ and $D(t)$, respectively. Arrival rates (as well as departure rates) can vary by flight based on time of day, day of week, even season. Thus, we could define flight-specific parameters $\lambda_{i}(t)$ and $\mu_{i}(t)$ for each flight $i$. For simplicity, however, let us assume that the flights we consider are sufficiently similar to keep $\lambda(t)$ and $\mu(t)$ the same for each flight.

From the previous definitions, we can derive simple relationships to determine the number of individuals present in the system at any given time $t$. For example, we can express $A(t)$ and $D(t)$ as

$$
A(t)=\int_{0}^{t} \lambda(t) d t
$$

\footnotetext{
${ }^{8}$ In Newell's notation, $D(t)$ denotes the cumulative departure function when the service time, $S$, is zero.
} 
and

$$
D(t)=\min \left[\int_{0}^{t} \mu(t) d t, A(t)\right]
$$

$D(t)$ is the minimum of the two components as there cannot be more departures than arrivals into a system (unless there are individuals present in the system at time $t=0$ ). Newell [1971] also defined a function $Q(t)$ to represent the number of individuals present in the system at time $t$, such that

$$
Q(t)=\max \left[0, \int_{0}^{t} \max (0,[\lambda(t)-\mu(t)]) d t\right]
$$

or

$$
Q(t)=A(t)-D(t)+Q(0)
$$

where

$Q(t)=\quad$ Number of individuals present in the system at time $t$

$A(t)=$ Cumulative number of arrivals into the system by time $t$

$D(t)=$ Cumulative number of departures from the system by time $t$

$Q(0)=\quad$ Number of individuals already in the system at time $t=0$

For our purposes, $Q(t)$ represents the number of "dwellers" (passengers and/or well-wishers) who occupy a given area (i.e. the system) at time $t$. For planning purposes, we are interested in the anticipated behavior of $Q(t)$ throughout the day, and the identification of the maximum value of $Q(t)$ over the time interval under consideration. This maximum value, $Q^{*}(t)$, can be used to determine the level of service afforded individuals, as specified by such design standards as those described in Section 8.3.1. To determine the anticipated level of service, we simply divide the total area of the given space by the value of $Q^{*}(t)$. An example of this calculation is provided in the case study in Section 8.4.3. 


\section{4 - Case Study - Boston Logan International (Terminal E)}

This section applies some of the models presented in this chapter to the International Terminal (E) at Boston Logan Airport. The input data used in our models was obtained from a report [FTA 1990] summarizing the results of a detailed simulation analysis performed as part of a landside demand/capacity analysis done by Flight Transportation Associates, an aviation consulting firm located in Cambridge, Massachusetts. The report includes an analysis of three hypothetical layouts for the security checkpoints in Terminal E. In our analysis, we will study "Security-Check-Plan \#3" (Figure 8.5), described in the report as follows:

Gates 1 and 2 would have two [security] stations in front of the gate entrances while gates 3 and 4 would have another two stations. No passenger waiting area is available beyond the security checks. For gates 7 and 8 , one station is available in front of each gate entrance and no waiting area is available behind the stations. Access to the area between gate 7 and 8 is possible after security checks. This design allows shared use of the two security machines between gates 7 and 8, for a total of eight machines. [p. 2]

\subsection{1 - General Description of Terminal E}

For most analyses, Terminal E is typically separated into two parts, domestic and international operations, as illustrated in Figures 8.6 and 8.7. Currently, however, it is more appropriate to consider the two parts as Northwest (Airlines) operations, and "Other Airline" operations, since international passengers traveling on Northwest use what is generally referred to as the domestic portion of the building. Enplaning or originating passengers traveling on Northwest are assumed to follow the paths specified in Figure 8.6, which all include the use of the stairway located nearest to the ticket counters (denoted TC in the figure). Likewise, originating passengers on the Other Airlines are assumed to follow the paths specified in Figure 8.7, which all include the use of the stairway located nearest to the ticket counters on the other side of the building. 

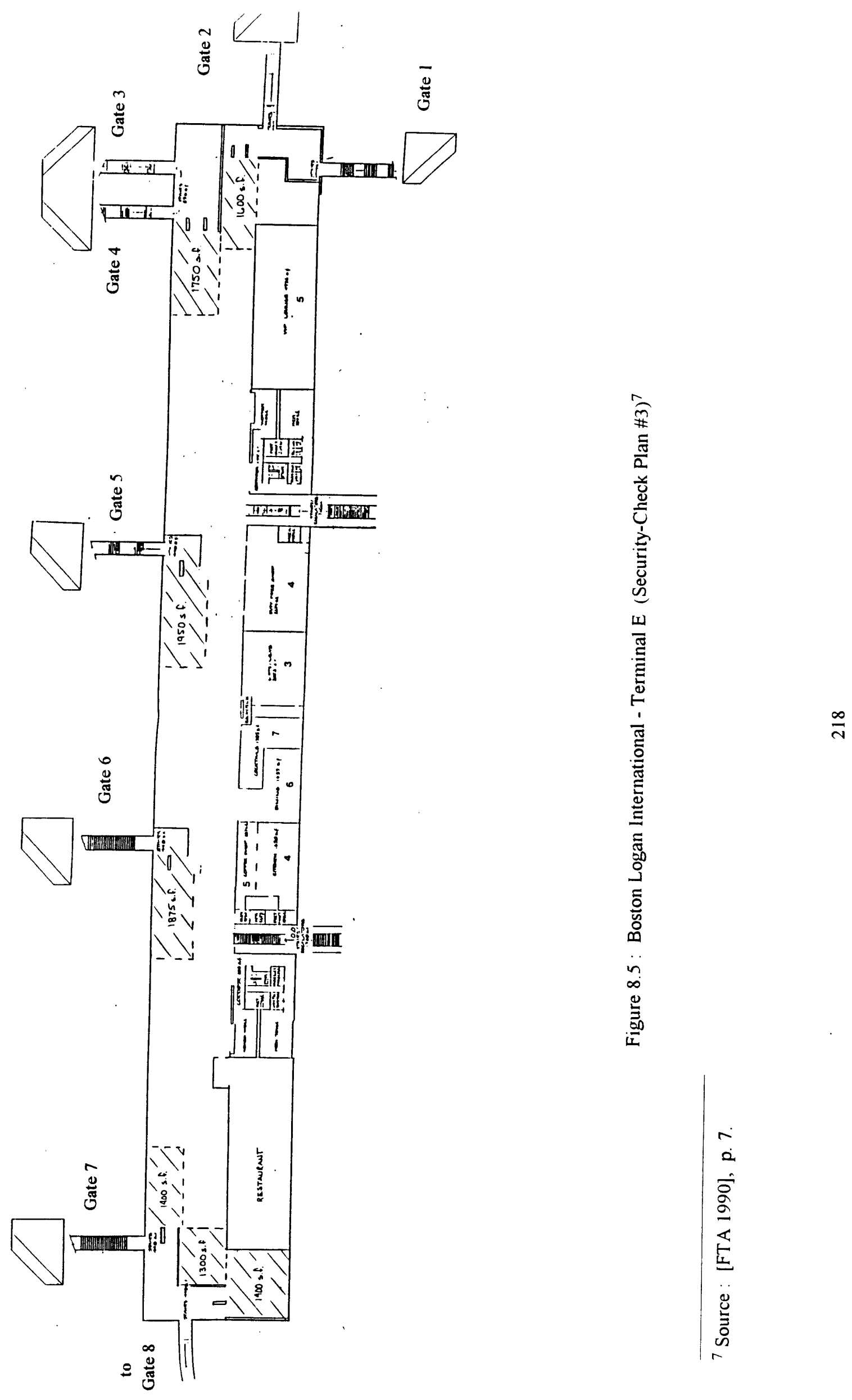


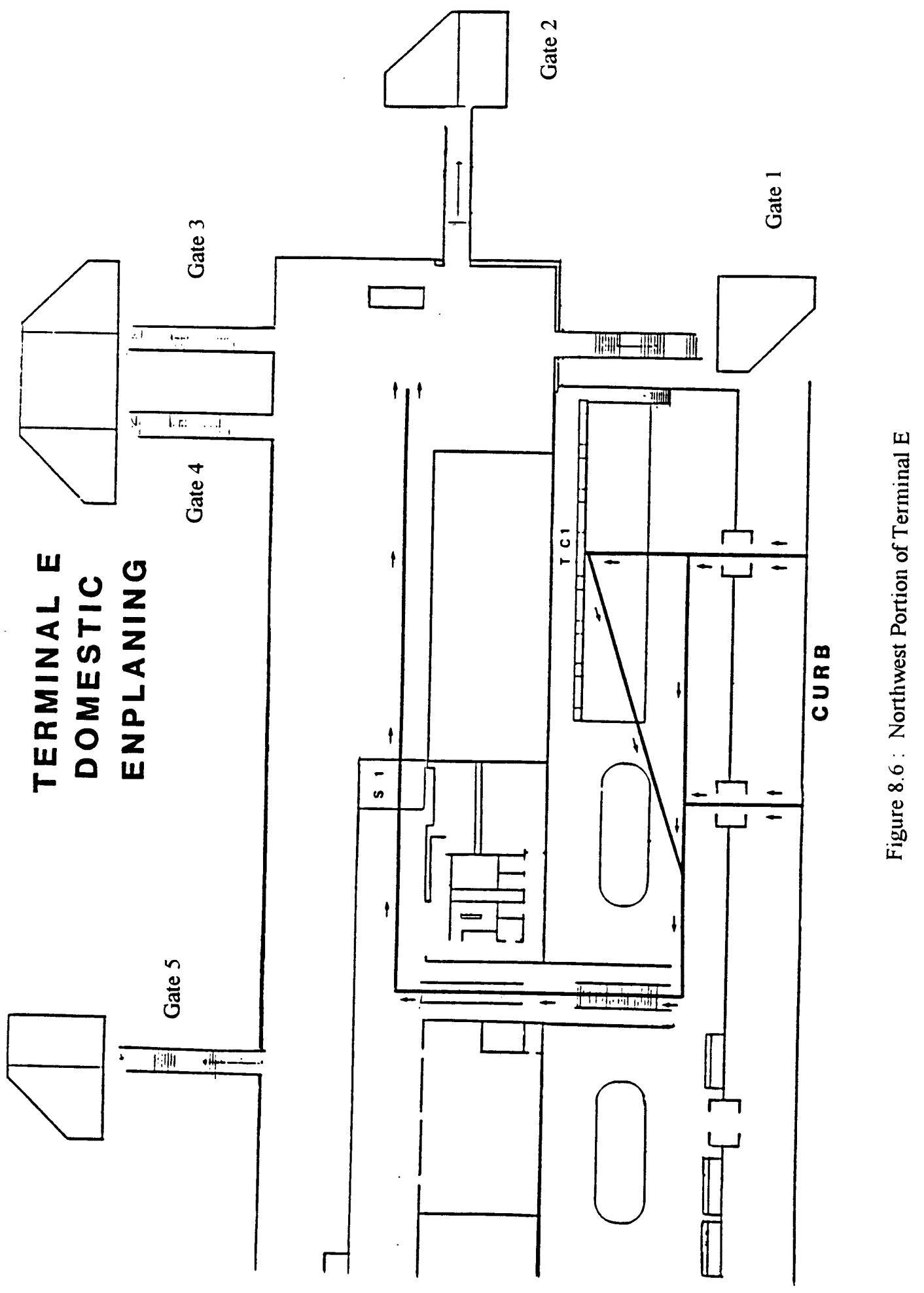

$\frac{a}{2}$ 


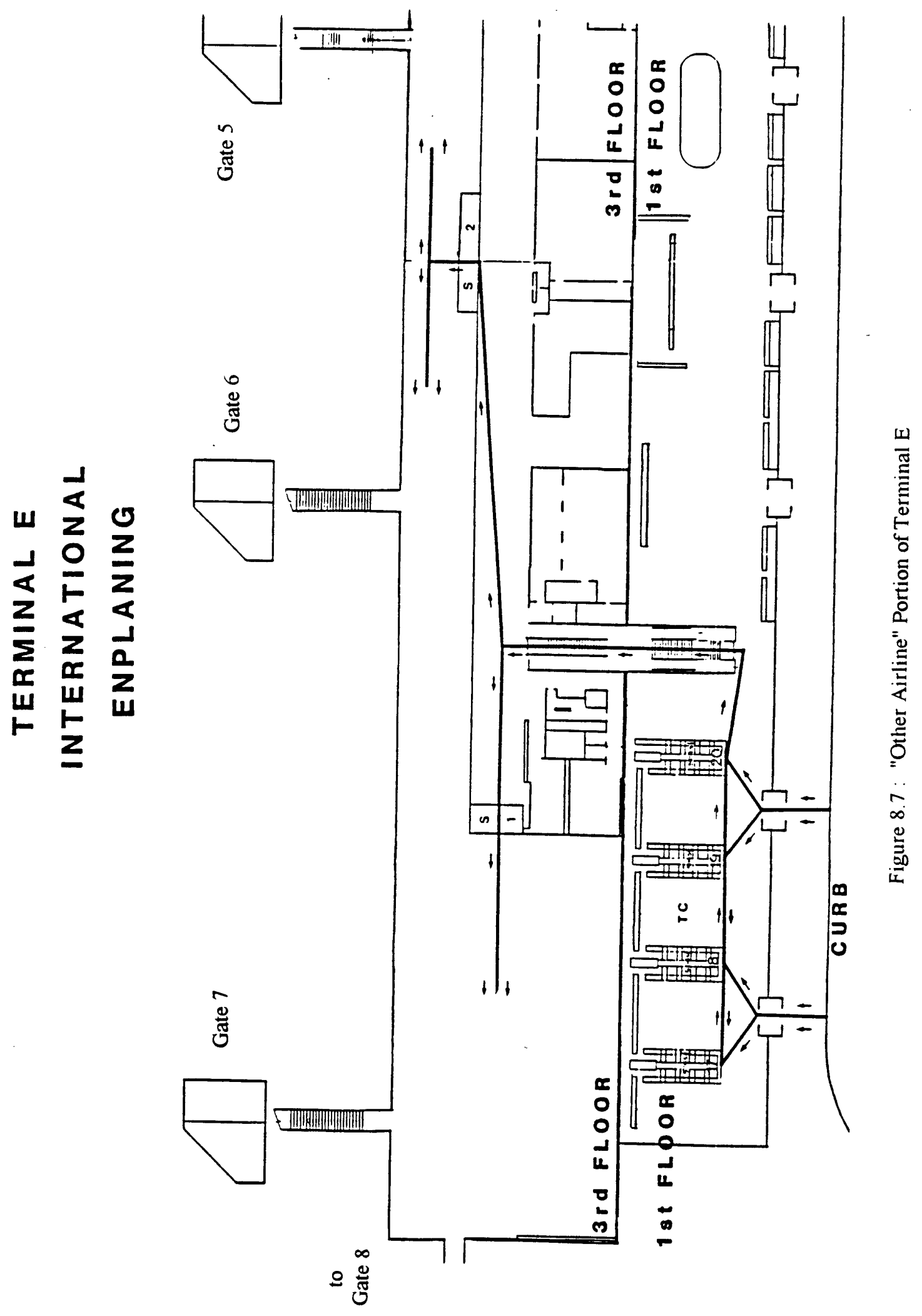

สิ 
Appendix A lists the relevant input information used for the detailed simulation analysis performed by FTA. Table A.1 shows a sample flight schedule, including departure times and equipment type (including seating capacity), and Table A.2 lists the average load factor assumed for all flights. Table A.3 lists the cumulative arrival curves for international and domestic (originating) passengers, rounded to the nearest 5-minute interval for convenience. Table A.4 lists, in cumulative percentage form, the number of well-wishers associated with each originating passenger. From this table, we can calculate the expected number of well-wishers per passenger (approximately .33). Finally, Table A.5 lists the passenger mix assumptions made for Northwest and the Other Airlines.

\subsection{2 - Congestion Across a Line: Required Stairway Widths}

This section estimates the required width of the stairways mentioned in the previous section using screen lines placed at the foot of each stairway. From the data provided in Appendix A, it is possible to construct the arrival rates of individuals (originating passengers and their well-wishers) into the system (Terminal E). For our analysis, we separate the building into the two sections described previously, Northwest operations, and Other Airline operations. The results of our analyses follow.

\subsubsection{1 - Other Airline Operations}

From the cumulative arrival distribution for originating international passengers (Table A.3), it is possible to estimate the number of passenger (and well-wisher) arrivals into the system during a given time interval $t$. For simplicity, let us assume this time interval is five minutes; further, let us assume that passenger arrivals within each portion of the cumulative arrival curve are constant (e.g. over a fifteen minute interval, if 30 percent of the passengers are expected to arrive, we assume that 10 percent of them arrive during each of three 5-minute intervals). From the data provided in Appendix A, we can estimate the number of individuals arriving into the system associated with each flight, and the approximate number of those individuals arriving within each 5-minute time interval. 
As an example, consider Flight LH 423, departing at 18:00 hrs. Based on an average load factor of 80 percent, a passenger mix of 90 percent originating passengers and an expected number of wellwishers of .33 , the number of individuals expected to "arrive" for this flight is

$$
282(.80)(.90)(1.33) \cong 270 \text { individuals }
$$

Passengers and their well-wishers are assumed to begin arriving at 15:30 hrs, 150 minutes prior to departure (Table A.3). The "simulated" cumulative arrival curve for this flight (based on the above mentioned data) is illustrated in Figure 8.8. Note that for consistency we must adopt the assumption (used in the FTA report) that the data from Table A. 3 can be used to describe arrivals into the waiting area in addition to arrivals at the curb (even though this may not be the case). As such, they are "assumed" or simulated arrival curves, rather than measured ones.

If we repeat our analysis for all "Other Airline" flights (assuming the same arrival pattern for each flight), and sum the number of arrivals across flights for each time interval, we obtain a total cumulative arrival curve for all individuals entering the "Other Airline" portion of Terminal E. This cumulative arrival curve is illustrated in Figure 8.9.

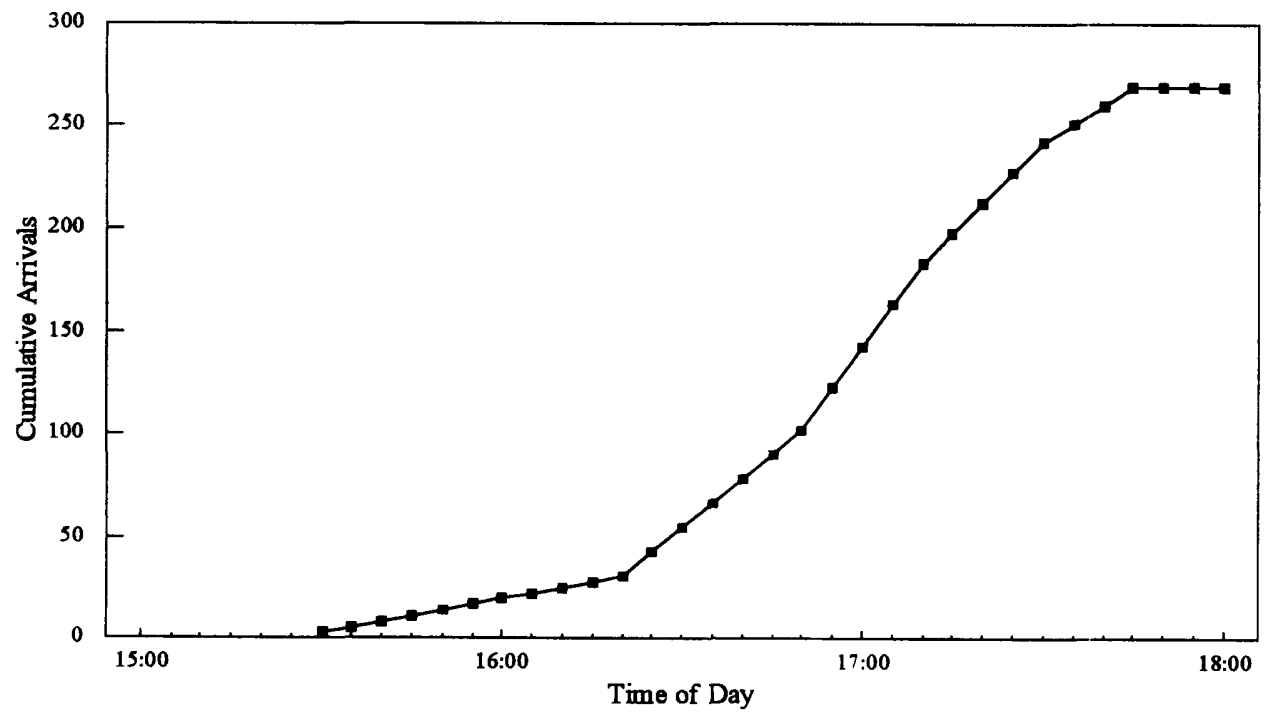

Figure 8.8 : Simulated Cumulative Arrival Curve for LH 423 


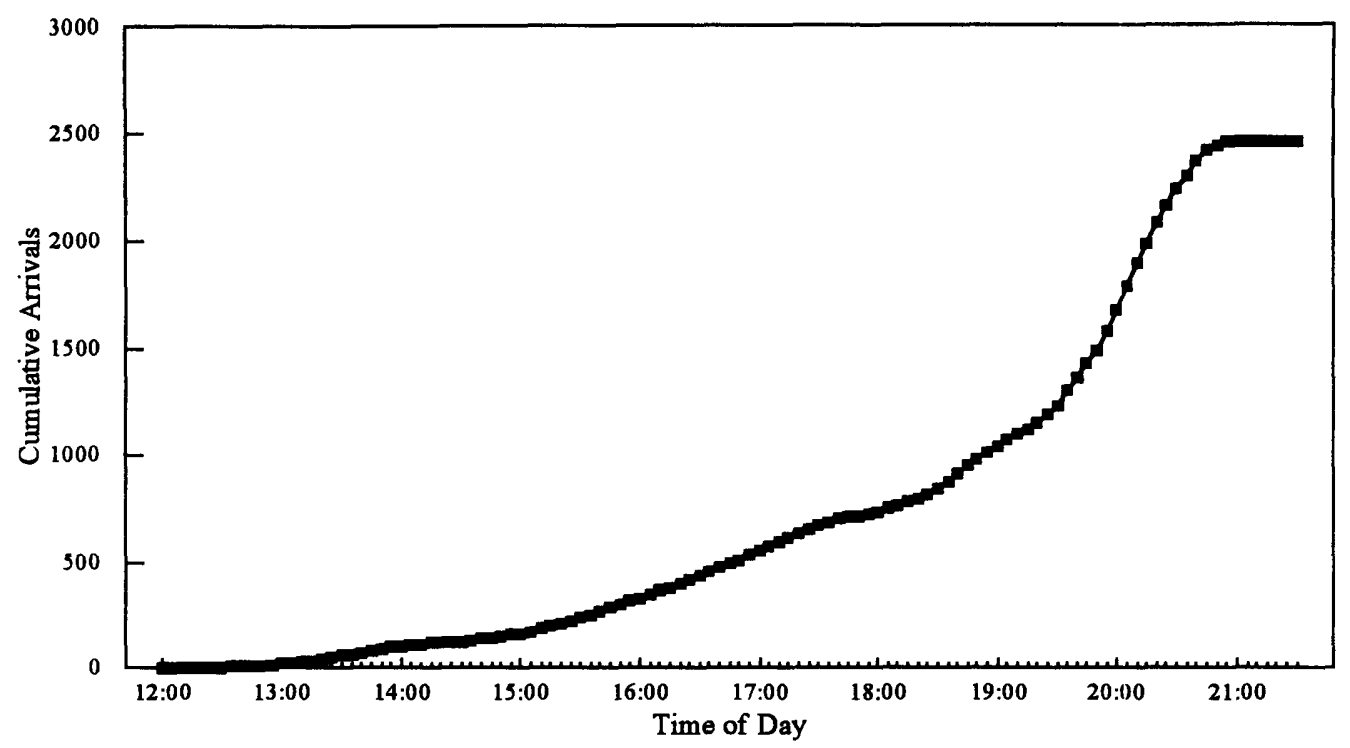

Figure 8.9 : Simulated Cumulative Arrival Curve for All "Other Airline" Flights

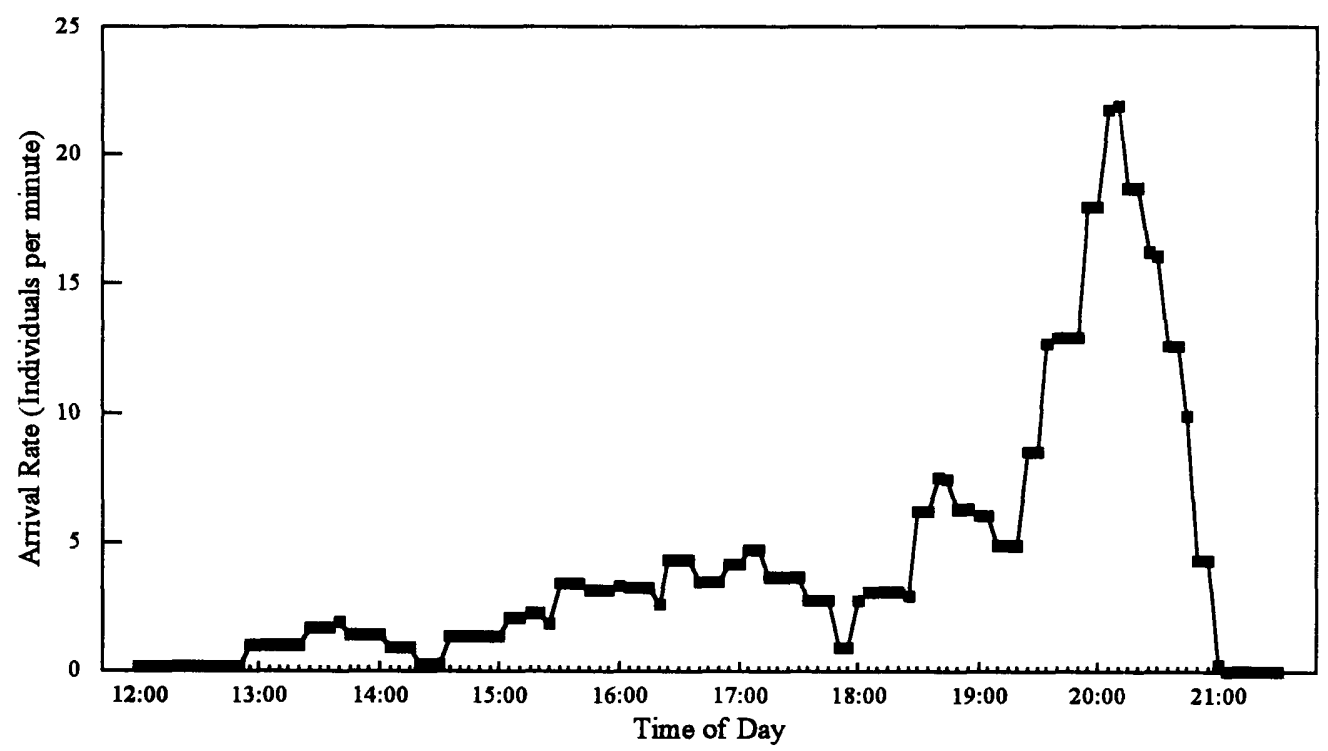

Figure 8.10 : Arrival Rate Curve for Individuals on all "Other Airline" Flights 
From Figure 8.9 we see the total number of arrivals into the system is approximately 2,450 individuals; however, the individuals arrive over an 8-hour time period at different rates throughout the afternoon and evening. In order to determine the required width of the stairway, we need to examine the arrival rate curve, or the first derivative of the cumulative arrival curve. Figure 8.10 illustrates the arrival rate curve corresponding to the cumulative arrival curve shown in Figure 8.9.

The maximum value of the arrival rate curve is 22 individuals per minute, occurring at 20:10 hrs. Using an expression equivalent to Equation 8.5, it can be shown that in order to achieve an " $\mathrm{A}$ " level of service (less than five passengers per foot per minute) for individuals ascending the stairway, the required width would need to be at least 4.4 feet. The width of the staircase available for ascending individuals is more that twice that (approx. $14 \mathrm{ft}$ ), thus easily providing an " $\mathrm{A}$ " level of service. For the given flight schedule, then, the staircase available for "Other Airline" originating passengers (and their well-wishers) is more than adequate to provide an "Excellent" level of service.

\subsubsection{2 - Northwest Operations}

We can perform a similar congestion analysis for the portion of Terminal $E$ used for Northwest operations. The only difference is that several of the Northwest flights listed in Table A.1 are domestic flights, and thus we must apply the domestic cumulative arrival curve to passengers and well-wishers associated with those flights. Figure 8.11 illustrates the total, simulated cumulative arrival curve associated with all Northwest flights (domestic and international). Note that unlike the cumulative arrival curve for the Other Airlines, the Northwest curve shows a significant number of arrivals during the early portion of the afternoon, corresponding to their domestic departures. Note also that the maximum value of the Northwest cumulative arrival curve is 3,470 individuals, 40 percent more than for the Other Airlines.

We can again find the arrival rate curve, this time for Northwest passengers and their wellwishers, by taking the first derivative of the cumulative arrival curve. This curve is illustrated in Figure 8.12. As mentioned previously, there is an early afternoon peak associated with domestic departures, as 
well as an evening peak associated with international departures. Note that the maximum value of the Northwest arrival rate curve is only 16 individuals per minute, occurring at 19:25 hrs. If we apply a similar analysis to the screen line located at the foot of the staircase in the Northwest portion of the building, the width needed to achieve an "A" level of service can be shown to be only $3.1 \mathrm{ft}$ (compared to 4.4 $\mathrm{ft}$ on the Other Airline side), even though this side of the building "witnesses" over 1000 individuals more during the same 8-hour period.

This example demonstrates the effects of demand peaking on potential congestion. The Northwest demand, although greater in absolute volume, is spread out over two peaks, one in the early afternoon and one in the evening. The maximum arrival rate of Northwest passengers (and their wellwishers) over the entire interval is actually lower than the maximum rate corresponding to the singlepeaked "Other Airline" demand. In the next section, we extend our analysis to consider congestion within an area, and show how this "peakiness" of demand affects spatial requirements as well.

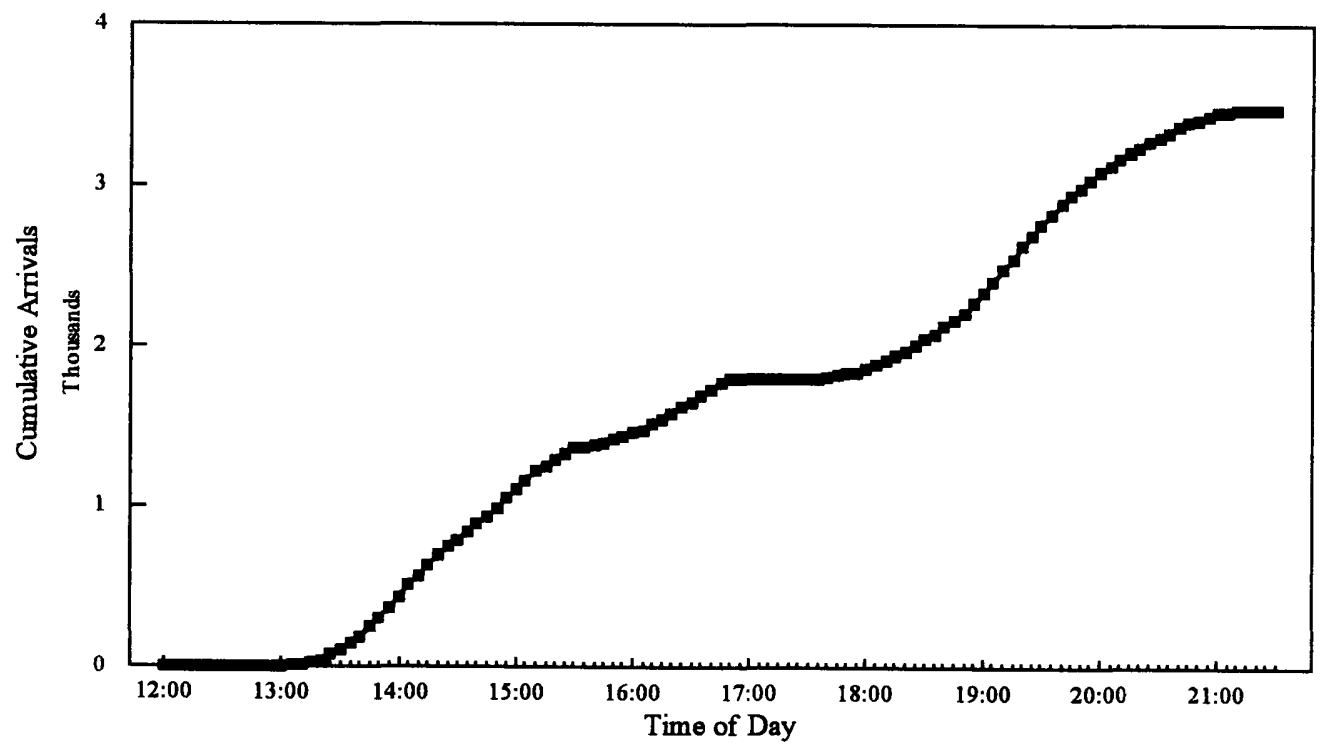

Figure 8.11 : Simulated Cumulative Arrival Curve for All Northwest Flights 


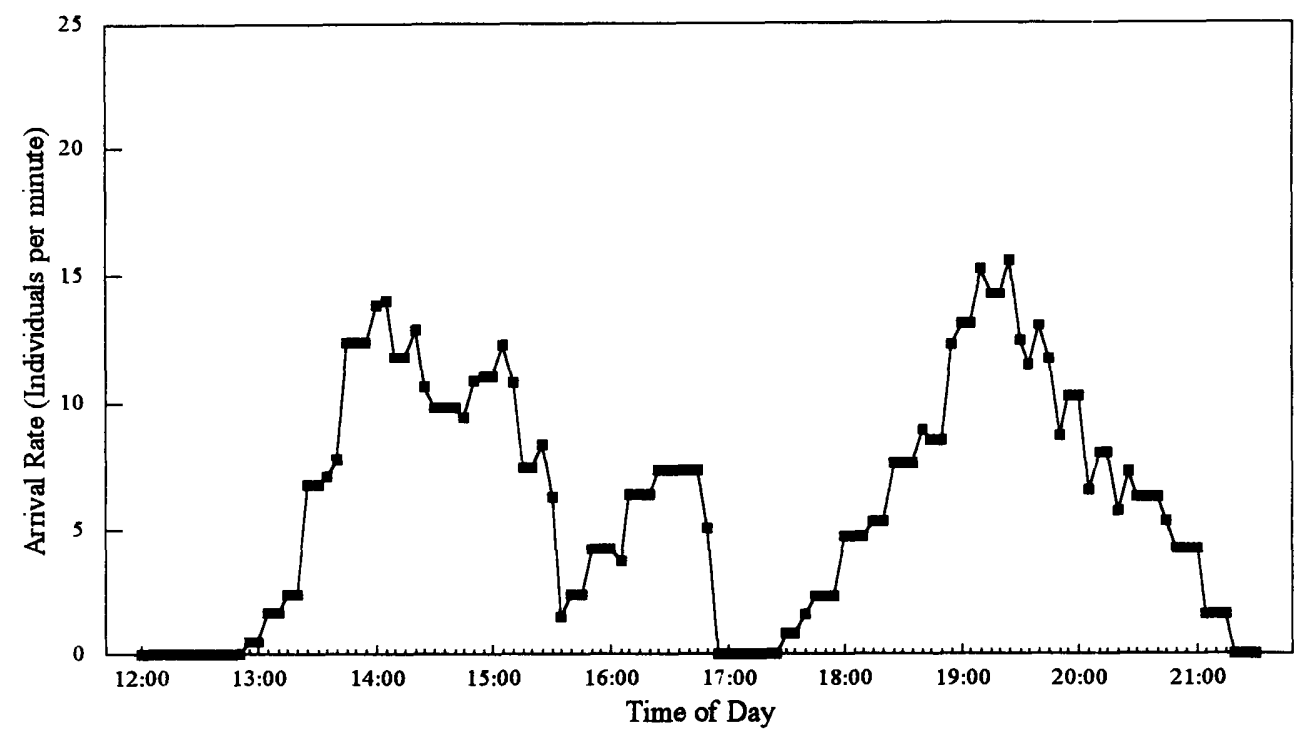

Figure 8.12: Arrival Rate Curve for All Northwest Flights

\subsection{3 - Congestion in an Area}

This section employs the general flow model described in Section 8.3.2 to determine spatial requirements for "dwelling" individuals once they have ascended the respective staircases (described in the previous section). The Reservoir, in this case, is the "net available waiting area" or unused floor space on the third floor of Terminal E, after taking out queueing areas and the area occupied by facilities such as restaurants, shops, restrooms, and V.I.P. lounges.

As mentioned previously, the FTA [1990] report studied different, hypothetical security-check plans in Terminal E. One of the measures of performance used in their analysis was the level of service afforded passengers and well-wishers within the net available waiting area. The arrival rate, $\lambda(t)$, or "flow into" the reservoir can be approximated by the arrival rate curve of originating passengers and their well-wishers, calculated in the previous section. The departure rate, $\mu(t)$, or "flow out" of the reservoir is composed of two processes. The first is the boarding of departing passengers onto aircraft; the second is the departure of well-wishers from the area once boarding has begun. 
Depending on how far well-wishers can accompany ticketed passengers (due to security restrictions), the departure of well-wishers may actually occur well before passenger boarding commences; however, if we are estimating spatial requirements based on maximum passenger accumulation, it may be appropriate to assume that well-wishers begin to depart as late as possible, i.e. at the same time passenger boarding commences. As illustrated in Security-Check Plan \#3 (Figure 8.5), passengers do not pass through security-checks until just before boarding the aircraft (recall that "no waiting area is available behind the [security] stations"). Thus, we will assume for our example that wellwishers do in fact accompany passengers to the waiting area and do not depart until the passengers board. Both components of $\mu(t)$ can thus be approximated by some maximum passenger boarding rate, $b$.

\subsubsection{1 - Other Airline Operations}

Consider again the "Other Airline" portion of Terminal E (Figure 8.6), with the cumulative and arrival rate curves illustrated in Figures 8.9 and 8.10, respectively. Let us assume that passenger boarding for each international flight begins at 35 minutes prior to the scheduled departure time. Paullin and Horonjeff [1969] and Wirasinghe and Shehata [1988] have used a passenger boarding rate, $b$, of 840 passengers per hour when analyzing spatial requirements of departure lounges. The number of passengers who could board within the allotted time thus exceeds the maximum number of individuals who arrive for any of the flights listed in Table A.1. This boarding rate translates to a maximum departure rate, $\mu(t)$, of 70 individuals per 5-minute interval. For each time interval, $t$, prior to departure, we thus use the minimum of $[\mu(t)=70, A(t)]$ for the "observed" departure rate.

Under such assumptions, we can obtain a total departure rate curve, $\mu(t)$, for all "Other Airline" flights listed in Table A.1. Such a curve is illustrated along with the corresponding arrival rate curve, $\lambda(t)$, in Figure 8.13. By integrating each curve, we can obtain the cumulative arrival and departure curves (Figure 8.14) as given in Equations 8.7 and 8.8. By summing the values of $A(t)$ and $D(t)$ for each time interval $t$, we can obtain the net passenger accumulation curve, $Q(t)$, for the "Other Airline" portion of the building. This curve is illustrated in Figure 8.15. 


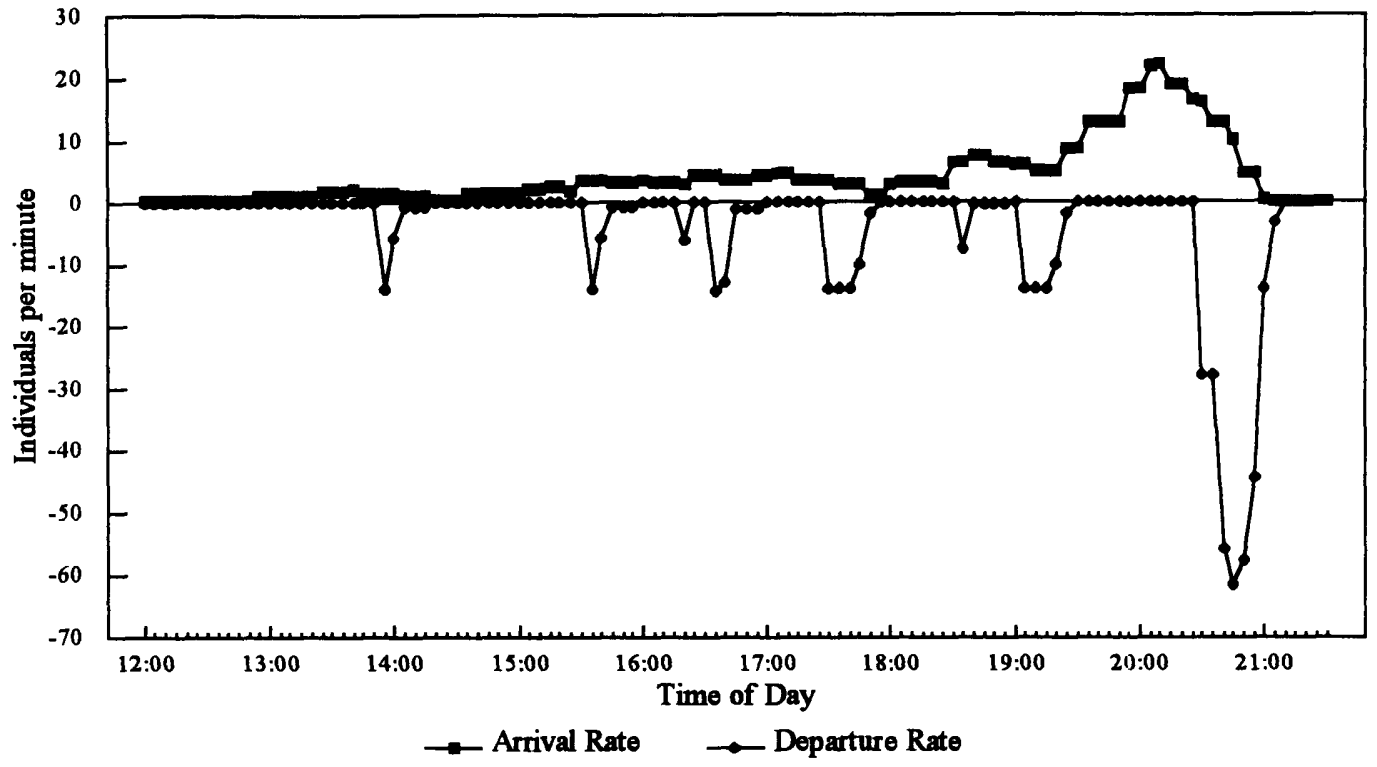

Figure 8.13 : Arrival and Departure Rate Curves for "Other Airline" Individuals

The maximum value of this curve, $Q^{*}(t)$, represents the maximum passenger accumulation over the entire time interval. From the curve we see that $Q^{*}(t)$ for this particular flight schedule is 1,160 individuals, occurring at 20:25 hrs.

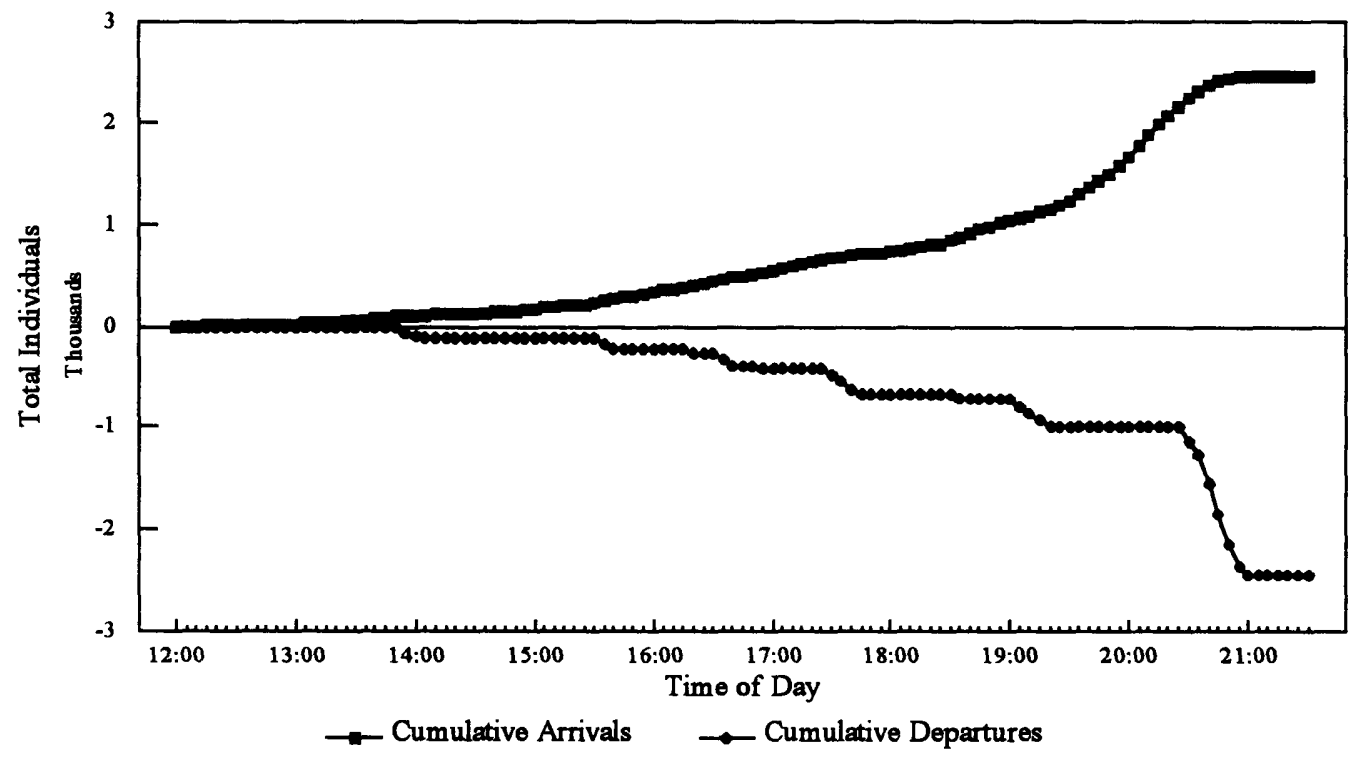

Figure 8.14: Cumulative Arrival and Departure Curves for "Other Airline" Individuals 
The FTA simulation study reported the following results from their analysis of Security-Check

Plan \#3 for Terminal E :

On the other-airline side, the net waiting area available after taking out queueing areas and the area occupied by the facilities is about 10,000 square feet. The simulation shows that during the evening peak hour there can be 1,100 passengers on the other-airline side at one time. This would result in service level "D" (based on IATA standards) at the gate holdroom. [p. 4]

For 10,000 square feet of available waiting area, a maximum accumulation of 1,164 individuals translates into 8.59 square feet per individual, which translates to a level of service "D" based on (English unit equivalent) IATA standards listed in Table 8.3 for waiting areas. Thus, we are able to achieve reasonably consistent results without the need for a detailed Monte Carlo simulation program.

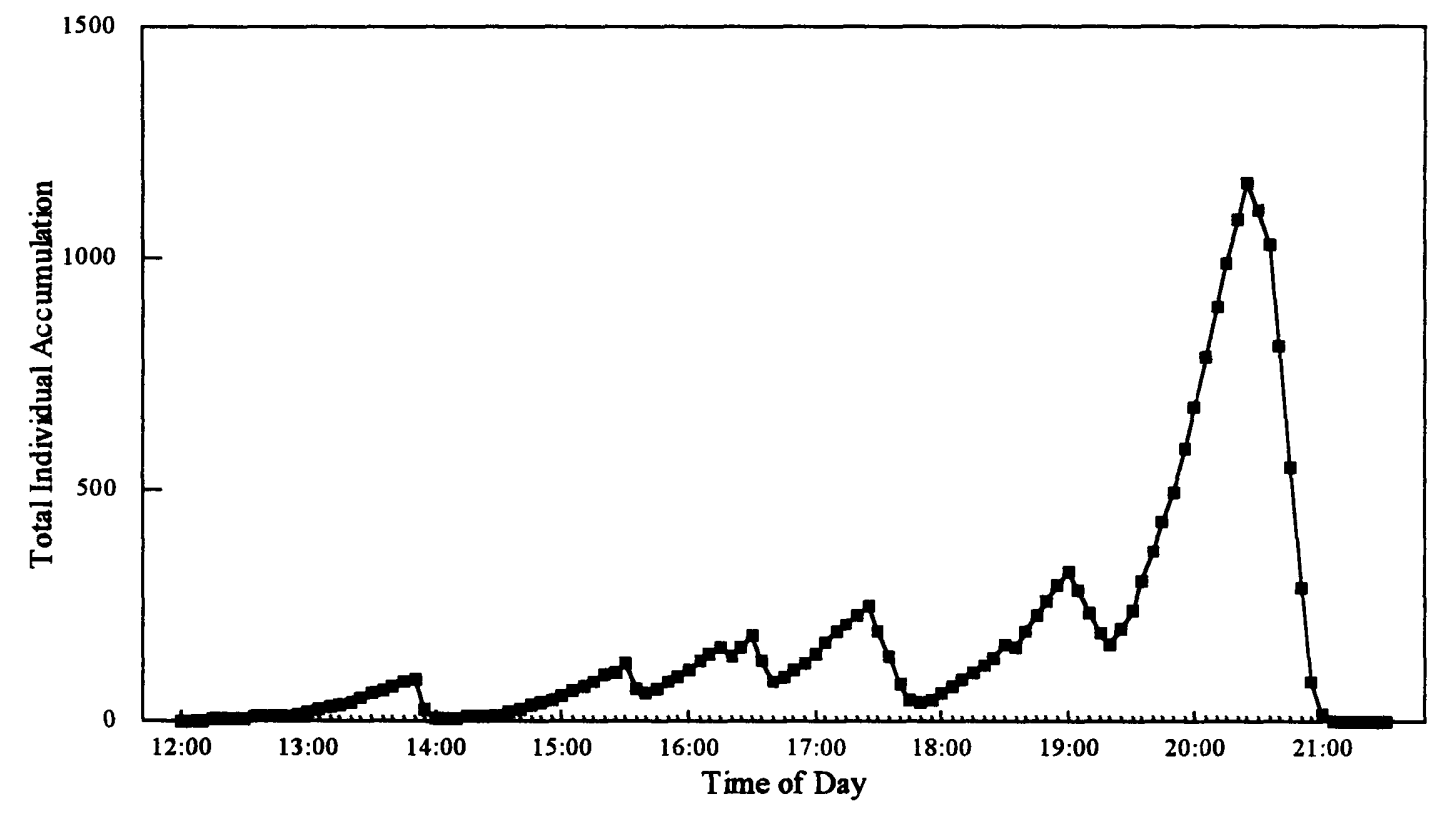

Figure 8.15: Total Individual Accumulation for "Other Airline" Flights 


\subsubsection{2 - Northwest Operations}

A similar congestion analysis can be performed on the Northwest portion of Terminal E to determine the maximum individual accumulation, and thus the level of service afforded passengers and their well-wishers, under Security-Check Plan \#3. The Northwest flight schedule, however, contains a number of domestic flights for which boarding typically begins closer to the time of departure than for international flights. Thus for those flights, we assume that passenger boarding and well-wisher departure begin at 20 minutes prior to departure time.

Under this and previous assumptions, a total departure rate curve, $\mu(t)$, can be obtained, and the arrival and departure rate curves can be integrated to obtain cumulative arrival and departure curves such as those illustrated previously in Figures 8.9 and 8.10. From these curves it is again possible to obtain a total individual accumulation curve, $Q(t)$, by summing the values of $A(t)$ and $D(t)$ for each time interval $t$. The resulting curve is illustrated in Figure 8.12 (shown in the same scale as Figure 8.11).

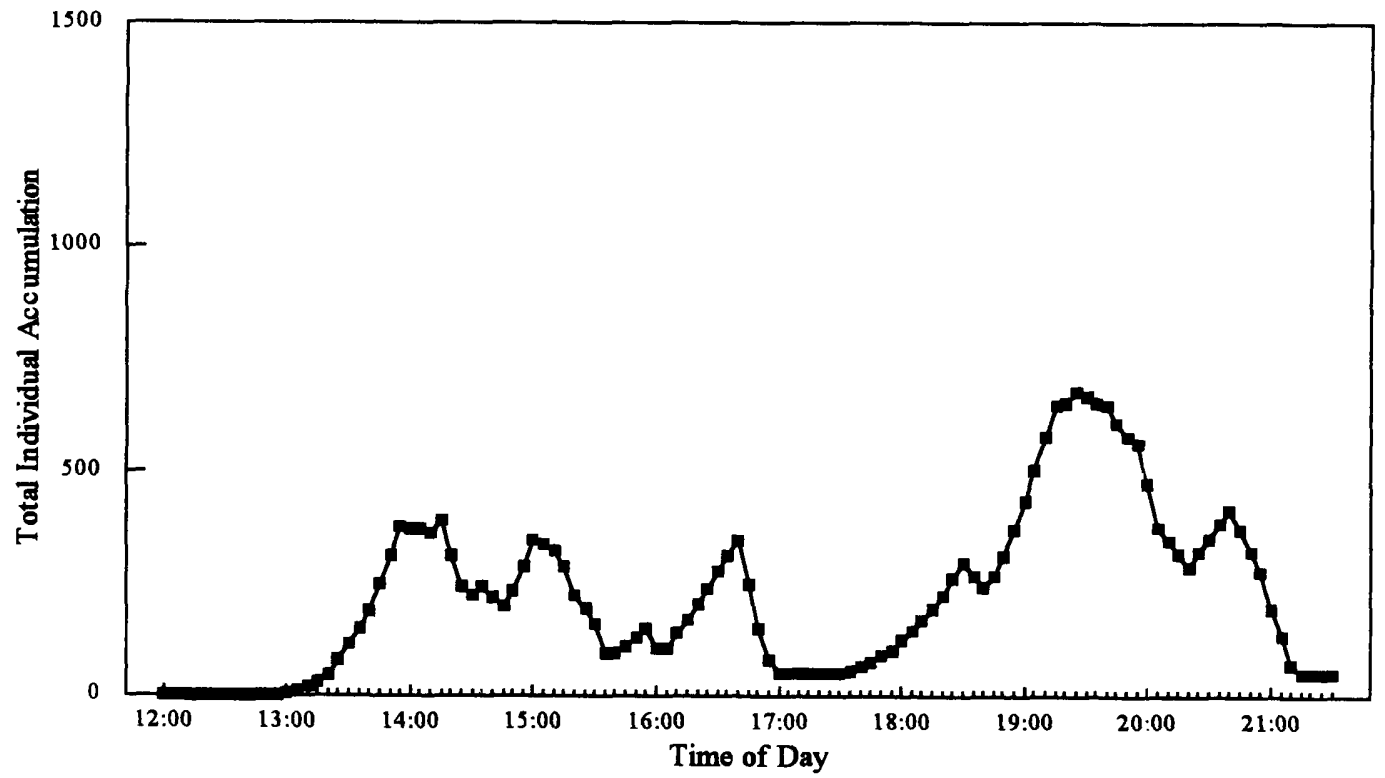

Figure 8.16: Total Individual Accumulation for Northwest Flights 
Note that the maximum accumulation, $Q^{*}(t)$, shown in Figure 8.12 is only 676 individuals, 42 percent lower than the 1,164 individuals for the "Other Airline" portion of the building. If we assume that an equivalent net waiting area exists for Northwest passengers and their well-wishers (i.e. 10,000 sq. ft), the available space afforded by Security-Check Plan \#3 translates into 1.37 square meters per individual, or level of service "B", as shown in Table 8.3. The FTA report concurs :

On the Northwest side, since narrow-body aircraft are used in some of the afternoon peak hour flights, there is still comfortable waiting space left. But this condition can change if higher capacity aircraft are introduced for those flights in the future. [p. 4]

Again, the fact that the demand is distributed over two peaks, one in the afternoon and one in the evening, reduces the maximum potential congestion (as measured by total individual accumulation) within the waiting area, despite the fact that a greater total number of individuals are expected to pass through the Northwest area than the "Other Airline" area.

The intent of this case study was to demonstrate, as proof of concept, that simple numerical techniques can be used to achieve results similar to those obtained by more detailed, Monte Carlo simulation programs when trying to estimate potential congestion. These simple techniques, however, cannot replace the usefulness of such detailed simulation programs for more in-depth analyses of passenger flows and service facility performance. Rather, the techniques we have demonstrated, similar to those introduced in previous chapters, are intended to facilitate "back-of-the-envelope" type analyses during the initial planning stages of the design of airport passenger buildings. Once inappropriate design alternatives have been eliminated using these techniques, detailed simulation programs can be applied to the smaller subset of alternatives to determine which is most appropriate based on more precise information. 


\title{
Chapter 9
}

\section{Conclusions and Suggestions for Future Research}

\author{
Introduction
}

The historical perspective of the airport as a terminal, or beginning or ending point only of a traveler's journey, must change. This perspective, still widely held in current practice, wrongly encourages planners to focus on a limited number of performance criteria related only to originating and terminating passenger traffic. The continued emergence of hub-and-spoke operations at today's airports means that consideration for transfer passengers is becoming correspondingly important. Good design practice dictates that quality or performance be considered using multiple criteria over a wide range of possible conditions. Such a philosophy encourages planners to view design strategy in terms of performance "robustness", i.e. in broad-range, multiple criteria terms. With this perspective, it is more appropriate to think of airports as a collection of "passenger buildings", rather than simply terminals.

This dissertation has identified the need for a set of fast and flexible decision support tools to aid airport planners in following good design practice when selecting an initial configuration of passenger buildings. We refer to these tools generally as Passenger Building Configuration Evaluator, or PBCE 
tools. The tools take advantage of inexpensive microprocessors to perform "back-of-the-envelope"-type calculations to assess the potential performance of different configuration types in order to eliminate inappropriate design alternatives.

Chapter 9 summarizes the findings of this thesis and suggests areas for further research. Section 9.1 discusses the inadequacies of current decision support methodologies related to the selection of an initial configuration. Section 9.2 reviews the general airport configuration taxonomy introduced in Chapter 3 and discusses how geometric characterizations of these concepts can be used to provide an objective platform for measuring the relative performance of different geometries. Section 9.3 reviews the overall modeling process followed to develop the PBCE tools, and describes how similarly structured data can be used to estimate potential configuration performance related to passengers, baggage, and aircraft. Section 9.4 summarizes the general observations drawn from numerical experiments performed on the configurations characterized previously. Finally, Section 9.5 discusses the areas of possible future research in the area of planning level decision support for the design of airport passenger buildings.

\section{1 - Current Decision Support is Inadequate}

The need for a fast and flexible decision support methodology stems from the shortcomings found in current methods, i.e. reference manuals and texts, analytic formulae techniques, and detailed Monte Carlo simulation programs. Each method provides some degree of either speed or flexibility, but none provides a high level of both.

\section{Reference Manuals and Texts}

Of the current methods, reference manuals and texts provide the fastest decision support for both the selection of an initial configuration and for the detailed floor layout (once an initial configuration has been selected); however, such tools tend to consider only a limited number of criteria with which to compare different configurations, and they cannot provide extensive sensitivity analyses over a wide range 
of conditions. For this reason, they are typically useful only for broad, descriptive overviews of different configurations. Such decision support, therefore, is unable to provide any sort of quantitative estimates for the potential performance of specifically defined configurations, making them highly inflexible for all but the most basic comparisons. The PBCE tools developed in this thesis provide a significant improvement over the "cookbook"-type approach of these reference manuals and texts, in that specific, very different configurations can be compared quantitatively over a wide range of user defined conditions.

\section{Analytic Formulae Techniques}

Unlike reference manuals and texts, the analytic formulae techniques described in previous chapters provide a greater degree of flexibility in the number and type of configuration concepts they are able to consider. By mathematically characterizing performance measures such as overall passenger walking distances, these techniques can be applied objectively to many different configuration types and geometries over a variety of different conditions such as the level and type of transfer traffic and industry structure. In order to obtain a single expression for a given performance measure, however, several simplifying assumptions must be made, including a uniform gate size and utilization and a uniform distribution of passengers within each individual concourse. Such assumptions severely limit the usefulness of results obtained from these techniques.

\section{Monte Carlo Simulation Programs}

Unlike the previous two approaches, detailed simulation programs provide a great deal of flexibility in the number of different configurations they can consider and the range of conditions over which they can study them. These programs represent the airport environment dynamically, typically "simulating" individual passenger movements in order to estimate different measures of performance. To model the movement of passengers accurately, these programs often require much detailed information regarding both the airport layout, and the intended schedule of daily flights. Acquiring such information 
is time consuming, however, and thus limits the usefulness of such programs for considering many different design alternatives (i.e. the selection of an initial configuration).

\section{Summary}

None of the previously mentioned techniques provide airport planners with a fast, highly flexible platform to aid in the decision making process. The PBCE tools developed in this dissertation, however, use simple mathematical expressions (based on well-known results of geometrical probability) in an object-oriented environment to estimate potential configuration performance. Moreover, the models used in the PBCE tools do not depend on any particular assumptions regarding gate utilizations or the distribution of passengers within the airport. Thus they can be used to model as simple or as complex a set of assumptions as is available to the user. Additionally, because the models can be implemented using even a very small amount of information, they can virtually eliminate the long setup times associated with the detailed Monte Carlo simulations.

\section{2 - Characterizing Different Airport Configurations}

This dissertation has extended the general airport taxonomy to include the midfield concept as one of the four primary types of airport configurations. These concepts are part of a progression or evolutionary process which began with the first airport buildings in the 1930's. As the volume and nature of airline passenger traffic changed, so did the configuration concepts used as the interface between passengers and aircraft. Although each individual airport is, in fact, unique, an overwhelming number of them can be placed into these four categories based on the geometry of the individual passenger buildings and the operational philosophy in place. 


\section{Review of Airport Configuration Concepts}

The gate arrival, or linear design is the oldest and simplest configuration concept. It provides the most direct access to aircraft for originating and terminating passengers, for whom walking distances are essentially reduced to the width of the building. As passenger volumes increased, there was a corresponding need for greater aircraft frontage, which helped bring about the second generation, or pier configuration concept. The introduction of piers brought about a new method of passenger processing; namely, maintaining separate holding facilities for passengers on different flights.

The third generation of passenger building configurations is represented by the satellite concept The motivation behind the satellite concept was the increased maneuverability afforded aircraft, particularly if satellites were connected to the terminal building with below-grade connectors. This feature allows aircraft to be parked around the entire perimeter of the satellite without having to negotiate through cul-de-sacs or dead-ends during entry and exit. Similar to satellites, the transporter or remotegate concept has the potential to reduce apron congestion by transporting passengers to aircraft, rather than vice-versa. This concept also reduces the need for fixed facilities beyond the main terminal block, which makes it convenient for dealing with seasonal and/or peak-hour traffic.

The most recent generation of configuration concepts is the midfield design. Again, similar to the satellite concept, individual passenger buildings are disjoint from the main terminal building, allowing for greater aircraft maneuverability and increased frontage along the concourse perimeter. Originating and terminating passengers often access the concourses through some sort of people moving device, whereas transfer passengers can generally remain "midfield".

Each of the preceding configuration concepts can be combined with one or more of the others to form "hybrid" designs. Hybrid configurations can be created either by design or by expansion, depending on the existing configuration. Hybrid-by-design airports have the potential advantage of borrowing the most desirable features from each of the "pure" designs to create a configuration that may be more flexible to changes in future demand patterns. 


\section{Characterizing Airport Configuration Concepts Geometrically}

In addition to the general descriptions provided previously, we characterized each of the different configuration concepts geometrically. Such characterizations allowed us to establish an objective platform from which to base quantitative comparisons. For example, in comparing a finger-pier configuration with a midfield configuration of the same size (as measured by the total number of gates), it is helpful to set constant such parameters as the gate spacing and the size of the terminal block, so that differences in estimated performance arise from the inherent geometry, rather than extraneous variables. In order to characterize all the different configuration concepts, we extended the work of previous authors using similar notation and constructions.

From these geometric characterizations, we determined the first set of critical inputs into most of our models for estimating potential performance; namely, the absolute distances between any two points at the airport. For measures of passenger convenience, we are concerned primarily with distances within the confines of the airport passenger buildings. For baggage and aircraft measures, however, we are also concerned with the distances outside the passenger buildings, such as the distances of paths traversed by ramp baggage trucks and by aircraft between exit taxiways and gates.

To estimate the potential performance of a given configuration (based on expected distances), we also need the probabilities that each of the distances obtained (from the geometric characterizations) will be traversed by "individuals" (i.e. passengers, baggage, or aircraft). These transition probabilities constitute the second set of critical inputs into our models, and are obtained from assumptions regarding the distribution of the individuals throughout the airport.

\section{3 - PBCE Tools are Designed in a General Framework}

The PBCE tools developed in this thesis are based on well-known results of geometrical probability. Specifically, they rely on the calculation of the moments of random variables, such as the mean and variance (or standard deviation). In order to obtain the expected value of a given performance 
measure (overall walking distances, for example), we need all the possible values the random variables can take (the distances of each path) and the likelihood each value will occur (the transition probabilities). The moments (as well as other supplementary statistics) can then be calculated using standard formulae.

\section{Object Orientation}

To estimate the necessary transition probabilities the PBCE tools were developed using an objectoriented problem solving structure. Under this general structure, the airport environment is decomposed into different "objects", each having a list of attributes which characterize that particular object within the environment. For example, one representation includes different objects for passenger buildings, gates, aircraft, and the individual passenger categories considered for analysis. Functions then operate on the specific attribute values of these objects in order to obtain estimates for the transition probabilities (based on the amount of information available to the user).

Given no information regarding the distribution of passengers, the PBCE tools can estimate potential configuration performance using "uniform" distribution assumptions, such as those employed in previous literature. The expected value or first moment is then simply the arithmetic mean of all the possible path distances. Such assumptions, however, are unlikely to model the "true" distribution of passengers within an airport. Thus, the PBCE tools can also estimate transition probabilities which try to incorporate the effects of different operational practices which take place at the airport

\section{Intelligent Scheduling}

Effective stand management policies on behalf of the airlines and/or airport operators may influence the distribution of direct transfer passengers departing from within their arrival concourse. If information regarding the stand management policies is available, the user can incorporate the potential effects of those policies into the estimation of the transition probabilities for direct transfer passengers. This dissertation presented a simple model in which the probability of a direct transfer passenger 
departing from a particular gate (within the arrival concourse) was inversely proportional to the distance to that gate from the arrival gate. Additional information regarding interterminal transitions (the likelihood that connecting passengers will depart from a different concourse) were incorporated into the overall transition probability estimates.

\section{Aircraft Effects}

In addition to effective stand management, the distribution of passengers is also influenced by the utilization of aircraft stands within the airport. Again, given no information, it may be reasonable to assume that the size and utilization of each gate in the airport is the same; however, many airports use gates preferentially, particularly for large aircraft. An uneven distribution of aircraft capacity gates can mean an uneven distribution of passengers, which, in turn, affects the probability of passengers arriving or departing from those gates. This dissertation demonstrated how such "aircraft effects" could be incorporated alone or into the previous estimates obtained when considering "intelligent scheduling".

\section{Dynamic Gate Selection}

In addition to effective stand management policies and aircraft effects, airlines and/or airport operators can take advantage of low demand periods by electing to operate only a subset of gates -effectively reducing the size of the airport and potentially improving performance (as measured by such metrics as passenger walking distances and baggage transfer times). Conversely, the operators can also elect to expand the capacity during high demand periods if remote aircraft parking is available. Such an action can potentially improve performance (as measured by potential passenger congestion within the buildings). This dissertation demonstrated how the judicious selection of gates during low demand periods could be used to exploit the daily variability of demand that exists at many airports. 


\section{No Restrictions on Complexity}

The preceding examples are provided to demonstrate the power of the object-oriented problem solving structure in trying to incorporate as much "real world" information into the performance estimates as possible. Given even more detailed information, the general, open-plan approach of the PBCE tools can be used to consider many other issues, including perhaps the effects of irregular operations, new security procedures, the introduction of larger capacity aircraft, and others. Because of the way data is maintained, the models used to estimate the chosen set of statistics essentially remain the same. Furthermore, other statistics can easily be added, since at all times the complete probability distribution of the performance metric is maintained. The next section details some of the conclusions we have drawn based on numerical calculations made using the PBCE tools developed in previous chapters.

\section{4 - Some General Observations}

As a proof of concept, we applied some of the PBCE tools developed in this dissertation to several different configurations of passenger buildings. The values for many of the input parameters were taken from the previous literature (for those configurations studied previously), and consistent parameter values were developed for those concepts studied for the first time. Based on the results of these numerical "tests", we are able to draw some general conclusions regarding the relative performance of the different concepts, as well as the sensitivity of the performance metrics to values of the input parameters. In order to draw these conclusions, we relied on various "performance profiles", a way of displaying numerical data that helps planners view performance in terms of robustness. 


\section{Centralized vs. Decentralized Configurations}

Perhaps the most fundamental conclusion we can draw based on the results of our numerical calculations is that there exists a difference in the general behavior of profile lines between centralized and decentralized configurations. For example, profile lines drawn to find the most robust geometry to changes in levels of transfer traffic, $P$, have a well-defined minimum (the number of concourses, $n^{*}$, that minimizes a particular performance metric, such as walking distances), which remains constant as we vary $P$ across the entire range of possible values (Figure 9.1).

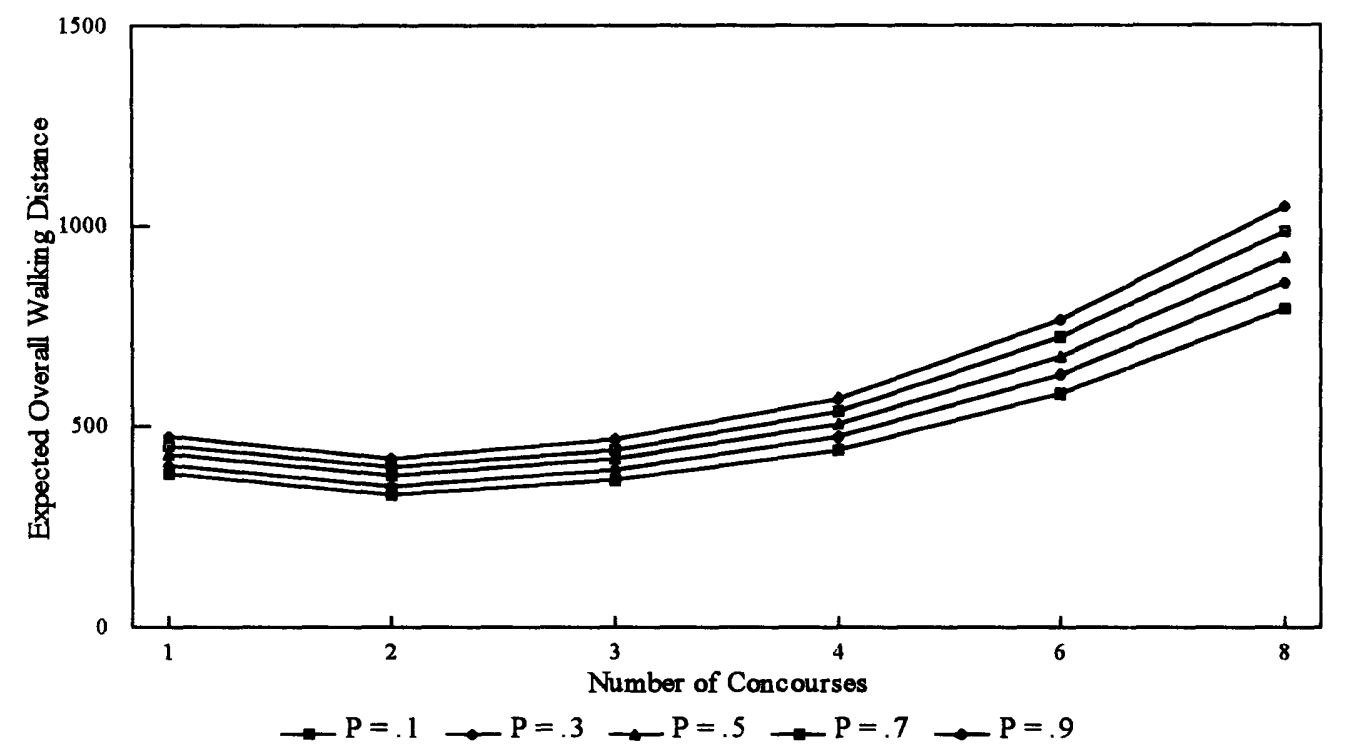

Figure 9.1: Characteristic Performance Profile for Centralized Configurations

This behavior suggests that, in general, the most appropriate geometry for centralized configurations is insensitive to the level of transfer traffic, $P$.

In contrast, the general behavior of profile lines for decentralized configurations is characterized by changing "minima" depending on the particular value of $P$ (Figure 9.2). Thus, there does not exist a single "optimal" $n$ " that minimizes the performance metric. This dependence on $P$ makes the selection of the most appropriate geometry non-unique and dependent on additional information. For example, we 
demonstrated that the selection process can be structured in a decision analytic framework, such that the additional information required is the confidence in our forecast for the value of $P$.

In the decision analytic framework, we expressed the "risk" involved with selecting a particular geometry as the maximum change in the performance estimate between the forecast and actual value of $P$ (given perfect information). We then calculated the required confidence level in our forecast, $\alpha$, (based on some utility function for performance) necessary to elicit selection of each of the candidate choices.

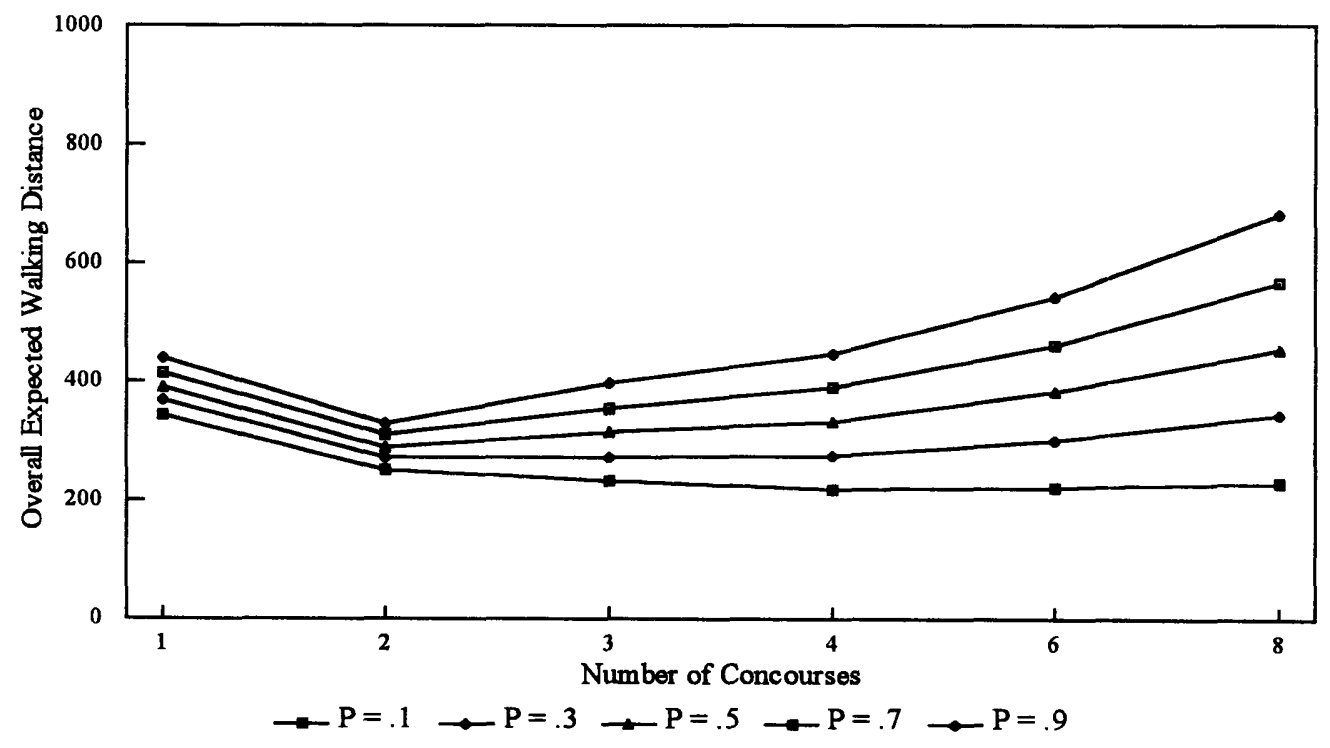

Figure 9.2: Characteristic Performance Profile for Decentralized Configurations

\section{"Best of the Best" Analyses}

Based on similar analyses for all the different configuration types considered, we then compared the most robust geometries across concept types in order to recommend the most appropriate configuration for a given set of circumstances (in this case, level and type of transfer traffic). Based on the specific set of input data used (taken from previous literature), we found that for a 24-gate airport expecting low levels of transfer traffic (i.e. a high level of originating and terminating traffic), the decentralized gate arrival design provided the most robust performance, as measured by overall expected walking distances. 
This conclusion, though perhaps intuitive, formalizes in a quantitative manner the belief held by many airport planners, including those responsible for the design of the Dallas/Ft. Worth International Airport (which is a decentralized gate arrival configuration). As mentioned previously, at the time the Dallas/Ft. Worth Airport was being designed, passenger traffic was indeed mostly origin-destination It was not until the deregulation of the U.S. interstate airline industry in 1978 that carriers began to establish "hub-and-spoke" type networks, such as American Airlines did in Dallas/Ft. Worth.

As the level of transfer traffic increases, we found the midfield " $\mathrm{X}$ " design becomes the most appropriate configuration (in terms of passenger walking distances only). The "X"-shaped concourse minimizes the expected distance transfer passengers will have to walk (among those configurations considered), and thus begins to outperform the decentralized gate arrival design as $P$ increases. Given a "greenfield" site and a forecast for the expected level of transfer traffic, $P$, we can thus use the previous results (in part) to suggest the most appropriate configuration type; however, it is important to consider as many performance metrics as possible (i.e. to consider performance in broad-range, multiple criteria terms).

\section{Considering Other Measures}

Our previous analysis of the most appropriate configuration type considered only one dimension of potential performance; namely, overall expected walking distances. Other performance metrics exist, however, which can also be studied using the PBCE tools. For example, this dissertation also considered the expected taxi distance and required number of turns for arriving aircraft. In a simple numerical example, we demonstrated that the "X"-shaped concourse, although it minimized the expected walking distance for passengers, actually increased both the expected taxi distance and the required number of aircraft turns over an equivalently sized linear concourse.

Thus, as we have suggested, the selection of the most appropriate configuration is a multidimensional decision. Similar to the selection of the most appropriate geometry for decentralized configurations, the overall selection of the initial configuration could be accomplished using a decision 
analytic framework, where the utility of each performance metric is specified, and confidence levels are established for each particular decision.

\section{Effects of Industry Structure}

In addition to robustness in terms of the level and type of transfer traffic, this dissertation also considered the robustness of different configurations to changes in industry structure, as measured by the number of "effective" competitors, $C$, operating at an airport. Previous authors have attempted to measure industry structure by defining a parameter $r$, which represents the fraction of direct transfer passengers who are certain to depart from their arrival concourse. The difficulty with this definition is that $r$ may not remain constant for different geometries of a given configuration.

Therefore, we presented a method for estimating the appropriate values of $r$ for individual geometries based on the number of individual concourses, $n$, and the number of competitors, $C$. Then, using an analysis similar to the one for testing robustness to changes in transfer traffic, we were able to test the robustness of different configurations to changes in industry structure. Specifically, for any ordered pair $(P, C)$ we showed we could find the most appropriate geometry and configuration based on some stated objective.

In general, we again found that for low levels of transfer traffic, the decentralized gate arrival configuration performs best of those concepts considered, due to the relatively short walking distances for originating and terminating passengers. As the level of transfer traffic increased, the most appropriate configuration type became sensitive to the number of effective competitors, $C$. For a small number of competitors, the centralized midfield " $\mathrm{X}$ " configuration provided the best overall performance, as it minimized the likelihood that transfer passengers would have to make an interterminal transition. As the number of competitors increased, however, it became more appropriate to have individual parallel pier concourses for each competitor. 


\section{Potential Congestion}

The final element of configuration performance we considered was that of potential passenger congestion, both in walkways and within waiting areas. In order to minimize congestion, one would ideally like to build an airport as large as possible. In order to minimize factors such as passenger walking distances and baggage transfer times, however, one would like the airport to be as small as possible. Thus, in studying factors that "push" the selection of the most appropriate geometry and configuration in opposite directions, we are able to strike a balance between the conflicting objectives of each.

This dissertation demonstrated that using PBCE tools involving simple, "spreadsheet"-type equations, it was possible to achieve congestion estimates that were consistent with other results obtained from detailed, Monte Carlo simulation programs (requiring as many as three weeks of data collection and setup time). We are not suggesting that the PBCE tools are an adequate substitute for more sophisticated decision support methodologies. We are suggesting, however, that some of the tasks traditionally carried out by these more sophisticated methods can now be accomplished more quickly using the PBCE tools presented in this dissertation.

\section{5 - Suggestions for Future Research}

The PBCE tools introduced in this dissertation provide a broad foundation upon which other tools can be developed for estimating the potential performance of different configurations of airport passenger buildings. The object-oriented, "open-plan" approach that serves as the basic structure of the PBCE tools can easily be expanded to consider other measures of potential performance, and measures that we have already introduced can be examined in greater depth. This section suggests the some of the possible extensions of this body of work, both in terms of modeling and implementation issues. 


\subsection{1 - Modeling Issues}

This section suggests some of the other modeling issues that could be incorporated into a broader set of PBCE tools in the future. They include suggestions for studying other performance measures, as well adding to the complexity of measures already considered.

\section{Different Performance Measures}

One natural extension of this thesis is to incorporate a wider range of performance measures into the general PBCE framework. As mentioned in Chapter 2, the topic of what constitutes a "good" airport is the subject of much research and debate. By incorporating issues that represent the objectives of all three primary "users" of an airport (i.e. the owners/operators, airlines, and passengers), planners can better assess the trade-offs involved with selecting alternative designs as they strive to achieve broadrange, multiple criteria performance goals.

On behalf of the owners, a generalized cost model might be developed to prevent the tools from suggesting "overdesign" when trying to reduce potential congestion. Additionally, issues related to the placement of security devices could be studied to see how they affect potential congestion and the overall travel times of both passengers and baggage. On behalf of the airlines, it may be possible to apply some of the "dynamic" tools used for estimating potential congestion to the modeling of passenger service facilities such as check-in and baggage claim areas.

Finally, on behalf of passengers, we might combine the overall travel time model with the potential congestion model to vary the average walking speed of pedestrians in crowded spaces. Such a model could then be used to estimate "true" connection times at hubs, as well as the minimum required time passengers must arrive at an airport before their scheduled flight departure. 


\section{Alternative Stand Management Models}

In addition to studying alternative measures of potential performance, research could be done in providing empirical evidence for the models already presented. For example, we argued that because of effective stand management policies practiced by the airlines and/or airport operators, the uniform distribution may not be the most appropriate model for determining transition probabilities (for direct transfer passengers who depart from their arrival concourse). One alternative we suggested was a triangular distribution, such that passengers were more likely to depart from gates closer to their arrival gate. Other certainly exist. By comparing actual flight data with operational records (detailing which flights were assigned to which gates) one might be able to develop an improved and perhaps more general model for studying the effects of "intelligent scheduling" on the distribution of passengers within an arrival concourse.

\section{Incorporating Irregular Operations into Stand Management Policies}

In addition to incorporating alternative models of different stand management policies, research could also be pursued to incorporate the effects of irregular operations on these policies. For example, flight delays due to "upline" cancellations and maintenance problems may increase gate occupancy times significantly. If the delayed aircraft occupies a gate generally used for "preferential" flights, the benefits of effective stand management may be diluted. Some policies may be more robust than others to the detrimental effects of such irregular operations. Based on the amount of information available to the user, different policies for assigning flights-to-gates could be tested using the PBCE tools. 


\section{Adding an Apron Congestion Model to Aircraft Taxi Model}

In Chapter 7 we presented a model for estimating the overall expected taxi distance and required number of turns for arriving aircraft. We suggested that based on a given relationship between taxi speed and the number of required turns, we could also estimate the overall expected taxi time for arriving aircraft (in the absence of any apron congestion). The effects of apron congestion, however, are nontrivial, and constitute a significant portion of the overall taxi time for arriving and departing aircraft. Therefore, any model to estimate overall aircraft taxi times should include some element of potential delay due to apron congestion.

Similar to passenger congestion, however, apron congestion is time-dependent, and relies on the actual flight schedule and gate assignments of aircraft. It may be difficult, then, to model this component of the estimate for taxi times in a planning context. As we have demonstrated in this dissertation, however, there may some "back-of-the-envelope"-type analysis that could capture at least some of the congestion effects, and help planners make better decisions in the absence of future flight schedules.

\subsection{2 - Implementation Issues : A Graphical Interface}

The most important implementation issue to address is the need for a graphical interface for the current set of PBCE tools. An effective graphical interface could be used for both input and output to the models we have presented in this dissertation. As an input device, a graphical interface would allow users to construct geometric representations of any type of configuration concept, including hybrid designs or concepts which deviate from those presented in Chapters 3 and 4. Several commercially available computer-aided design programs have the capability to determine distances between any two points on a given layout (and to scale appropriately). Thus it is possible that they could be used as a "front-end" to the mathematical models contained within the PBCE tools themselves.

As an output device, a graphical interface could be used to study many of the numerical data

which result from the PBCE models. We have already seen that data represented in the form of 
performance profiles can provide planners with trend information which would be more difficult to infer from numerical data alone. In addition, we have discussed different ways of presenting potential congestion estimates in the form of "congestion profiles". Finally, we could use the graphical capabilities of the computer-aided design programs mentioned previously to display the most appropriate configurations (and corresponding geometries) based on several different objectives of performance, as illustrated in Chapter 6.

The PBCE tools developed in this dissertation provide a fundamental platform from which many different applications can be extended. The use of a graphical interface would add visual impact to the results obtained from these models, and would help planners identify performance behavior under the uncertainty of different input parameters. More generally, however, the simple, flexible approach of the PBCE tools provides planners with important information quickly, which will ultimately help them make better decisions when selecting an initial configuration of passenger buildings. 


\section{Appendix A - Simulation Input Data}

Table A. 1 - Input Flight Schedule (Thursday, August 1988)

\begin{tabular}{|c|c|c|c|c|c|}
\hline Dept. Time & Carrier & Flt \# & Equip. & Dest. & \# Seats \\
\hline 1420 & NW & 49 & D10 & DTW & 284 \\
\hline 1430 & $\mathrm{AC}$ & 805 & D9S & YYZ & 116 \\
\hline 1440 & NW & 1647 & D10 & DTW & 284 \\
\hline 1440 & NW & 1651 & $72 S$ & $\mathrm{DCA}$ & 146 \\
\hline 1500 & NW & 41 & 757 & ORD & 184 \\
\hline 1525 & NW & 35 & D10 & JFK & 284 \\
\hline 1545 & NW & 47 & 747 & MSP & 400 \\
\hline 1610 & $\mathrm{AC}$ & 672 & D9S & YHZ & 116 \\
\hline 1620 & NW & 1649 & D9S & $\mathrm{MKE}$ & 100 \\
\hline 1650 & $\mathrm{CP}$ & 406 & DH8 & YSJ & 37 \\
\hline 1705 & NW & 809 & M80 & MEM & 143 \\
\hline 1705 & NW & 389 & D10 & DTW & 284 \\
\hline 1705 & $\mathrm{AC}$ & 807 & $72 \mathrm{~S}$ & YYZ & 158 \\
\hline 1800 & LH & 423 & D10 & FRA & 282 \\
\hline 1855 & NW & 357 & 757 & MSP & 184 \\
\hline 1910 & $7 \mathrm{~F}$ & 921 & HS7 & YOW & 47 \\
\hline 1935 & LH & 456 & $\mathrm{D} 10$ & LAX & 282 \\
\hline 1940 & NW & 391 & $72 S$ & DTW & 146 \\
\hline 2000 & NW & 36 & D10 & $\mathrm{CPH}$ & 284 \\
\hline 2015 & NW & 46 & 747 & FRA & 400 \\
\hline 2030 & NW & 48 & 747 & LGW & 400 \\
\hline 2100 & $\mathrm{BA}$ & 214 & 747 & LHR & 419 \\
\hline 2100 & $\mathrm{EI}$ & 116 & 747 & SNN & 432 \\
\hline 2110 & $\mathrm{AF}$ & 12 & $74 \mathrm{M}$ & CDG & 261 \\
\hline 2110 & SR & 127 & 743 & ZRH & 384 \\
\hline 2115 & $\mathrm{AC}$ & 1898 & DH8 & YQI & 37 \\
\hline 2115 & NW & 34 & D10 & PIK & 284 \\
\hline 2130 & NW & 40 & $\mathrm{D} 10$ & SNN & 284 \\
\hline
\end{tabular}

Table A.2: Average Load Factor

$\mathrm{ALF}=80 \%$ 
Table A.3 : Simulated Arrival Distributions of Originating Passengers

\begin{tabular}{crcr}
\multicolumn{2}{c}{ International } & \multicolumn{3}{c}{ Domestic } \\
Time before flight ${ }^{1}$ & Cumulative \% & Time before flight ${ }^{1}$ & Cumulative \% \\
\hline & & & \\
$95-150$ min. & 11.5 & $75-85$ min. & 2.0 \\
$65-95$ & 38.0 & $55-75$ & 16.0 \\
$45-65$ & 68.0 & $40-55$ & 42.5 \\
$25-45$ & 90.0 & $15-40$ & 93.0 \\
$10-25$ & 100.0 & $10-15$ & 100.0 \\
\hline
\end{tabular}

Table A.4: Well-Wishers per Passenger

\begin{tabular}{cr} 
\# of Well-Wishers & Cumulative \% \\
\hline 0 & 85.00 \\
1 & 91.23 \\
2 & 96.23 \\
3 & 97.57 \\
4 & 98.29 \\
5 & 99.01 \\
6 & 100.00 \\
\hline
\end{tabular}

Table A.5 : Originating versus Transferring Passengers

\begin{tabular}{lrr} 
& Northwest & Other Airlines \\
\hline & & \\
Originating & $80 \%$ & $90 \%$ \\
Terminating & $20 \%$ & $10 \%$ \\
\hline
\end{tabular}

\footnotetext{
${ }^{1}$ Note: Rounded to the nearest 5-minute interval.
} 


\section{Bibliography}

Anglo Japanese Airport Consortium (1992) KL International Airport Master Plan for the Government of Malaysia, Volume B: Airport Master Plan, December.

Ashford, N., Stanton, H.P., and Moore, C.A. (1991) Airport Operations, John Wiley \& Sons, New York, NY.

Ashford, N., and Wright, P.H. (1979) Airport Engineering, John Wiley \& Sons, New York, NY.

Babic, O., Teodorovic, D., and Tosic, V. (1984) "Aircraft Stand Assignment to Minimize Walking Distances," Transportation Engineering Journal of ASCE, Vol. 110, No. 1, January, pp 55-66.

Bandara, S. (1990) "Optimum Geometries for Satellite-Type Airport Terminals," Transportation and Traffic Theory, M. Koshi, ed., Elsevier Science Publishing Co., Inc., New York, NY, pp. 409-428.

Bandara, S. (1989) "Airport Terminals -- Optimum Configurations and Gate Position Requirement," Ph.D. Dissertation, Department of Civil Engineering, The University of Calgary, Calgary, Alberta, Canada.

Bandara, S., and Wirasinghe, S.C. (1992a) "Optimum Geometries for Pier-Type Airport Terminals," Journal of Transportation Engineering, Vol. 118, No. 2, March/April, pp. 187-206.

Bandara, S., and Wirasinghe, S.C. (1992b) "Walking Distance Minimization for Airport Terminal Configurations," Transportation Research A., Vol. 26A, No. 1, January, pp. 59-74.

Bandara, S., and Wirasinghe, S.C. (1989) "Airport Gate Position Estimation Under Uncertainty," in Transportation Research Record 1199, Transportation Research Board, National Research Council, Washington, DC, pp. 41-48.

Best, Alastair, (1991) "Taking flight," The Architectural Review, Vol. CLXXXIX, No. 1131, May, pp. 58-61.

Birch, C. (1992) "The best laid plans ...," Airport Review, Vol. 4, No. 9. p. 3.

Blankenship, E.G. (1974) The Airport; Architecture, Urban Integration, Ecological Problems, Praeger, New York, NY.

Braaksma, J.P. (1977) "Reducing Walking Distances at Existing Airports," Airport Forum, Vol. 4, August, pp. 135-142.

Braaksma, J.P., and Shortreed, J.H. (1975) "Method for Designing Airport Terminal Concepts," Transportation Engineering Journal of ASCE, Vol. 101, No. TE2, May, pp. 321-336.

Byrne, D.M., and Taguchi, S. (1987) "The Taguchi Approach to Parameter Design," Quality Progress, December.

Davey, P. (1991) "Airports come of age", The Architectural Review, Vol. CLXXXIX, No. 1131, May, pp. 35-45.

de Neufville, R. (1994) "Designing Airport Passenger Buildings for the 21st Century," accepted for Transport Journal, Proceeding of the Institution of Civil Engineers (UK), Paper 10284. 
de Neufville, R. (1990) "Successful Siting of Airports: Sydney Example", Journal of Transportation Engineering, Vol. 116, No. 1, January, pp. 37-48.

de Neufville, R. (1976) Airport Systems Planning: A Critical Look at the Methods and the Experience, The MIT Press, Cambridge, MA, (and in the United Kingdom), The Macmillan Press, London.

de Neufville, R., and Grillot, M. (1982) "Design of Pedestrian Space in Airport Terminals," Transportation Engineering Journal Of ASCE, Vol. 108, No. TE1, January, pp. 87-101.

de Neufville, R., and Rusconi-Clerici, J. (1978) "Designing Airport Terminals for Transfer Passengers," Transportation Engineering Journal of ASCE, Vol. 104, No. TE6, November 1978, pp. 775-787.

Domestic Origin-Destination Survey of Airline Passenger Traffic, Civil Aeronautics Board, U.S. Government Printing Office, Washington, DC, Semiannual.

Dunlay, W., and McCullough, F. et al. (1975) "A Systems Analysis Procedure for Estimating the Capacity of an Airport: System Definition, Capacity Definitions and Review of Available Models," Council for Advanced Transportation Studies, University of Texas at Austin, October.

The Economist (1991) "Trees? Islands?", The Economist, Vol. 318, No. 7699, March 23, 1991, p.98.

Fay, D. R. (1971) "An Evaluation of Alternative Terminal Design for Airports," M.Sc. Thesis, Department of Civil Engineering, Massachusetts Institute of Technology.

Flight Transportation Associates (1990) "Logan Landside Demand/Capacity Analysis: Simulation Analysis of International Passenger Terminal E," Report FTA-TM-484-1, May, Cambridge, MA.

Fruin, J.J. (1971) Pedestrian Planning and Design, Metropolitan Association of Urban Designers and Environmental Planners, New York, NY.

Horonjeff, R., (1969) "Analysis of Passenger and Baggage Flows in Airport Terminal Buildings,", Aircraft Journal, Vol. 6, No. 5, pp. 446-451.

Horonjeff, R., (1962) Planning and Design of Airports, McGraw Hill, New York, NY.

Horonjeff, R., and McKelvey, F.X. (1983) Planning \& Design of Airports, McGraw Hill , New York, NY.

Horonjeff, R., et al. (1959) "A Mathematical Model for Locating Exit Taxiways," Institute of Transportation and Traffic Engineering, University of California, Berkeley, CA.

International Air Transport Association (1989) Airport Terminals Reference Manual, 7th Edition, Montreal.

International Air Transport Association (1981) Guidelines for Airport Capacity/Demand Management, Montreal, October.

Jahn, Helmut (1991) Airports, Birkhaüser, Basel.

Knowler, A.E. (1964) "The Use of the Erlang Loss Formula for the Calculation of the Required Number of Operation Stands," Proceedings from the 4th AGIFORS Symposium.

Koukoutsi, V.E. and Strabala, J.M. (1992) Arrival in Arabia : A New Terminal for the King Abdulaziz International Airport, Jeddah, The Kingdom of Saudi Arabia, Fall 1988 National Student Design Competition, American Institute of Architecture Students. 
Kumarage, A.S., and Wirasinghe, S.C. (1990) "Multinomial Mode Choice Models for Atlanta Airport Passenger Movement," Journal of Advanced Transportation, Vol. 24, No. 2, pp. 87-98.

Lemer, A.C. (1990) Characterizing and Measuring Performance for Airport Passenger Terminals. Report DTRS57-90-P-80851. Transportation Systems Center, U.S. Department of Transportation, July.

Mangoubi, R.S., and Mathaisel, D.F.X. (1985) "Optimizing Gate Assignments at Airport Terminals," Transportation Science, Vol. 19, No. 2, May, pp. 173-187.

Massport (1988) Airport Comparisons Study, The Planning and Programming Department, Massachusetts Port Authority, Boston, MA, June.

McKenzie, A.J., Hugget, J.W., and Ogden, K.W. (1974) "Staging of Improvements to Air Transport Terminals," Transportation Journal of ASCE, Vol. 100, No. TE4, pp. 855-872.

Maldonado, J. (1990) "Strategic Planning -- An Approach to Improving Airport Planning Under Uncertainty," M.Sc. Thesis, Department of Civil Engineering, Massachusetts Institute of Technology.

Momberger, M., (1993a) "O'Hare Opens Largest International Terminal in USA," Airport Forum, Vol. XXIII, No. 4, August, pp. 10-12.

Momberger, M., (1993b) "CLK Work Starts in Earnest," Airport Forum, Vol. XXIII, No. 3, June, pp. $10-12$.

Mumayiz, S.A., (1990) "An Overview of Airport Terminal Simulation Models", presentation to the 69th Annual Meeting of Transportation Research Board.

Newell, G.F. (1971) Applications of Queueing Theory, Chapman and Hall, London

Odoni, A.R., and de Neufville, R. (1992) "Passenger Terminal Design," Transportation Research A, Vol. 26A, No. 1, January, pp. 27-35.

Pararas, J. (1977) "Analytic Models for the Design of Aircraft Terminal Buildings," M.Sc. Thesis, Department of Civil Engineering, Massachusetts Institute of Technology.

Paullin, R.C., and Horonjeff, R. (1969) "Sizing of Departure Lounges in Airport Buildings", ASCE Transportation Engineering Journal, May, pp. 267-277.

Rallis, T. (1967) Capacity of Transport Centres, Report 35, Copenhagen, Technical University of Denmark.

Robusté, F. (1991a) "Centralized Hub Terminal Geometric Concepts; I: Walking Distance," Journal of Transportation Engineering, Vol. 117, No. 2, March/April, pp. 143-158.

Robusté, F. (1991b) "Centralized Hub Terminal Geometric Concepts; II: Baggage and Extensions," Journal of Transportation Engineering, Vol. 117, No. 2, March/April, pp. 159-177.

Steuart, G.N. (1974) "Gate Position Requirements at Metropolitan Airports," Transportation Science, Vol. 8, No. 2, May, pp. 169-189.

Taguchi, G., and Clausing, D., (1990) "Robust Quality," Harvard Business Review, No. 1, pp. 65-75.

Tosic, V., (1992) "A Review of Airport Passenger Terminal Operations Analysis Modelling," Transportation Research A, Vol. 26A, No. 1, January, pp. 3-26. 
Transportation Research Board (1987) "Measuring Airport Landside Capacity," Special Report 215, Committee for the Airport Capacity Study, National Research Council, Washington, DC.

United States, Department of Transportation and the Ralph M. Parsons Company, (1975) The Apron and Terminal Building Planning Manual, Report \# FAA-RD-75-191

United States, Federal Aviation Administration, (1982) Airport Design Standards -- Transport Airports, Report \# AC 150/5300-12, August.

United States, Federal Aviation Administration, (1980) Airport Design Standards -- Airports Served by Air Carriers, Taxiways, Report \# AC 150/5335-1A, May.

United States, Federal Aviation Administration, (1971) Advisory Circular on Airport Master Plans, Report \# AC 150/5070-6.

United States, Federal Aviation Administration, (1969) Aviation Demand and Airport Facility Requirement Forecasts for Medium Air Transportation Hubs Through 1980, January.

Wilbur Smith Associates (1988) "Long-Range On-Airport Access Plans," Airside Mobility Study for the New Denver International Airport, June.

Wirasinghe, S.C. (1988) "Approximate Continuous Modelling of Passenger Walking Distance," in TIMS Proceedings, EURO IX TIMS XXVIII, July.

Wirasinghe, S.C., and Bandara, S. (1992) "Planning of Parallel Pier Airport Terminals with Automated People Moving Systems under Constrained Conditions," Transportation Research Record 1373, Transportation Research Board, National Research Council, Washington, DC, pp. 35-45.

Wirasinghe, S.C., and Bandara, S. (1990) "Airport Gate Position Estimation for Minimum Total Costs -Approximate Closed Form Solution," Transportation Research B., Vol. 24B, No. 4, August, pp. 287-297.

Wirasinghe, S.C., Bandara, S., and Vandebona, U. (1987) "Airport Terminal Geometries for Minimal Walking Distances," Transportation and Traffic Theory, N.H. Gartner and H.M. Wilson, eds., Elsevier Science Publishing Co., Inc., New York, NY, pp. 483-502.

Wirasinghe, S.C., and Perera, M. (1992) "Improved Customs Inspection at a Small International Airport," Journal of Advanced Transportation, Vol. 26, No. 2, pp. 185-199.

Wirasinghe, S.C., and Shehata M. (1988) "Departure Lounge Sizing and Optimal Seating Capacity for a Given Aircraft/Flight Mix - (i) Single Gate. (ii) Several Gates," Transportation Planning and Technology, Vol. 13-2, pp. 57-71.

Wirasinghe, S.C., and Vandebona, U. (1987) "Passenger Walking Distance Distribution in Single- and Dual-Concourse Centralized Airport Terminals," in Transportation Research Record 1147, Transportation Research Board, National Research Council, Washington, DC, pp. 40-45. 